\title{
Copper-Catalyzed Asymmetric Reductive Allylation of Ketones with 1,3-Dienes
}

Bin Fu, ${ }^{\dagger}$ Xiuping Yuan, ${ }^{\dagger}$ Yanfei Li, ${ }^{\dagger}$ Ying Wang,,${ }^{\dagger}$ Qian Zhang, ${ }^{\dagger}$ Tao Xiong, ${ }^{* \dagger}$ and Qian Zhang ${ }^{\dagger \ddagger}$

$\dagger$ Jilin Province Key Laboratory of Organic Functional Molecular Design \& Synthesis, Department of Chemistry, NortheastNormal University, Changchun 130024, China

¥State Key Laboratory of Organometallic Chemistry, Shanghai Institute of Organic Chemistry, Chinese Academy of Sciences, 345 Lingling Lu, Shanghai 200032, China

E-mail: xiongt626@nenu.edu.cn

Table of Contents

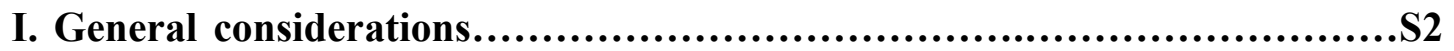

II. Experimental Details and Characterization..............................S3

III. Gram-scale synthesis and applications of chiral homoallylic tertiary

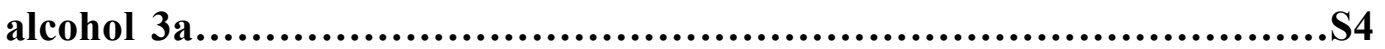

IV. Analytical data of new compounds..........................................S6

V. ${ }^{1} \mathrm{H},{ }^{13} \mathrm{C}$ and ${ }^{19} \mathrm{~F}$ NNR Spectra of new compounds........................... S36 


\section{General considerations}

General. All reactions were performed under nitrogen atmosphere in flame dried flasks. All reactions were monitored by thin layer chromatography (TLC) using Macherey-Nagel $0.20 \mathrm{~mm}$ silica gel 60 plates. Flash column chromatography was performed on silica gel 60 (particle size 300-400 mesh ASTM, purchased from Taizhou, China). ${ }^{1} \mathrm{H}$ and ${ }^{13} \mathrm{C}$ nuclear magnetic resonance (NMR) spectra were recorded on Bruker AV- 600 NMR spectrometers. ${ }^{1} \mathrm{H}$ and ${ }^{13} \mathrm{C}$ NMR spectra are reported in parts per million (ppm) downfield from an internal standard, tetramethylsilane $\left(0 \mathrm{ppm}\right.$ for $\left.{ }^{1} \mathrm{H}\right)$ and $\mathrm{CHCl}_{3}\left(77.0 \mathrm{ppm}\right.$ for $\left.{ }^{13} \mathrm{C}\right)$, respectively. High-resolution mass spectra (HRMS) were recorded on Bruck microtof. High-pressure liquid chromatography (HPLC) was performed on Agilent 1260 Series chromatographs using a chiral column $(25 \mathrm{~cm})$ as noted for each compound. Enantiomeric excess was determined by HPLC analysis employing Darcel Chiracel IA, IB and IC column. Optical rotation was measured on a Perkin-Elmer 341 polarimeter using a sodium lamp $(589 \mathrm{~nm})$ as the light source over a path length of $10 \mathrm{~cm}$.

Materials. Unless otherwise noted, commercial reagents were purchased from Energy-Chemical Limited, Alfa Aesar, and other commercial suppliers and were used as received. Diene substrates were synthesized according to procedures described in the literature. ${ }^{1}$ THF, toulene, $\mathrm{CyH}$ and $\mathrm{Et}_{2} \mathrm{O}$ were distilled over sodium and stored under nitrogen atmosphere.

1. Fiorito, D.; Folliet, S.; Liu, Y.; Maze, C. ACS Catal. 2018, 8, 1392-1398. 


\section{Experimental Details and Characterization}

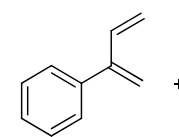

$1 \mathrm{a}$

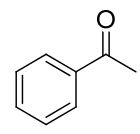

$2 a$

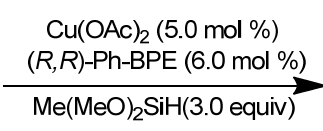

THF, $20^{\circ} \mathrm{C}, 18 \mathrm{~h}$

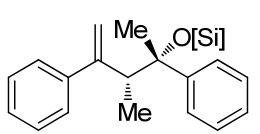

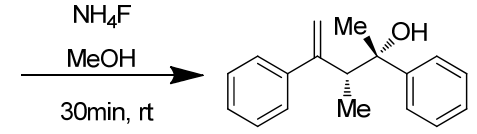

3a

Taking 3a as an example: In a nitrogen-filled glovebox, a flame-dried screw-cap reaction tube equipped with a Teflon-coated magnetic stir bar were charged with $\mathrm{Cu}(\mathrm{OAc})_{2}(1.8 \mathrm{mg}, 10.0 \mu \mathrm{mol})$, $(R, R)$-Ph-BPE $(6.1 \mathrm{mg}, 12.0 \mu \mathrm{mol})$, and anhydrous THF $(1 \mathrm{~mL})$, dimethoxymethylsilane (DMMS, 3 equiv) were subsequently added. The resulting mixture was stirred for $2 \mathrm{~min}$ and then buta-1,3-dien-2ylbenzene ( $52.0 \mathrm{mg}, 0.4 \mathrm{mmol})$ and acetophenone $(24.0 \mathrm{mg}, 0.2 \mathrm{mmol})$ were added, respectively. The vial was removed from the glove box, and the mixture was stirred at $20{ }^{\circ} \mathrm{C}$ for $18 \mathrm{~h}$. And then the cap was removed. The solution was quenched by adding saturated ammonium fluoride in methanol using a $5 \mathrm{~mL}$ syringe $(3 \mathrm{~mL})$ and stirred for $30 \mathrm{~min}$. After removal of solvent under reduced pressure with aid of a rotary evaporator, the diastereomeric ratio $(\mathrm{dr})$ was assessed by ${ }^{1} \mathrm{H} \mathrm{NMR}$ in $\mathrm{CDCl}_{3}$. The resulting crude product was purified by flash column chromatography on silica gel (diethyl ether /petroleum ether, 1:10) to obtain product. The details and characterization data of the products are stated below. It is noteworthy that the absolute configuration of the product 3a was determined by comparison of the HPLC spectra and optical rotation of $\mathbf{3}$ aj with a reported value. ${ }^{2}$ 


\section{Gram-Scale Synthesis and Applications of Chiral Homoallylic Tertiary Alcohol 3a}

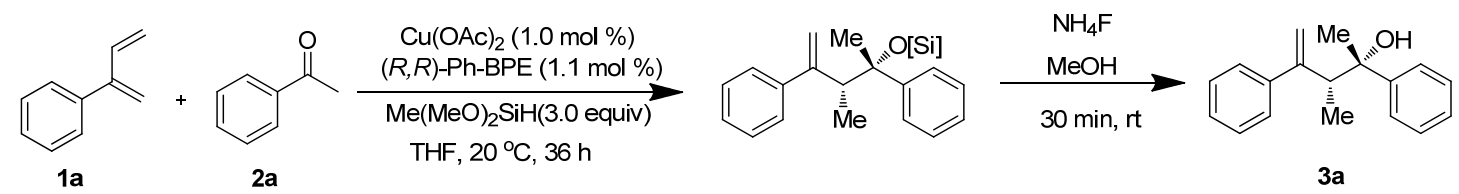

In an nitrogen-filled glovebox, $\mathrm{Cu}(\mathrm{OAc})_{2}(15.0 \mathrm{mg}, 1 \mathrm{~mol} \%),(R, R)-\mathrm{Ph}-\mathrm{BPE}(46 \mathrm{mg}, 1.1 \mathrm{~mol} \%)$, THF $(20 \mathrm{~mL})$ and dimethoxymethylsilane (DMMS, 3 equiv) were successively added into a $50 \mathrm{~mL}$ screwcapped vial containing a magnetic stirring bar. The resulting mixture was stirred for 2 min and then adding buta-1,3-dien-2-ylbenzene (1.62 g, 1.5 equiv) and acetophenone (1.00 g, $8.3 \mathrm{mmol}$ ), were added, respectively. The vial was removed from the glove box, and the mixture was stirred at $20{ }^{\circ} \mathrm{C}$ for $36 \mathrm{~h}$. After $36 \mathrm{~h}$ the cap was removed. The solution was quenched by adding saturated ammonium fluoride in methanol using a $5 \mathrm{~mL}$ syringe $(4 \times 5 \mathrm{~mL})$ and stirred for $30 \mathrm{~min}$. The resulting crude product was purified by flash column chromatography on silica gel (diethyl ether /petroleum ether, 1:10) to obtain product 3a (1.67 g, 80\%, 93\% ee).

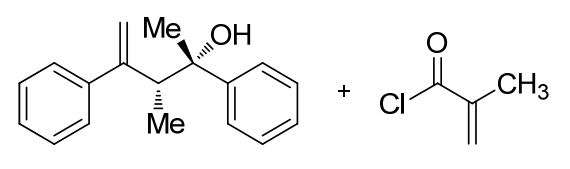

$3 \mathbf{a}$

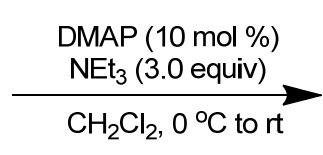

$\mathrm{CH}_{2} \mathrm{Cl}_{2}, \mathrm{O}^{\circ} \mathrm{C}$ to $\mathrm{rt}$

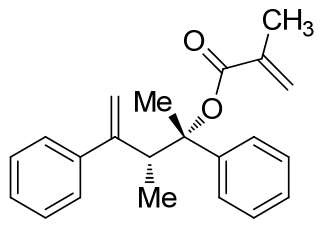

To a solution of (2S,3R)-3-methyl-2,4-diphenylpent-4-en-2-ol 3a (50.4 mg, 0.2 mmol, 1 equiv) in $\mathrm{CH}_{2} \mathrm{Cl}_{2}$ ( $1 \mathrm{~mL}$ ) was added DMAP (2.2 $\mathrm{mg}, 10 \mathrm{~mol} \%), \mathrm{Et}_{3} \mathrm{~N}(83 \mu \mathrm{L}, 3$ equiv), followed by methacryloyl chloride ( $20 \mu \mathrm{L}, 2$ equiv) at $0{ }^{\circ} \mathrm{C}$. The reaction mixture was warmed to room temperature over night, the reaction was quenched with aq. $\mathrm{HCl}(3 \mathrm{~mL}, 1 \mathrm{M})$. The aqueous layer was extracted with $\mathrm{CH}_{2} \mathrm{Cl}_{2}(3 \times 5 \mathrm{~mL})$. The combined organic layer was dried over $\mathrm{MgSO}_{4}$ and concentrated in vacuo and purified by flash chromatography over silica gel (EtOAc / hexane: 5 : 95) to afford (2S,3R)-3-methyl-2,4-diphenylpent-4en-2-yl methacrylate $4(28.5 \mathrm{mg}, 0.09 \mathrm{mmol}, 44 \%)$ as a colorless oil. ${ }^{1} \mathbf{H} \mathbf{~ N M R}\left(600 \mathrm{MHz}, \mathrm{CDCl}_{3}\right) \delta$ $7.22-7.15(\mathrm{~m}, 7 \mathrm{H}), 7.13-7.10(\mathrm{~m}, 3 \mathrm{H}), 5.90(\mathrm{~s}, 1 \mathrm{H}), 5.47(\mathrm{~s}, 1 \mathrm{H}), 5.29(\mathrm{~s}, 1 \mathrm{H}), 5.11(\mathrm{~s}, 1 \mathrm{H}), 3.24(\mathrm{q}, J$ $=7.2 \mathrm{~Hz}, 1 \mathrm{H}), 1.95(\mathrm{~s}, 3 \mathrm{H}), 1.87(\mathrm{~s}, 3 \mathrm{H}), 1.30(\mathrm{~d}, J=7.2 \mathrm{~Hz}, 3 \mathrm{H}) \cdot{ }^{13} \mathbf{C} \mathbf{N M R}\left(150 \mathrm{MHz}, \mathrm{CDCl}_{3}\right) \delta 165.8$, $150.5,144.7,142.8,137.5,128.0,127.5,126.71,126.68,126.4,125.5,124.9,116.5,86.4,50.1,22.5$, 18.4, 16.9. HRMS (ESI-TOF) $(\mathrm{m} / \mathrm{z})$ : Calcd for: $\mathrm{C}_{22} \mathrm{H}_{24} \mathrm{O}_{2} \mathrm{Na}: 343.1674$; found: 343.1665 .<smiles>C[C@@](O)(c1ccccc1)[C@H]1C=CCCC1</smiles>

3aj

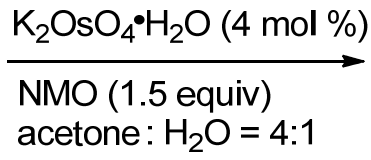
acetone: $\mathrm{H}_{2} \mathrm{O}=4: 1$

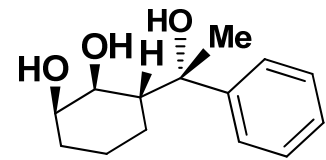

5

(1R,2S,3R)-3-((S)-1-hydroxy-1-phenylethyl)cyclohexane-1,2-diol 5 was synthesized according to the procedure described in the literature. ${ }^{3}$ To a solution of $(S)-1-((R)$-cyclohex-2-en-1-yl)-1-phenylethanol 3aj (40.5 mg, $0.2 \mathrm{mmol}, 1$ equiv) in acetone $(0.3 \mathrm{~mL})$ and water $(0.075 \mathrm{~mL})$ was added NMO $(0.035 \mathrm{~g}$, 
$0.3 \mathrm{mmol}, 1.5$ equiv) followed by potassium osmate(VI) dihydrate ( $2.9 \mathrm{mg}, 4 \mathrm{~mol} \%$ ). The resulting brown suspension was left to stir at room temperature overnight. The resulting mixture was quenched by addition of sat. aq. $\mathrm{Na}_{2} \mathrm{~S}_{2} \mathrm{O}_{3}(5 \mathrm{~mL})$ and extracted with EtOAc $(3 \times 5 \mathrm{~mL})$. The combined organic layer was dried over $\mathrm{MgSO}_{4}$ and evaporated in vacuo to give a yellow oil. The yellow oil was purified by flash chromatography $\quad\left(0-10 \% \quad \mathrm{MeOH}\right.$ in $\left.\mathrm{CH}_{2} \mathrm{Cl}_{2}\right)$ to give (1R,2S,3R)-3-((S)-1-hydroxy-1phenylethyl)cyclohexane-1,2-diol $5(37.7 \mathrm{mg}, 80 \%)$ as a yellow oil. ${ }^{1} \mathbf{H} \mathbf{~ N M R}\left(600 \mathrm{MHz}, \mathrm{CDCl}_{3}\right) \delta 7.42$ $(\mathrm{d}, J=7.2 \mathrm{~Hz}, 2 \mathrm{H}), 7.33$ (t, $J=7.2 \mathrm{~Hz}, 2 \mathrm{H}), 7.28-7.25(\mathrm{~m}, 1 \mathrm{H}), 5.31(\mathrm{~s}, 1 \mathrm{H}), 4.66(\mathrm{~s}, 1 \mathrm{H}), 3.75(\mathrm{~d}, J=$ $1.6 \mathrm{~Hz}, 1 \mathrm{H}), 3.12-3.10(\mathrm{~m}, 1 \mathrm{H}), 3.00(\mathrm{~s}, 1 \mathrm{H}), 2.12-2.08(\mathrm{~m}, 1 \mathrm{H}), 1.81-1.77(\mathrm{~m}, 2 \mathrm{H}), 1.63-1.60(\mathrm{~m}$, 4H), $1.38-1.36(\mathrm{~m}, 1 \mathrm{H}), 1.11-1.07(\mathrm{~m}, 1 \mathrm{H}), 0.71-0.64(\mathrm{~m}, 1 \mathrm{H}) .{ }^{13} \mathbf{C ~ N M R}\left(150 \mathrm{MHz}, \mathrm{CDCl}_{3}\right) \delta$ 143.7, 127.7, 126.9, 126.3, 79.1, 74.1, 69.5, 47.2, 30.1, 28.6, 26.5, 19.2. HRMS (ESI-TOF) (m/z): Calcd for: $\mathrm{C}_{13} \mathrm{H}_{20} \mathrm{O}_{3} \mathrm{Na}$ : 259.1314; found: 259.1317 .

According to the NOE experiment, we observed a signal between $\mathrm{H}_{\mathrm{a}}$ and $\mathrm{H}_{\mathrm{b}}$, which means the cis configuration of $\mathrm{H}_{\mathrm{a}}$ and $\mathrm{H}_{\mathrm{b}}$. In addition, no signal was observed between $\mathrm{H}_{\mathrm{b}}$ and $\mathrm{H}_{c}$ and hence the trans configuration between $\mathrm{H}_{\mathrm{c}}$ and $\mathrm{H}_{\mathrm{b}}$ should be confirmed. As a result, the absolute configuration of the compound 5 should be determined as (1R,2S,3R)-3-((S)-1-hydroxy-1-phenylethyl)cyclohexane-1,2-diol.
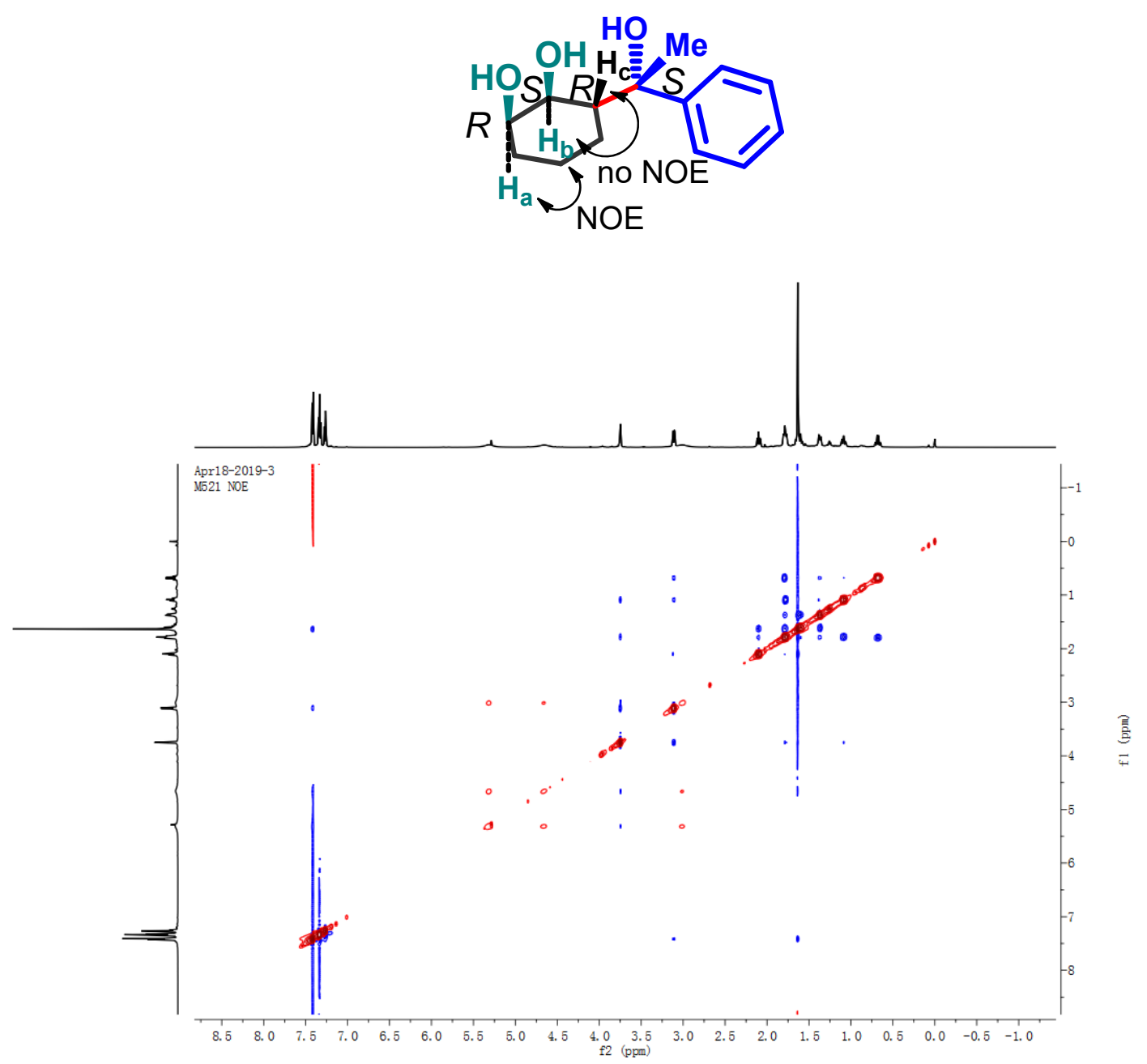
3. Jacques, R.; Pullin, R. D.C.; Fletcher, S. P. Nat. Commun. 2019, doi.org/10.1038/s41467-018-07871-x. 


\section{Analytical data of new compounds}

\section{(2S,3R)-3-methyl-2,4-diphenylpent-4-en-2-ol}<smiles>C=C(c1ccccc1)C(C)C(C)(O)c1ccccc1</smiles>

$3 a$

Prepared according to general procedure. In a nitrogen-filled glovebox, a flame-dried screw-cap reaction tube equipped with a Teflon-coated magnetic stir bar were charged with $\mathrm{Cu}(\mathrm{OAc})_{2}(1.8 \mathrm{mg}, 10.0 \mu \mathrm{mol}),(R, R)-\mathrm{Ph}-\mathrm{BPE}(6.1$ $\mathrm{mg}, 12.0 \mu \mathrm{mol})$, and anhydrous THF (1 mL), dimethoxymethylsilane (DMMS, 3 equiv) were subsequently added. The resulting mixture was stirred for 2 min and then buta-1,3-dien2-ylbenzene (52.0 mg, $0.4 \mathrm{mmol})$ and acetophenone $(24.0 \mathrm{mg}, 0.2 \mathrm{mmol})$ were added, respectively. The vial was removed from the glove box, and the mixture was stirred at $20{ }^{\circ} \mathrm{C}$ for $18 \mathrm{~h}$. After $18 \mathrm{~h}$ the cap was removed. The solution was quenched by adding saturated ammonium fluoride in methanol using a $5 \mathrm{~mL}$ syringe $(3 \mathrm{~mL})$ and stirred for $30 \mathrm{~min}$. After removal of solvent under reduced pressure with aid of a rotary evaporator, the diastereomeric ratio (dr) was assessed by ${ }^{1} \mathrm{H} \mathrm{NMR}$ in $\mathrm{CDCl}_{3}$, The resulting crude product was purified by flash column chromatography on silica gel (diethyl ether /petroleum ether, 1:10) to obtain product in 90\% (45.4 mg) yield as a pale yellow oil. ${ }^{\mathbf{1}} \mathbf{H} \mathbf{~ N M R}\left(600 \mathrm{MHz}, \mathrm{CDCl}_{3}\right) 7.44$ $-7.42(\mathrm{~m}, 4 \mathrm{H}), 7.36-7.27(\mathrm{~m}, 5 \mathrm{H}), 7.21(\mathrm{t}, J=7.2 \mathrm{~Hz}, 1 \mathrm{H}), 5.46(\mathrm{~s}, 1 \mathrm{H}), 5.28(\mathrm{~s}, 1 \mathrm{H}), 3.27$ (q, $J=7.2$ $\mathrm{Hz}, 1 \mathrm{H}), 2.00(\mathrm{~s}, 1 \mathrm{H}), 1.32(\mathrm{~s}, 3 \mathrm{H}), 1.01(\mathrm{~d}, J=7.2 \mathrm{~Hz}, 3 \mathrm{H}) .{ }^{13} \mathbf{C} \mathbf{N M R}\left(150 \mathrm{MHz}, \mathrm{CDCl}_{3}\right) \delta 152.0$, 147.1, 144.8, 128.5, 127.9, 127.3, 126.4, 126.3, 124.9, 115.4, 76.1, 48.2, 30.8, 16.4. HRMS (ESI-TOF) (m/z): Calcd for: $\mathrm{C}_{18} \mathrm{H}_{20} \mathrm{ONa}$ : 275.1407; found: 275.1408 . Specific rotation $[\alpha]^{13}=-36.8,(\mathrm{c}=1.00$, $\mathrm{CHCl}_{3}$ ). HPLC analysis (IA, Hexane/IPA = 98/2, $0.5 \mathrm{~mL} / \mathrm{min}, 250 \mathrm{~nm}$ ) indicated 93\% ee: $\mathrm{tR}$ (maior) $=$ $16.13 \mathrm{~min}, \mathrm{tR}($ minor $)=18.78 \mathrm{~min}$.
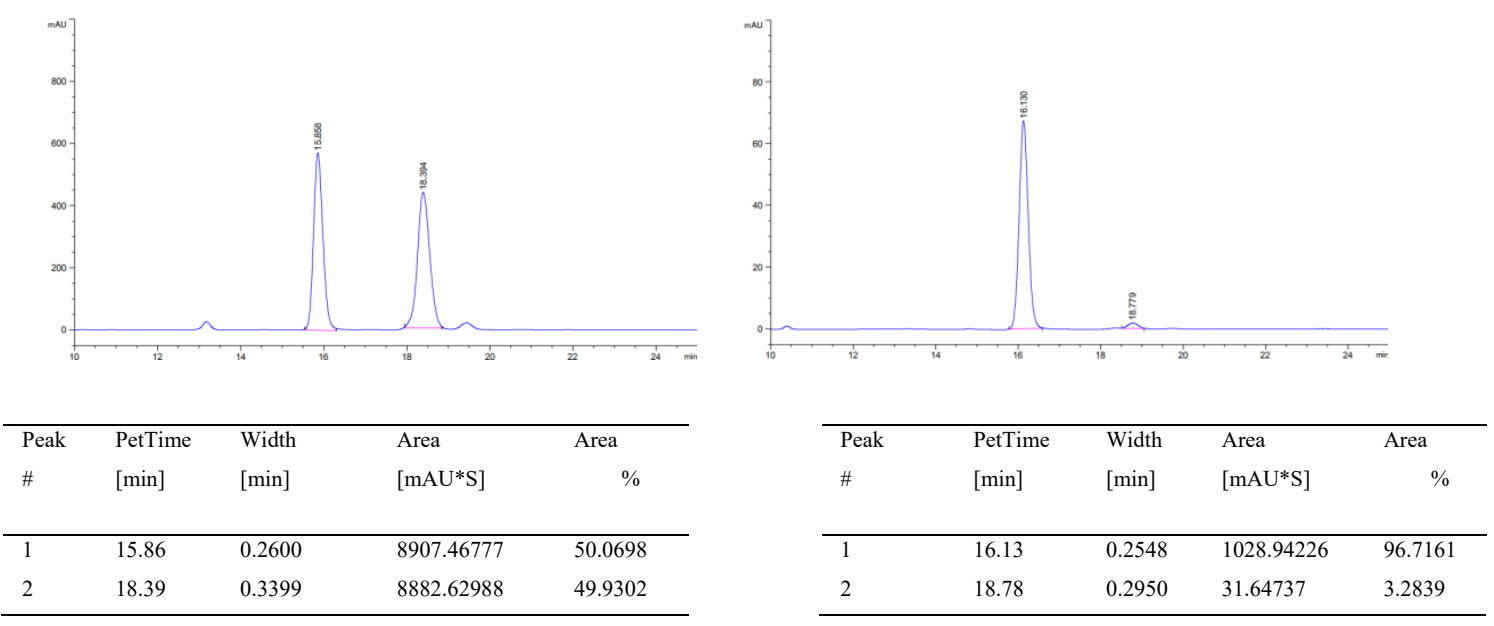

(2S,3R)-2-(4-methoxyphenyl)-3-methyl-4-phenylpent-4-en-2-ol 


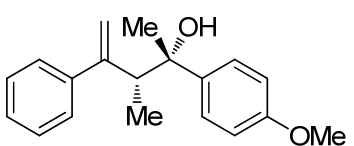

3b

Prepared according to general procedure. In a nitrogen-filled glovebox, a flame-dried screw-cap reaction tube equipped with a Teflon-coated magnetic stir bar were charged with $\mathrm{Cu}(\mathrm{OAc})_{2}(1.8 \mathrm{mg}, 10.0 \mu \mathrm{mol}),(R, R)-$ Ph-BPE (6.1 mg, $12.0 \mu \mathrm{mol})$, and anhydrous THF $(1 \mathrm{~mL})$, dimethoxymethylsilane (DMMS, 3 equiv) were subsequently added. The resulting mixture was stirred for $2 \mathrm{~min}$ and then buta-1,3-dien-2-ylbenzene (52.0 $\mathrm{mg}, 0.4 \mathrm{mmol})$ and 1(4-methoxyphenyl) ethanone ( $30.0 \mathrm{mg}, 0.2 \mathrm{mmol}$ ) were added, respectively. The vial was removed from the glove box, and the mixture was stirred at $20{ }^{\circ} \mathrm{C}$ for $18 \mathrm{~h}$. After $18 \mathrm{~h}$ the cap was removed. The solution was quenched by adding saturated ammonium fluoride in methanol using a $5 \mathrm{~mL}$ syringe $(3 \mathrm{~mL})$ and stirred for $30 \mathrm{~min}$. After removal of solvent under reduced pressure with aid of a rotary evaporator, the diastereomeric ratio (dr) was assessed by ${ }^{1} \mathrm{H} N M R$ in $\mathrm{CDCl}_{3}$, The resulting crude product was purified by flash column chromatography on silica gel (diethyl ether /petroleum ether, 1:10) to obtain product in 94\% (53.0 mg) yield as a colorless oil. ${ }^{1} \mathbf{H}$ NMR $\left(600 \mathrm{MHz}, \mathrm{CDCl}_{3}\right) \delta 7.41-7.40(\mathrm{~m}, 2 \mathrm{H}), 7.35-7.32$ (m, 4H), $7.26(\mathrm{t}, J=7.2 \mathrm{~Hz}, 1 \mathrm{H}), 6.84(\mathrm{~d}, J=9.0 \mathrm{~Hz}, 2 \mathrm{H}), 5.43(\mathrm{~s}, 1 \mathrm{H}), 5.25(\mathrm{~s}, 1 \mathrm{H}), 3.79(\mathrm{~s}, 3 \mathrm{H}), 3.22$ (q, $J=7.2 \mathrm{~Hz}, 1 \mathrm{H}), 1.98$ (s, 1H), 1.31 (s, $3 \mathrm{H}), 1.02$ (d, $J=7.2 \mathrm{~Hz}, 3 \mathrm{H}) .{ }^{13} \mathbf{C}$ NMR $\left(150 \mathrm{MHz}, \mathrm{CDCl}_{3}\right) \delta$ 158.0, 152.0, 144.9, 139.3, 128.4, 127.2, 126.4, 126.0, 115.2, 113.2, 75.9, 55.2, 48.4, 30.6, 16.5. HRMS (ESI-TOF) $(\mathrm{m} / \mathrm{z})$ : Calcd for $\mathrm{C}_{19} \mathrm{H}_{22} \mathrm{O}_{2} \mathrm{Na}\left([\mathrm{M}+\mathrm{Na}]^{+}\right)$: 305.1512 ; found: 305.1515 . Specific rotation $[\alpha]^{15} \mathrm{D}=-29.5,\left(\mathrm{c}=1.00, \mathrm{CHCl}_{3}\right)$. HPLC analysis (IA, Hexane $/ \mathrm{IPA}=98 / 2,0.5 \mathrm{~mL} / \mathrm{min}, 250 \mathrm{~nm}$ ) indicated $93 \%$ ee: $\mathrm{tR}($ major $)=22.56 \mathrm{~min}, \mathrm{tR}($ minor $)=31.10 \mathrm{~min}$.
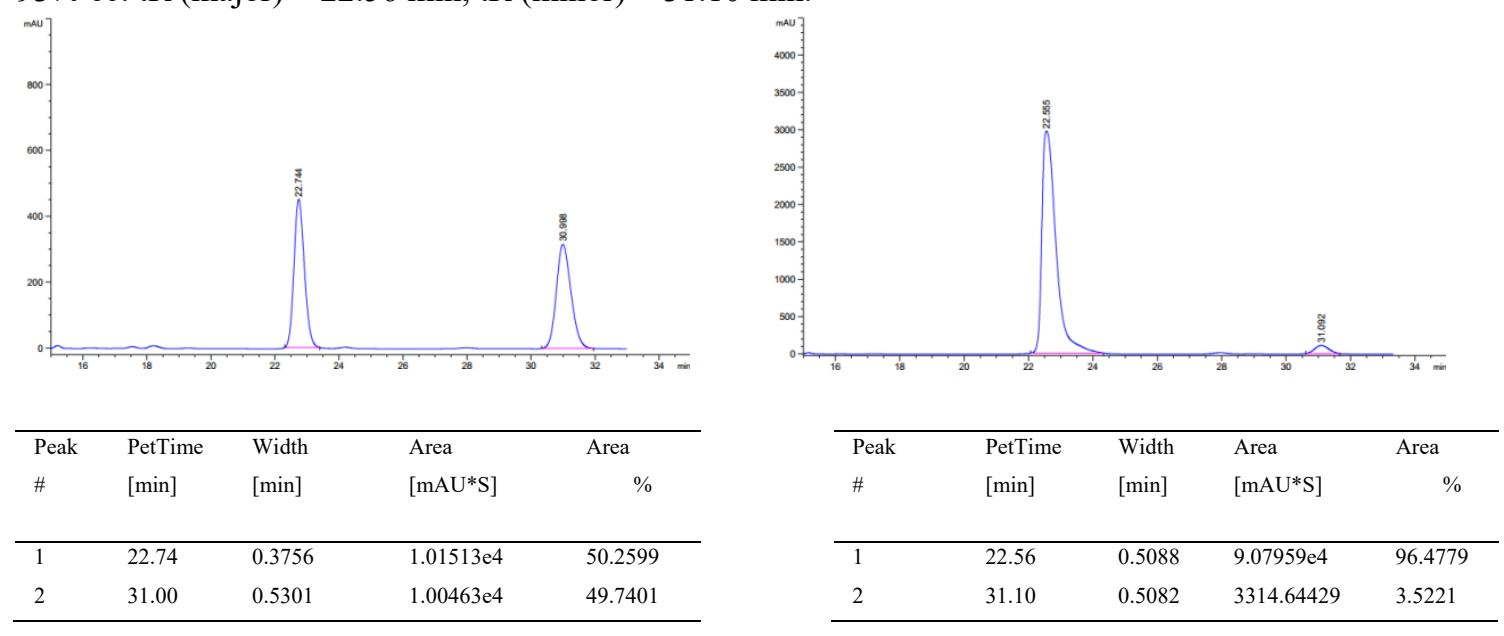

\section{(2S,3R)-2-(3-methoxyphenyl)-3-methyl-4-phenylpent-4-en-2-ol}

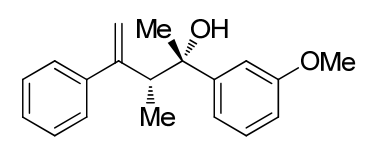

$3 c$

Prepared according to general procedure. In a nitrogen-filled glovebox, a flame-dried screw-cap reaction tube equipped with a Teflon-coated magnetic stir bar were charged with $\mathrm{Cu}(\mathrm{OAc})_{2}(1.8 \mathrm{mg}, 10.0 \mu \mathrm{mol}),(R, R)-$ $\mathrm{Ph}-\mathrm{BPE} \quad(6.1 \mathrm{mg}, \quad 12.0 \mu \mathrm{mol})$, and anhydrous THF $(1 \mathrm{~mL})$, dimethoxymethylsilane (DMMS, 3 equiv) were subsequently added. The resulting mixture was stirred for $2 \mathrm{~min}$ and then buta-1,3-dien-2-ylbenzene (52.0 mg, $0.4 \mathrm{mmol}$ ) and 1-(3-methoxyphenyl) ethanone (30.0 $\mathrm{mg}, 0.2 \mathrm{mmol}$ ) were added, respectively. The vial was removed from the glove box, and the mixture was stirred at $20{ }^{\circ} \mathrm{C}$ for $18 \mathrm{~h}$. After $18 \mathrm{~h}$ the cap was removed. The solution was quenched by adding saturated ammonium fluoride in methanol using a $5 \mathrm{~mL}$ syringe $(3 \mathrm{~mL})$ and stirred for $30 \mathrm{~min}$. After 
removal of solvent under reduced pressure with aid of a rotary evaporator, the diastereomeric ratio (dr) was assessed by ${ }^{1} \mathrm{H}$ NMR in $\mathrm{CDCl}_{3}$, The resulting crude product was purified by flash column chromatography on silica gel (diethyl ether /petroleum ether, 1:10) to obtain product in $86 \%(48.5 \mathrm{mg})$ yield as a colorless oil. ${ }^{1} \mathbf{H}$ NMR $\left(600 \mathrm{MHz}, \mathrm{CDCl}_{3}\right) \delta 7.41(\mathrm{~d}, J=7.8 \mathrm{~Hz}, 2 \mathrm{H}), 7.34(\mathrm{t}, J=7.8 \mathrm{~Hz}, 2 \mathrm{H})$, $7.28(\mathrm{~d}, J=7.2 \mathrm{~Hz}, 1 \mathrm{H}), 7.23$ (t, $J=7.8 \mathrm{~Hz}, 1 \mathrm{H}), 7.04(\mathrm{~d}, J=2.4 \mathrm{~Hz}, 1 \mathrm{H}), 6.99$ (d, $J=7.8 \mathrm{~Hz}, 1 \mathrm{H}), 6.75$ (dd, $J=8.1,2.4 \mathrm{~Hz}, 1 \mathrm{H}), 5.45(\mathrm{~s}, 1 \mathrm{H}), 5.28(\mathrm{~s}, 1 \mathrm{H}), 3.80(\mathrm{~s}, 3 \mathrm{H}), 3.25(\mathrm{q}, J=7.2 \mathrm{~Hz}, 1 \mathrm{H}), 2.01(\mathrm{~s}, 1 \mathrm{H})$, $1.30(\mathrm{~s}, 3 \mathrm{H}), 1.02(\mathrm{~d}, J=7.2 \mathrm{~Hz}, 3 \mathrm{H}) .{ }^{13} \mathbf{C}$ NMR $\left(150 \mathrm{MHz}, \mathrm{CDCl}_{3}\right) \delta 159.4,151.9,149.0,144.79,128.90$, 128.4, 127.2, 126.4, 117.3, 115.4,111.22, 111.23, 76.1, 55.2, 48.1, 30.7, 16.4. HRMS (ESI-TOF) (m/z): Calcd for $\mathrm{C}_{19} \mathrm{H}_{22} \mathrm{O}_{2} \mathrm{Na}\left([\mathrm{M}+\mathrm{Na}]^{+}\right)$: 305.1512 ; found: 305.1522 . Specific rotation $[\alpha]^{13} \mathrm{D}=-26.3$, $(\mathrm{c}$ $=1.00, \mathrm{CHCl}_{3}$ ). HPLC analysis (IA, Hexane/IPA $=98 / 2,0.5 \mathrm{~mL} / \mathrm{min}, 250 \mathrm{~nm}$ ) indicated $94 \%$ ee: $\mathrm{tR}$ $($ major $)=24.02 \mathrm{~min}, \mathrm{tR}($ minor $)=33.11 \mathrm{~min}$.
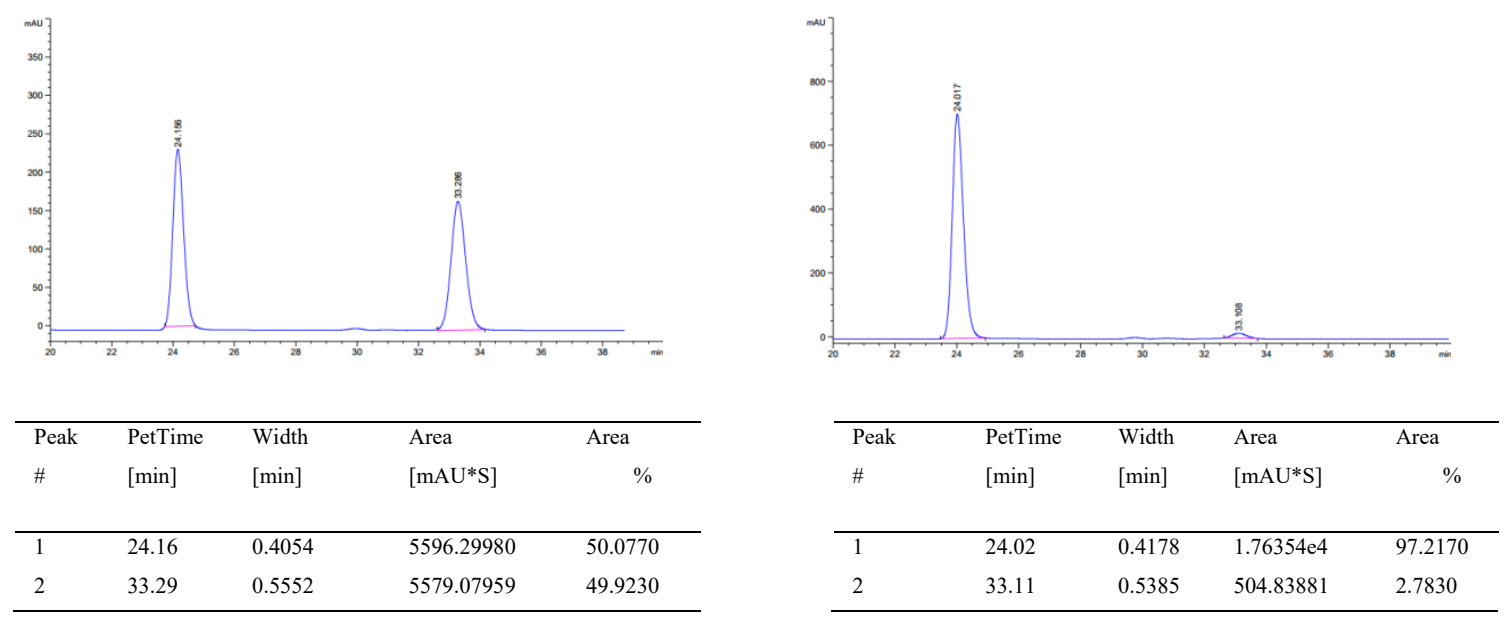

\section{(2S,3R)-2-(2-methoxyphenyl)-3-methyl-4-phenylpent-4-en-2-ol}

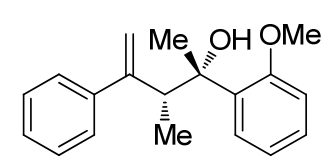

3d

Prepared according to general procedure. In a nitrogen-filled glovebox, a flamedried screw-cap reaction tube equipped with a Teflon-coated magnetic stir bar were charged with $\mathrm{Cu}(\mathrm{OAc})_{2}(1.8 \mathrm{mg}, 10.0 \mu \mathrm{mol}),(R, R)-\mathrm{Ph}-\mathrm{BPE}(6.1 \mathrm{mg}, 12.0$ $\mu \mathrm{mol})$, and anhydrous THF ( $1 \mathrm{~mL}$ ), dimethoxymethylsilane (DMMS, 3 equiv) were subsequently added. The resulting mixture was stirred for $2 \mathrm{~min}$ and then

buta-1,3-dien-2-ylbenzene ( $52.0 \mathrm{mg}, 0.4 \mathrm{mmol})$ and 1-(2-methoxyphenyl) ethanone ( $30.0 \mathrm{mg}, 0.2 \mathrm{mmol})$ were added, respectively. The vial was removed from the glove box, and the mixture was stirred at $20{ }^{\circ} \mathrm{C}$ for $18 \mathrm{~h}$. After $18 \mathrm{~h}$ the cap was removed. The solution was quenched by adding saturated ammonium fluoride in methanol using a $5 \mathrm{~mL}$ syringe $(3 \mathrm{~mL})$ and stirred for $30 \mathrm{~min}$. After removal of solvent under reduced pressure with aid of a rotary evaporator, the diastereomeric ratio (dr) was assessed by ${ }^{1} \mathrm{H}$ NMR in $\mathrm{CDCl}_{3}$, The resulting crude product was purified by flash column chromatography on silica gel (diethyl ether /petroleum ether, 1:10) to obtain product in $93 \%(52.5 \mathrm{mg})$ yield as a white solid. m.p. $59-60{ }^{\circ} \mathrm{C}$. ${ }^{1} \mathbf{H}$ NMR $\left(600 \mathrm{MHz}, \mathrm{CDCl}_{3}\right) \delta 7.19(\mathrm{~d}, J=7.2 \mathrm{~Hz}, 1 \mathrm{H}), 7.05-7.00(\mathrm{~m}, 5 \mathrm{H}), 6.97-6.94(\mathrm{~m}, 1 \mathrm{H}), 6.76$ $(\mathrm{t}, J=7.2 \mathrm{~Hz}, 1 \mathrm{H}), 6.46(\mathrm{~d}, J=8.4 \mathrm{~Hz}, 1 \mathrm{H}), 5.18(\mathrm{~s}, 1 \mathrm{H}), 5.14(\mathrm{~s}, 1 \mathrm{H}), 3.73(\mathrm{q}, J=7.2 \mathrm{~Hz}, 1 \mathrm{H}), 3.60(\mathrm{~s}$, $3 \mathrm{H}), 3.53(\mathrm{~s}, 1 \mathrm{H}), 1.39(\mathrm{~s}, 3 \mathrm{H}), 1.15(\mathrm{~d}, J=7.2 \mathrm{~Hz}, 3 \mathrm{H}) .{ }^{13} \mathbf{C} \mathbf{~ N M R}\left(150 \mathrm{MHz}, \mathrm{CDCl}_{3}\right) \delta 156.3,152.3$, 144.5, 134.2, 127.9, 127.6, 127.3, 126.4, 126.2, 120.5, 114.3, 110.5, 76.7, 54.9, 45.1, 23.8, 16.1. HRMS (ESI-TOF) $(\mathrm{m} / \mathrm{z})$ : Calcd for $\mathrm{C}_{19} \mathrm{H}_{22} \mathrm{O}_{2} \mathrm{Na}\left([\mathrm{M}+\mathrm{Na}]^{+}\right): 305.1512$; found: 305.1511 . Specific rotation $[\alpha]^{13} \mathrm{D}=-36.4,\left(\mathrm{c}=1.00, \mathrm{CHCl}_{3}\right)$. HPLC analysis $(\mathrm{IB}, \mathrm{Hexane} / \mathrm{IPA}=99 / 1,0.5 \mathrm{~mL} / \mathrm{min}, 250 \mathrm{~nm})$ indicated $86 \%$ ee: $\mathrm{tR}($ major $)=26.90 \mathrm{~min}, \mathrm{tR}($ minor $)=19.27 \mathrm{~min}$. 

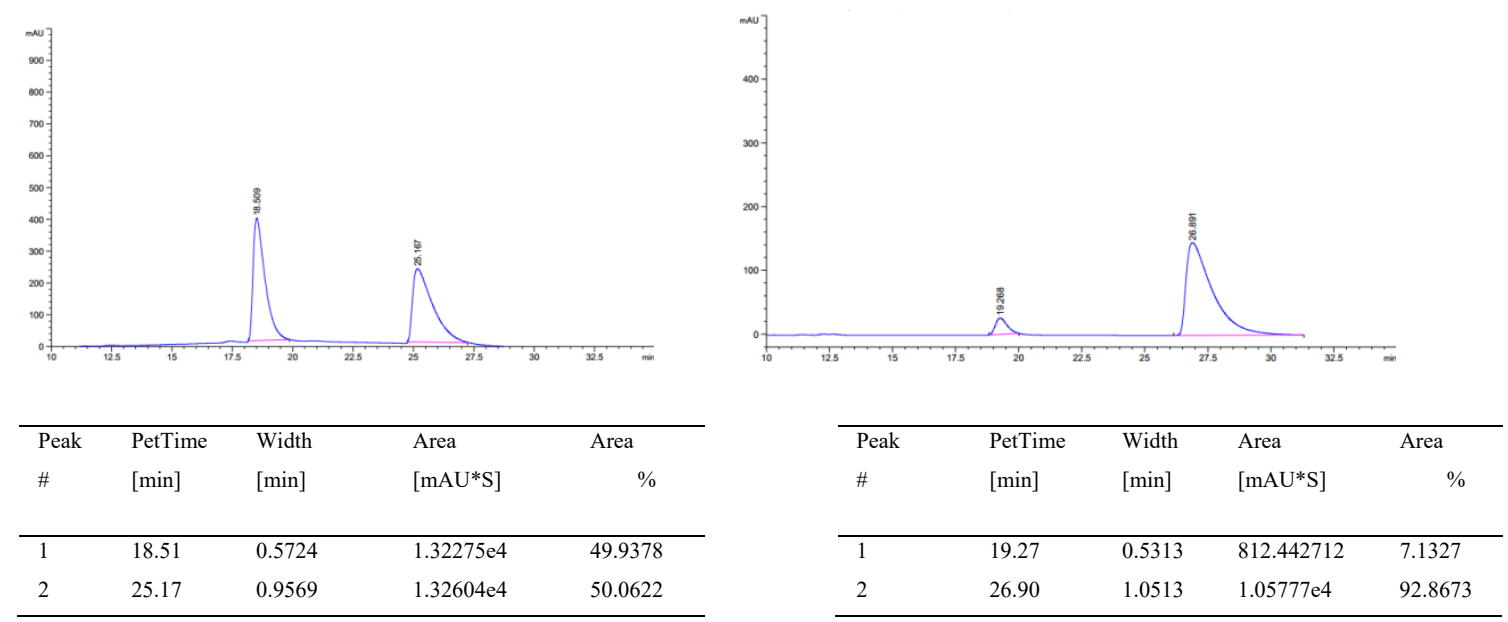

\section{(2S,3R)-2-(benzo[d][1,3]dioxol-5-yl)-3-methyl-4-phenylpent-4-en-2-ol}<smiles>C=C(c1ccccc1)[C@H](C)[C@](C)(O)c1ccc2c(c1)OCO2</smiles>

$3 e$

Prepared according to general procedure. In a nitrogen-filled glovebox, a flame-dried screw-cap reaction tube equipped with a Teflon-coated magnetic stir bar were charged with $\mathrm{Cu}(\mathrm{OAc})_{2}(1.8 \mathrm{mg}, 10.0 \mu \mathrm{mol}),(R, R)-$ Ph-BPE (6.1 mg, $12.0 \mu \mathrm{mol})$, and anhydrous THF (1 mL), dimethoxymethylsilane (DMMS, 3 equiv) were subsequently added. The resulting mixture was stirred for $2 \mathrm{~min}$ and then buta-1,3-dien-2-ylbenzene $(52.0 \mathrm{mg}, 0.4 \mathrm{mmol})$ and 1(benzo[d][1,3]dioxol-5-yl) ethanone $(32.8 \mathrm{mg}, 0.2 \mathrm{mmol})$ were added, respectively. The vial was removed from the glove box, and the mixture was stirred at $20{ }^{\circ} \mathrm{C}$ for $18 \mathrm{~h}$. After $18 \mathrm{~h}$ the cap was removed. The solution was quenched by adding saturated ammonium fluoride in methanol using a $5 \mathrm{~mL}$ syringe $(3 \mathrm{~mL})$ and stirred for $30 \mathrm{~min}$. After removal of solvent under reduced pressure with aid of a rotary evaporator, the diastereomeric ratio (dr) was assessed by ${ }^{1} \mathrm{H} \mathrm{NMR}$ in $\mathrm{CDCl}_{3}$, The resulting crude product was purified by flash column chromatography on silica gel (diethyl ether /petroleum ether, 1:10) to obtain product in $93 \%(55.1 \mathrm{mg})$ yield as a colorless oil. ${ }^{1} \mathbf{H} \mathbf{~ N M R}\left(600 \mathrm{MHz}, \mathrm{CDCl}_{3}\right) \delta 7.39(\mathrm{~d}, J=$ $7.8 \mathrm{~Hz}, 2 \mathrm{H}), 7.33(\mathrm{t}, J=7.8 \mathrm{~Hz}, 2 \mathrm{H}), 7.27-7.25(\mathrm{~m}, 1 \mathrm{H}), 6.94(\mathrm{~s}, 1 \mathrm{H}), 6.89-6.88(\mathrm{~m}, 1 \mathrm{H}), 6.74(\mathrm{~d}, J=$ $7.8 \mathrm{~Hz}, 1 \mathrm{H}), 5.92(\mathrm{~s}, 2 \mathrm{H}), 5.43$ (s, 1H), $5.26(\mathrm{~s}, 1 \mathrm{H}), 3.20$ (q, $J=7.2 \mathrm{~Hz}, 1 \mathrm{H}), 1.98(\mathrm{~s}, 1 \mathrm{H}), 1.28(\mathrm{~s}, 3 \mathrm{H})$, $1.04(\mathrm{~d}, J=7.2 \mathrm{~Hz}, 3 \mathrm{H}) .{ }^{13} \mathbf{C}$ NMR $\left(150 \mathrm{MHz}, \mathrm{CDCl}_{3}\right) \delta 152.0,147.3,145.8,144.8,141.4,128.4,127.2$, 126.3, 118.0, 115.3, 107.6, 106.1, 100.8, 76.0, 48.4, 30.6, 16.4. HRMS (ESI-TOF) (m/z): Calcd for $\mathrm{C}_{19} \mathrm{H}_{22} \mathrm{O}_{3} \mathrm{Na}\left([\mathrm{M}+\mathrm{Na}]^{+}\right): 321.1461$; found: 321.1454 . Specific rotation $[\alpha]^{13} \mathrm{D}=-29.4,\left(\mathrm{c}=1.00, \mathrm{CHCl}_{3}\right)$. HPLC analysis (IA, Hexane/IPA $=98 / 2,0.5 \mathrm{~mL} / \mathrm{min}, 250 \mathrm{~nm}$ ) indicated $93 \%$ ee: $\mathrm{tR}$ (major) $=31.71$ $\min , \mathrm{tR}(\operatorname{minor})=44.12 \mathrm{~min}$.
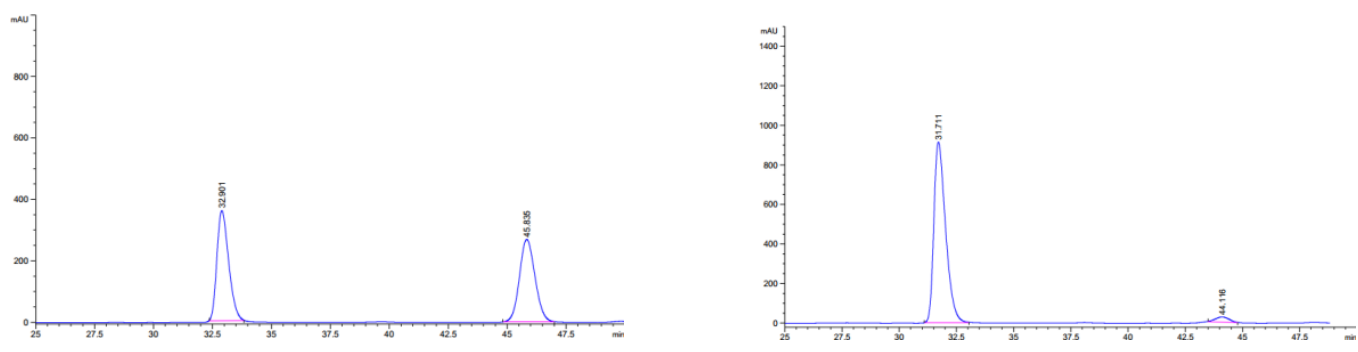


\begin{tabular}{lllll}
\hline $\begin{array}{l}\text { Peak } \\
\#\end{array}$ & $\begin{array}{l}\text { PetTime } \\
{[\mathrm{min}]}\end{array}$ & $\begin{array}{c}\text { Width } \\
{[\mathrm{min}]}\end{array}$ & $\begin{array}{l}\text { Area } \\
{[\mathrm{mAU} * \mathrm{~S}]}\end{array}$ & $\begin{array}{c}\text { Area } \\
\%\end{array}$ \\
\hline 1 & 32.90 & 0.5776 & $1.24081 \mathrm{e} 4$ & 50.2310 \\
2 & 45.84 & 0.7632 & $1.22939 \mathrm{e} 4$ & 49.7690 \\
\hline
\end{tabular}

\begin{tabular}{lllll}
\hline $\begin{array}{l}\text { Peak } \\
\#\end{array}$ & $\begin{array}{l}\text { PetTime } \\
{[\mathrm{min}]}\end{array}$ & $\begin{array}{l}\text { Width } \\
{[\mathrm{min}]}\end{array}$ & $\begin{array}{l}\text { Area } \\
{[\mathrm{mAU} * \mathrm{~S}]}\end{array}$ & $\begin{array}{c}\text { Area } \\
\%\end{array}$ \\
\hline 1 & 31.71 & 0.5936 & $3.25289 \mathrm{e} 4$ & 96.6937 \\
2 & 44.12 & 0.6985 & 1112.28198 & 3.3063 \\
\hline
\end{tabular}

\section{4-((2S,3R)-2-hydroxy-3-methyl-4-phenylpent-4-en-2-yl)-2-methylphenol}<smiles>[Y19]C(O)(C(=C)c1ccccc1)c1ccc(O)c(C(C)(O)O)c1</smiles>
resulting mixture was stirred for $2 \mathrm{~min}$ and then buta-1,3-dien-2-ylbenzene $(52.0 \mathrm{mg}, 0.4 \mathrm{mmol})$ and 1(4-hydroxy-3-methylphenyl) ethanone $(30.0 \mathrm{mg}, 0.2 \mathrm{mmol})$ were added, respectively. The vial was removed from the glove box, and the mixture was stirred at $20{ }^{\circ} \mathrm{C}$ for $18 \mathrm{~h}$. After $18 \mathrm{~h}$ the cap was removed. The solution was quenched by adding saturated ammonium fluoride in methanol using a $5 \mathrm{~mL}$ syringe ( $3 \mathrm{~mL}$ ) and stirred for $30 \mathrm{~min}$. After removal of solvent under reduced pressure with aid of a rotary evaporator, the diastereomeric ratio (dr) was assessed by ${ }^{1} \mathrm{H}$ NMR in $\mathrm{CDCl}_{3}$, The resulting crude product was purified by flash column chromatography on silica gel (diethyl ether /petroleum ether, 1:10) to obtain product in $94 \%(53.0 \mathrm{mg})$ yield as a colorless oil. ${ }^{1} \mathbf{H} \mathbf{~ N M R}\left(600 \mathrm{MHz}, \mathrm{CDCl}_{3}\right) \delta 9.34(\mathrm{~s}, 1 \mathrm{H})$, $7.39-7.38(\mathrm{~m}, 2 \mathrm{H}), 7.34(\mathrm{t}, J=7.6 \mathrm{~Hz}, 2 \mathrm{H}), 7.28(\mathrm{t}, J=7.2 \mathrm{~Hz}, 1 \mathrm{H}), 6.88(\mathrm{dd}, J=8.2,1.7 \mathrm{~Hz}, 1 \mathrm{H})$, 6.71- $6.67(\mathrm{~m}, 2 \mathrm{H}), 5.49(\mathrm{~s}, 1 \mathrm{H}), 5.30(\mathrm{~s}, 1 \mathrm{H}), 3.35(\mathrm{q}, J=7.2 \mathrm{~Hz}, 1 \mathrm{H}), 2.73(\mathrm{~s}, 1 \mathrm{H}), 2.23(\mathrm{~s}, 3 \mathrm{H}), 1.37$ $(\mathrm{s}, 3 \mathrm{H}), 1.23(\mathrm{~d}, J=7.2 \mathrm{~Hz}, 3 \mathrm{H}) .{ }^{13} \mathbf{C}$ NMR $\left(150 \mathrm{MHz}, \mathrm{CDCl}_{3}\right) \delta 153.8,151.6,144.3,129.1,128.5,128.2$, 127.8, 127.5 126.8, 126.3 117.4, 115.7, 81.1, 48.5, 30.4, 20.7, 16.0. HRMS (ESI-TOF) (m/z): Calcd for $\mathrm{C}_{19} \mathrm{H}_{22} \mathrm{O}_{2} \mathrm{Na}\left([\mathrm{M}+\mathrm{Na}]^{+}\right): 305.1512$; found: 305.1505 . Specific rotation $[\alpha]^{13} \mathrm{D}=-32.5,\left(\mathrm{c}=1.00, \mathrm{CHCl}_{3}\right)$. HPLC analysis (IA, Hexane $/ \mathrm{IPA}=98 / 2,0.5 \mathrm{~mL} / \mathrm{min}, 250 \mathrm{~nm}$ ) indicated $93 \%$ ee: $\mathrm{tR}$ (major) $=33.87$ $\min , \mathrm{tR}($ minor $)=38.61 \mathrm{~min}$.
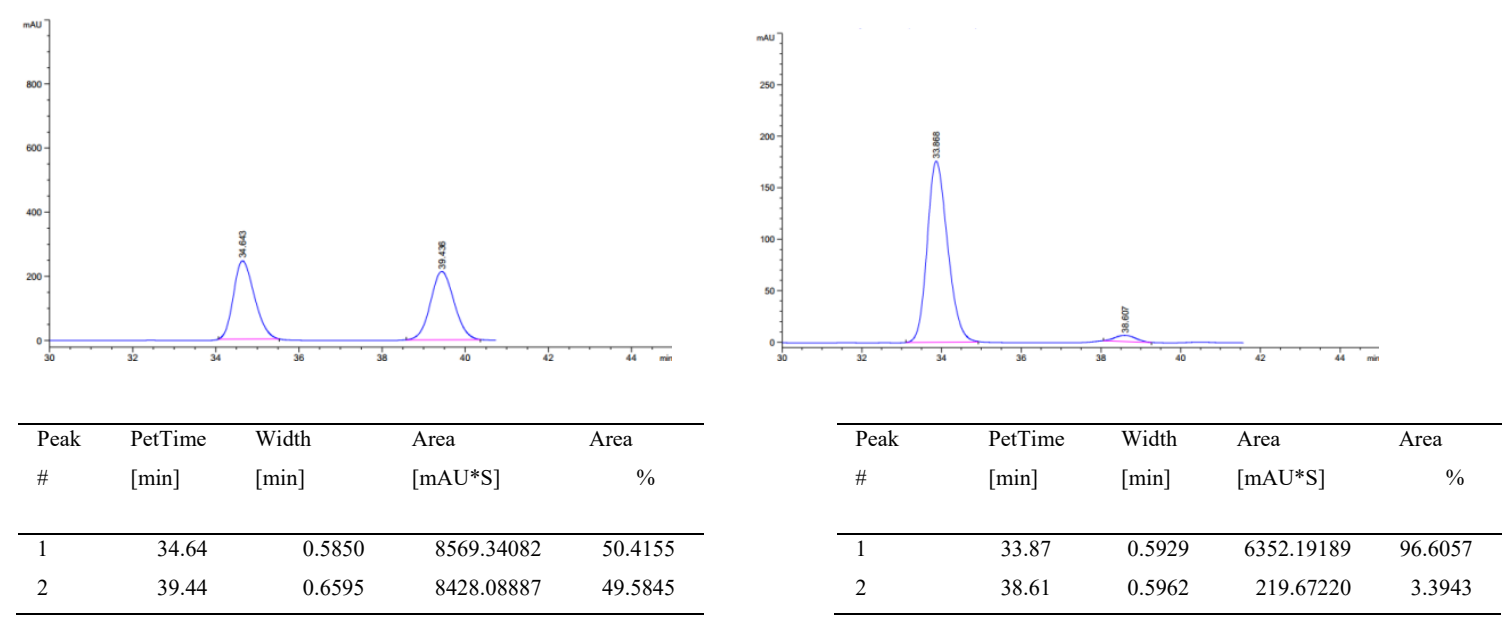

(2S,3R)-3-methyl-4-phenyl-2-(p-tolyl)pent-4-en-2-ol 


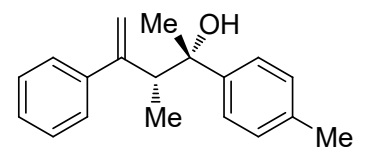

$3 g$

Prepared according to general procedure. In a nitrogen-filled glovebox, a flame-dried screw-cap reaction tube equipped with a Teflon-coated magnetic stir bar were charged with $\mathrm{Cu}(\mathrm{OAc})_{2}(1.8 \mathrm{mg}, 10.0 \mu \mathrm{mol}),(R, R)$ Ph-BPE (6.1 mg, $12.0 \mu \mathrm{mol})$, and anhydrous THF (1 $\mathrm{mL})$, dimethoxymethylsilane (DMMS, 3 equiv) were subsequently added. The resulting mixture was stirred for $2 \mathrm{~min}$ and then buta-1,3-dien-2-ylbenzene $(52.0 \mathrm{mg}, 0.4 \mathrm{mmol})$ and 1( $p$-tolyl) ethanone ( $26.8 \mathrm{mg}, 0.2 \mathrm{mmol})$ were added, respectively. The vial was removed from the glove box, and the mixture was stirred at $20{ }^{\circ} \mathrm{C}$ for $18 \mathrm{~h}$. After $18 \mathrm{~h}$ the cap was removed. The solution was quenched by adding saturated ammonium fluoride in methanol using a $5 \mathrm{~mL}$ syringe $(3 \mathrm{~mL})$ and stirred for $30 \mathrm{~min}$. After removal of solvent under reduced pressure with aid of a rotary evaporator, the diastereomeric ratio (dr) was assessed by ${ }^{1} \mathrm{H} N M R$ in $\mathrm{CDCl}_{3}$, The resulting crude product was purified by flash column chromatography on silica gel (diethyl ether /petroleum ether, 1:10) to obtain product in 91\% (48.5 mg) yield as a colorless oil. ${ }^{1} \mathbf{H}$ NMR $\left(600 \mathrm{MHz}, \mathrm{CDCl}_{3}\right) \delta 7.43-7.42(\mathrm{~m}, 2 \mathrm{H}), 7.36-7.31$ (m, 4H), $7.29-7.26(\mathrm{~m}, 1 \mathrm{H}), 7.12$ (d, $J=8.4 \mathrm{~Hz}, 2 \mathrm{H}), 5.44$ (s, 1H), 5.27 (s, 1H), 3.24 (q, J = 7.2 Hz, 1H), 2.33 (s, 3H), 1.97 (s, 1H), 1.30 (s, 3H), 1.01 (d, $J=7.2 \mathrm{~Hz}, 3 \mathrm{H}) .{ }^{13} \mathbf{C} \mathbf{~ N M R}\left(\mathbf{1 5 0} \mathbf{~ M H z}, \mathbf{C D C l}_{3}\right) \delta$ 152.1, 144.9, 144.2, 135.8, 128.6, 128.4, 127.2, 126.4, 124.8, 115.3, 76.1, 48.2, 30.9, 20.93, 16.5. HRMS (ESI-TOF) $(\mathrm{m} / \mathrm{z})$ : Calcd for $\mathrm{C}_{19} \mathrm{H}_{22} \mathrm{ONa}\left([\mathrm{M}+\mathrm{Na}]^{+}\right)$: 289.1563; found: 289.1562 . Specific rotation $[\alpha]^{14} \mathrm{D}=-27.5,\left(\mathrm{c}=1.00, \mathrm{CHCl}_{3}\right)$. HPLC analysis (IA, Hexane $\left./ \mathrm{IPA}=98 / 2,0.5 \mathrm{~mL} / \mathrm{min}, 250 \mathrm{~nm}\right)$ indicated $90 \%$ ee: $\mathrm{tR}($ major $)=12.30 \mathrm{~min}, \mathrm{tR}($ minor $)=15.18 \mathrm{~min}$.

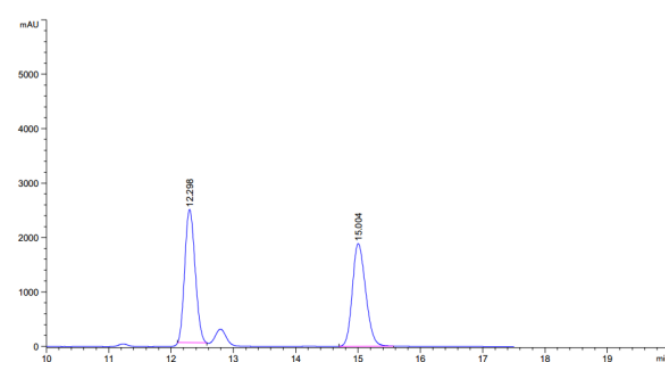

\begin{tabular}{llllc}
\hline $\begin{array}{l}\text { Peak } \\
\#\end{array}$ & $\begin{array}{l}\text { PetTime } \\
{[\mathrm{min}]}\end{array}$ & $\begin{array}{c}\text { Width } \\
{[\mathrm{min}]}\end{array}$ & $\begin{array}{l}\text { Area } \\
{[\mathrm{mAU} * \mathrm{~S}]}\end{array}$ & $\begin{array}{c}\text { Area } \\
\%\end{array}$ \\
\hline 1 & 12.30 & 0.1928 & $2.82891 \mathrm{e} 4$ & 50.2839 \\
2 & 15.04 & 0.2469 & $2.79697 \mathrm{e} 4$ & 49.7161 \\
\hline
\end{tabular}

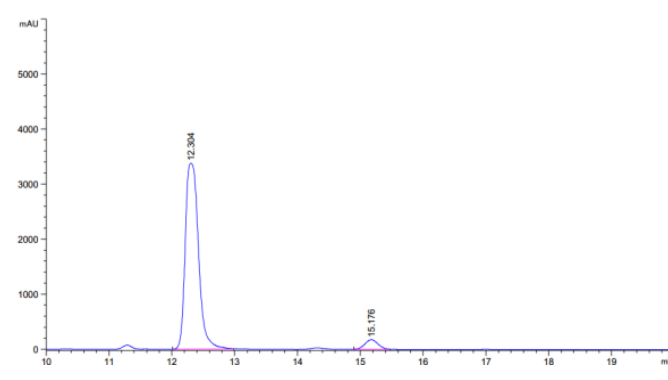

\begin{tabular}{lllll}
\hline $\begin{array}{l}\text { Peak } \\
\#\end{array}$ & $\begin{array}{l}\text { PetTime } \\
{[\mathrm{min}]}\end{array}$ & $\begin{array}{l}\text { Width } \\
{[\mathrm{min}]}\end{array}$ & $\begin{array}{l}\text { Area } \\
{\left[\mathrm{mAU}^{*} \mathrm{~S}\right]}\end{array}$ & $\begin{array}{c}\text { Area } \\
\%\end{array}$ \\
\hline 1 & 12.30 & 0.2432 & $4.93018 \mathrm{e} 4$ & 95.1388 \\
2 & 15.18 & 0.2402 & 2519.11719 & 4.8612 \\
\hline
\end{tabular}

(2S,3R)-3-methyl-4-phenyl-2-(o-tolyl)pent-4-en-2-ol<smiles>C=C(c1ccccc1)[C@H](C)[C@](C)(O)c1ccccc1C</smiles>

$3 \mathrm{~h}$

Prepared according to general procedure. In a nitrogen-filled glovebox, a flamedried screw-cap reaction tube equipped with a Teflon-coated magnetic stir bar were charged with $\mathrm{Cu}(\mathrm{OAc})_{2}(1.8 \mathrm{mg}, 10.0 \mu \mathrm{mol}),(R, R)-\mathrm{Ph}-\mathrm{BPE}(6.1 \mathrm{mg}, 12.0$ $\mu \mathrm{mol})$, and anhydrous THF ( $1 \mathrm{~mL}$ ), dimethoxymethylsilane (DMMS, 3 equiv) were subsequently added. The resulting mixture was stirred for $2 \mathrm{~min}$ and then buta-1,3-dien-2-ylbenzene (52.0 mg, $0.4 \mathrm{mmol})$ and 1-(o-tolyl) ethanone $(26.8 \mathrm{mg}, 0.2 \mathrm{mmol})$ were added, respectively. The vial was removed from the glove box, and the mixture was stirred at $20{ }^{\circ} \mathrm{C}$ for $18 \mathrm{~h}$. After $18 \mathrm{~h}$ the cap was 
removed. The solution was quenched by adding saturated ammonium fluoride in methanol using a $5 \mathrm{~mL}$ syringe $(3 \mathrm{~mL})$ and stirred for $30 \mathrm{~min}$. After removal of solvent under reduced pressure with aid of a rotary evaporator, the diastereomeric ratio (dr) was assessed by ${ }^{1} \mathrm{H} \mathrm{NMR}$ in $\mathrm{CDCl}_{3}$, The resulting crude product was purified by flash column chromatography on silica gel (diethyl ether /petroleum ether, 1:10) to obtain product in $94 \%(50.1 \mathrm{mg})$ yield as a colorless oil. ${ }^{1} \mathbf{H}$ NMR $\left(600 \mathrm{MHz}, \mathrm{CDCl}_{3}\right) \delta 7.59(\mathrm{~d}, J=$ $7.8 \mathrm{~Hz}, 1 \mathrm{H}), 7.36(\mathrm{~d}, J=7.8 \mathrm{~Hz}, 2 \mathrm{H}), 7.31(\mathrm{t}, J=7.2 \mathrm{~Hz}, 2 \mathrm{H}), 7.26-7.23(\mathrm{~m}, 1 \mathrm{H}), 7.14(\mathrm{t}, J=7.2 \mathrm{~Hz}$, 1H), 7.08 (t, $J=7.2 \mathrm{~Hz}, 1 \mathrm{H}), 7.04$ (d, $J=7.2 \mathrm{~Hz}, 1 \mathrm{H}), 5.45(\mathrm{~s}, 1 \mathrm{H}), 5.33$ (s, 1H), 3.62 (q, $J=7.2 \mathrm{~Hz}$, 1H), $2.46(\mathrm{~s}, 3 \mathrm{H}), 2.03(\mathrm{~s}, 1 \mathrm{H}), 1.35(\mathrm{~s}, 3 \mathrm{H}), 1.06(\mathrm{~d}, J=7.2 \mathrm{~Hz}, 3 \mathrm{H}) .{ }^{13} \mathbf{C} \mathbf{N M R}\left(150 \mathrm{MHz}, \mathrm{CDCl}_{3}\right) \delta$ 152.2, 144.8, 144.5, 134.0, 132.3, 128.4, 127.2, 126.6, 126.6, 126.3, 125.6, 115.8, 76.8, 45.3, 29.3, 22.6, 16.3. HRMS (ESI-TOF) $(\mathrm{m} / \mathrm{z})$ : Calcd for $\mathrm{C}_{19} \mathrm{H}_{22} \mathrm{ONa}\left([\mathrm{M}+\mathrm{Na}]^{+}\right): 289.1563$; found: 289.1563. Specific rotation $[\alpha]^{14}{ }_{\mathrm{D}}=-29.4,\left(\mathrm{c}=1.00, \mathrm{CHCl}_{3}\right)$. HPLC analysis (IA, Hexane/IPA = 98/2, $\left.0.5 \mathrm{~mL} / \mathrm{min}, 250 \mathrm{~nm}\right)$ indicated 94\% ee: $\mathrm{tR}$ (major) $=12.67 \mathrm{~min}, \mathrm{tR}($ minor $)=14.65 \mathrm{~min}$.
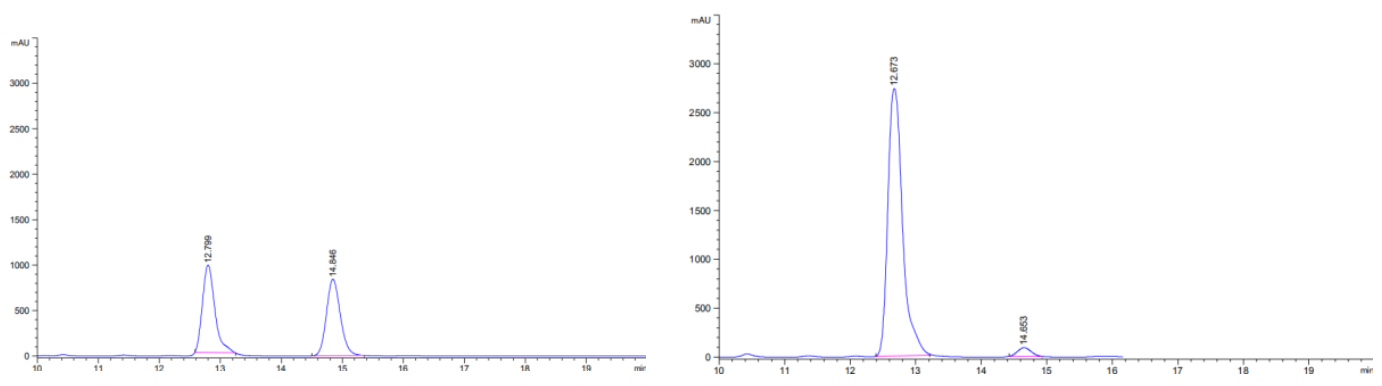

\begin{tabular}{lllll}
\hline $\begin{array}{l}\text { Peak } \\
\#\end{array}$ & $\begin{array}{l}\text { PetTime } \\
{[\mathrm{min}]}\end{array}$ & $\begin{array}{l}\text { Width } \\
{[\mathrm{min}]}\end{array}$ & $\begin{array}{l}\text { Area } \\
{[\mathrm{mAU} * \mathrm{~S}]}\end{array}$ & $\begin{array}{c}\text { Area } \\
\%\end{array}$ \\
\hline 1 & 12.80 & 0.2354 & $1.36279 \mathrm{e} 4$ & 50.6727 \\
2 & 14.85 & 0.2629 & $1.32660 \mathrm{e} 4$ & 49.3273 \\
\hline
\end{tabular}

\begin{tabular}{lllll}
\hline $\begin{array}{l}\text { Peak } \\
\#\end{array}$ & $\begin{array}{l}\text { PetTime } \\
{[\mathrm{min}]}\end{array}$ & $\begin{array}{l}\text { Width } \\
{[\mathrm{min}]}\end{array}$ & $\begin{array}{l}\text { Area } \\
{\left[\mathrm{mAU}^{*} \mathrm{~S}\right]}\end{array}$ & $\begin{array}{c}\text { Area } \\
\%\end{array}$ \\
\hline 1 & 12.67 & 0.2528 & $4.26710 \mathrm{e} 4$ & 97.0140 \\
2 & 14.65 & 0.2414 & 1313.38611 & 2.9860 \\
\hline
\end{tabular}

\section{(2S,3R)-2-(4-isobutylphenyl)-3-methyl-4-phenylpent-4-en-2-ol}

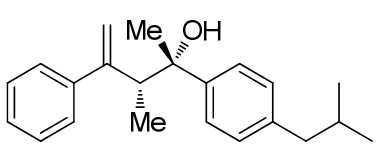

3i

Prepared according to general procedure. In a nitrogen-filled glovebox, a flame-dried screw-cap reaction tube equipped with a Teflon-coated magnetic stir bar were charged with $\mathrm{Cu}(\mathrm{OAc})_{2}(1.8 \mathrm{mg}, 10.0 \mu \mathrm{mol}),(R, R)-\mathrm{Ph}-\mathrm{BPE}$ $(6.1 \mathrm{mg}, 12.0 \mu \mathrm{mol})$, and anhydrous THF $(1 \mathrm{~mL})$, dimethoxymethylsilane (DMMS, 3 equiv) were subsequently added. The resulting mixture was stirred for $2 \mathrm{~min}$ and then buta-1,3-dien-2-ylbenzene $(52.0 \mathrm{mg}, 0.4 \mathrm{mmol})$ and 1-(4-isobutylphenyl) ethanone ( $35.2 \mathrm{mg}, 0.2 \mathrm{mmol}$ ) were added, respectively. The vial was removed from the glove box, and the mixture was stirred at $20{ }^{\circ} \mathrm{C}$ for $18 \mathrm{~h}$. After $18 \mathrm{~h}$ the cap was removed. The solution was quenched by adding saturated ammonium fluoride in methanol using a $5 \mathrm{~mL}$ syringe $(3 \mathrm{~mL})$ and stirred for $30 \mathrm{~min}$. After removal of solvent under reduced pressure with aid of a rotary evaporator, the diastereomeric ratio (dr) was assessed by ${ }^{1} \mathrm{H} \mathrm{NMR}$ in $\mathrm{CDCl}_{3}$, The resulting crude product was purified by flash column chromatography on silica gel (diethyl ether /petroleum ether, 1:10) to obtain product in 95\% (58.5 $\mathrm{mg}$ ) 
yield as a colorless oil. ${ }^{1} \mathbf{H}$ NMR $\left(600 \mathrm{MHz}, \mathrm{CDCl}_{3}\right) \delta 7.40(\mathrm{~d}, J=7.2 \mathrm{~Hz}, 2 \mathrm{H}), 7.34-7.31(\mathrm{~m}, 4 \mathrm{H}), 7.27$ $-7.25(\mathrm{~m}, 1 \mathrm{H}), 7.07$ (d, $J=7.8 \mathrm{~Hz}, 2 \mathrm{H}), 5.43(\mathrm{~s}, 1 \mathrm{H}), 5.26(\mathrm{~s}, 1 \mathrm{H}), 3.23$ (q, $J=7.2 \mathrm{~Hz}, 1 \mathrm{H}), 2.44$ (d, $J=$ $7.2 \mathrm{~Hz}, 2 \mathrm{H}), 1.98(\mathrm{~s}, 1 \mathrm{H}), 1.86-1.82(\mathrm{~m}, 1 \mathrm{H}), 1.32(\mathrm{~s}, 3 \mathrm{H}), 1.02(\mathrm{~d}, J=7.2 \mathrm{~Hz}, 3 \mathrm{H}), 0.89$ (d, $J=6.6 \mathrm{~Hz}$, 6H). ${ }^{13} \mathbf{C}$ NMR $\left(150 \mathrm{MHz}, \mathrm{CDCl}_{3}\right) \delta 152.1,144.9,144.4,139.6,128.6,128.4,127.2,126.4,124.6,115.3$, 76.1, 48.3, 45.0, 30.5, 30.2, 22.4, 16.5. HRMS (ESI-TOF) $(\mathrm{m} / \mathrm{z})$ : Calcd for $\mathrm{C}_{22} \mathrm{H}_{28} \mathrm{ONa}\left([\mathrm{M}+\mathrm{Na}]^{+}\right)$: 331.2032, found: 331.2023 . Specific rotation $[\alpha]^{14} \mathrm{D}=-31.3,\left(\mathrm{c}=1.00, \mathrm{CHCl}_{3}\right)$. HPLC analysis (IA, Hexane $/ \mathrm{IPA}=98 / 2,0.5 \mathrm{~mL} / \mathrm{min}, 250 \mathrm{~nm}$ ) indicated $92 \%$ ee: $\mathrm{tR}$ (major) $=16.15 \mathrm{~min}, \mathrm{tR}($ minor $)=17.45$ $\min$.
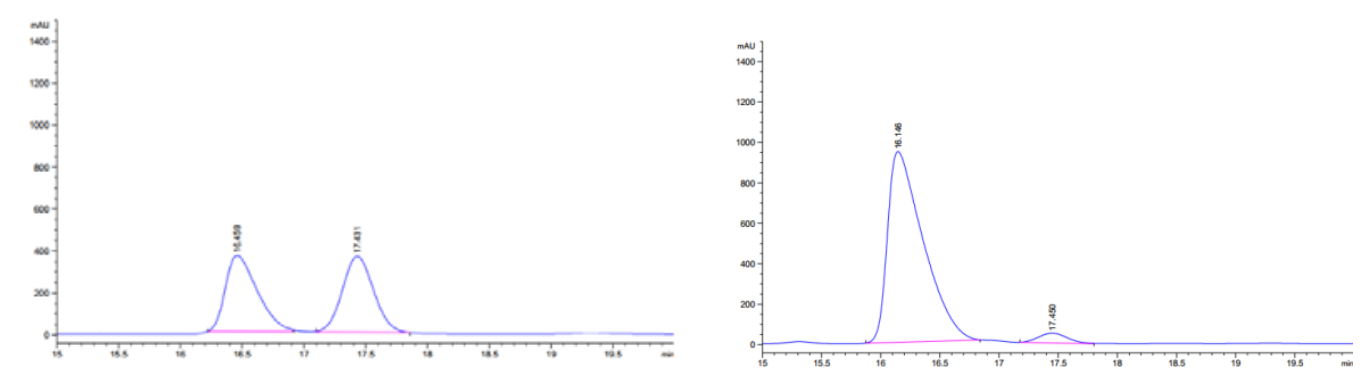

\begin{tabular}{llllc}
\hline $\begin{array}{l}\text { Peak } \\
\#\end{array}$ & $\begin{array}{l}\text { PetTime } \\
{[\mathrm{min}]}\end{array}$ & $\begin{array}{l}\text { Width } \\
{[\mathrm{min}]}\end{array}$ & $\begin{array}{l}\text { Area } \\
{[\mathrm{mAU} * \mathrm{~S}]}\end{array}$ & $\begin{array}{c}\text { Area } \\
\%\end{array}$ \\
\hline 1 & 16.46 & 0.2970 & 6446.52051 & 50.3370 \\
2 & 17.43 & 0.2628 & 6360.21094 & 49.6630 \\
\hline
\end{tabular}

\begin{tabular}{lllll}
\hline $\begin{array}{l}\text { Peak } \\
\#\end{array}$ & $\begin{array}{l}\text { PetTime } \\
{[\mathrm{min}]}\end{array}$ & $\begin{array}{l}\text { Width } \\
{[\mathrm{min}]}\end{array}$ & $\begin{array}{l}\text { Area } \\
{[\mathrm{mAU} * \mathrm{~S}]}\end{array}$ & $\begin{array}{c}\text { Area } \\
\%\end{array}$ \\
\hline 1 & 16.15 & 0.3484 & $1.97908 \mathrm{e} 4$ & 95.9939 \\
2 & 17.45 & 0.2824 & 825.92670 & 4.0061 \\
\hline
\end{tabular}

\section{(2S,3R)-2-([1,1'-biphenyl]-4-yl)-3-methyl-4-phenylpent-4-en-2-ol}

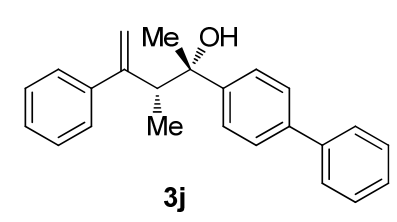

3j

Prepared according to general procedure. In a nitrogen-filled glovebox, a flame-dried screw-cap reaction tube equipped with a Teflon-coated magnetic stir bar were charged with $\mathrm{Cu}(\mathrm{OAc})_{2}(1.8 \mathrm{mg}, 10.0 \mu \mathrm{mol}),(R, R)-$ Ph-BPE $(6.1 \mathrm{mg}, \quad 12.0 \mu \mathrm{mol})$, and anhydrous THF $(1 \mathrm{~mL})$, dimethoxymethylsilane (DMMS, 3 equiv) were subsequently added. The resulting mixture was stirred for $2 \mathrm{~min}$ and then buta-1,3-dien-2-ylbenzene $(52.0 \mathrm{mg}, 0.4 \mathrm{mmol})$ and 1([1,1'-biphenyl]-4-yl) ethanone (39.2 $\mathrm{mg}, 0.2 \mathrm{mmol})$ were added, respectively. The vial was removed from the glove box, and the mixture was stirred at $20{ }^{\circ} \mathrm{C}$ for $18 \mathrm{~h}$. After $18 \mathrm{~h}$ the cap was removed. The solution was quenched by adding saturated ammonium fluoride in methanol using a $5 \mathrm{~mL}$ syringe $(3 \mathrm{~mL})$ and stirred for $30 \mathrm{~min}$. After removal of solvent under reduced pressure with aid of a rotary evaporator, the diastereomeric ratio (dr) was assessed by ${ }^{1} \mathrm{H} \mathrm{NMR} \mathrm{in} \mathrm{CDCl}_{3}$, The resulting crude product was purified by flash column chromatography on silica gel (diethyl ether /petroleum ether, 1:10) to obtain product in $81 \%(53.2 \mathrm{mg})$ yield as a white solid. m.p. $70-71^{\circ} \mathrm{C} .{ }^{1} \mathbf{H}$ NMR $\left(600 \mathrm{MHz}, \mathrm{CDCl}_{3}\right) \delta 7.59(\mathrm{~d}, J=7.2$ $\mathrm{Hz}, 2 \mathrm{H}), 7.54(\mathrm{~d}, J=8.4 \mathrm{~Hz}, 2 \mathrm{H}), 7.49(\mathrm{~d}, J=8.4 \mathrm{~Hz}, 2 \mathrm{H}), 7.44-7.42(\mathrm{~m}, 4 \mathrm{H}), 7.36-7.32(\mathrm{~m}, 3 \mathrm{H})$, $7.29-7.27(\mathrm{~m}, 1 \mathrm{H}), 5.47(\mathrm{~s}, 1 \mathrm{H}), 5.30(\mathrm{~s}, 1 \mathrm{H}), 3.30(\mathrm{q}, J=7.2 \mathrm{~Hz}, 1 \mathrm{H}), 2.05(\mathrm{~s}, 1 \mathrm{H}), 1.36(\mathrm{~s}, 3 \mathrm{H}), 1.07$ $(\mathrm{d}, J=7.2 \mathrm{~Hz}, 3 \mathrm{H}) .{ }^{13} \mathbf{C}$ NMR $\left(150 \mathrm{MHz}, \mathrm{CDCl}_{3}\right) \delta 152.0,146.2,144.8,140.9,139.1,128.7,128.5$, 127.3, 127.1, 127.0, 126.6, 126.4, 125.4, 115.4, 76.1, 48.2, 30.6, 16.5. HRMS (ESI-TOF) (m/z): Calcd for $\mathrm{C}_{24} \mathrm{H}_{24} \mathrm{ONa}\left([\mathrm{M}+\mathrm{Na}]^{+}\right): 351.1719$; found: 351.1718 . Specific rotation $[\alpha]^{14} \mathrm{D}=-30.5,(\mathrm{c}=1.00$, $\mathrm{CHCl}_{3}$ ). HPLC analysis (IA, Hexane/IPA $=98 / 2,0.5 \mathrm{~mL} / \mathrm{min}, 250 \mathrm{~nm}$ ) indicated $92 \%$ ee: $\mathrm{tR}$ (major) $=$ $30.48 \mathrm{~min}, \mathrm{tR}($ minor $)=28.47 \mathrm{~min}$. 

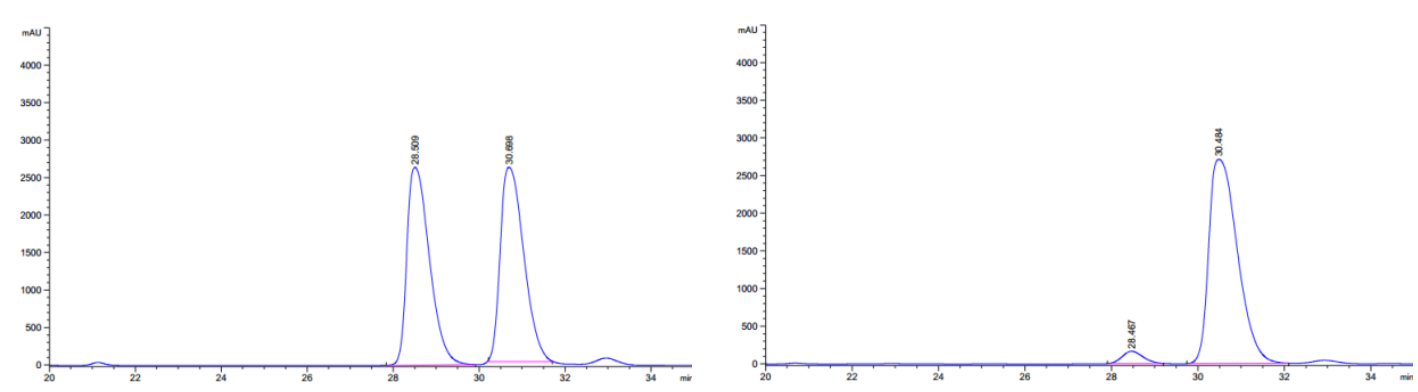

\begin{tabular}{llllc}
\hline $\begin{array}{l}\text { Peak } \\
\#\end{array}$ & $\begin{array}{l}\text { PetTime } \\
{[\mathrm{min}]}\end{array}$ & $\begin{array}{l}\text { Width } \\
{[\mathrm{min}]}\end{array}$ & $\begin{array}{l}\text { Area } \\
{[\mathrm{mAU} * \mathrm{~S}]}\end{array}$ & $\begin{array}{c}\text { Area } \\
\%\end{array}$ \\
\hline 1 & 28.56 & 0.6321 & $9.61956 \mathrm{e} 4$ & 49.4324 \\
2 & 30.70 & 0.2629 & $9.84049 \mathrm{e} 4$ & 50.5679 \\
\hline
\end{tabular}

\begin{tabular}{lllll}
\hline $\begin{array}{l}\text { Peak } \\
\#\end{array}$ & $\begin{array}{l}\text { PetTime } \\
{[\mathrm{min}]}\end{array}$ & $\begin{array}{l}\text { Width } \\
{[\mathrm{min}]}\end{array}$ & $\begin{array}{l}\text { Area } \\
{\left[\mathrm{mAU}^{*} \mathrm{~S}\right]}\end{array}$ & $\begin{array}{c}\text { Area } \\
\%\end{array}$ \\
\hline 1 & 28.47 & 0.5540 & 5383.91504 & 4.1447 \\
2 & 30.48 & 0.7656 & $1.24514 \mathrm{e} 5$ & 95.8553 \\
\hline
\end{tabular}

\section{(2S,3R)-3-methyl-2-(naphthalen-2-yl)-4-phenylpent-4-en-2-ol}

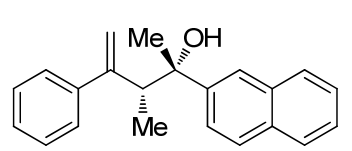

$3 \mathbf{k}$

Prepared according to general procedure. In a nitrogen-filled glovebox, a flame-dried screw-cap reaction tube equipped with a Teflon-coated magnetic stir bar were charged with $\mathrm{Cu}(\mathrm{OAc})_{2}(1.8 \mathrm{mg}, 10.0 \mu \mathrm{mol}),(R, R)$ Ph-BPE (6.1 mg, $12.0 \mu \mathrm{mol})$, and anhydrous THF (1 $\mathrm{mL})$, dimethoxymethylsilane (DMMS, 3 equiv) were subsequently added. The resulting mixture was stirred for $2 \mathrm{~min}$ and then buta-1,3-dien-2-ylbenzene (52.0 $\mathrm{mg}, 0.4 \mathrm{mmol}$ ) and 1(naphthalen-2-yl) ethanone (34.0 mg, $0.2 \mathrm{mmol}$ ) were added, respectively. The vial was removed from the glove box, and the mixture was stirred at $20{ }^{\circ} \mathrm{C}$ for $18 \mathrm{~h}$. After $18 \mathrm{~h}$ the cap was removed. The solution was quenched by adding saturated ammonium fluoride in methanol using a $5 \mathrm{~mL}$ syringe $(3 \mathrm{~mL})$ and stirred for $30 \mathrm{~min}$. After removal of solvent under reduced pressure with aid of a rotary evaporator, the diastereomeric ratio (dr) was assessed by ${ }^{1} \mathrm{H} \mathrm{NMR}$ in $\mathrm{CDCl}_{3}$, The resulting crude product was purified by flash column chromatography on silica gel (diethyl ether /petroleum ether, 1:10) to obtain product in 93\% (56.2 mg) yield as a colorless oil. ${ }^{1} \mathbf{H}$ NMR (600 MHz, $\left.\mathrm{CDCl}_{3}\right) \delta 7.96(\mathrm{~s}, 1 \mathrm{H}), 7.84(\mathrm{~d}, J=7.8 \mathrm{~Hz}$, 1H), 7.79 (t, J=9.6 Hz, 2H), $7.50-7.49(\mathrm{~m}, 1 \mathrm{H}), 7.47-7.42(\mathrm{~m}, 4 \mathrm{H}), 7.35(\mathrm{t}, J=7.2 \mathrm{~Hz}, 2 \mathrm{H}), 7.27$ (t, $J=7.2 \mathrm{~Hz}, 1 \mathrm{H}), 5.47(\mathrm{~s}, 1 \mathrm{H}), 5.32(\mathrm{~s}, 1 \mathrm{H}), 3.39$ (q, $J=7.2 \mathrm{~Hz}, 1 \mathrm{H}), 2.15(\mathrm{~s}, 1 \mathrm{H}), 1.38(\mathrm{~s}, 3 \mathrm{H}), 1.02(\mathrm{~d}, J$ $=7.2 \mathrm{~Hz}, 3 \mathrm{H}) .{ }^{13} \mathbf{C}$ NMR $\left(150 \mathrm{MHz}, \mathrm{CDCl}_{3}\right) \delta 151.94,144.8,144.5,133.1,132.1,128.5,128.2,127.6$, 127.4, 127.3, 126.4, 125.9, 125.5, 123.5, 123.4, 76.2, 47.9, 30.8, 16.5. HRMS (ESI-TOF) (m/z): Calcd for $\mathrm{C}_{22} \mathrm{H}_{22} \mathrm{ONa}\left([\mathrm{M}+\mathrm{Na}]^{+}\right): 325.1563$; found: 325.1570 . Specific rotation $[\alpha]^{14}{ }_{D}=-47.3,(\mathrm{c}=1.00$, $\mathrm{CHCl}_{3}$ ). HPLC analysis (IA, Hexane/IPA = 98/2, $0.5 \mathrm{~mL} / \mathrm{min}, 250 \mathrm{~nm}$ ) indicated 92\% ee: $\mathrm{tR}$ (major) $=$ $16.93 \mathrm{~min}, \mathrm{tR}$ (minor) $=22.28 \mathrm{~min}$.
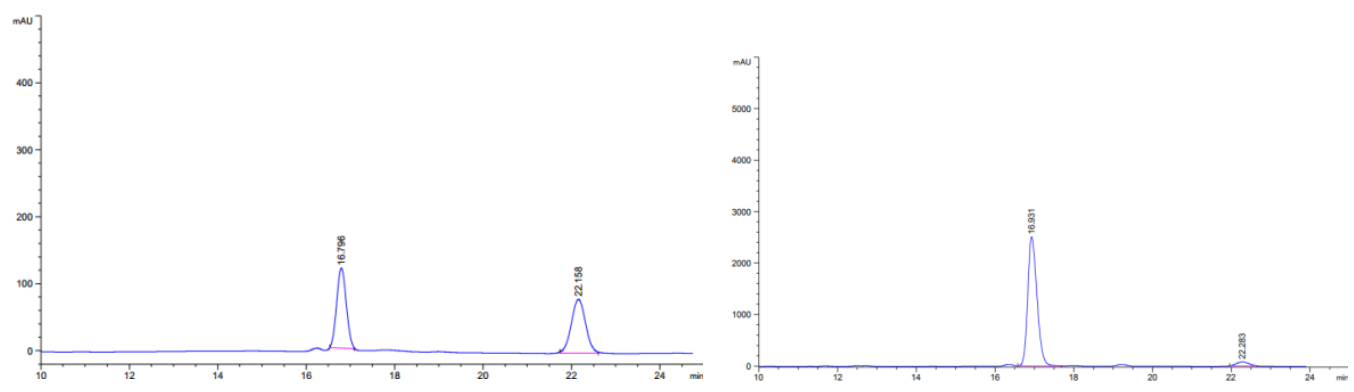


\begin{tabular}{lllll}
\hline $\begin{array}{l}\text { Peak } \\
\#\end{array}$ & $\begin{array}{l}\text { PetTime } \\
{[\mathrm{min}]}\end{array}$ & $\begin{array}{c}\text { Width } \\
{[\mathrm{min}]}\end{array}$ & $\begin{array}{l}\text { Area } \\
{[\mathrm{mAU} * \mathrm{~S}]}\end{array}$ & $\begin{array}{c}\text { Area } \\
\%\end{array}$ \\
\hline 1 & 16.80 & 0.2354 & $1.36279 \mathrm{e} 4$ & 50.6727 \\
2 & 22.16 & 0.2629 & $1.32660 \mathrm{e} 4$ & 49.3273 \\
\hline
\end{tabular}

\begin{tabular}{lllll}
\hline $\begin{array}{l}\text { Peak } \\
\#\end{array}$ & $\begin{array}{l}\text { PetTime } \\
{[\mathrm{min}]}\end{array}$ & $\begin{array}{l}\text { Width } \\
{[\mathrm{min}]}\end{array}$ & $\begin{array}{l}\text { Area } \\
{[\mathrm{mAU} * \mathrm{~S}]}\end{array}$ & $\begin{array}{c}\text { Area } \\
\%\end{array}$ \\
\hline 1 & 16.93 & 0.2728 & $4.10508 \mathrm{e} 4$ & 96.1525 \\
2 & 22.28 & 0.3576 & 1642.65039 & 3.8475 \\
\hline
\end{tabular}

\section{(2S,3R)-2-(4-chlorophenyl)-3-methyl-4-phenylpent-4-en-2-ol}<smiles>C=C(c1ccccc1)[C@H](C)[C@](C)(O)c1ccc(Cl)cc1</smiles>

3I

Prepared according to general procedure. In a nitrogen-filled glovebox, a flame-dried screw-cap reaction tube equipped with a Teflon-coated magnetic stir bar were charged with $\mathrm{Cu}(\mathrm{OAc})_{2}(1.8 \mathrm{mg}, 10.0 \mu \mathrm{mol}),(R, R)-$ Ph-BPE (6.1 mg, $12.0 \mu \mathrm{mol})$, and anhydrous THF $(1 \mathrm{~mL})$, dimethoxymethylsilane (DMMS, 3 equiv) were subsequently added. The resulting mixture was stirred for $2 \mathrm{~min}$ and then buta-1,3-dien-2-ylbenzene (52.0 $\mathrm{mg}, 0.4 \mathrm{mmol}$ ) and 1(4-chlorophenyl) ethanone $(30.9 \mathrm{mg}, 0.2 \mathrm{mmol}$ ) were added, respectively. The vial was removed from the glove box, and the mixture was stirred at $20{ }^{\circ} \mathrm{C}$ for $18 \mathrm{~h}$. After $18 \mathrm{~h}$ the cap was removed. The solution was quenched by adding saturated ammonium fluoride in methanol using a $5 \mathrm{~mL}$ syringe $(3 \mathrm{~mL})$ and stirred for $30 \mathrm{~min}$. After removal of solvent under reduced pressure with aid of a rotary evaporator, the diastereomeric ratio (dr) was assessed by ${ }^{1} \mathrm{H} \mathrm{NMR} \mathrm{in} \mathrm{CDCl}_{3}$, The resulting crude product was purified by flash column chromatography on silica gel (diethyl ether /petroleum ether, 1:10) to obtain product in $77 \%$ (44.1 mg) yield as a colorless oil. ${ }^{1} \mathbf{H}$ NMR (600 MHz, $\left.\mathrm{CDCl}_{3}\right) \delta 7.24(\mathrm{~d}, J=7.8 \mathrm{~Hz}, 2 \mathrm{H}), 7.17-$ $7.21(\mathrm{~m}, 4 \mathrm{H}), 7.13-7.10(\mathrm{~m}, 3 \mathrm{H}), 5.29(\mathrm{~s}, 1 \mathrm{H}), 5.09$ (s, 1H), 3.06 (q, J= $7.2 \mathrm{~Hz}, 1 \mathrm{H}), 1.86(\mathrm{~s}, 1 \mathrm{H}), 1.12$ $(\mathrm{s}, 3 \mathrm{H}), 0.84(\mathrm{~d}, J=7.2 \mathrm{~Hz}, 3 \mathrm{H}) .{ }^{13} \mathbf{C} \mathbf{N M R}\left(150 \mathrm{MHz}, \mathrm{CDCl}_{3}\right) \delta 151.7,145.6,144.6,132.0,128.5,128.0$, 127.3, 126.5, 126.3, 115.5, 75.8, 48.1, 30.6, 16.3. HRMS (ESI-TOF) $(\mathrm{m} / \mathrm{z})$ : Calcd for $\mathrm{C}_{18} \mathrm{H}_{19} \mathrm{ClONa}$ ([M $\left.+\mathrm{Na}]^{+}\right): 309.1025$; found: 309.1022 . Specific rotation $[\alpha]^{14}=-35.7,\left(\mathrm{c}=1.00, \mathrm{CHCl}_{3}\right)$. HPLC analysis (IA, Hexane/IPA = 98/2, $0.5 \mathrm{~mL} / \mathrm{min}, 250 \mathrm{~nm}$ ) indicated $91 \%$ ee: tR (major) $=18.10 \mathrm{~min}$, tR (minor) $=22.92 \mathrm{~min}$.
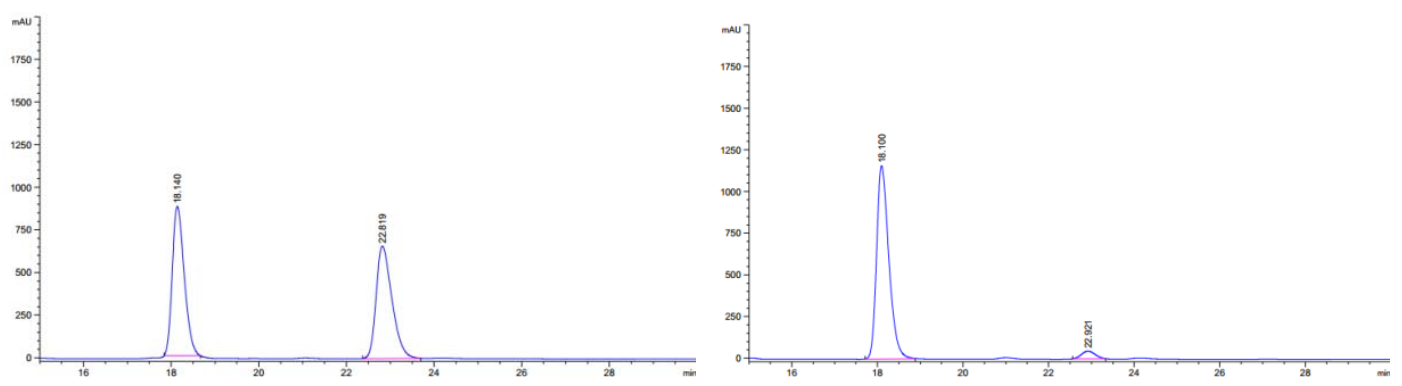

\begin{tabular}{llllc}
\hline $\begin{array}{l}\text { Peak } \\
\#\end{array}$ & $\begin{array}{l}\text { PetTime } \\
{[\mathrm{min}]}\end{array}$ & $\begin{array}{c}\text { Width } \\
{[\mathrm{min}]}\end{array}$ & $\begin{array}{l}\text { Area } \\
{[\mathrm{mAU} * \mathrm{~S}]}\end{array}$ & $\begin{array}{c}\text { Area } \\
\%\end{array}$ \\
\hline 1 & 18.14 & 0.3175 & $1.66834 \mathrm{e} 4$ & 50.2500 \\
2 & 22.82 & 0.4177 & $1.65174 \mathrm{e} 4$ & 49.7500 \\
\hline
\end{tabular}

\begin{tabular}{lllll}
\hline $\begin{array}{l}\text { Peak } \\
\#\end{array}$ & $\begin{array}{l}\text { PetTime } \\
{[\mathrm{min}]}\end{array}$ & $\begin{array}{l}\text { Width } \\
{[\mathrm{min}]}\end{array}$ & $\begin{array}{l}\text { Area } \\
{[\mathrm{mAU} * \mathrm{~S}]}\end{array}$ & $\begin{array}{c}\text { Area } \\
\%\end{array}$ \\
\hline 1 & 18.10 & 0.3313 & $2.30457 \mathrm{e} 4$ & 95.6891 \\
2 & 22.92 & 0.3710 & 1038.22607 & 4.3109 \\
\hline
\end{tabular}




\section{(2S,3R)-2-(3-chlorophenyl)-3-methyl-4-phenylpent-4-en-2-ol}

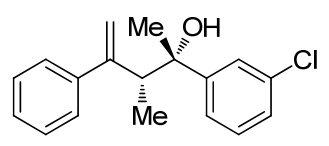

$3 m$

Prepared according to general procedure. In a nitrogen-filled glovebox, a flame-dried screw-cap reaction tube equipped with a Teflon-coated magnetic stir bar were charged with $\mathrm{Cu}(\mathrm{OAc})_{2}(1.8 \mathrm{mg}, 10.0 \mu \mathrm{mol}),(R, R)-\mathrm{Ph}-\mathrm{BPE}(6.1$ $\mathrm{mg}, 12.0 \mu \mathrm{mol})$, and anhydrous THF (1 mL), dimethoxymethylsilane (DMMS, 3 equiv) were subsequently added. The resulting mixture was stirred for $2 \mathrm{~min}$ and then buta-1,3-dien-2-ylbenzene $(52.0 \mathrm{mg}, 0.4 \mathrm{mmol})$ and 1-(3-chlorophenyl) ethanone (30.9 mg, $0.2 \mathrm{mmol}$ ) were added, respectively. The vial was removed from the glove box, and the mixture was stirred at $20{ }^{\circ} \mathrm{C}$ for $18 \mathrm{~h}$. After $18 \mathrm{~h}$ the cap was removed. The solution was quenched by adding saturated ammonium fluoride in methanol using a $5 \mathrm{~mL}$ syringe $(3 \mathrm{~mL})$ and stirred for $30 \mathrm{~min}$. After removal of solvent under reduced pressure with aid of a rotary evaporator, the diastereomeric ratio (dr) was assessed by ${ }^{1} \mathrm{H} \mathrm{NMR}$ in $\mathrm{CDCl}_{3}$, The resulting crude product was purified by flash column chromatography on silica gel (diethyl ether/petroleum ether, 1:10) to obtain product in $76 \%(43.5 \mathrm{mg})$ yield as a colorless oil. ${ }^{1} \mathbf{H}$ NMR $\left(600 \mathrm{MHz}, \mathrm{CDCl}_{3}\right) \delta 7.45-7.44(\mathrm{~m}, 1 \mathrm{H}), 7.41-7.40(\mathrm{~m}, 2 \mathrm{H}), 7.35(\mathrm{t}$, $J=7.8 \mathrm{~Hz}, 2 \mathrm{H}), 7.30-7.28(\mathrm{~m}, 2 \mathrm{H}), 7.24-7.21(\mathrm{~m}, 1 \mathrm{H}), 7.19-7.17(\mathrm{~m}, 1 \mathrm{H}), 5.46(\mathrm{~s}, 1 \mathrm{H}), 5.27(\mathrm{~s}, 1 \mathrm{H})$, $4.23(\mathrm{q}, J=7.2 \mathrm{~Hz}, 1 \mathrm{H}), 1.29(\mathrm{~s}, 1 \mathrm{H}), 1.43(\mathrm{~s}, 3 \mathrm{H}), 1.01$ (d, $J=7.2 \mathrm{~Hz}, 3 \mathrm{H}) .{ }^{13} \mathbf{C} \mathbf{N M R}\left(150 \mathrm{MHz}, \mathrm{CDCl}_{3}\right)$ $\delta 151.7,149.3$ 144.6, 134.0, 129.2, 128.5, 127.4, 126.4, 126.3, 125.4, 123.1, 115.6, 75.8, 48.1, 30.5, 16.3. HRMS (ESI-TOF) $(\mathrm{m} / \mathrm{z})$ : Calcd for $\mathrm{C}_{18} \mathrm{H}_{19} \mathrm{ClONa}\left([\mathrm{M}+\mathrm{Na}]^{+}\right): 309.1025$; found: 309.1027. Specific rotation $[\alpha]^{14} \mathrm{D}=-36.3,\left(\mathrm{c}=1.00, \mathrm{CHCl}_{3}\right)$. $\mathrm{HPLC}$ analysis (IA, Hexane $/ \mathrm{IPA}=98 / 2,0.5 \mathrm{~mL} / \mathrm{min}, 250 \mathrm{~nm}$ ) indicated $92 \%$ ee: $\mathrm{tR}$ (major) $=14.83 \mathrm{~min}$, $\mathrm{tR}($ minor $)=18.27 \mathrm{~min}$.
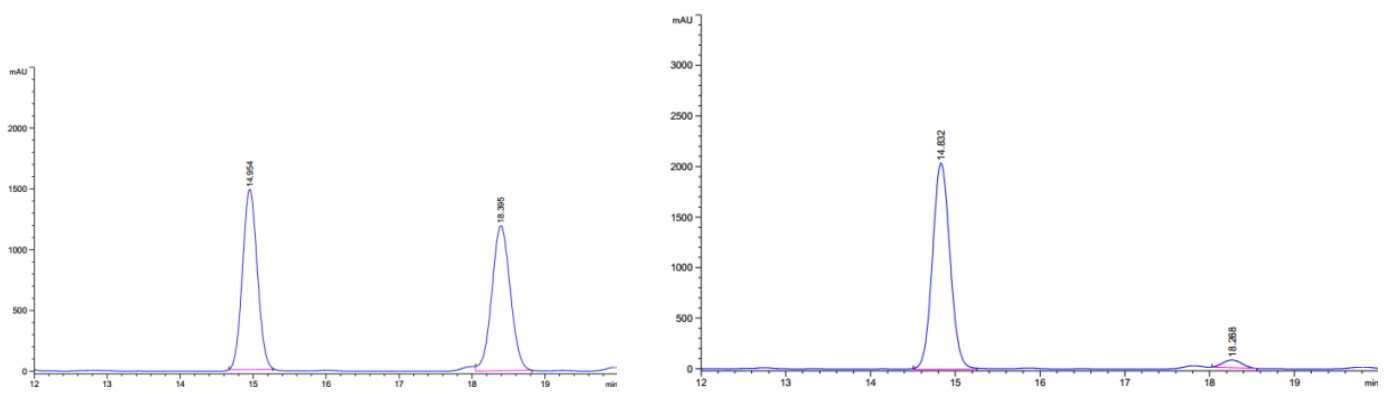

\begin{tabular}{llllc}
\hline $\begin{array}{l}\text { Peak } \\
\#\end{array}$ & $\begin{array}{l}\text { PetTime } \\
{[\mathrm{min}]}\end{array}$ & $\begin{array}{c}\text { Width } \\
{[\mathrm{min}]}\end{array}$ & $\begin{array}{l}\text { Area } \\
{[\mathrm{mAU} * \mathrm{~S}]}\end{array}$ & $\begin{array}{c}\text { Area } \\
\%\end{array}$ \\
\hline 1 & 14.95 & 0.2355 & $2.09176 \mathrm{e} 4$ & 49.9162 \\
2 & 18.40 & 0.2934 & $2.09878 \mathrm{e} 4$ & 50.0838 \\
\hline
\end{tabular}

\begin{tabular}{lllll}
\hline $\begin{array}{l}\text { Peak } \\
\#\end{array}$ & $\begin{array}{l}\text { PetTime } \\
{[\mathrm{min}]}\end{array}$ & $\begin{array}{l}\text { Width } \\
{[\mathrm{min}]}\end{array}$ & $\begin{array}{l}\text { Area } \\
{\left[\mathrm{mAU}{ }^{*} \mathrm{~S}\right]}\end{array}$ & $\begin{array}{c}\text { Area } \\
\%\end{array}$ \\
\hline 1 & 14.83 & 0.2401 & $2.934318 \mathrm{e} 4$ & 96.0937 \\
2 & 18.27 & 0.2571 & 1192.75867 & 3.9063 \\
\hline
\end{tabular}

\section{(2S,3R)-2-(2-chlorophenyl)-3-methyl-4-phenylpent-4-en-2-ol}<smiles>C=C(c1ccccc1)[C@H](C)[C@](C)(O)c1ccccc1Cl</smiles>

3n
Prepared according to general procedure. In a nitrogen-filled glovebox, a flamedried screw-cap reaction tube equipped with a Teflon-coated magnetic stir bar were charged with $\mathrm{Cu}(\mathrm{OAc})_{2}(1.8 \mathrm{mg}, 10.0 \mu \mathrm{mol}),(R, R)-\mathrm{Ph}-\mathrm{BPE}(6.1 \mathrm{mg}, 12.0$ $\mu \mathrm{mol})$, and anhydrous THF ( $1 \mathrm{~mL})$, dimethoxymethylsilane (DMMS, 3 equiv) were subsequently added. The resulting mixture was stirred for $2 \mathrm{~min}$ and then 
buta-1,3-dien-2-ylbenzene $(52.0 \mathrm{mg}, 0.4 \mathrm{mmol})$ and 1-(2-chlorophenyl) ethanone $(30.9 \mathrm{mg}, 0.2 \mathrm{mmol})$ were added, respectively. The vial was removed from the glove box, and the mixture was stirred at $20{ }^{\circ} \mathrm{C}$ for $18 \mathrm{~h}$. After $18 \mathrm{~h}$ the cap was removed. The solution was quenched by adding saturated ammonium fluoride in methanol using a $5 \mathrm{~mL}$ syringe $(3 \mathrm{~mL})$ and stirred for $30 \mathrm{~min}$. After removal of solvent under reduced pressure with aid of a rotary evaporator, the diastereomeric ratio (dr) was assessed by ${ }^{1} \mathrm{H}$ NMR in $\mathrm{CDCl}_{3}$, The resulting crude product was purified by flash column chromatography on silica gel (diethyl ether /petroleum ether, 1:10) to obtain product in $84 \%\left(48.1 \mathrm{mg}\right.$ ) yield as a colorless oil. ${ }^{1} \mathbf{H}$ NMR (600 $\left.\mathrm{MHz}, \mathrm{CDCl}_{3}\right) \delta 7.83(\mathrm{~d}, J=7.8 \mathrm{~Hz}, 1 \mathrm{H}), 7.45(\mathrm{~d}, J=7.2 \mathrm{~Hz}, 2 \mathrm{H}), 7.35-7.32(\mathrm{~m}, 2 \mathrm{H}), 7.29-7.27(\mathrm{~m}$, 2H), 7.23 (d, $J=7.6 \mathrm{~Hz}, 1 \mathrm{H}), 7.14(\mathrm{t}, J=7.2 \mathrm{~Hz}, 1 \mathrm{H}), 5.52(\mathrm{~s}, 1 \mathrm{H}), 5.34$ (s, $1 \mathrm{H}), 4.23$ (q, $J=7.2 \mathrm{~Hz}$, 1H), $2.36(\mathrm{~s}, 1 \mathrm{H}), 1.43$ (s, 3H), 0.98 (d, $J=7.2 \mathrm{~Hz}, 3 \mathrm{H}) .{ }^{13} \mathbf{C} \mathbf{~ N M R}\left(150 \mathrm{MHz}, \mathrm{CDCl}_{3}\right) \delta 152.0,144.2$, 143.4, 131.1, 130.3, 128.5, 128.4, 128.0 127.3, 126.7, 126.4, 115.9, 76.1, 42.5, 27.5, 16.3. HRMS (ESITOF) $(\mathrm{m} / \mathrm{z})$ : Calcd for $\mathrm{C}_{18} \mathrm{H}_{19} \mathrm{ClONa}\left([\mathrm{M}+\mathrm{Na}]^{+}\right): 309.1025$; found: 309.1021 . Specific rotation $[\alpha]^{15} \mathrm{D}$ $=-37.1,\left(\mathrm{c}=1.00, \mathrm{CHCl}_{3}\right)$. HPLC analysis (IA, Hexane/IPA $=98 / 2,0.5 \mathrm{~mL} / \mathrm{min}, 250 \mathrm{~nm}$ ) indicated $92 \%$ ee: $\operatorname{tR}($ major $)=13.08 \mathrm{~min}, \mathrm{tR}($ minor $)=13.94 \mathrm{~min}$.

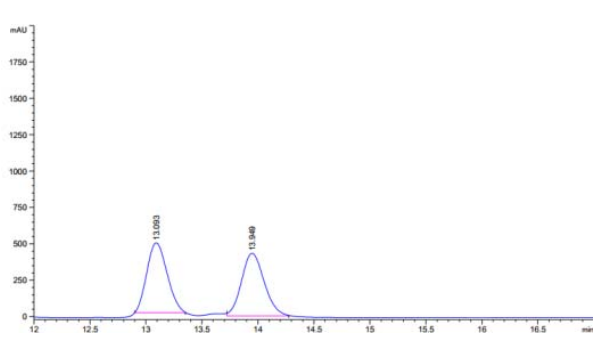

\begin{tabular}{lllll}
\hline $\begin{array}{l}\text { Peak } \\
\#\end{array}$ & $\begin{array}{l}\text { PetTime } \\
{[\mathrm{min}]}\end{array}$ & $\begin{array}{c}\text { Width } \\
{[\mathrm{min}]}\end{array}$ & $\begin{array}{l}\text { Area } \\
{[\mathrm{mAU} * \mathrm{~S}]}\end{array}$ & $\begin{array}{c}\text { Area } \\
\%\end{array}$ \\
\hline 1 & 13.10 & 0.2097 & 6043.52295 & 49.8984 \\
2 & 13.94 & 0.2348 & 6068.13123 & 50.1016 \\
\hline
\end{tabular}

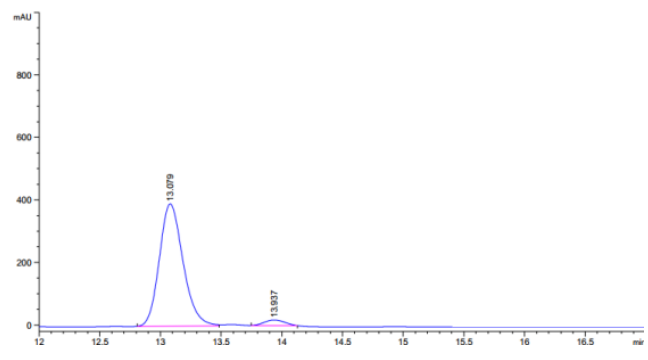

\begin{tabular}{lllll}
\hline $\begin{array}{l}\text { Peak } \\
\#\end{array}$ & $\begin{array}{l}\text { PetTime } \\
{[\mathrm{min}]}\end{array}$ & $\begin{array}{l}\text { Width } \\
{[\mathrm{min}]}\end{array}$ & $\begin{array}{l}\text { Area } \\
{\left[\mathrm{mAU}^{*} \mathrm{~S}\right]}\end{array}$ & $\begin{array}{c}\text { Area } \\
\%\end{array}$ \\
\hline 1 & 13.08 & 0.2267 & 5323.55469 & 96.2174 \\
2 & 13.94 & 0.1946 & 209.28453 & 3.7826 \\
\hline
\end{tabular}

\section{(2S,3R)-2-(3-bromophenyl)-3-methyl-4-phenylpent-4-en-2-ol}<smiles>[M]C(C(=C)c1ccccc1)C(O)(O)c1cccc(Br)c1</smiles>

30

Prepared according to general procedure. In a nitrogen-filled glovebox, a flame-dried screw-cap reaction tube equipped with a Teflon-coated magnetic stir bar were charged with $\mathrm{Cu}(\mathrm{OAc})_{2}(1.8 \mathrm{mg}, 10.0 \mu \mathrm{mol})$, $(R, R)$-Ph-BPE $(6.1 \mathrm{mg}, 12.0 \mu \mathrm{mol})$, and anhydrous THF $(1 \mathrm{~mL})$, dimethoxymethylsilane (DMMS, 3 equiv) were subsequently added.

The resulting mixture was stirred for $2 \mathrm{~min}$ and then buta-1,3-dien-2-ylbenzene $(52.0 \mathrm{mg}, 0.4 \mathrm{mmol}$ ) and 1-(3-bromophenyl) ethanone ( $39.8 \mathrm{mg}, 0.2 \mathrm{mmol}$ ) were added, respectively. The vial was removed from the glove box, and the mixture was stirred at $20{ }^{\circ} \mathrm{C}$ for $18 \mathrm{~h}$. After $18 \mathrm{~h}$ the cap was removed. The solution was quenched by adding saturated ammonium fluoride in methanol using a $5 \mathrm{~mL}$ syringe $(3 \mathrm{~mL})$ and stirred for $30 \mathrm{~min}$. After removal of solvent under reduced pressure with aid of a rotary evaporator, the diastereomeric ratio (dr) was assessed by ${ }^{1} \mathrm{H} N M R$ in $\mathrm{CDCl}_{3}$, The resulting crude product was purified by flash column chromatography on silica gel (diethyl ether /petroleum ether, 1:10) to obtain product in $54 \%(35.7 \mathrm{mg})$ yield as a yellow oil. ${ }^{1} \mathbf{H}$ NMR $\left(600 \mathrm{MHz}, \mathrm{CDCl}_{3}\right) \delta 7.60(\mathrm{~s}, 1 \mathrm{H}), 7.40(\mathrm{~d}, J=7.2 \mathrm{~Hz}$, 2H), $7.36-7.33(\mathrm{~m}, 4 \mathrm{H}), 7.28(\mathrm{t}, J=7.2 \mathrm{~Hz}, 1 \mathrm{H}), 7.17(\mathrm{t}, J=7.8 \mathrm{~Hz}, 1 \mathrm{H}), 5.46(\mathrm{~s}, 1 \mathrm{H}), 5.27(\mathrm{~s}, 1 \mathrm{H})$, $3.22(\mathrm{q}, J=7.2 \mathrm{~Hz}, 1 \mathrm{H}), 2.02(\mathrm{~s}, 1 \mathrm{H}), 1.28(\mathrm{~s}, 3 \mathrm{H}), 1.01(\mathrm{~d}, J=7.2 \mathrm{~Hz}, 3 \mathrm{H}) .{ }^{13} \mathbf{C} \mathbf{N M R}\left(150 \mathrm{MHz}, \mathrm{CDCl}_{3}\right)$ 
$\delta$ 151.7, 149.5, 144.6 129.5, 129.4, 128.5, 128.3, 127.4, 126.3, 123.6, 122.3, 115.6, 75.8, 48.1, 30.5, 16.3 . HRMS (ESI-TOF) $(\mathrm{m} / \mathrm{z})$ : Calcd for $\mathrm{C}_{18} \mathrm{H}_{19} \mathrm{BrONa}\left([\mathrm{M}+\mathrm{Na}]^{+}\right)$: 353.0512; found: 353.0515 . Specific rotation $[\alpha]^{14} \mathrm{D}=-29.3,\left(\mathrm{c}=1.00, \mathrm{CHCl}_{3}\right) . \mathrm{HPLC}$ analysis $(\mathrm{IA}, \mathrm{Hexane} / \mathrm{IPA}=98 / 2,0.5 \mathrm{~mL} / \mathrm{min}, 250 \mathrm{~nm})$ indicated $92 \%$ ee: $\mathrm{tR}$ (major) $=14.79 \mathrm{~min}, \mathrm{tR}($ minor $)=17.87 \mathrm{~min}$.
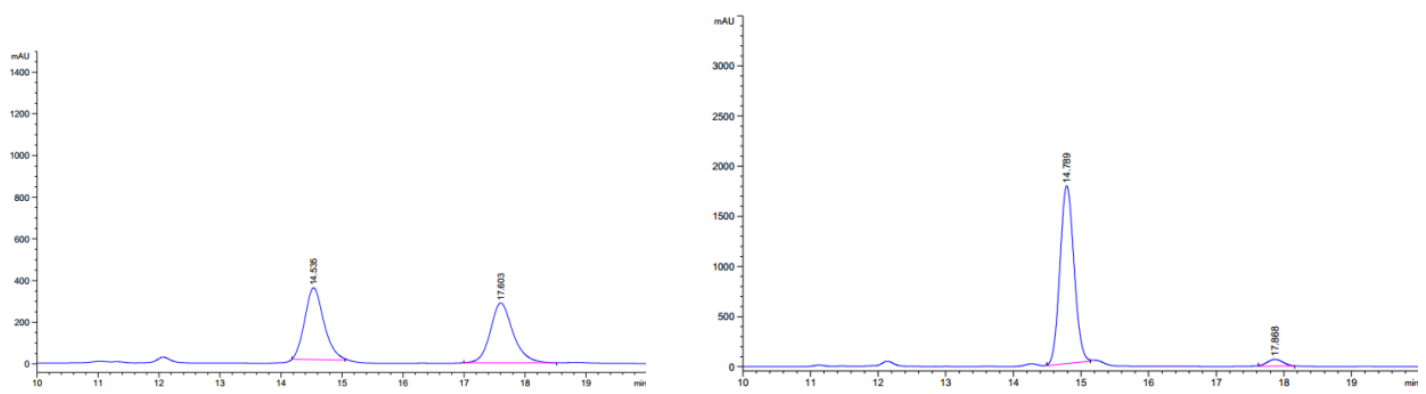

\begin{tabular}{llllc}
\hline $\begin{array}{l}\text { Peak } \\
\#\end{array}$ & $\begin{array}{l}\text { PetTime } \\
{[\mathrm{min}]}\end{array}$ & $\begin{array}{l}\text { Width } \\
{[\mathrm{min}]}\end{array}$ & $\begin{array}{l}\text { Area } \\
{[\mathrm{mAU} * \mathrm{~S}]}\end{array}$ & $\begin{array}{c}\text { Area } \\
\%\end{array}$ \\
\hline 1 & 14.54 & 0.3635 & 7510.75000 & 50.2480 \\
2 & 17.60 & 0.4308 & 7436.60547 & 49.7520 \\
\hline
\end{tabular}

\begin{tabular}{llllr}
\hline $\begin{array}{l}\text { Peak } \\
\#\end{array}$ & $\begin{array}{l}\text { PetTime } \\
{[\mathrm{min}]}\end{array}$ & $\begin{array}{l}\text { Width } \\
{[\mathrm{min}]}\end{array}$ & \multicolumn{1}{l}{$\begin{array}{l}\text { Area } \\
{\left[\mathrm{mAU}^{*} \mathrm{~S}\right]}\end{array}$} & \multicolumn{1}{c}{$\begin{array}{c}\text { Area } \\
\%\end{array}$} \\
\hline 1 & 14.79 & 0.2406 & $2.56166 \mathrm{e} 4$ & 95.8858 \\
2 & 17.87 & 0.2720 & 67.34501 & 4.1142 \\
\hline
\end{tabular}

\section{(2S,3R)-2-(4-iodophenyl)-3-methyl-4-phenylpent-4-en-2-ol}

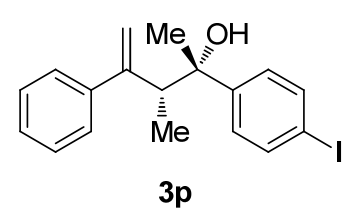

Prepared according to general procedure. In a nitrogen-filled glovebox, a flame-dried screw-cap reaction tube equipped with a Teflon-coated magnetic stir bar were charged with $\mathrm{Cu}(\mathrm{OAc})_{2}(1.8 \mathrm{mg}, 10.0 \mu \mathrm{mol}),(R, R)-\mathrm{Ph}-\mathrm{BPE}(6.1$ $\mathrm{mg}, 12.0 \mu \mathrm{mol})$, and anhydrous THF $(1 \mathrm{~mL})$, dimethoxymethylsilane (DMMS, 3 equiv) were subsequently added. The resulting mixture was stirred for $2 \mathrm{~min}$ and then buta-1,3-dien-2-ylbenzene ( $52.0 \mathrm{mg}, 0.4 \mathrm{mmol})$ and 1-(4-iodophenyl) ethanone ( $49.2 \mathrm{mg}, 0.2 \mathrm{mmol}$ ) were added, respectively. The vial was removed from the glove box, and the mixture was stirred at $20{ }^{\circ} \mathrm{C}$ for $18 \mathrm{~h}$. After $18 \mathrm{~h}$ the cap was removed. The solution was quenched by adding saturated ammonium fluoride in methanol using a $5 \mathrm{~mL}$ syringe $(3 \mathrm{~mL})$ and stirred for $30 \mathrm{~min}$. After removal of solvent under reduced pressure with aid of a rotary evaporator, the diastereomeric ratio (dr) was assessed by ${ }^{1} \mathrm{H}$ NMR in $\mathrm{CDCl}_{3}$, The resulting crude product was purified by flash column chromatography on silica gel (diethyl ether /petroleum ether, 1:10) to obtain product in $59 \%(44.6 \mathrm{mg})$ yield as a yellow oil. ${ }^{1} \mathbf{H}$ NMR $\left(600 \mathrm{MHz}, \mathrm{CDCl}_{3}\right) \delta 7.62(\mathrm{~d}, J=8.4 \mathrm{~Hz}, 2 \mathrm{H}), 7.40(\mathrm{~d}, J=7.6 \mathrm{~Hz}, 2 \mathrm{H})$, $7.35(\mathrm{t}, J=7.5 \mathrm{~Hz}, 2 \mathrm{H}), 7.28(\mathrm{t}, J=7.3 \mathrm{~Hz}, 1 \mathrm{H}), 7.18(\mathrm{~d}, J=8.4 \mathrm{~Hz}, 2 \mathrm{H}), 5.45(\mathrm{~s}, 1 \mathrm{H}), 5.25(\mathrm{~s}, 1 \mathrm{H}), 3.21$ (q, $J=7.2 \mathrm{~Hz}, 1 \mathrm{H}), 1.99(\mathrm{~s}, 1 \mathrm{H}), 1.27(\mathrm{~s}, 3 \mathrm{H}), 1.00(\mathrm{~d}, J=7.2 \mathrm{~Hz}, 3 \mathrm{H}) .{ }^{13} \mathbf{C} \mathbf{N M R}\left(151 \mathrm{MHz}, \mathrm{CDCl}_{3}\right) \delta$ 151.7, 146.8, 144.6, 136.9, 128.5, 127.4, 127.2, 126.3, 115.5, 91.8, 75.9, 48.0, 30.5, 16.3. HRMS (ESITOF) (m/z): $\mathrm{C}_{18} \mathrm{H}_{19} \mathrm{IONa}\left([\mathrm{M}+\mathrm{Na}]^{+}\right): 401.0378$; found: 401.0381 . Specific rotation $[\alpha]^{14} \mathrm{D}=-28.7$, $(\mathrm{c}$ $=1.00, \mathrm{CHCl}_{3}$ ). HPLC analysis (IA, Hexane $/ \mathrm{IPA}=98 / 2,0.5 \mathrm{~mL} / \mathrm{min}, 250 \mathrm{~nm}$ ) indicated $89 \%$ ee: tR $($ major $)=21.75 \mathrm{~min}, \mathrm{tR}($ minor $)=25.36 \mathrm{~min}$. 

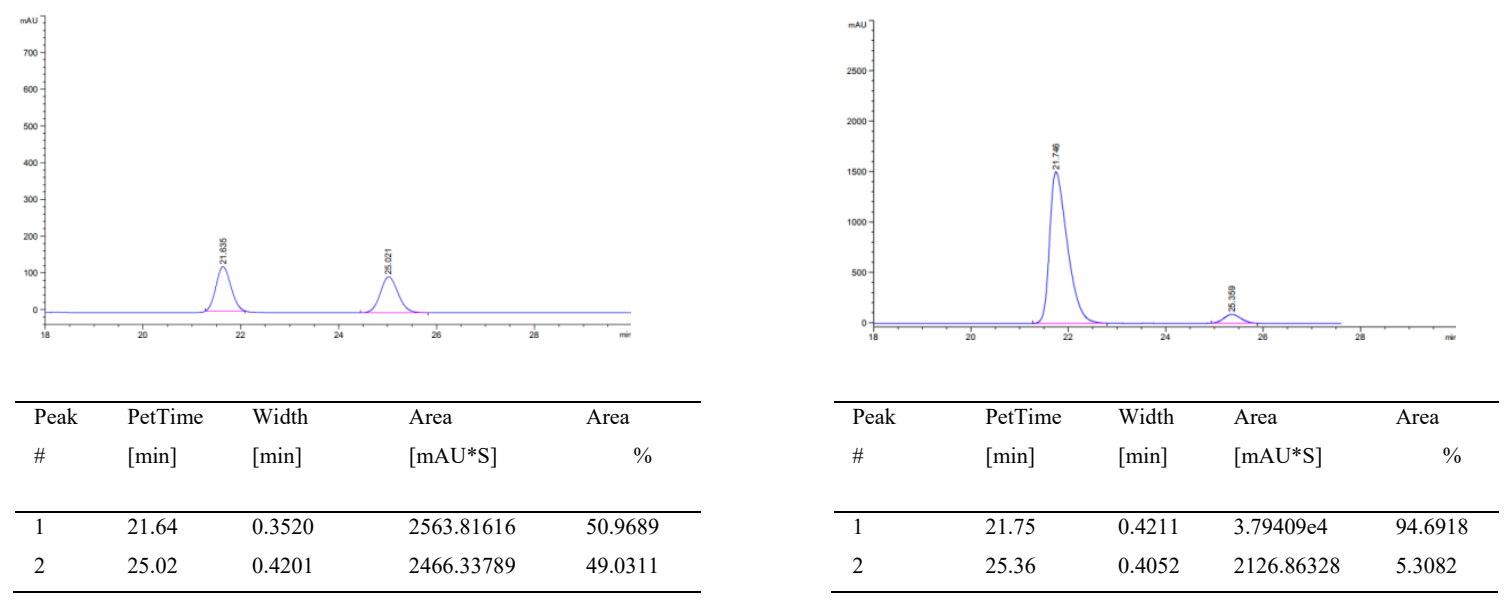

\section{(2S,3R)-3-methyl-4-phenyl-2-(4-(trifluoromethyl)phenyl)pent-4-en-2-ol}<smiles>C=C(c1ccccc1)[C@H](C)[C@](C)(O)c1ccc(C(F)(F)F)cc1</smiles>

$3 q$

Prepared according to general procedure. In a nitrogen-filled glovebox, a flame-dried screw-cap reaction tube equipped with a Teflon-coated magnetic stir bar were charged with $\mathrm{Cu}(\mathrm{OAc})_{2}(1.8 \mathrm{mg}, 10.0 \mu \mathrm{mol}),(R, R)$ Ph-BPE (6.1 mg, $12.0 \mu \mathrm{mol})$, and anhydrous THF $(1 \mathrm{~mL})$, dimethoxymethylsilane (DMMS, 3 equiv) were subsequently added. The resulting mixture was stirred for $2 \mathrm{~min}$ and then buta-1,3-dien-2-ylbenzene (52.0 $\mathrm{mg}, 0.4 \mathrm{mmol})$ and 1(4-(trifluoromethyl)phenyl) ethanone $(37.6 \mathrm{mg}, 0.2 \mathrm{mmol}$ ) were added, respectively. The vial was removed from the glove box, and the mixture was stirred at $20{ }^{\circ} \mathrm{C}$ for $18 \mathrm{~h}$. After $18 \mathrm{~h}$ the cap was removed. The solution was quenched by adding saturated ammonium fluoride in methanol using a $5 \mathrm{~mL}$ syringe $(3 \mathrm{~mL})$ and stirred for $30 \mathrm{~min}$. After removal of solvent under reduced pressure with aid of a rotary evaporator, the diastereomeric ratio (dr) was assessed by ${ }^{1} \mathrm{H} \mathrm{NMR}$ in $\mathrm{CDCl}_{3}$, The resulting crude product was purified by flash column chromatography on silica gel (diethyl ether /petroleum ether, 1:10) to obtain product in $62 \%(40.0 \mathrm{mg})$ yield as a colorless oil. ${ }^{1} \mathbf{H} \mathbf{~ N M R}\left(600 \mathrm{MHz}, \mathrm{CDCl}_{3}\right) \delta 7.56(\mathrm{~s}, 4 \mathrm{H})$, $7.41(\mathrm{~d}, J=7.2 \mathrm{~Hz}, 2 \mathrm{H}), 7.35$ (t, $J=7.8 \mathrm{~Hz}, 2 \mathrm{H}), 7.29$ (t, $J=7.2 \mathrm{~Hz}, 1 \mathrm{H}), 5.47(\mathrm{~s}, 1 \mathrm{H}), 5.28(\mathrm{~s}, 1 \mathrm{H}), 3.27$ $(\mathrm{q}, J=7.2 \mathrm{~Hz}, 1 \mathrm{H}), 2.08(\mathrm{~s}, 1 \mathrm{H}), 1.32(\mathrm{~s}, 3 \mathrm{H}), 1.00(\mathrm{~d}, J=7.2 \mathrm{~Hz}, 3 \mathrm{H}) .{ }^{13} \mathbf{C} \mathbf{N M R}(150 \mathrm{MHz}, \mathrm{CDCl} 3) \delta$ 151.6, 151.2, 144.6, 128.6, 127.4, 126.3, 125.4, 124.9 (q, $J=4.5 \mathrm{~Hz}), 124.3$ (q, $J=262.0 \mathrm{~Hz}), 115.7$, 76.0, 48.1, 30.5, 16.3. ${ }^{19}$ F NMR (470 MHz, $\left.\mathrm{CDCl}_{3}\right) \delta-62.33$ (s, 3F). HRMS (ESI-TOF) (m/z): Calcd for $\mathrm{C}_{19} \mathrm{H}_{19} \mathrm{~F}_{3} \mathrm{ONa}\left([\mathrm{M}+\mathrm{Na}]^{+}\right): 343.1286$; found: 343.1287 . Specific rotation $[\alpha]^{13}{ }_{\mathrm{D}}=-32.5,(\mathrm{c}=1.00$, $\mathrm{CHCl}_{3}$ ). HPLC analysis (IA, Hexane/IPA = 98/2, $0.5 \mathrm{~mL} / \mathrm{min}, 250 \mathrm{~nm}$ ) indicated $90 \%$ ee: $\mathrm{tR}$ (major) = $17.15 \mathrm{~min}, \mathrm{tR}$ (minor) $=19.24 \mathrm{~min}$.
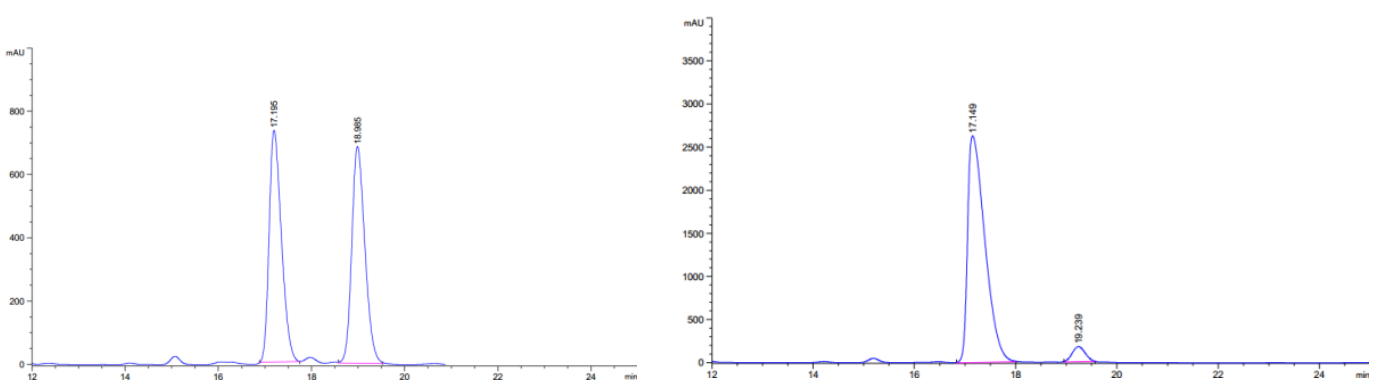


\begin{tabular}{llllc}
\hline $\begin{array}{l}\text { Peak } \\
\#\end{array}$ & $\begin{array}{l}\text { PetTime } \\
{[\mathrm{min}]}\end{array}$ & $\begin{array}{c}\text { Width } \\
{[\mathrm{min}]}\end{array}$ & $\begin{array}{l}\text { Area } \\
{[\mathrm{mAU} * \mathrm{~S}]}\end{array}$ & $\begin{array}{c}\text { Area } \\
\%\end{array}$ \\
\hline 1 & 17.20 & 0.3048 & $1.34102 \mathrm{e} 4$ & 50.0714 \\
2 & 19.00 & 0.3253 & $1.33719 \mathrm{e} 4$ & 49.9286 \\
\hline
\end{tabular}

\begin{tabular}{llllr}
\hline $\begin{array}{l}\text { Peak } \\
\#\end{array}$ & $\begin{array}{l}\text { PetTime } \\
{[\mathrm{min}]}\end{array}$ & $\begin{array}{l}\text { Width } \\
{[\mathrm{min}]}\end{array}$ & $\begin{array}{l}\text { Area } \\
{[\mathrm{mAU} * \mathrm{~S}]}\end{array}$ & \multicolumn{1}{c}{$\begin{array}{c}\text { Area } \\
\%\end{array}$} \\
\hline 1 & 17.15 & 0.3946 & $6.22352 \mathrm{e} 4$ & 95.2274 \\
2 & 19.24 & 0.2963 & 3119.10498 & 4.7726 \\
\hline
\end{tabular}

\section{(2S,3R)-2-(benzo[b]thiophen-2-yl)-3-methyl-4-phenylpent-4-en-2-ol}

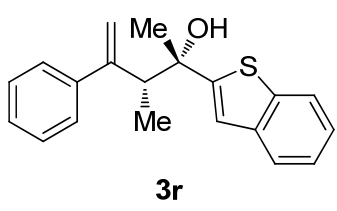

Prepared according to general procedure. In a nitrogen-filled glovebox, a flame-dried screw-cap reaction tube equipped with a Teflon-coated magnetic stir bar were charged with $\mathrm{Cu}(\mathrm{OAc})_{2}(1.8 \mathrm{mg}, 10.0 \mu \mathrm{mol}),(R, R)-\mathrm{Ph}-\mathrm{BPE}(6.1$ $\mathrm{mg}, 12.0 \mu \mathrm{mol})$, and anhydrous THF $(1 \mathrm{~mL})$, dimethoxymethylsilane (DMMS, 3 equiv) were subsequently added. The resulting mixture was stirred for $2 \mathrm{~min}$ and then buta-1,3-dien-2-ylbenzene ( $52.0 \mathrm{mg}, 0.4 \mathrm{mmol}$ ) and 1-(benzo[b]thiophen-2-yl) ethanone (35.2 $\mathrm{mg}, 0.2 \mathrm{mmol}$ ) were added, respectively. The vial was removed from the glove box, and the mixture was stirred at $20{ }^{\circ} \mathrm{C}$ for $18 \mathrm{~h}$. After $18 \mathrm{~h}$ the cap was removed. The solution was quenched by adding saturated ammonium fluoride in methanol using a $5 \mathrm{~mL}$ syringe $(3 \mathrm{~mL})$ and stirred for $30 \mathrm{~min}$. After removal of solvent under reduced pressure with aid of a rotary evaporator, the diastereomeric ratio (dr) was assessed by ${ }^{1} \mathrm{H}$ NMR in $\mathrm{CDCl}_{3}$, The resulting crude product was purified by flash column chromatography on silica gel (diethyl ether /petroleum ether, 1:10) to obtain product in $71 \%(43.7 \mathrm{mg})$ yield as a yellow oil. ${ }^{1} \mathbf{H}$ NMR $\left(600 \mathrm{MHz}, \mathrm{CDCl}_{3}\right) \delta 7.78(\mathrm{~d}, J=8.4 \mathrm{~Hz}, 1 \mathrm{H}), 7.69(\mathrm{~d}, J=8.4 \mathrm{~Hz}, 1 \mathrm{H})$, $7.42(\mathrm{~d}, J=7.2 \mathrm{~Hz}, 2 \mathrm{H}), 7.35-7.30(\mathrm{~m}, 3 \mathrm{H}), 7.28-7.25(\mathrm{~m}, 2 \mathrm{H}), 7.14(\mathrm{~s}, 1 \mathrm{H}), 5.45(\mathrm{~s}, 1 \mathrm{H}), 5.32(\mathrm{~s}$, 1H), 3.32 (q, $J=7.2 \mathrm{~Hz}, 1 \mathrm{H}), 2.25$ (s, $1 \mathrm{H}), 1.48$ (s, $3 \mathrm{H}), 1.23$ (d, $J=7.2 \mathrm{~Hz}, 3 \mathrm{H}) .{ }^{13} \mathbf{C}$ NMR $(150 \mathrm{MHz}$, $\left.\mathrm{CDCl}_{3}\right) \delta 153.4,151.2,144.4,140.0,139.1,128.5,127.4,126.5,124.1,123.7,123.2,122.2,118.9,115.8$, 76.5, 49.1, 31.4, 16.7. HRMS (ESI-TOF) $(\mathrm{m} / \mathrm{z})$ : Calcd for $\mathrm{C}_{20} \mathrm{H}_{20} \mathrm{OSNa}\left([\mathrm{M}+\mathrm{Na}]^{+}\right): 331.1128$; found: 331.1131. Specific rotation $[\alpha]^{15} \mathrm{D}=-40.6,\left(\mathrm{c}=1.00, \mathrm{CHCl}_{3}\right)$. HPLC analysis (IA, Hexane/IPA $=98 / 2$, $0.5 \mathrm{~mL} / \mathrm{min}, 250 \mathrm{~nm}$ ) indicated $96 \%$ ee: $\mathrm{tR}$ (major) $=33.04 \mathrm{~min}, \mathrm{tR}($ minor $)=47.03 \mathrm{~min}$.
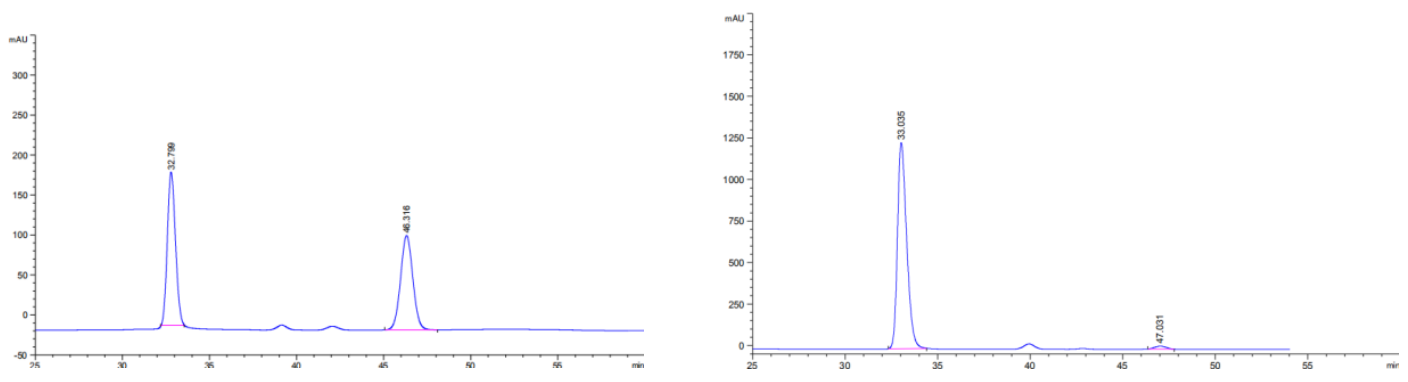

\begin{tabular}{llllc}
\hline $\begin{array}{l}\text { Peak } \\
\#\end{array}$ & $\begin{array}{l}\text { PetTime } \\
{[\mathrm{min}]}\end{array}$ & $\begin{array}{l}\text { Width } \\
{[\mathrm{min}]}\end{array}$ & $\begin{array}{l}\text { Area } \\
{[\mathrm{mAU} * \mathrm{~S}]}\end{array}$ & $\begin{array}{c}\text { Area } \\
\%\end{array}$ \\
\hline 1 & 32.80 & 0.5411 & 6219.44922 & 51.7965 \\
2 & 46.32 & 0.8180 & 5788.02783 & 48.2035 \\
\hline
\end{tabular}

\begin{tabular}{lllll}
\hline $\begin{array}{l}\text { Peak } \\
\#\end{array}$ & $\begin{array}{l}\text { PetTime } \\
{[\mathrm{min}]}\end{array}$ & $\begin{array}{l}\text { Width } \\
{[\mathrm{min}]}\end{array}$ & $\begin{array}{l}\text { Area } \\
{\left[\mathrm{mAU}^{*} \mathrm{~S}\right]}\end{array}$ & $\begin{array}{c}\text { Area } \\
\%\end{array}$ \\
\hline 1 & 33.04 & 0.5751 & $4.28133 \mathrm{e} 4$ & 98.2250 \\
2 & 47.03 & 0.7112 & 773.64783 & 1.7750 \\
\hline
\end{tabular}




\section{(2S,3R)-3-methyl-2-(1-methyl-1H-pyrrol-2-yl)-4-phenylpent-4-en-2-ol}

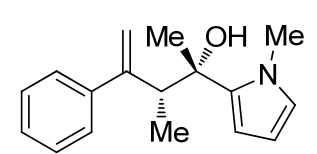

$3 \mathbf{s}$

Prepared according to general procedure. In a nitrogen-filled glovebox, a flamedried screw-cap reaction tube equipped with a Teflon-coated magnetic stir bar were charged with $\mathrm{Cu}(\mathrm{OAc})_{2}(1.8 \mathrm{mg}, 10.0 \mu \mathrm{mol}),(R, R)-\mathrm{Ph}-\mathrm{BPE}(6.1 \mathrm{mg}, 12.0$ $\mu \mathrm{mol})$, and anhydrous THF ( $1 \mathrm{~mL})$, dimethoxymethylsilane (DMMS, 3 equiv) were subsequently added. The resulting mixture was stirred for $2 \mathrm{~min}$ and then buta-1,3-dien-2-ylbenzene (52.0 mg, $0.4 \mathrm{mmol}$ ) and 1-(1-methyl-1H-pyrrol-2-yl) ethanone (24.6 mg, 0.2 $\mathrm{mmol}$ ) were added, respectively. The vial was removed from the glove box, and the mixture was stirred at $20{ }^{\circ} \mathrm{C}$ for $18 \mathrm{~h}$. After $18 \mathrm{~h}$ the cap was removed. The solution was quenched by adding saturated ammonium fluoride in methanol using a $5 \mathrm{~mL}$ syringe $(3 \mathrm{~mL})$ and stirred for $30 \mathrm{~min}$. After removal of solvent under reduced pressure with aid of a rotary evaporator, the diastereomeric ratio (dr) was assessed by ${ }^{1} \mathrm{H}$ NMR in $\mathrm{CDCl}_{3}$, The resulting crude product was purified by flash column chromatography on silica gel (diethyl ether /petroleum ether, 1:10) to obtain product in $86 \%(43.9 \mathrm{mg}$ ) yield as a white solid. m.p. $74-76{ }^{\circ} \mathrm{C} .{ }^{1} \mathbf{H}$ NMR $\left(600 \mathrm{MHz}, \mathrm{CDCl}_{3}\right) \delta 7.21(\mathrm{t}, J=7.4 \mathrm{~Hz}, 2 \mathrm{H}), 7.16(\mathrm{t}, J=7.2 \mathrm{~Hz}, 1 \mathrm{H}), 7.11$ (d, $J=7.2 \mathrm{~Hz}, 2 \mathrm{H}), 6.20(\mathrm{t}, J=2.4 \mathrm{~Hz}, 1 \mathrm{H}), 5.87$ (d, $J=2.4 \mathrm{~Hz}, 2 \mathrm{H}), 5.25(\mathrm{~s}, 1 \mathrm{H}), 5.19(\mathrm{~s}, 1 \mathrm{H}), 3.60(\mathrm{~s}$, 3H), 3.40 (q, $J=7.2 \mathrm{~Hz}, 1 \mathrm{H}), 1.79$ (s, $1 \mathrm{H}), 1.49$ (s, 3H), 1.34 (d, $J=7.2 \mathrm{~Hz}, 3 \mathrm{H}) .{ }^{13} \mathbf{C}$ NMR (150 MHz, $\left.\mathrm{CDCl}_{3}\right) \delta 152.0,144.3,136.2,127.8,126.6,126.3,123.6,114.9,107.3,105.9,74.6,47.2,36.3,26.5$, 16.6. HRMS (ESI-TOF) $(\mathrm{m} / \mathrm{z})$ : Calcd for $\mathrm{C}_{22} \mathrm{H}_{28} \mathrm{ONa}\left([\mathrm{M}+\mathrm{Na}]^{+}\right): 278.1521$; found: 278.1526 . Specific rotation $[\alpha]^{15} \mathrm{D}=-27.1,\left(\mathrm{c}=1.00, \mathrm{CHCl}_{3}\right) . \mathrm{HPLC}$ analysis $(\mathrm{IA}$, Hexane/IPA $=98 / 2,0.5 \mathrm{~mL} / \mathrm{min}, 250 \mathrm{~nm})$ indicated $94 \%$ ee: $\mathrm{tR}$ (major) $=15.18 \mathrm{~min}, \mathrm{tR}($ minor $)=20.19 \mathrm{~min}$.
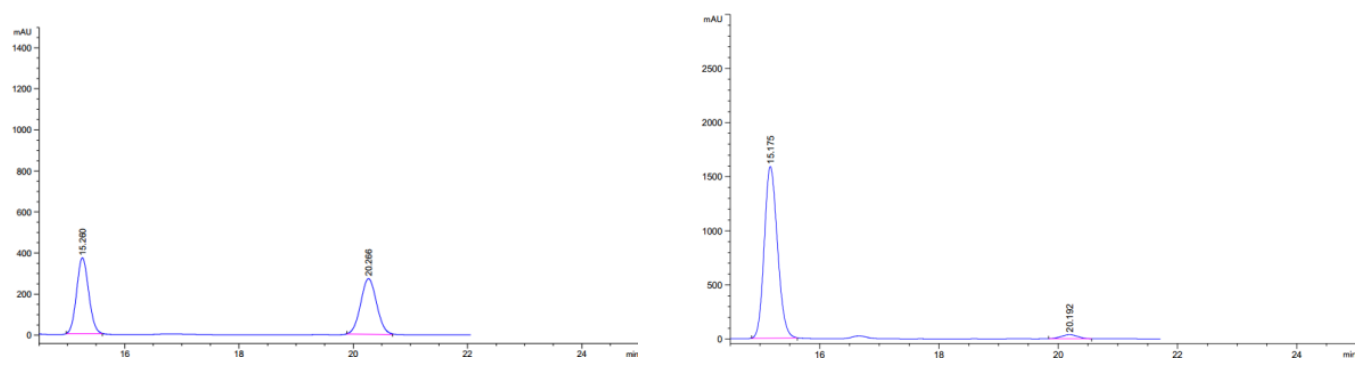

\begin{tabular}{llllc}
\hline $\begin{array}{l}\text { Peak } \\
\#\end{array}$ & $\begin{array}{l}\text { PetTime } \\
{[\mathrm{min}]}\end{array}$ & $\begin{array}{c}\text { Width } \\
{[\mathrm{min}]}\end{array}$ & $\begin{array}{l}\text { Area } \\
{[\mathrm{mAU} * \mathrm{~S}]}\end{array}$ & $\begin{array}{c}\text { Area } \\
\%\end{array}$ \\
\hline 1 & 15.26 & 0.2438 & 5413.40332 & 50.7746 \\
2 & 20.19 & 0.3234 & 5248.23525 & 49.2254 \\
\hline
\end{tabular}

\begin{tabular}{lllll}
\hline $\begin{array}{l}\text { Peak } \\
\#\end{array}$ & $\begin{array}{l}\text { PetTime } \\
{[\mathrm{min}]}\end{array}$ & $\begin{array}{l}\text { Width } \\
{[\mathrm{min}]}\end{array}$ & $\begin{array}{l}\text { Area } \\
{[\mathrm{mAU} * \mathrm{~S}]}\end{array}$ & $\begin{array}{c}\text { Area } \\
\%\end{array}$ \\
\hline 1 & 15.18 & 0.2562 & $2.44066 \mathrm{e} 4$ & 97.1664 \\
2 & 20.19 & 0.3248 & 711.76404 & 2.8336 \\
\hline
\end{tabular}

\section{(R)-1-((R)-3-phenylbut-3-en-2-yl)-2,3-dihydro-1H-inden-1-ol}

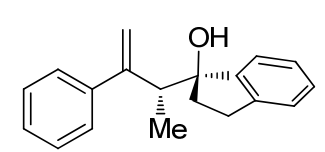

$3 t$

Prepared according to general procedure. In a nitrogen-filled glovebox, a flame-dried screw-cap reaction tube equipped with a Teflon-coated magnetic stir bar were charged with $\mathrm{Cu}(\mathrm{OAc})_{2}(1.8 \mathrm{mg}, 10.0 \mu \mathrm{mol}),(R, R)-\mathrm{Ph}-\mathrm{BPE}(6.1$ $\mathrm{mg}, 12.0 \mu \mathrm{mol})$, and anhydrous THF $(1 \mathrm{~mL})$, dimethoxymethylsilane (DMMS, 3 equiv) were subsequently added. The resulting mixture was stirred for $2 \mathrm{~min}$ and then buta-1,3-dien-2-ylbenzene ( $52.0 \mathrm{mg}, 0.4 \mathrm{mmol}$ ) and 2,3-dihydro-1H-inden-1-one (26.4 mg, 0.2 
mmol) were added, respectively. The vial was removed from the glove box, and the mixture was stirred at $20{ }^{\circ} \mathrm{C}$ for $18 \mathrm{~h}$. After $18 \mathrm{~h}$ the cap was removed. The solution was quenched by adding saturated ammonium fluoride in methanol using a $5 \mathrm{~mL}$ syringe $(3 \mathrm{~mL})$ and stirred for $30 \mathrm{~min}$. After removal of solvent under reduced pressure with aid of a rotary evaporator, the diastereomeric ratio (dr) was assessed by ${ }^{1} \mathrm{H}$ NMR in $\mathrm{CDCl}_{3}$, The resulting crude product was purified by flash column chromatography on silica gel (diethyl ether /petroleum ether, 1:10) to obtain product in $75 \%(39.6 \mathrm{mg})$ yield as a colorless oil. ${ }^{1} \mathbf{H}$ NMR $\left(600 \mathrm{MHz}, \mathrm{CDCl}_{3}\right) \delta 7.24-7.19(\mathrm{~m}, 6 \mathrm{H}), 7.14-7.12(\mathrm{~m}, 2 \mathrm{H}), 7.05-7.02(\mathrm{~m}, 1 \mathrm{H}), 5.32$ (s, 1H), $5.28(\mathrm{~s}, 1 \mathrm{H}), 3.20(\mathrm{q}, J=7.2 \mathrm{~Hz}, 1 \mathrm{H}), 2.82-2.77(\mathrm{~m}, 1 \mathrm{H}), 2.71-2.65(\mathrm{~m}, 1 \mathrm{H}), 2.45-2.41(\mathrm{~m}$, 1H), $2.02(\mathrm{~s}, 1 \mathrm{H}), 1.93-1.88(\mathrm{~m}, 1 \mathrm{H}), 1.36(\mathrm{~d}, J=7.2 \mathrm{~Hz}, 3 \mathrm{H}) .{ }^{13} \mathbf{C}$ NMR $\left(150 \mathrm{MHz}, \mathrm{CDCl}_{3}\right) \delta 151.8$, 146.6, 144.6, 142.7, 128.1, 127.9, 127.0, 126.3, 125.9, 124.69, 124.49, 115.4, 85.7, 45.3, 39.1, 29.9, 15.9. HRMS (ESI-TOF) $(\mathrm{m} / \mathrm{z})$ : Calcd for $\mathrm{C}_{19} \mathrm{H}_{20} \mathrm{ONa}\left([\mathrm{M}+\mathrm{Na}]^{+}\right)$: 287.1407; found: 287.1412. Specific rotation $[\alpha]^{20}{ }_{\mathrm{D}}=-37.9,\left(\mathrm{c}=1.00, \mathrm{CHCl}_{3}\right)$. HPLC analysis $(\mathrm{IC}, \mathrm{Hexane} / \mathrm{IPA}=97 / 3,0.8 \mathrm{~mL} / \mathrm{min}, 250 \mathrm{~nm})$ indicated $94 \%$ ee: $\mathrm{tR}$ (major) $=8.28 \mathrm{~min}, \mathrm{tR}($ minor $)=9.59 \mathrm{~min}$.
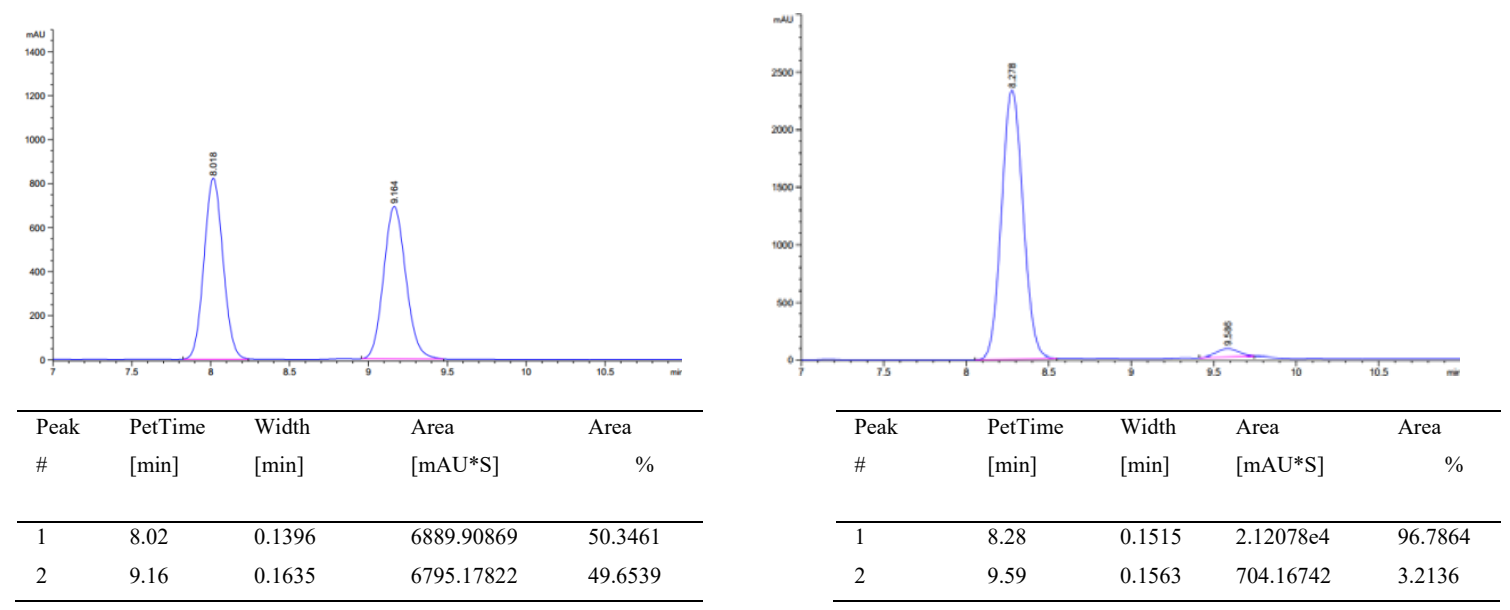

\section{(R)-2-(3-phenylbut-3-en-2-yl)-2,3-dihydro-1H-inden-2-ol}

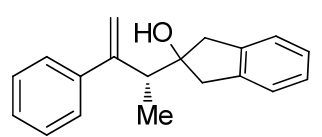

3u

Prepared according to general procedure. In a nitrogen-filled glovebox, a flame-dried screw-cap reaction tube equipped with a Teflon-coated magnetic stir bar were charged with $\mathrm{Cu}(\mathrm{OAc})_{2}(1.8 \mathrm{mg}, 10.0 \mu \mathrm{mol}),(R, R)-\mathrm{Ph}-\mathrm{BPE}(6.1$ $\mathrm{mg}, 12.0 \mu \mathrm{mol})$, and anhydrous THF ( $1 \mathrm{~mL}$ ), dimethoxymethylsilane (DMMS, 3 equiv) were subsequently added. The resulting mixture was stirred for $2 \mathrm{~min}$ and then buta-1,3-dien-2ylbenzene (52.0 mg, $0.4 \mathrm{mmol})$ and $1 \mathrm{H}$-inden-2(3H)-one (26.4 mg, $0.2 \mathrm{mmol})$ were added, respectively. The vial was removed from the glove box, and the mixture was stirred at $20{ }^{\circ} \mathrm{C}$ for $18 \mathrm{~h}$. After $18 \mathrm{~h}$ the cap was removed. The solution was quenched by adding saturated ammonium fluoride in methanol using a $5 \mathrm{~mL}$ syringe $(3 \mathrm{~mL})$ and stirred for $30 \mathrm{~min}$. After removal of solvent under reduced pressure with aid of a rotary evaporator, the diastereomeric ratio (dr) was assessed by ${ }^{1} \mathrm{H} \mathrm{NMR}$ in $\mathrm{CDCl}_{3}$, The resulting crude product was purified by flash column chromatography on silica gel (diethyl ether/petroleum ether, 1:10) to obtain product in $76 \%(40.1 \mathrm{mg})$ yield as a colorless oil. ${ }^{1} \mathbf{H}$ NMR $\left(600 \mathrm{MHz}, \mathrm{CDCl}_{3}\right) \delta 7.41(\mathrm{~d}$, $J=7.6 \mathrm{~Hz}, 2 \mathrm{H}), 7.35(\mathrm{t}, J=7.6 \mathrm{~Hz}, 2 \mathrm{H}), 7.29$ (t, $J=7.6 \mathrm{~Hz}, 1 \mathrm{H}), 7.19(\mathrm{~d}, J=7.2 \mathrm{~Hz}, 1 \mathrm{H}), 7.13-7.08$ $(\mathrm{m}, 2 \mathrm{H}), 7.05(\mathrm{~d}, J=7.2 \mathrm{~Hz}, 1 \mathrm{H}), 5.43(\mathrm{~s}, 1 \mathrm{H}), 5.33(\mathrm{~s}, 1 \mathrm{H}), 3.03(\mathrm{q}, J=7.2 \mathrm{~Hz}, 1 \mathrm{H}), 3.01(\mathrm{~m}, 2 \mathrm{H}), 2.77$ (d, $J=16.2 \mathrm{~Hz}, 1 \mathrm{H}), 2.53(\mathrm{~d}, J=16.2 \mathrm{~Hz}, 1 \mathrm{H}), 1.79(\mathrm{~s}, 1 \mathrm{H}), 1.40(\mathrm{~d}, J=7.2 \mathrm{~Hz}, 3 \mathrm{H}) .{ }^{13} \mathbf{C}$ NMR $(150$ 
$\left.\mathrm{MHz}, \mathrm{CDCl}_{3}\right) \delta 152.1,144.2,141.5,141.2,128.5,127.4,126.6,126.39,126.36,124.83,124.80,115.2$, 83.7, 47.2, 46.7, 46.0, 16.4. HRMS (ESI-TOF) $(\mathrm{m} / \mathrm{z})$ : Calcd for $\mathrm{C}_{19} \mathrm{H}_{20} \mathrm{ONa}\left([\mathrm{M}+\mathrm{Na}]^{+}\right)$: 287.1412; found: 287.1416 . Specific rotation $[\alpha]^{15} \mathrm{D}=-29.3,\left(\mathrm{c}=1.00, \mathrm{CHCl}_{3}\right)$. HPLC analysis (IA, Hexane/IPA = $98 / 2,0.5 \mathrm{~mL} / \mathrm{min}, 250 \mathrm{~nm}$ ) indicated $94 \%$ ee: $\mathrm{tR}$ (major) $=30.12 \mathrm{~min}$, $\mathrm{tR}$ (minor) $=40.55 \mathrm{~min}$.
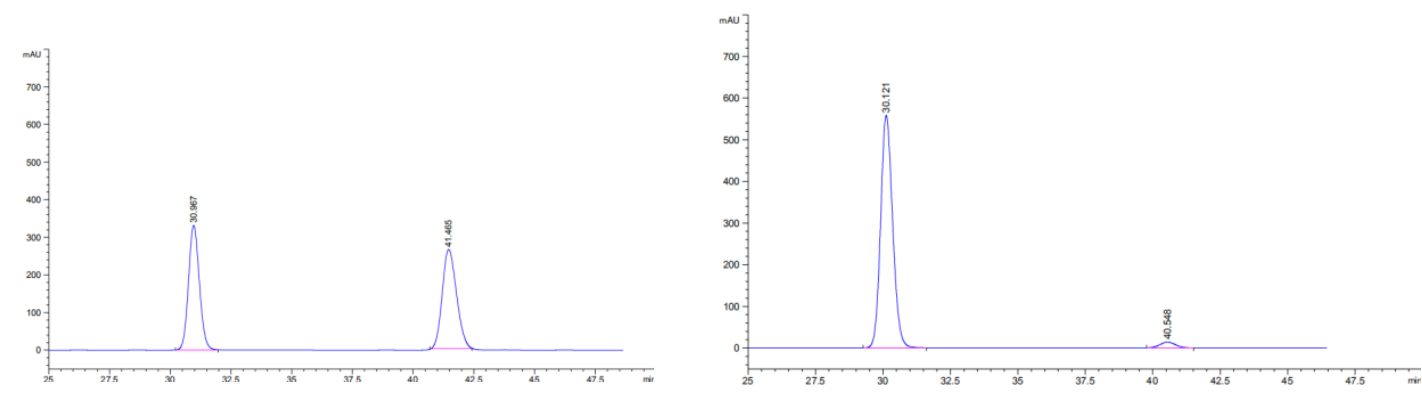

\begin{tabular}{lllll}
\hline $\begin{array}{l}\text { Peak } \\
\#\end{array}$ & $\begin{array}{l}\text { PetTime } \\
{[\mathrm{min}]}\end{array}$ & $\begin{array}{c}\text { Width } \\
{[\mathrm{min}]}\end{array}$ & $\begin{array}{l}\text { Area } \\
{\left[\mathrm{mAU}^{*} \mathrm{~S}\right]}\end{array}$ & $\begin{array}{c}\text { Area } \\
\%\end{array}$ \\
\hline 1 & 30.97 & 0.5146 & $1.03385 \mathrm{e} 4$ & 48.9100 \\
2 & 41.47 & 0.6812 & $1.07993 \mathrm{e} 4$ & 51.0900 \\
\hline
\end{tabular}

\begin{tabular}{lllll}
\hline $\begin{array}{l}\text { Peak } \\
\#\end{array}$ & $\begin{array}{l}\text { PetTime } \\
{[\mathrm{min}]}\end{array}$ & $\begin{array}{l}\text { Width } \\
{[\mathrm{min}]}\end{array}$ & $\begin{array}{l}\text { Area } \\
{[\mathrm{mAU} * \mathrm{~S}]}\end{array}$ & $\begin{array}{c}\text { Area } \\
\%\end{array}$ \\
\hline 1 & 30.12 & 0.4983 & $1.73090 \mathrm{e} 4$ & 96.8588 \\
2 & 40.55 & 0.6740 & 561.34869 & 3.1412 \\
\hline
\end{tabular}

(2R,3R)-2-cyclohexyl-3-methyl-4-phenylpent-4-en-2-ol

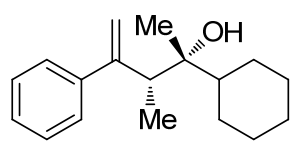

$3 \mathbf{v}$

Prepared according to general procedure. In a nitrogen-filled glovebox, a flamedried screw-cap reaction tube equipped with a Teflon-coated magnetic stir bar were charged with $\mathrm{Cu}(\mathrm{OAc})_{2}(1.8 \mathrm{mg}, 10.0 \mu \mathrm{mol}),(R, R)-\mathrm{Ph}-\mathrm{BPE}(6.1 \mathrm{mg}, 12.0$ $\mu \mathrm{mol})$, and anhydrous THF ( $1 \mathrm{~mL}$ ), dimethoxymethylsilane (DMMS, 3 equiv) were subsequently added. The resulting mixture was stirred for $2 \mathrm{~min}$ and then buta-1,3-dien-2-ylbenzene (52.0 $\mathrm{mg}, 0.4 \mathrm{mmol})$ and 1-cyclohexylethanone $(25.2 \mathrm{mg}, 0.2 \mathrm{mmol})$ were added, respectively. The vial was removed from the glove box, and the mixture was stirred at $20{ }^{\circ} \mathrm{C}$ for $18 \mathrm{~h}$. After $18 \mathrm{~h}$ the cap was removed. The solution was quenched by adding saturated ammonium fluoride in methanol using a $5 \mathrm{~mL}$ syringe $(3 \mathrm{~mL})$ and stirred for $30 \mathrm{~min}$. After removal of solvent under reduced pressure with aid of a rotary evaporator, the diastereomeric ratio (dr) was assessed by ${ }^{1} \mathrm{H}$ NMR in $\mathrm{CDCl}_{3}$, The resulting crude product was purified by flash column chromatography on silica gel (diethyl ether /petroleum ether, 1:10) to obtain product in $45 \%(23.2 \mathrm{mg})$ yield as a colorless oil. ${ }^{1} \mathbf{H}$ NMR (600 $\left.\mathrm{MHz}, \mathrm{CDCl}_{3}\right) \delta 7.37-7.34(\mathrm{~m}, 2 \mathrm{H}), 7.31(\mathrm{t}, J=7.6 \mathrm{~Hz}, 2 \mathrm{H}), 7.26-7.25(\mathrm{~m}, 1 \mathrm{H}), 5.40(\mathrm{~s}, 1 \mathrm{H}), 5.24(\mathrm{~s}$, $1 \mathrm{H}), 3.03(\mathrm{q}, J=7.2 \mathrm{~Hz}, 1 \mathrm{H}), 1.86(\mathrm{~m}, 1 \mathrm{H}), 1.78-1.73(\mathrm{~m}, 2 \mathrm{H}), 1.68-1.64(\mathrm{~m}, 2 \mathrm{H}), 1.57-1.52(\mathrm{~m}$, 2H), $1.24(\mathrm{~d}, J=7.2 \mathrm{~Hz}, 4 \mathrm{H}), 1.18-1.08(\mathrm{~m}, 2 \mathrm{H}), 1.03-0.93(\mathrm{~m}, 1 \mathrm{H}), 0.94-0.87(\mathrm{~m}, 1 \mathrm{H}), 0.84(\mathrm{~s}$, $3 \mathrm{H}) .{ }^{13} \mathbf{C}$ NMR $\left(150 \mathrm{MHz}, \mathrm{CDCl}_{3}\right) \delta 153.4,145.1,128.4,127.1,126.3,115.4,75.5,44.3,43.4,28.0,27.0$, 26.9, 26.7, 22.2, 15.4. HRMS (ESI-TOF) $(\mathrm{m} / \mathrm{z})$ : Calcd for $\mathrm{C}_{18} \mathrm{H}_{26} \mathrm{ONa}\left([\mathrm{M}+\mathrm{Na}]^{+}\right): 281.1881$; found: 281.1883. Specific rotation $[\alpha]^{14} \mathrm{D}=-22.5,\left(\mathrm{c}=1.00, \mathrm{CHCl}_{3}\right)$. HPLC analysis (IA, Hexane/IPA $=98 / 2$, $0.5 \mathrm{~mL} / \mathrm{min}, 250 \mathrm{~nm}$ ) indicated $78 \%$ ee: $\mathrm{tR}$ (major) $=12.68 \mathrm{~min}$, $\mathrm{tR}($ minor $)=14.25 \mathrm{~min}$. 

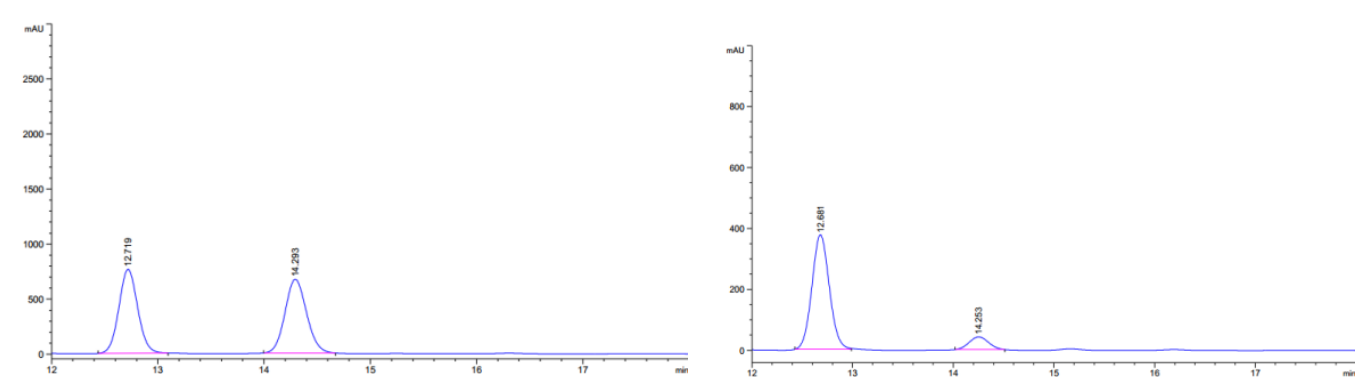

\begin{tabular}{llllc}
\hline $\begin{array}{l}\text { Peak } \\
\#\end{array}$ & $\begin{array}{l}\text { PetTime } \\
{[\mathrm{min}]}\end{array}$ & $\begin{array}{l}\text { Width } \\
{[\mathrm{min}]}\end{array}$ & $\begin{array}{l}\text { Area } \\
{[\mathrm{mAU} * \mathrm{~S}]}\end{array}$ & $\begin{array}{c}\text { Area } \\
\%\end{array}$ \\
\hline 1 & 12.72 & 0.2053 & 9379.31348 & 49.8485 \\
2 & 14.29 & 0.2351 & 9436.30762 & 50.1515 \\
\hline
\end{tabular}

\begin{tabular}{lllll}
\hline $\begin{array}{l}\text { Peak } \\
\#\end{array}$ & $\begin{array}{l}\text { PetTime } \\
{[\mathrm{min}]}\end{array}$ & $\begin{array}{l}\text { Width } \\
{[\mathrm{min}]}\end{array}$ & $\begin{array}{l}\text { Area } \\
{[\mathrm{mAU} * \mathrm{~S}]}\end{array}$ & \multicolumn{1}{c}{$\begin{array}{c}\text { Area } \\
\%\end{array}$} \\
\hline 1 & 12.68 & 0.2004 & 4519.53418 & 89.0105 \\
2 & 14.25 & 0.2227 & 557.99622 & 10.9895 \\
\hline
\end{tabular}

\section{(3S,4R)-4-methyl-3,5-diphenylhex-5-en-3-ol}

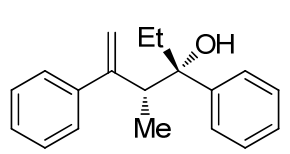

$3 \mathbf{w}$

Prepared according to general procedure. In a nitrogen-filled glovebox, a flamedried screw-cap reaction tube equipped with a Teflon-coated magnetic stir bar were charged with $\mathrm{Cu}(\mathrm{OAc})_{2}(1.8 \mathrm{mg}, 10.0 \mu \mathrm{mol}),(R, R)-\mathrm{Ph}-\mathrm{BPE}(6.1 \mathrm{mg}, 12.0$ $\mu \mathrm{mol})$, and anhydrous THF ( $1 \mathrm{~mL}$ ), dimethoxymethylsilane (DMMS, 3 equiv) were subsequently added. The resulting mixture was stirred for $2 \mathrm{~min}$ and then buta-1,3-dien-2-ylbenzene $(52.0 \mathrm{mg}, 0.4 \mathrm{mmol})$ and propiophenone $(26.8 \mathrm{mg}, 0.2 \mathrm{mmol})$ were added, respectively. The vial was removed from the glove box, and the mixture was stirred at $20{ }^{\circ} \mathrm{C}$ for $18 \mathrm{~h}$. After $18 \mathrm{~h}$ the cap was removed. The solution was quenched by adding saturated ammonium fluoride in methanol using a $5 \mathrm{~mL}$ syringe $(3 \mathrm{~mL})$ and stirred for $30 \mathrm{~min}$. After removal of solvent under reduced pressure with aid of a rotary evaporator, the diastereomeric ratio (dr) was assessed by ${ }^{1} \mathrm{H} \mathrm{NMR} \mathrm{in} \mathrm{CDCl}_{3}$, The resulting crude product was purified by flash column chromatography on silica gel (diethyl ether /petroleum ether, 1:10) to obtain product (as a mixture of diastereomers, dr: $2.5: 1$ ) in $92 \%$ (53.2 $\mathrm{mg}$ ) yield as a colorless oil and the major diastereomer was obtained in $43 \%(22.8 \mathrm{mg})$ yield as a colorless oil. Maior doastereomer: ${ }^{1} \mathbf{H}$ NMR $\left(600 \mathrm{MHz}, \mathrm{CDCl}_{3}\right) \delta 7.45(\mathrm{~d}, J=7.2 \mathrm{~Hz}, 2 \mathrm{H}), 7.40-7.27(\mathrm{~m}, 7 \mathrm{H})$, $7.24-7.20(\mathrm{~m}, 1 \mathrm{H}), 5.45(\mathrm{~s}, 1 \mathrm{H}), 5.25(\mathrm{~s}, 1 \mathrm{H}), 3.27(\mathrm{q}, J=7.2 \mathrm{~Hz}, 1 \mathrm{H}), 1.83(\mathrm{~s}, 1 \mathrm{H}), 1.61-1.56(\mathrm{~m}$, 2H), $0.98(\mathrm{~d}, J=7.2 \mathrm{~Hz}, 3 \mathrm{H}), 0.48(\mathrm{t}, J=7.2 \mathrm{~Hz}, 3 \mathrm{H}) .{ }^{13} \mathbf{C}$ NMR $\left(151 \mathrm{MHz}, \mathrm{CDCl}_{3}\right) \delta 152.2,145.0$, 144.3, 129.0, 127.8, 127.3, 126.4, 126.1, 125.6, 115.4, 79.2, 48.1, 34.9, 16.6, 7.9. HRMS (ESI-TOF) $(\mathrm{m} / \mathrm{z})$ : Calcd for $\mathrm{C}_{19} \mathrm{H}_{22} \mathrm{ONa}\left([\mathrm{M}+\mathrm{Na}]^{+}\right): 289.1568$; found: 289.1572 . Specific rotation $[\alpha]^{20}{ }_{\mathrm{D}}=-7.8$, $(\mathrm{c}$ $=1.00, \mathrm{CHCl}_{3}$ ). HPLC analysis (IB, Hexane/IPA $=98.5 / 1.5,0.4 \mathrm{~mL} / \mathrm{min}, 250 \mathrm{~nm}$ ) indicated $21 \%$ ee: tR $($ major $)=8.28 \mathrm{~min}, \mathrm{tR}($ minor $)=9.59 \mathrm{~min}$.
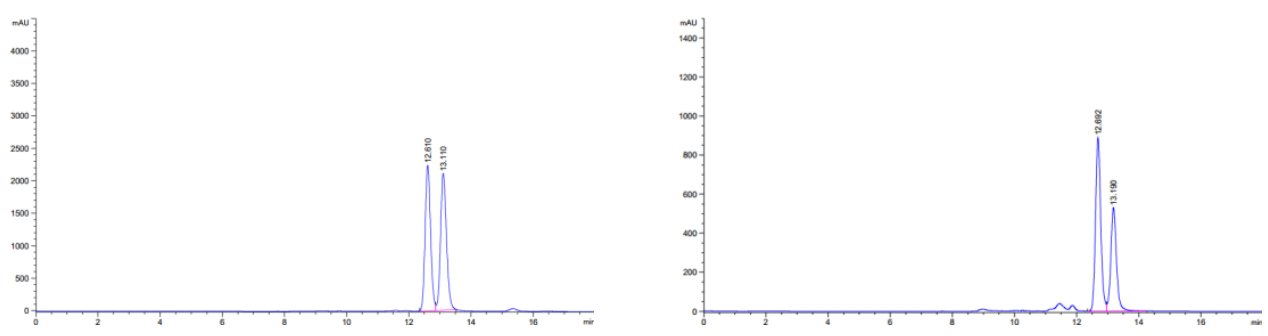


\begin{tabular}{lllll}
\hline $\begin{array}{l}\text { Peak } \\
\#\end{array}$ & $\begin{array}{l}\text { PetTime } \\
{[\mathrm{min}]}\end{array}$ & $\begin{array}{c}\text { Width } \\
{[\mathrm{min}]}\end{array}$ & $\begin{array}{l}\text { Area } \\
{[\mathrm{mAU} * \mathrm{~S}]}\end{array}$ & $\begin{array}{c}\text { Area } \\
\%\end{array}$ \\
\hline 1 & 12.61 & 0.2043 & $2.74546 \mathrm{e} 4$ & 49.4976 \\
2 & 13.11 & 0.2216 & $2.80119 \mathrm{e} 4$ & 50.5024 \\
\hline
\end{tabular}

\begin{tabular}{lllll}
\hline $\begin{array}{l}\text { Peak } \\
\#\end{array}$ & $\begin{array}{l}\text { PetTime } \\
{[\mathrm{min}]}\end{array}$ & $\begin{array}{l}\text { Width } \\
{[\mathrm{min}]}\end{array}$ & $\begin{array}{l}\text { Area } \\
{[\mathrm{mAU} * \mathrm{~S}]}\end{array}$ & $\begin{array}{c}\text { Area } \\
\%\end{array}$ \\
\hline 1 & 12.69 & 0.1795 & $1.04029 \mathrm{e} 4$ & 60,6285 \\
2 & 13.19 & 0.1925 & 6755.50586 & 39.3715 \\
\hline
\end{tabular}

\section{(2S,3R)-4-(4-methoxyphenyl)-3-methyl-2-phenylpent-4-en-2-ol}

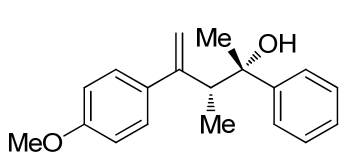

$3 y$

Prepared according to general procedure. In a nitrogen-filled glovebox, a flame-dried screw-cap reaction tube equipped with a Teflon-coated magnetic stir bar were charged with $\mathrm{Cu}(\mathrm{OAc})_{2}(1.8 \mathrm{mg}, 10.0 \mu \mathrm{mol}),(R, R)-\mathrm{Ph}-\mathrm{BPE}(6,1$ $\mathrm{mg}, 12.0 \mu \mathrm{mol})$, and anhydrous THF $(1 \mathrm{~mL})$, dimethoxymethylsilane (DMMS, 3 equiv) were subsequently added. The resulting mixture was stirred for $2 \mathrm{~min}$ and then 1-(buta-1,3-dien-2-yl)-4-methoxybenzene $(64.0 \mathrm{mg}, 0.4 \mathrm{mmol})$ and acetophenone ( $24.0 \mathrm{mg}, 0.2 \mathrm{mmol}$ ) were added, respectively. The vial was removed from the glove box, and the mixture was stirred at $20{ }^{\circ} \mathrm{C}$ for $18 \mathrm{~h}$. After $18 \mathrm{~h}$ the cap was removed. The solution was quenched by adding saturated ammonium fluoride in methanol using a $5 \mathrm{~mL}$ syringe $(3 \mathrm{~mL})$ and stirred for $30 \mathrm{~min}$. After removal of solvent under reduced pressure with aid of a rotary evaporator, the diastereomeric ratio (dr) was assessed by ${ }^{1} \mathrm{H}$ NMR in $\mathrm{CDCl}_{3}$, The resulting crude product was purified by flash column chromatography on silica gel (diethyl ether /petroleum ether, 1:10) to obtain product in $93 \%(52.5 \mathrm{mg})$ yield as a colorless oil. ${ }^{1} \mathbf{H}$ NMR $\left(600 \mathrm{MHz}, \mathrm{CDCl}_{3}\right) \delta 7.45-7.43(\mathrm{~m}, 2 \mathrm{H}), 7.38-7.36(\mathrm{~m}, 2 \mathrm{H}), 7.33-$ $7.30(\mathrm{~m}, 2 \mathrm{H}), 7.23-7.20(\mathrm{~m}, 1 \mathrm{H}), 6.88(\mathrm{~d}, J=8.4 \mathrm{~Hz}, 2 \mathrm{H}), 5.39(\mathrm{~s}, 1 \mathrm{H}), 5.19(\mathrm{~s}, 1 \mathrm{H}), 3.82(\mathrm{~s}, 3 \mathrm{H}), 3.23$ (q, $J=7.2 \mathrm{~Hz}, 1 \mathrm{H}), 2.05(\mathrm{~s}, 1 \mathrm{H}), 1.31(\mathrm{~s}, 3 \mathrm{H}), 0.99(\mathrm{~d}, J=7.2 \mathrm{~Hz}, 3 \mathrm{H}) .{ }^{13} \mathbf{C} \mathbf{N M R}\left(151 \mathrm{MHz}, \mathrm{CDCl}_{3}\right) \delta$ 159.0, 151.3, 147.1, 137.2, 127.9, 127.5, 126.2, 124.9, 113.9, 113.8, 76.0, 55.3, 48.1, 30.8, 16.3. HRMS (ESI-TOF) $(\mathrm{m} / \mathrm{z})$ : Calcd for $\mathrm{C}_{19} \mathrm{H}_{22} \mathrm{O}_{2} \mathrm{Na}\left([\mathrm{M}+\mathrm{Na}]^{+}\right)$: 305.1512 ; found: 305.1516 . Specific rotation $[\alpha]^{15} \mathrm{D}=-31.2,\left(\mathrm{c}=1.00, \mathrm{CHCl}_{3}\right)$. HPLC analysis (IA, Hexane/IPA $\left.=98 / 2,0.5 \mathrm{~mL} / \mathrm{min}, 250 \mathrm{~nm}\right)$ indicated $90 \%$ ee: $\operatorname{tR}($ major $)=19.76 \mathrm{~min}, \mathrm{tR}($ minor $)=26.52 \mathrm{~min}$.
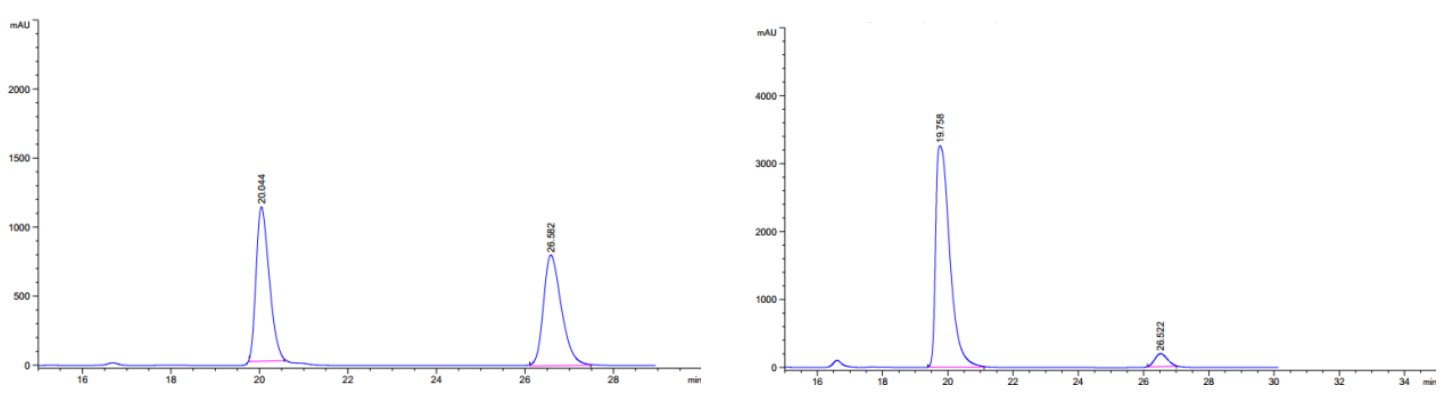

\begin{tabular}{llllc}
\hline $\begin{array}{l}\text { Peak } \\
\#\end{array}$ & $\begin{array}{l}\text { PetTime } \\
{[\mathrm{min}]}\end{array}$ & $\begin{array}{l}\text { Width } \\
{[\mathrm{min}]}\end{array}$ & $\begin{array}{l}\text { Area } \\
{[\mathrm{mAU} * \mathrm{~S}]}\end{array}$ & $\begin{array}{c}\text { Area } \\
\%\end{array}$ \\
\hline 1 & 20.04 & 0.3435 & $2.30574 \mathrm{e} 4$ & 50.4065 \\
2 & 26.58 & 0.4712 & $2.26855 \mathrm{e} 4$ & 49.5935 \\
\hline
\end{tabular}

\begin{tabular}{llllr}
\hline $\begin{array}{l}\text { Peak } \\
\#\end{array}$ & $\begin{array}{l}\text { PetTime } \\
{[\mathrm{min}]}\end{array}$ & $\begin{array}{l}\text { Width } \\
{[\mathrm{min}]}\end{array}$ & \multicolumn{1}{l}{$\begin{array}{l}\text { Area } \\
{\left[\mathrm{mAU}^{*} \mathrm{~S}\right]}\end{array}$} & \multicolumn{1}{c}{ Area } \\
$\%$
\end{tabular}




\section{(2S,3R)-4-(3-methoxyphenyl)-3-methyl-2-phenylpent-4-en-2-ol}<smiles>C=C(c1cccc(OC)c1)[C@H](C)C(C)(O)c1ccccc1</smiles>

$3 z$

Prepared according to general procedure. In a nitrogen-filled glovebox, a flame-dried screw-cap reaction tube equipped with a Teflon-coated magnetic stir bar were charged with $\mathrm{Cu}(\mathrm{OAc})_{2}(1.8 \mathrm{mg}, 10.0 \mu \mathrm{mol}),(R, R)$ Ph-BPE (6.1 mg, $12.0 \mu \mathrm{mol})$, and anhydrous THF $(1 \mathrm{~mL})$, dimethoxymethylsilane (DMMS, 3 equiv) were subsequently added. The resulting mixture was stirred for $2 \mathrm{~min}$ and then 1-(buta-1,3-dien-2-yl)-3-methoxybenzene (64.0 mg, 0.4 $\mathrm{mmol}$ ) and acetophenone $(24.0 \mathrm{mg}, 0.2 \mathrm{mmol})$ were added, respectively. The vial was removed from the glove box, and the mixture was stirred at $20{ }^{\circ} \mathrm{C}$ for $18 \mathrm{~h}$. After $18 \mathrm{~h}$ the cap was removed. The solution was quenched by adding saturated ammonium fluoride in methanol using a $5 \mathrm{~mL}$ syringe $(3 \mathrm{~mL})$ and stirred for $30 \mathrm{~min}$. After removal of solvent under reduced pressure with aid of a rotary evaporator, the diastereomeric ratio (dr) was assessed by ${ }^{1} \mathrm{H} \mathrm{NMR}$ in $\mathrm{CDCl}_{3}$, The resulting crude product was purified by flash column chromatography on silica gel (diethyl ether /petroleum ether, 1:10) to obtain product in $85 \%$ (47.9 mg) yield as a colorless oil. ${ }^{1} \mathbf{H} \mathbf{~ N M R}\left(600 \mathrm{MHz}, \mathrm{CDCl}_{3}\right) \delta 7.43(\mathrm{~d}, J=7.8 \mathrm{~Hz}, 2 \mathrm{H}), 7.31$ (t, $J=7.2 \mathrm{~Hz}, 2 \mathrm{H}), 7.27(\mathrm{t}, J=7.8 \mathrm{~Hz}, 1 \mathrm{H}), 7.21(\mathrm{t}, J=7.2 \mathrm{~Hz}, 1 \mathrm{H}), 7.02(\mathrm{~d}, J=7.8 \mathrm{~Hz}, 1 \mathrm{H}), 6.96(\mathrm{~s}, 1 \mathrm{H})$, $6.84-6.82(\mathrm{~m}, 1 \mathrm{H}), 5.46(\mathrm{~s}, 1 \mathrm{H}), 5.27(\mathrm{~s}, 1 \mathrm{H}), 3.83(\mathrm{~s}, 3 \mathrm{H}), 3.24(\mathrm{q}, J=7.2 \mathrm{~Hz}, 1 \mathrm{H}), 2.00(\mathrm{~s}, 1 \mathrm{H}), 1.33$ $(\mathrm{s}, 3 \mathrm{H}), 1.00(\mathrm{~d}, J=7.2 \mathrm{~Hz}, 3 \mathrm{H}) .{ }^{13} \mathbf{C} \mathbf{N M R}\left(150 \mathrm{MHz}, \mathrm{CDCl}_{3}\right) \delta 159.6,151.8,147.1,146.4,129.4,127.9$, 126.3 124.9, 118.9, 115.5, 112.5, 112.3, 76.1, 55.2, 48.2, 30.7, 16.4. HRMS (ESI-TOF) (m/z): Calcd for $\mathrm{C}_{19} \mathrm{H}_{22} \mathrm{O}_{2} \mathrm{Na}\left([\mathrm{M}+\mathrm{Na}]^{+}\right)$: 305.1512; found: 305.1514. Specific rotation $[\alpha]^{15}{ }_{\mathrm{D}}=-29.9,\left(\mathrm{c}=1.00, \mathrm{CHCl}_{3}\right)$. HPLC analysis (IA, Hexane/IPA $=98 / 2,0.5 \mathrm{~mL} / \mathrm{min}, 250 \mathrm{~nm}$ ) indicated $95 \%$ ee: $\mathrm{tR}$ (major) $=21.95$ $\min , \mathrm{tR}($ minor $)=26.67 \mathrm{~min}$.
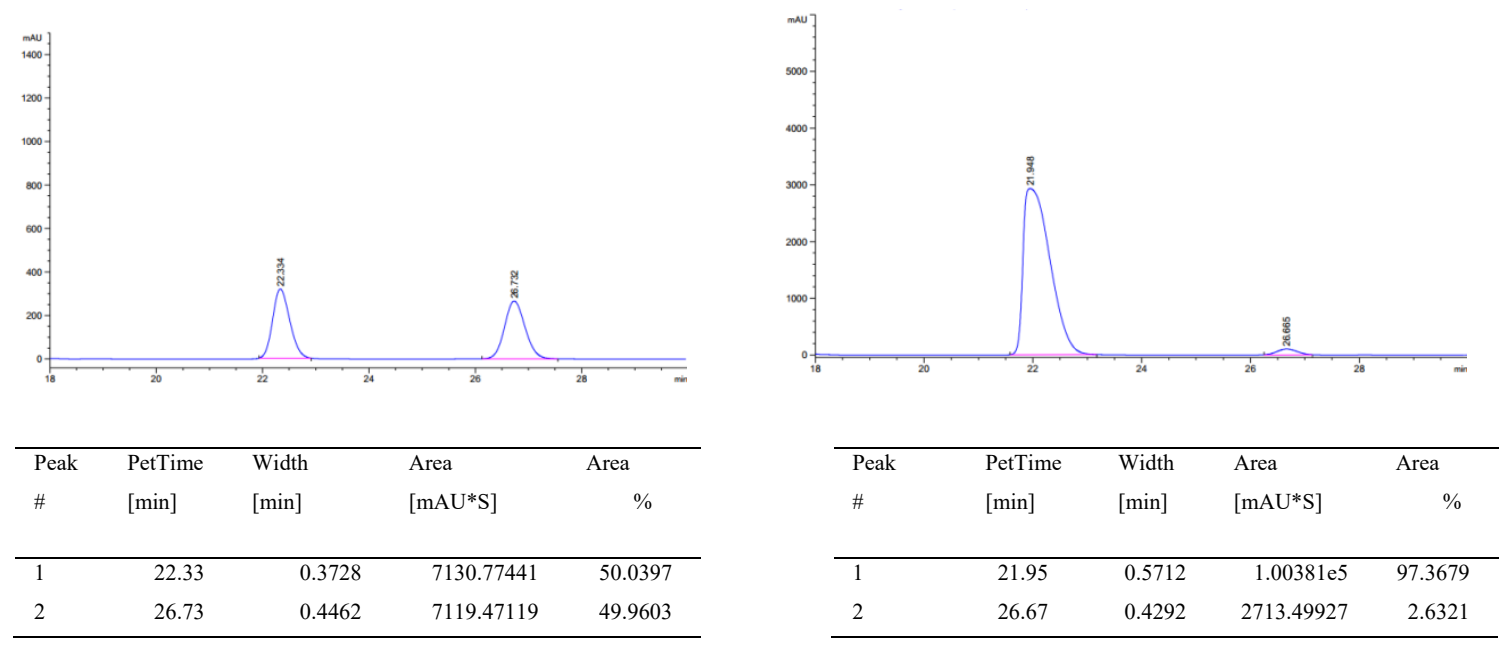

\section{(2S,3R)-4-(benzo[d] [1,3]dioxol-5-yl)-3-methyl-2-phenylpent-4-en-2-ol}<smiles>C=C(c1ccc2c(c1)OCO2)[C@H](C)[C@](C)(O)c1ccccc1</smiles>

3aa
Prepared according to general procedure. In a nitrogen-filled glovebox, a flame-dried screw-cap reaction tube equipped with a Teflon-coated magnetic stir bar were charged with $\mathrm{Cu}(\mathrm{OAc})_{2}(1.8 \mathrm{mg}, 10.0 \mu \mathrm{mol}),(R, R)-\mathrm{Ph}-\mathrm{BPE}(6.1$ $\mathrm{mg}, 12.0 \mu \mathrm{mol})$, and anhydrous THF (1 mL), dimethoxymethylsilane (DMMS, 3 equiv) were subsequently added. The resulting mixture was 
stirred for $2 \mathrm{~min}$ and then 5-(buta-1,3-dien-2-yl)benzo[d][1,3]dioxole $(69.6 \mathrm{mg}, 0.4 \mathrm{mmol})$ and acetophenone ( $24.0 \mathrm{mg}, 0.2 \mathrm{mmol}$ ) were added, respectively. The vial was removed from the glove box, and the mixture was stirred at $20{ }^{\circ} \mathrm{C}$ for $18 \mathrm{~h}$. After $18 \mathrm{~h}$ the cap was removed. The solution was quenched by adding saturated ammonium fluoride in methanol using a $5 \mathrm{~mL}$ syringe $(3 \mathrm{~mL})$ and stirred for $30 \mathrm{~min}$. After removal of solvent under reduced pressure with aid of a rotary evaporator, the diastereomeric ratio (dr) was assessed by ${ }^{1} \mathrm{H} N M R$ in $\mathrm{CDCl}_{3}$, The resulting crude product was purified by flash column chromatography on silica gel (diethyl ether /petroleum ether, 1:10) to obtain product in $92 \%(54.5 \mathrm{mg})$ yield as a colorless oil. ${ }^{1} \mathbf{H}$ NMR $\left(600 \mathrm{MHz}, \mathrm{CDCl}_{3}\right) \delta 7.43(\mathrm{~d}, J=7.8 \mathrm{~Hz}, 2 \mathrm{H}), 7.33-$ $7.30(\mathrm{~m}, 2 \mathrm{H}), 7.23-7.20(\mathrm{~m}, 1 \mathrm{H}), 6.91(\mathrm{~d}, J=7.2 \mathrm{~Hz}, 2 \mathrm{H}), 6.79(\mathrm{~d}, J=8.4 \mathrm{~Hz}, 1 \mathrm{H}), 5.97(\mathrm{~s}, 2 \mathrm{H}), 5.37$ (s, 1H), $5.18(\mathrm{~s}, 1 \mathrm{H}), 3.18(\mathrm{q}, J=7.2 \mathrm{~Hz}, 1 \mathrm{H}), 2.01(\mathrm{~s}, 1 \mathrm{H}), 1.33(\mathrm{~s}, 3 \mathrm{H}), 0.98(\mathrm{~d}, J=7.2 \mathrm{~Hz}, 3 \mathrm{H}) .{ }^{13} \mathbf{C}$ NMR $\left(150 \mathrm{MHz}, \mathrm{CDCl}_{3}\right) \delta 151.4,147.7,147.0,146.9,139.2,127.9,126.3,124.9,119.8,114.5,108.1$, 107.1, 101.1, 76.1, 48.4, 30.7, 16.4. HRMS (ESI-TOF) $(\mathrm{m} / \mathrm{z})$ : Calcd for $\mathrm{C}_{19} \mathrm{H}_{22} \mathrm{O}_{3} \mathrm{Na}\left([\mathrm{M}+\mathrm{Na}]^{+}\right)$: 321.1461; found: 321.1453 . Specific rotation $[\alpha]^{15} \mathrm{D}=-25.7,\left(\mathrm{c}=1.00, \mathrm{CHCl}_{3}\right)$. HPLC analysis (IA, Hexane/IPA $=98 / 2,0.5 \mathrm{~mL} / \mathrm{min}, 250 \mathrm{~nm}$ ) indicated $95 \%$ ee: $\mathrm{tR}$ (major) $=28.21 \mathrm{~min}, \mathrm{tR}($ minor $)=42.73$ $\min$.
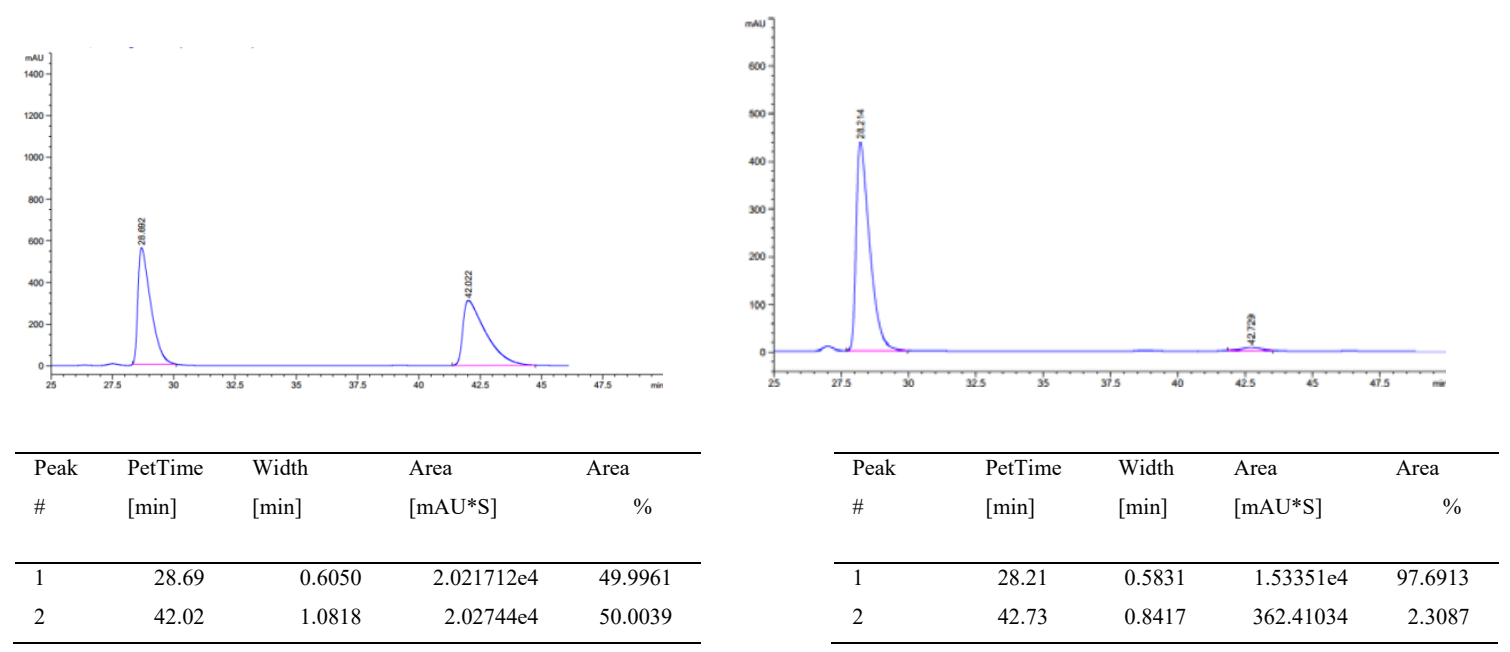

\section{(2S,3R)-4-([1,1'-biphenyl]-4-yl)-3-methyl-2-phenylpent-4-en-2-ol}

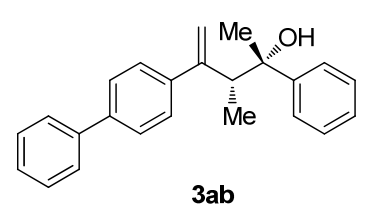

Prepared according to general procedure. In a nitrogen-filled glovebox, a flame-dried screw-cap reaction tube equipped with a Teflon-coated magnetic stir bar were charged with $\mathrm{Cu}(\mathrm{OAc})_{2}(1.8 \mathrm{mg}, 10.0 \mu \mathrm{mol}),(R, R)$ Ph-BPE (6.1 mg, $12.0 \mu \mathrm{mol})$, and anhydrous THF (1 $\mathrm{mL})$, dimethoxymethylsilane (DMMS, 3 equiv) were subsequently added. The resulting mixture was stirred for $2 \mathrm{~min}$ and then 4-(buta-1,3-dien-2-yl)-1,1'-biphenyl ( $82.4 \mathrm{mg}, 0.4 \mathrm{mmol}$ ) and acetophenone $(24.0 \mathrm{mg}, 0.2 \mathrm{mmol})$ were added, respectively. The vial was removed from the glove box, and the mixture was stirred at $20{ }^{\circ} \mathrm{C}$ for $18 \mathrm{~h}$. After $18 \mathrm{~h}$ the cap was removed. The solution was quenched by adding saturated ammonium fluoride in methanol using a $5 \mathrm{~mL}$ syringe $(3 \mathrm{~mL})$ and stirred for $30 \mathrm{~min}$. After removal of solvent under reduced pressure with aid of a rotary evaporator, the diastereomeric ratio (dr) was assessed by ${ }^{1} \mathrm{H} N M R$ in $\mathrm{CDCl}_{3}$, The resulting crude product was purified by flash column chromatography on silica gel (diethyl ether /petroleum ether, 1:10) to obtain product in 95\% (62.3 mg) yield as a white solid. m.p. $69-71{ }^{\circ} \mathrm{C} .{ }^{1} \mathbf{H} \mathbf{~ N M R}\left(600 \mathrm{MHz}, \mathrm{CDCl}_{3}\right) \delta 7.61(\mathrm{~d}, J=7.2$ $\mathrm{Hz}, 2 \mathrm{H}), 7.58$ (d, $J=8.2 \mathrm{~Hz}, 2 \mathrm{H}), 7.50(\mathrm{~d}, J=8.2 \mathrm{~Hz}, 2 \mathrm{H}), 7.46-7.43$ (m, 4H), $7.36-7.31$ (m, 3H), 
$7.23-7.20(\mathrm{~m}, 1 \mathrm{H}), 5.51(\mathrm{~s}, 1 \mathrm{H}), 5.30(\mathrm{~s}, 1 \mathrm{H}), 3.30(\mathrm{q}, J=7.2 \mathrm{~Hz}, 1 \mathrm{H}), 2.02(\mathrm{~s}, 1 \mathrm{H}), 1.36(\mathrm{~s}, 3 \mathrm{H}), 1.03$ $(\mathrm{d}, J=7.2 \mathrm{~Hz}, 3 \mathrm{H}) .{ }^{13} \mathbf{C} \mathbf{N M R}\left(150 \mathrm{MHz}, \mathrm{CDCl}_{3}\right) \delta 151.5,147.1,143.7,140.6,140.1,128.8,127.9,127.3$, 127.1, 127.0, 126.8, 126.3, 124.9, 115.3, 76.1, 48.1, 30.8, 16.4. HRMS (ESI-TOF) (m/z): Calcd for $\mathrm{C}_{24} \mathrm{H}_{24} \mathrm{ONa}\left([\mathrm{M}+\mathrm{Na}]^{+}\right): 351.1719$; found: 351.1720 . Specific rotation $[\alpha]^{15} \mathrm{D}=-27.1,\left(\mathrm{c}=1.00, \mathrm{CHCl}_{3}\right)$. HPLC analysis (IA, Hexane/IPA $=98 / 2,0.5 \mathrm{~mL} / \mathrm{min}, 250 \mathrm{~nm}$ ) indicated $92 \%$ ee: $\mathrm{tR}$ (major) $=23.60$ $\min , \mathrm{tR}($ minor $)=28.23 \mathrm{~min}$.

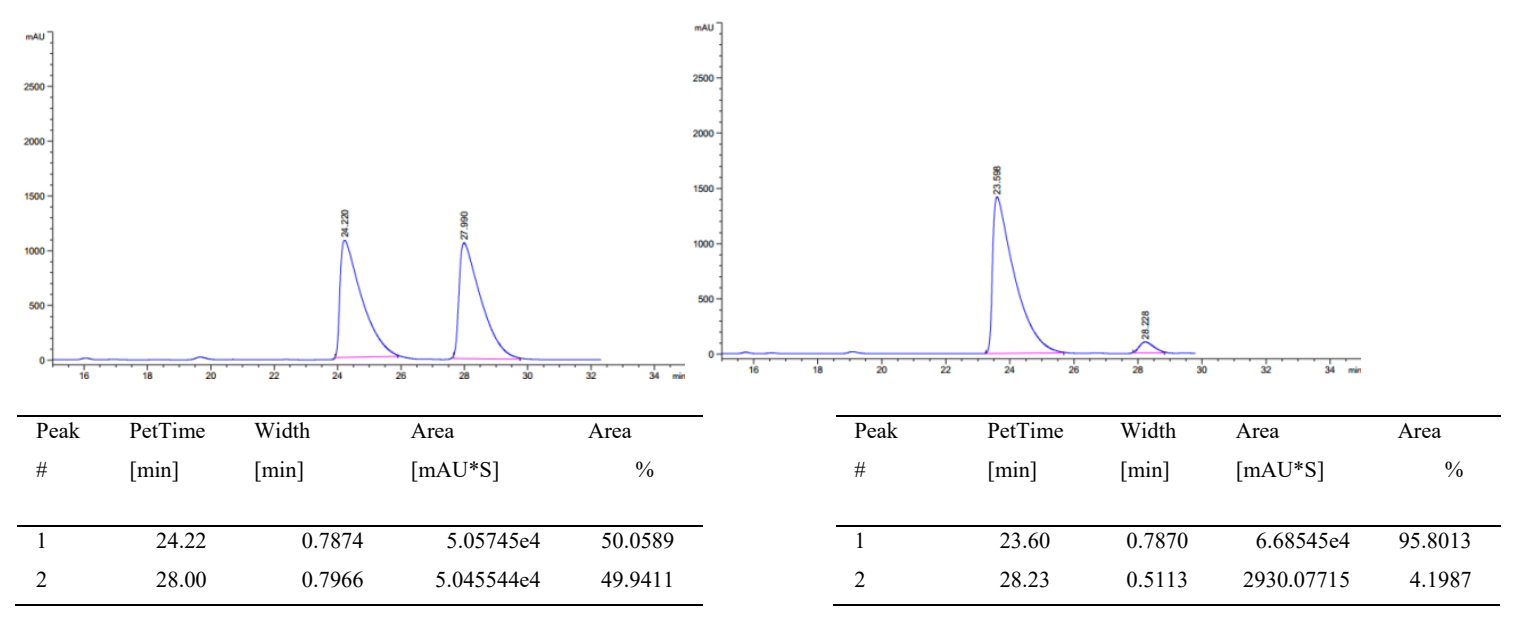

(2S,3R)-4-(4-isobutylphenyl)-3-methyl-2-phenylpent-4-en-2-ol

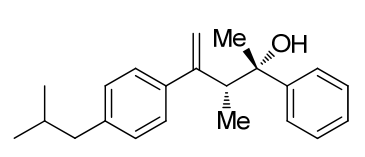

Prepared according to general procedure. In a nitrogen-filled glovebox, a flame-dried screw-cap reaction tube equipped with a Teflon-coated magnetic stir bar were charged with $\mathrm{Cu}(\mathrm{OAc})_{2}(1.8 \mathrm{mg}, 10.0 \mu \mathrm{mol}),(R, R)-$ Ph-BPE $(6.1 \mathrm{mg}, \quad 12.0 \mu \mathrm{mol})$, and anhydrous THF $(1 \mathrm{~mL})$, dimethoxymethylsilane (DMMS, 3 equiv) were subsequently added. The resulting mixture was stirred for $2 \mathrm{~min}$ and then 1-(buta-1,3-dien-2-yl)-4-isobutylbenzene (74.4 mg, 0.4 $\mathrm{mmol}$ ) and acetophenone $(24.0 \mathrm{mg}, 0.2 \mathrm{mmol})$ were added, respectively. The vial was removed from the glove box, and the mixture was stirred at $20{ }^{\circ} \mathrm{C}$ for $18 \mathrm{~h}$. After $18 \mathrm{~h}$ the cap was removed. The solution was quenched by adding saturated ammonium fluoride in methanol using a $5 \mathrm{~mL}$ syringe $(3 \mathrm{~mL})$ and stirred for $30 \mathrm{~min}$. After removal of solvent under reduced pressure with aid of a rotary evaporator, the diastereomeric ratio (dr) was assessed by ${ }^{1} \mathrm{H} N M R$ in $\mathrm{CDCl}_{3}$, The resulting crude product was purified by flash column chromatography on silica gel (diethyl ether /petroleum ether, 1:10) to obtain product in $84 \%(51.7 \mathrm{mg})$ yield as a colorless oil. ${ }^{1} \mathbf{H} \mathbf{~ N M R}\left(600 \mathrm{MHz}, \mathrm{CDCl}_{3}\right) \delta 7.44(\mathrm{~d}, J=7.2 \mathrm{~Hz}, 2 \mathrm{H}), 7.34-$ $7.30(\mathrm{~m}, 4 \mathrm{H}), 7.22-7.19(\mathrm{~m}, 1 \mathrm{H}), 7.12(\mathrm{~d}, J=7.8 \mathrm{~Hz}, 2 \mathrm{H}), 5.44(\mathrm{~s}, 1 \mathrm{H}), 5.23(\mathrm{~s}, 1 \mathrm{H}), 3.27(\mathrm{q}, J=7.2$ $\mathrm{Hz}, 1 \mathrm{H}), 2.47$ (d, J=7.2 Hz, 2H), 2.04 (s, 1H), $1.90-1.85$ (m, 1H), 1.31 (s, 3H), 1.00 (d, J=7.2 Hz, $3 \mathrm{H}), 0.91(\mathrm{~d}, J=6.6 \mathrm{~Hz}, 6 \mathrm{H}) .{ }^{13} \mathbf{C}$ NMR $\left(150 \mathrm{MHz}, \mathrm{CDCl}_{3}\right) \delta 151.8,147.1,142.1,140.9,129.2,127.9$, 126.2, 126.1, 124.9, 114.6, 76.1, 48.0, 45.0, 30.8, 30.2, 22.39, 22.37, 16.4. HRMS (ESI-TOF) (m/z): Calcd for $\mathrm{C}_{22} \mathrm{H}_{28} \mathrm{ONa}\left([\mathrm{M}+\mathrm{Na}]^{+}\right)$: 331.2032; found: 331.2042. Specific rotation $[\alpha]^{18}{ }_{\mathrm{D}}=-26.3,(\mathrm{c}=1.00$, $\mathrm{CHCl}_{3}$ ). HPLC analysis (IA, Hexane/IPA $=98 / 2,0.5 \mathrm{~mL} / \mathrm{min}, 250 \mathrm{~nm}$ ) indicated $93 \%$ ee: $\mathrm{tR}$ (major) $=$ $11.49 \mathrm{~min}, \mathrm{tR}($ minor $)=15.24 \mathrm{~min}$. 

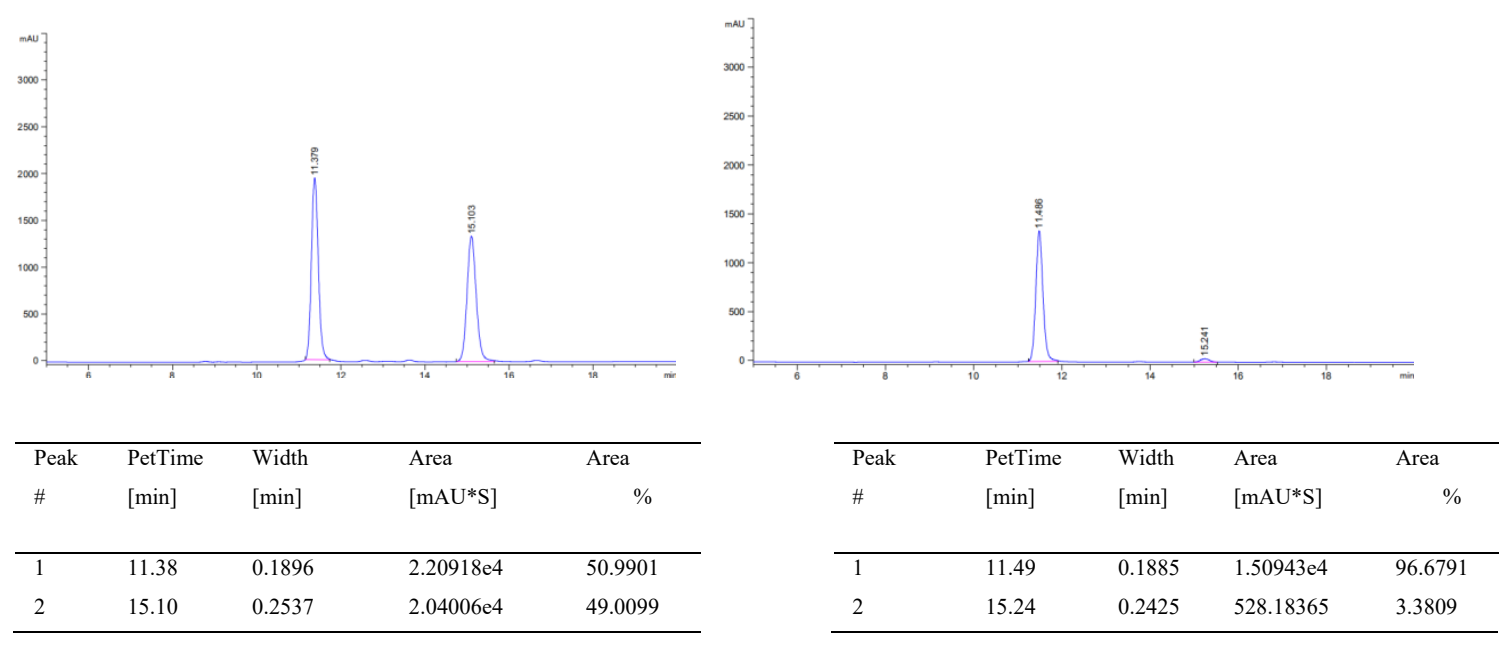

\section{(2S,3R)-3-methyl-4-(naphthalen-2-yl)-2-phenylpent-4-en-2-ol}

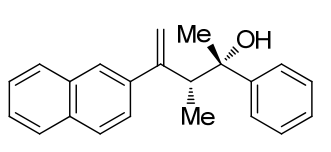

3ad

Prepared according to general procedure. In a nitrogen-filled glovebox, a flame-dried screw-cap reaction tube equipped with a Teflon-coated magnetic stir bar were charged with $\mathrm{Cu}(\mathrm{OAc})_{2}(1.8 \mathrm{mg}, 10.0 \mu \mathrm{mol}),(R, R)-\mathrm{Ph}-\mathrm{BPE}(6.1$ $\mathrm{mg}, 12.0 \mu \mathrm{mol})$, and anhydrous THF $(1 \mathrm{~mL})$, dimethoxymethylsilane (DMMS, 3 equiv) were subsequently added. The resulting mixture was stirred for $2 \mathrm{~min}$ and then 2-(buta-1,3-dien-2-yl) naphthalene (72.0 mg, $0.4 \mathrm{mmol}$ ) and acetophenone ( $24.0 \mathrm{mg}, 0.2 \mathrm{mmol}$ ) were added, respectively. The vial was removed from the glove box, and the mixture was stirred at $20{ }^{\circ} \mathrm{C}$ for $18 \mathrm{~h}$. After $18 \mathrm{~h}$ the cap was removed. The solution was quenched by adding saturated ammonium fluoride in methanol using a $5 \mathrm{~mL}$ syringe $(3 \mathrm{~mL})$ and stirred for $30 \mathrm{~min}$. After removal of solvent under reduced pressure with aid of a rotary evaporator, the diastereomeric ratio (dr) was assessed by ${ }^{1} \mathrm{H}$ NMR in $\mathrm{CDCl}_{3}$, The resulting crude product was purified by flash column chromatography on silica gel (diethyl ether /petroleum ether, 1:10) to obtain product in $71 \%(42.9 \mathrm{mg})$ yield as a yellow oil. ${ }^{1} \mathbf{H}$ NMR (600 $\left.\mathrm{MHz}, \mathrm{CDCl}_{3}\right) \delta 7.85-7.82(\mathrm{~m}, 4 \mathrm{H}), 7.58-7.57(\mathrm{~m}, 1 \mathrm{H}), 7.49-7.45(\mathrm{~m}, 4 \mathrm{H}), 7.32(\mathrm{t}, J=7.8 \mathrm{~Hz}, 2 \mathrm{H})$, $7.23-7.19(\mathrm{~m}, 1 \mathrm{H}), 5.58(\mathrm{~s}, 1 \mathrm{H}), 5.38(\mathrm{~s}, 1 \mathrm{H}), 3.41(\mathrm{q}, J=7.2 \mathrm{~Hz}, 1 \mathrm{H}), 2.06(\mathrm{~s}, 1 \mathrm{H}), 1.33(\mathrm{~s}, 3 \mathrm{H}), 1.07$ $(\mathrm{d}, J=7.2 \mathrm{~Hz}, 3 \mathrm{H}) .{ }^{13} \mathbf{C}$ NMR $\left(150 \mathrm{MHz}, \mathrm{CDCl}_{3}\right) \delta 151.9,147.1,142.2,133.4,132.6,128.1,127.9$, 127.6, 126.3, 126.2, 125.9, 125.1, 124.9, 124.8, 115.9, 76.2, 48.2, 30.8, 16.5. HRMS (ESI-TOF) ( $\mathrm{m} / \mathrm{z}$ ): Calcd for $\mathrm{C}_{22} \mathrm{H}_{22} \mathrm{ONa}\left([\mathrm{M}+\mathrm{Na}]^{+}\right)$: 325.1563 ; found: 325.1570 . Specific rotation $[\alpha]^{15} \mathrm{D}=-47.1,(\mathrm{c}=1.00$, $\mathrm{CHCl}_{3}$ ). HPLC analysis (IA, Hexane/IPA $=98 / 2,0.5 \mathrm{~mL} / \mathrm{min}, 250 \mathrm{~nm}$ ) indicated $92 \%$ ee: $\mathrm{tR}$ (major) $=$ $19.94 \mathrm{~min}, \mathrm{tR}($ minor $)=26.55 \mathrm{~min}$.
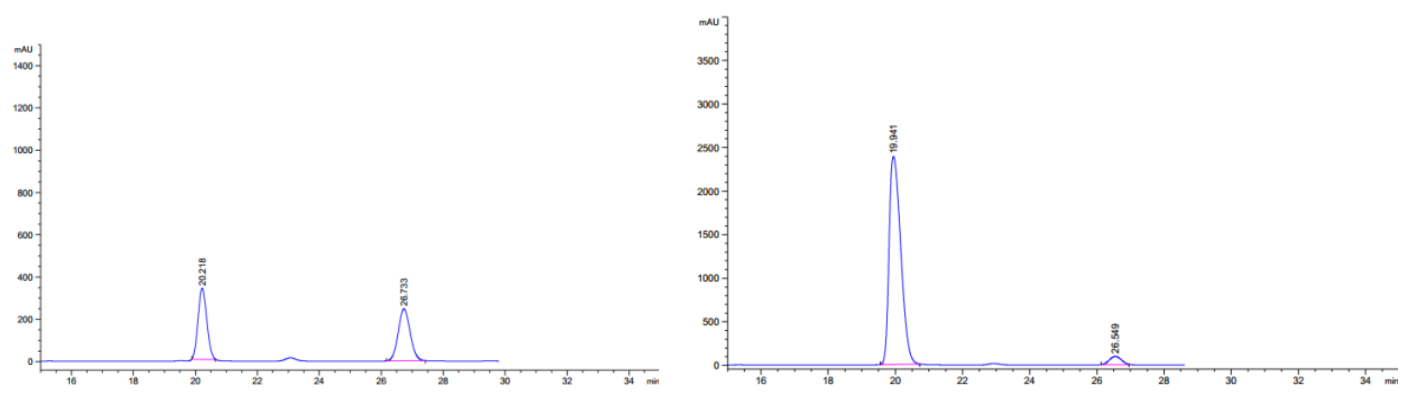


\begin{tabular}{llllc}
\hline $\begin{array}{l}\text { Peak } \\
\#\end{array}$ & $\begin{array}{l}\text { PetTime } \\
{[\mathrm{min}]}\end{array}$ & $\begin{array}{l}\text { Width } \\
{[\mathrm{min}]}\end{array}$ & $\begin{array}{l}\text { Area } \\
{[\mathrm{mAU} * \mathrm{~S}]}\end{array}$ & $\begin{array}{c}\text { Area } \\
\%\end{array}$ \\
\hline 1 & 20.22 & 0.3251 & 6569.70964 & 49.9728 \\
2 & 26.73 & 0.4457 & 6576.86768 & 50.0272 \\
\hline
\end{tabular}

\begin{tabular}{|c|c|c|c|c|}
\hline $\begin{array}{l}\text { Peak } \\
\#\end{array}$ & $\begin{array}{l}\text { PetTime } \\
{[\mathrm{min}]}\end{array}$ & $\begin{array}{l}\text { Width } \\
{[\mathrm{min}]}\end{array}$ & $\begin{array}{l}\text { Area } \\
{\left[\mathrm{mAU}^{*} \mathrm{~S}\right]}\end{array}$ & $\begin{array}{r}\text { Area } \\
\%\end{array}$ \\
\hline 1 & 19.94 & 0.4029 & $5.77096 \mathrm{e} 4$ & 95.9485 \\
\hline 2 & 26.55 & 0.4182 & 2436.80347 & 4.0515 \\
\hline
\end{tabular}

\section{(2S,3R)-4-(4-fluorophenyl)-3-methyl-2-phenylpent-4-en-2-ol}

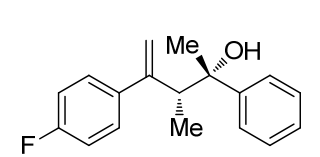

3ae

Prepared according to general procedure. In a nitrogen-filled glovebox, a flamedried screw-cap reaction tube equipped with a Teflon-coated magnetic stir bar were charged with $\mathrm{Cu}(\mathrm{OAc})_{2}(1.8 \mathrm{mg}, 10.0 \mu \mathrm{mol}),(R, R)-\mathrm{Ph}-\mathrm{BPE}(6.1 \mathrm{mg}, 12.0$ $\mu \mathrm{mol})$, and anhydrous THF ( $1 \mathrm{~mL})$, dimethoxymethylsilane (DMMS, 3 equiv) were subsequently added. The resulting mixture was stirred for $2 \mathrm{~min}$ and then 1(buta-1,3-dien-2-yl)-4-fluorobenzene $(59.2 \mathrm{mg}, 0.4 \mathrm{mmol})$ and acetophenone $(24.0 \mathrm{mg}, 0.2 \mathrm{mmol})$ were added, respectively. The vial was removed from the glove box, and the mixture was stirred at $20{ }^{\circ} \mathrm{C}$ for $18 \mathrm{~h}$. After $18 \mathrm{~h}$ the cap was removed. The solution was quenched by adding saturated ammonium fluoride in methanol using a $5 \mathrm{~mL}$ syringe $(3 \mathrm{~mL})$ and stirred for $30 \mathrm{~min}$. After removal of solvent under reduced pressure with aid of a rotary evaporator, the diastereomeric ratio (dr) was assessed by ${ }^{1} \mathrm{H}$ NMR in $\mathrm{CDCl}_{3}$, The resulting crude product was purified by flash column chromatography on silica gel (diethyl ether /petroleum ether, 1:10) to obtain product in $92 \%(49.7 \mathrm{mg})$ yield as a colorless oil. ${ }^{1} \mathbf{H}$ NMR (600 $\left.\mathrm{MHz}, \mathrm{CDCl}_{3}\right) \delta 7.42(\mathrm{~d}, J=7.6 \mathrm{~Hz}, 2 \mathrm{H}), 7.37-7.35(\mathrm{~m}, 2 \mathrm{H}), 7.31(\mathrm{t}, J=7.8 \mathrm{~Hz}, 2 \mathrm{H}), 7.21(\mathrm{t}, J=7.2$ $\mathrm{Hz}, 1 \mathrm{H}), 7.02$ (t, $J=8.4 \mathrm{~Hz}, 2 \mathrm{H}), 5.40$ (s, 1H), 5.25 (s, 1H), 3.20 (q, J=7.2 Hz, 1H), 1.96 (s, 1H), 1.33 $(\mathrm{s}, 3 \mathrm{H}), 1.01(\mathrm{~d}, J=7.2 \mathrm{~Hz}, 3 \mathrm{H}) .{ }^{13} \mathbf{C}$ NMR $(150 \mathrm{MHz}, \mathrm{CDCl} 3) \delta 162.1(\mathrm{~d}, J=246.0 \mathrm{~Hz}), 151.0,147.0$, 140.9 (d, $J=4.5 \mathrm{~Hz}), 128.0,127.94,127.93,126.4,124.9,115.4,115.2$ (d, $J=21.0 \mathrm{~Hz}), 76.1,48.5,30.4$, 16.4. ${ }^{19}$ F NMR (470 MHz, $\left.\mathrm{CDCl}_{3}\right) \delta-115.33--115.54(\mathrm{~m}, 1 \mathrm{~F})$. HRMS (ESI-TOF) (m/z): Calcd for $\mathrm{C}_{18} \mathrm{H}_{19} \mathrm{FONa}\left([\mathrm{M}+\mathrm{Na}]^{+}\right): 293.1318$; found: 293.1322 . Specific rotation $[\alpha]^{15} \mathrm{D}=-23.2,\left(\mathrm{c}=1.00, \mathrm{CHCl}_{3}\right)$. HPLC analysis (IA, Hexane/IPA $=98 / 2,0.5 \mathrm{~mL} / \mathrm{min}, 250 \mathrm{~nm}$ ) indicated $91 \%$ ee: $\mathrm{tR}$ (major) $=16.65$ $\min , \mathrm{tR}($ minor $)=23.50 \mathrm{~min}$.

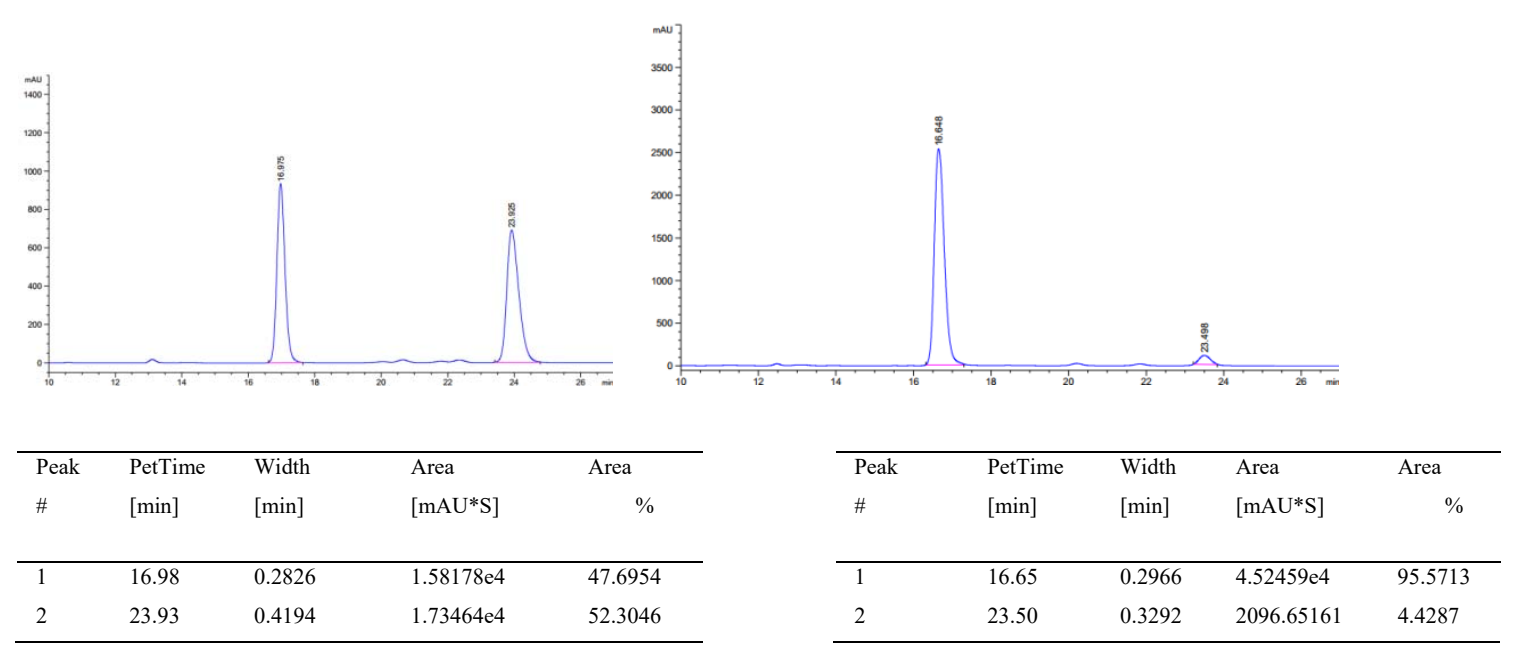




\section{(2S,3R)-4-(4-chlorophenyl)-3-methyl-2-phenylpent-4-en-2-ol}<smiles>C=C(c1ccc(Cl)cc1)C(C)(O)C(C)(O)c1ccccc1</smiles>

3af

Prepared according to general procedure. In a nitrogen-filled glovebox, a flamedried screw-cap reaction tube equipped with a Teflon-coated magnetic stir bar were charged with $\mathrm{Cu}(\mathrm{OAc})_{2}(1.8 \mathrm{mg}, 10.0 \mu \mathrm{mol}),(R, R)-\mathrm{Ph}-\mathrm{BPE}(6.1 \mathrm{mg}, 12.0$ $\mu \mathrm{mol})$, and anhydrous THF ( $1 \mathrm{~mL})$, dimethoxymethylsilane (DMMS, 3 equiv) were subsequently added. The resulting mixture was stirred for $2 \mathrm{~min}$ and then 1-(buta-1,3-dien-2-yl)-4-chlorobenzene $(65.8 \mathrm{mg}, 0.4 \mathrm{mmol})$ and acetophenone (24.0 $\mathrm{mg}, 0.2 \mathrm{mmol})$ were added, respectively. The vial was removed from the glove box, and the mixture was stirred at $20{ }^{\circ} \mathrm{C}$ for $18 \mathrm{~h}$. After $18 \mathrm{~h}$ the cap was removed. The solution was quenched by adding saturated ammonium fluoride in methanol using a $5 \mathrm{~mL}$ syringe $(3 \mathrm{~mL})$ and stirred for $30 \mathrm{~min}$. After removal of solvent under reduced pressure with aid of a rotary evaporator, the diastereomeric ratio (dr) was assessed by ${ }^{1} \mathrm{H}$ NMR in $\mathrm{CDCl}_{3}$, The resulting crude product was purified by flash column chromatography on silica gel (diethyl ether /petroleum ether, $1: 10)$ to obtain product in $82 \%(46.9 \mathrm{mg})$ yield as a yellow oil. ${ }^{1} \mathbf{H}$ NMR (600 $\left.\mathrm{MHz} \mathrm{CDCl}_{3}\right) \delta 7.41(\mathrm{~d}, J=7.8 \mathrm{~Hz}, 2 \mathrm{H}), 7.34-7.30(\mathrm{~m}, 6 \mathrm{H}), 7.21(\mathrm{t}, J=7.2 \mathrm{~Hz}, 1 \mathrm{H}), 5.43(\mathrm{~s}, 1 \mathrm{H}), 5.28$ $(\mathrm{s}, 1 \mathrm{H}), 3.19$ (q, $J=7.2 \mathrm{~Hz}, 1 \mathrm{H}), 1.93(\mathrm{~s}, 1 \mathrm{H}), 1.33(\mathrm{~s}, 3 \mathrm{H}), 1.01(\mathrm{~d}, J=7.2 \mathrm{~Hz}, 3 \mathrm{H}) .{ }^{13} \mathbf{C}$ NMR $(150$ $\left.\mathrm{MHz}, \mathrm{CDCl}_{3}\right) \delta 150.8,146.9,143.3,133.0,128.5,128.0,127.7,126.4,124.9,115.9,76.1,48.3,30.4$, 16.4. HRMS (ESI-TOF) $(\mathrm{m} / \mathrm{z})$ : Calcd for $\mathrm{C}_{18} \mathrm{H}_{19} \mathrm{ClONa}\left([\mathrm{M}+\mathrm{Na}]^{+}\right)$: 309.1025; found: 309.1021 . Specific rotation $[\alpha]^{16} \mathrm{D}=-22.1,\left(\mathrm{c}=1.00, \mathrm{CHCl}_{3}\right)$. HPLC analysis (IA, Hexane $/ \mathrm{IPA}=98 / 2,0.5 \mathrm{~mL} / \mathrm{min}$, $250 \mathrm{~nm}$ ) indicated $90 \%$ ee: $\mathrm{tR}($ major $)=17.88 \mathrm{~min}, \mathrm{tR}($ minor $)=24.61 \mathrm{~min}$.
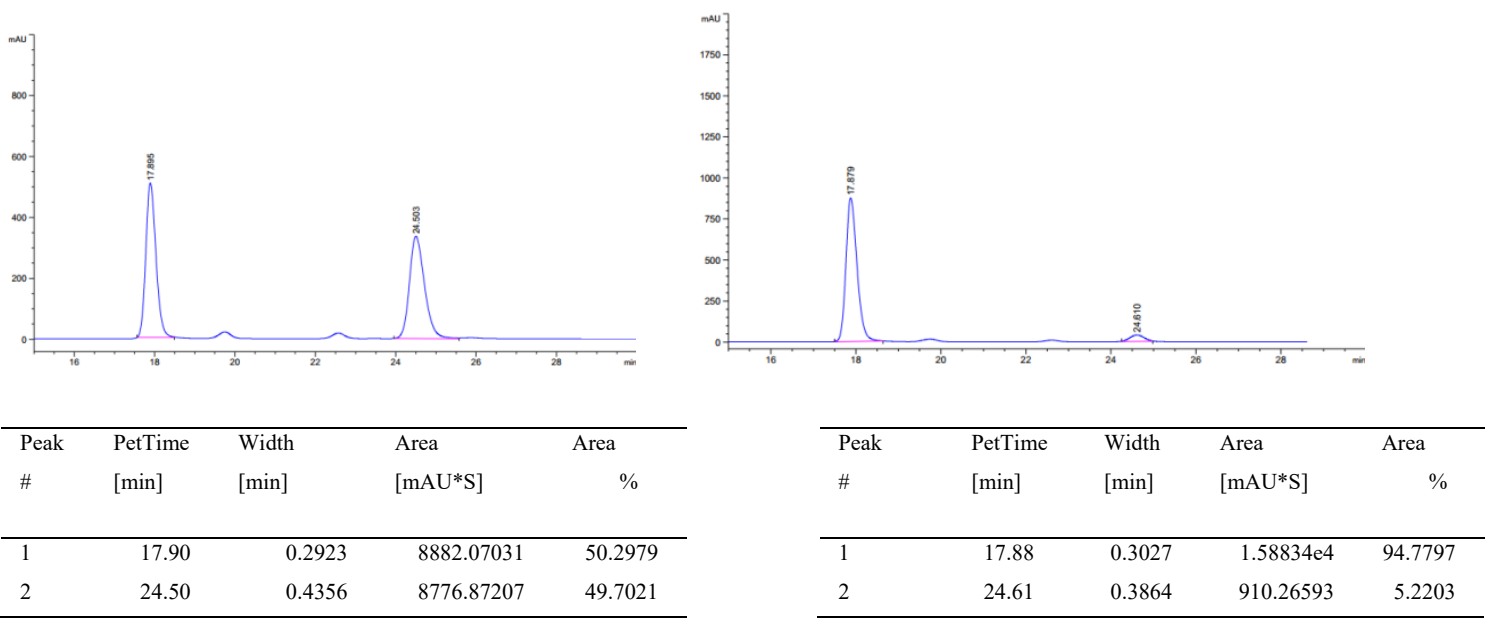

\section{(2S,3R)-3-methyl-2-phenyl-4-(4-(trifluoromethyl)phenyl)pent-4-en-2-ol}

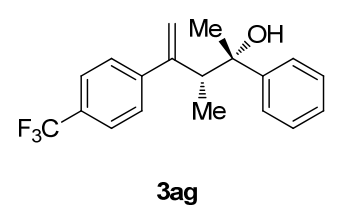

Prepared according to general procedure. In a nitrogen-filled glovebox, a flame-dried screw-cap reaction tube equipped with a Teflon-coated magnetic stir bar were charged with $\mathrm{Cu}(\mathrm{OAc})_{2}(1.8 \mathrm{mg}, 10.0 \mu \mathrm{mol}),(R, R)-\mathrm{Ph}-\mathrm{BPE}(6.1$ $\mathrm{mg}, 12.0 \mu \mathrm{mol})$, and anhydrous THF ( $1 \mathrm{~mL}$ ), dimethoxymethylsilane (DMMS, 3 equiv) were subsequently added. The resulting mixture was stirred for $2 \mathrm{~min}$ and then 1-(buta-1,3-dien-2-yl)-4-(trifluoromethyl)benzene (79.2mg, $0.4 \mathrm{mmol}$ ) and acetophenone (24.0 $\mathrm{mg}, 0.2 \mathrm{mmol}$ ) were added, respectively. The vial was removed from the glove box, and the mixture was stirred at $20{ }^{\circ} \mathrm{C}$ for $18 \mathrm{~h}$. After $18 \mathrm{~h}$ the cap was removed. The solution was quenched by adding 
saturated ammonium fluoride in methanol using a $5 \mathrm{~mL}$ syringe $(3 \mathrm{~mL})$ and stirred for $30 \mathrm{~min}$. After removal of solvent under reduced pressure with aid of a rotary evaporator, the diastereomeric ratio (dr) was assessed by ${ }^{1} \mathrm{H}$ NMR in $\mathrm{CDCl}_{3}$, The resulting crude product was purified by flash column chromatography on silica gel (diethyl ether /petroleum ether, 1:10) to obtain product in $95 \%(60.8 \mathrm{mg})$ yield as a colorless oil. ${ }^{1} \mathbf{H}$ NMR $\left(600 \mathrm{MHz}, \mathrm{CDCl}_{3}\right) \delta 7.57(\mathrm{~d}, J=8.4 \mathrm{~Hz}, 2 \mathrm{H}), 7.48(\mathrm{~d}, J=8.4 \mathrm{~Hz}, 2 \mathrm{H})$, 7.41 (d, $J=7.8 \mathrm{~Hz}, 2 \mathrm{H}), 7.30$ (t, $J=7.8 \mathrm{~Hz}, 2 \mathrm{H}), 7.20$ (t, $J=7.2 \mathrm{~Hz}, 1 \mathrm{H}), 5.47$ (s, $1 \mathrm{H}), 5.35$ (s, 1H), 3.22 (q, $J=7.2 \mathrm{~Hz}, 1 \mathrm{H}), 1.91(\mathrm{~s}, 1 \mathrm{H}), 1.35(\mathrm{~s}, 3 \mathrm{H}), 1.06(\mathrm{~d}, J=7.2 \mathrm{~Hz}, 3 \mathrm{H}) .{ }^{13} \mathbf{C}$ NMR $(150 \mathrm{MHz}, \mathrm{CDCl} 3) \delta$ 150.8, 148.4, 146.8, 129.1 (q, $J=33.0 \mathrm{~Hz}), 128.0,126.7,126.5,125.3$ (q, $J=3.0 \mathrm{~Hz}), 124.9,124.2$ (q, $J$ $=270.0 \mathrm{~Hz}), 117.2,76.2,48.4,30.0,16.4 .{ }^{19} \mathbf{F}$ NMR $\left(470 \mathrm{MHz}, \mathrm{CDCl}_{3}\right) \delta-62.40(\mathrm{~s}, 3 \mathrm{~F})$. HRMS (ESITOF) (m/z): Calcd for $\mathrm{C}_{19} \mathrm{H}_{19} \mathrm{~F}_{3} \mathrm{ONa}\left([\mathrm{M}+\mathrm{Na}]^{+}\right)$: 343.1286 ; found: 343.1288 . Specific rotation $[\alpha]^{15} \mathrm{D}$ $=-31.7,\left(\mathrm{c}=1.00, \mathrm{CHCl}_{3}\right)$. HPLC analysis (IA, Hexane $/ \mathrm{IPA}=98 / 2,0.5 \mathrm{~mL} / \mathrm{min}, 250 \mathrm{~nm}$ ) indicated $90 \%$ ee: $\operatorname{tR}($ major $)=16.06 \mathrm{~min}, \mathrm{tR}($ minor $)=21.14 \mathrm{~min}$.
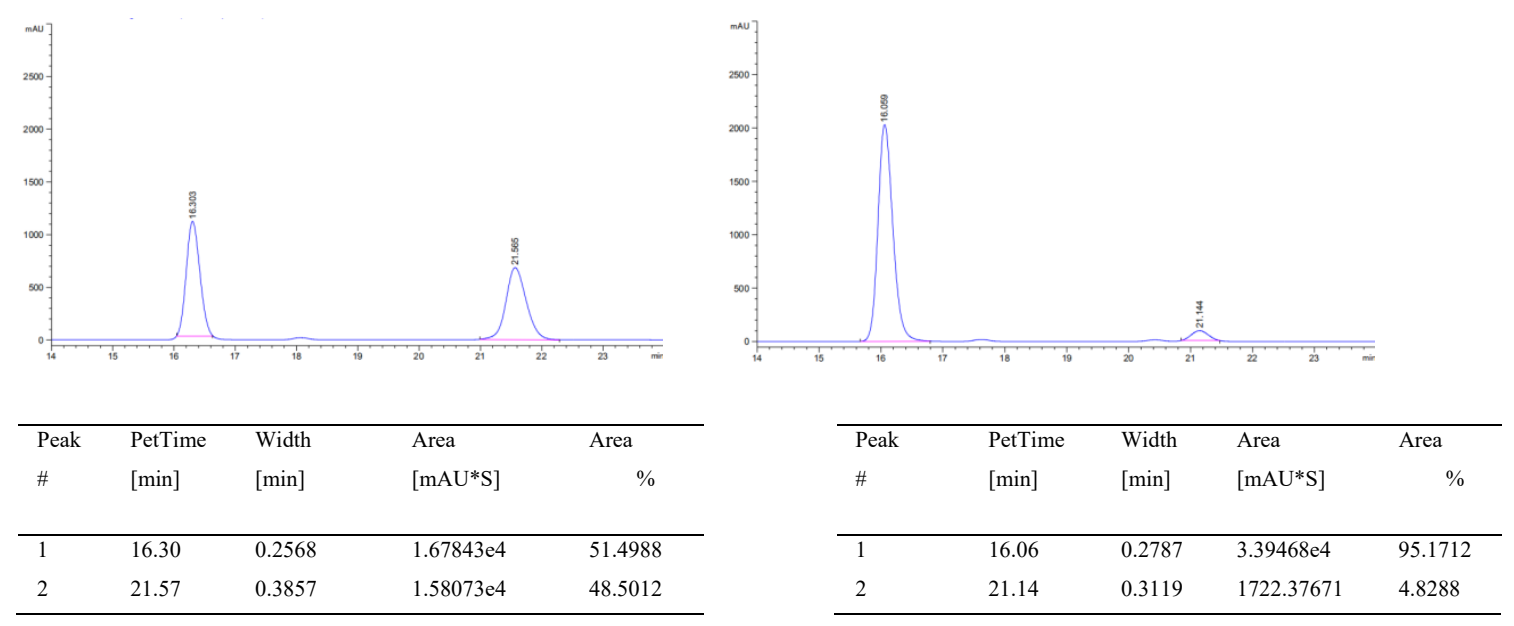

\section{(2S,3R)-4-(6-methoxypyridin-3-yl)-3-methyl-2-phenylpent-4-en-2-ol}

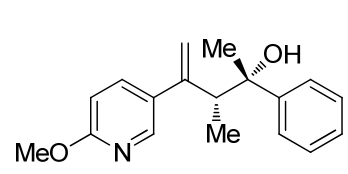

3ah

Prepared according to general procedure. In a nitrogen-filled glovebox, a flame-dried screw-cap reaction tube equipped with a Teflon-coated magnetic stir bar were charged with $\mathrm{Cu}(\mathrm{OAc})_{2}(1.8 \mathrm{mg}, 10.0 \mu \mathrm{mol}),(R, R)-\mathrm{Ph}-\mathrm{BPE}(6.1$ $\mathrm{mg}, 12.0 \mu \mathrm{mol})$, and anhydrous THF $(1 \mathrm{~mL})$, dimethoxymethylsilane (DMMS, 3 equiv) were subsequently added. The resulting mixture was stirred for $2 \mathrm{~min}$ and then 5-(buta-1,3-dien-2-yl)-2-methoxypyridine $(64.5 \mathrm{mg}, 0.4 \mathrm{mmol})$ and acetophenone ( $24.0 \mathrm{mg}, 0.2 \mathrm{mmol})$ were added, respectively. The vial was removed from the glove box, and the mixture was stirred at $20{ }^{\circ} \mathrm{C}$ for $18 \mathrm{~h}$. After $18 \mathrm{~h}$ the cap was removed. The solution was quenched by adding saturated ammonium fluoride in methanol using a $5 \mathrm{~mL}$ syringe $(3 \mathrm{~mL})$ and stirred for $30 \mathrm{~min}$. After removal of solvent under reduced pressure with aid of a rotary evaporator, the diastereomeric ratio (dr) was assessed by ${ }^{1} \mathrm{H} N M R$ in $\mathrm{CDCl}_{3}$, The resulting crude product was purified by flash column chromatography on silica gel (diethyl ether /petroleum ether, 1:10) to obtain product in 93\% (52.6 mg) yield as a yellow oil. ${ }^{1} \mathbf{H}$ NMR $\left(600 \mathrm{MHz}, \mathrm{CDCl}_{3}\right) \delta 8.21(\mathrm{~d}, J=2.4 \mathrm{~Hz}, 1 \mathrm{H}), 7.62-7.60$ (m, 1H), $7.42(\mathrm{~d}, J=7.8 \mathrm{~Hz}, 2 \mathrm{H}), 7.31(\mathrm{t}, J=7.2 \mathrm{~Hz}, 2 \mathrm{H}), 7.21(\mathrm{t}, J=7.2 \mathrm{~Hz}, 1 \mathrm{H}), 6.71(\mathrm{~d}, J=8.4 \mathrm{~Hz}$, $1 \mathrm{H}), 5.40(\mathrm{~s}, 1 \mathrm{H}), 5.25(\mathrm{~s}, 1 \mathrm{H}), 3.95(\mathrm{~s}, 3 \mathrm{H}), 3.15(\mathrm{q}, J=7.2 \mathrm{~Hz}, 1 \mathrm{H}), 1.96(\mathrm{~s}, 1 \mathrm{H}), 1.37(\mathrm{~s}, 3 \mathrm{H}), 1.02(\mathrm{~d}$, $J=7.2 \mathrm{~Hz}, 3 \mathrm{H}) .{ }^{13} \mathbf{C}$ NMR $\left(150 \mathrm{MHz}, \mathrm{CDCl}_{3}\right) \delta 163.4,148.5,146.9,144.4,136.8,133.4,128.0,126.4$, 124.9, 115.2, 110.3, 76.1, 53.5, 48.2, 30.2, 16.2. HRMS (ESI-TOF) (m/z): Calcd for $\mathrm{C}_{18} \mathrm{H}_{21} \mathrm{NO}_{2} \mathrm{Na}$ ([M $\left.+\mathrm{Na}]^{+}\right)$: 306.1470; found: 306.1465 . Specific rotation $[\alpha]^{15} \mathrm{D}=-33.3,\left(\mathrm{c}=1.00, \mathrm{CHCl}_{3}\right)$. HPLC analysis 
$(\mathrm{IA}$, Hexane $/ \mathrm{IPA}=98 / 2,0.5 \mathrm{~mL} / \mathrm{min}, 250 \mathrm{~nm})$ indicated $78 \%$ ee: $\mathrm{tR}($ major $)=25.94 \mathrm{~min}$, $\mathrm{tR}($ minor $)=$ $31.96 \mathrm{~min}$.

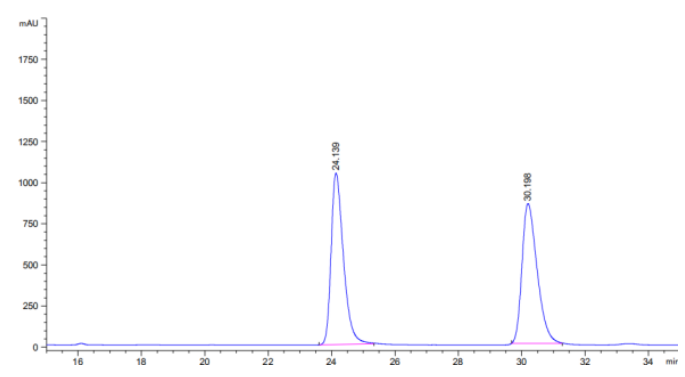

\begin{tabular}{llllc}
\hline $\begin{array}{l}\text { Peak } \\
\#\end{array}$ & $\begin{array}{l}\text { PetTime } \\
{[\mathrm{min}]}\end{array}$ & $\begin{array}{c}\text { Width } \\
{[\mathrm{min}]}\end{array}$ & $\begin{array}{l}\text { Area } \\
{[\mathrm{mAU} * \mathrm{~S}]}\end{array}$ & $\begin{array}{c}\text { Area } \\
\%\end{array}$ \\
\hline 1 & 24.14 & 0.4493 & $2.81027 \mathrm{e} 4$ & 49.9233 \\
2 & 30.20 & 0.5529 & $2.81891 \mathrm{e} 4$ & 50.0767 \\
\hline
\end{tabular}

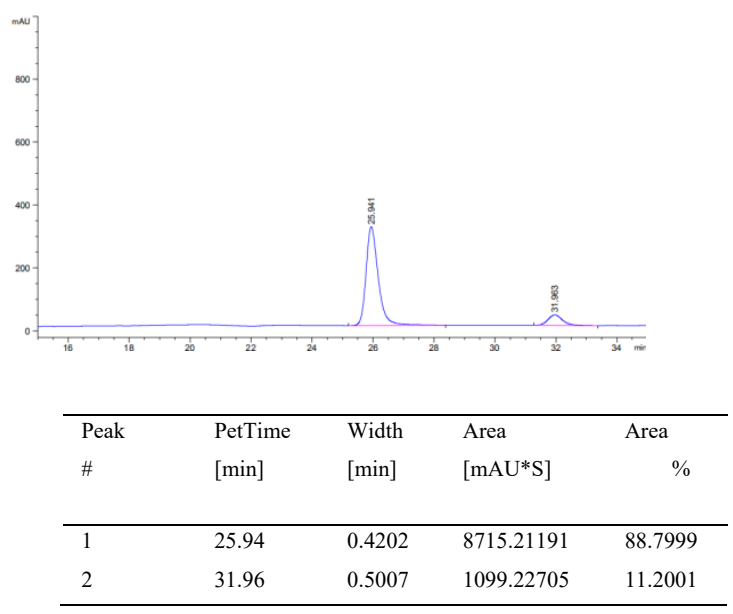

\section{3,4-dimethyl-2-phenylpent-4-en-2-ol}

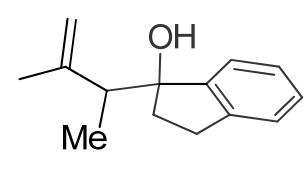

3ai

Prepared according to general procedure. In a nitrogen-filled glovebox, a flamedried screw-cap reaction tube equipped with a Teflon-coated magnetic stir bar were charged with $\mathrm{Cu}(\mathrm{OAc})_{2}(1.8 \mathrm{mg}, 10.0 \mu \mathrm{mol}),(R, R)-\mathrm{Ph}-\mathrm{BPE}(6.1 \mathrm{mg}, 12.0$ $\mu \mathrm{mol})$, and anhydrous THF ( $1 \mathrm{~mL})$, dimethoxymethylsilane (DMMS, 3 equiv) were subsequently added. The resulting mixture was stirred for $2 \mathrm{~min}$ and then isoprene $(27.2 \mathrm{mg}, 0.4 \mathrm{mmol})$ and 2,3-dihydro-1H-inden-1-one $(26.4 \mathrm{mg}, 0.2 \mathrm{mmol})$ were added, respectively. The vial was removed from the glove box, and the mixture was stirred at $20{ }^{\circ} \mathrm{C}$ for $18 \mathrm{~h}$. After $18 \mathrm{~h}$ the cap was removed. The solution was quenched by adding saturated ammonium fluoride in methanol using a $5 \mathrm{~mL}$ syringe $(3 \mathrm{~mL})$ and stirred for $30 \mathrm{~min}$. After removal of solvent under reduced pressure with aid of a rotary evaporator, the diastereomeric ratio (dr) was assessed by ${ }^{1} \mathrm{H} \mathrm{NMR} \mathrm{in} \mathrm{CDCl}_{3}$, The resulting crude product was purified by flash column chromatography on silica gel (diethyl ether /petroleum ether, 1:10) to obtain product (as a mixture of diastereomers, dr: 8:1) in 58\% (23.4 mg) yield as a colorless oil. Major diastereomer: ${ }^{1} \mathbf{H}$ NMR $\left(600 \mathrm{MHz}, \mathrm{CDCl}_{3}\right) \delta 7.35(\mathrm{~d}, J=6.6 \mathrm{~Hz}, 1 \mathrm{H}), 7.24-$ $7.19(\mathrm{~m}, 3 \mathrm{H}), 4.84(\mathrm{~s}, 1 \mathrm{H}), 4.78(\mathrm{~s}, 1 \mathrm{H}), 2.90-2.85(\mathrm{~m}, 1 \mathrm{H}), 2.83-2.76(\mathrm{~m}, 1 \mathrm{H}), 2.59-2.55(\mathrm{~m}, 1 \mathrm{H})$, $2.54-2.51(\mathrm{~m}, 1 \mathrm{H}), 1.98-1.93(\mathrm{~m}, 2 \mathrm{H}), 1.66(\mathrm{~s}, 3 \mathrm{H}), 1.19(\mathrm{~d}, J=7.2 \mathrm{~Hz}, 3 \mathrm{H})$. Major diastereomer : ${ }^{13} \mathbf{C}$ NMR $\left(150 \mathrm{MHz}, \mathrm{CDCl}_{3}\right) \delta 148.2,147.5,142.8,127.9,126.1,124.8,124.1,112.9,85.2,48.3,39.3,29.8$, 22.3, 14.1. HRMS (ESI-TOF) $(\mathrm{m} / \mathrm{z})$ : Calcd for $\mathrm{C}_{14} \mathrm{H}_{18} \mathrm{ONa}\left([\mathrm{M}+\mathrm{Na}]^{+}\right)$: 225.1255; found: 225.1257 .
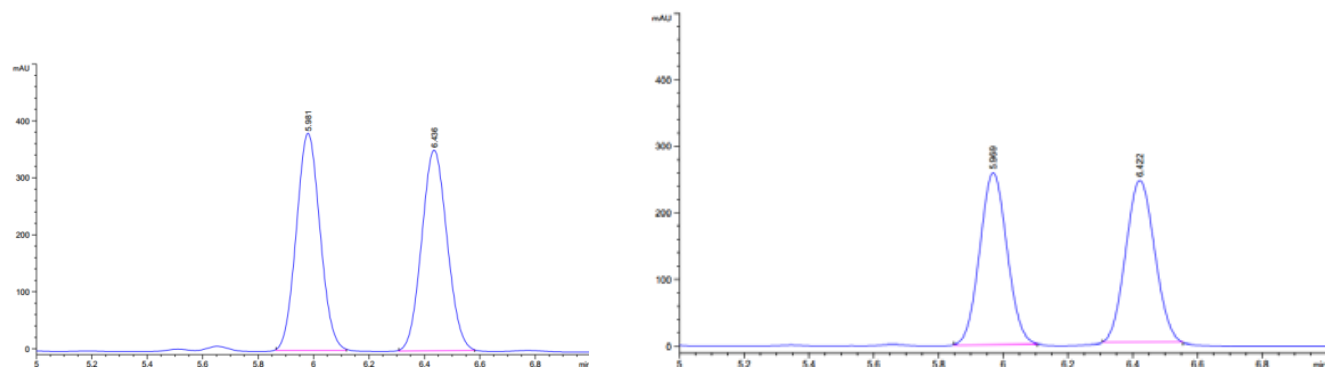


\begin{tabular}{lllll}
\hline $\begin{array}{l}\text { Peak } \\
\#\end{array}$ & $\begin{array}{l}\text { PetTime } \\
{[\mathrm{min}]}\end{array}$ & $\begin{array}{l}\text { Width } \\
{[\mathrm{min}]}\end{array}$ & $\begin{array}{l}\text { Area } \\
{[\mathrm{mAU} * \mathrm{~S}]}\end{array}$ & $\begin{array}{c}\text { Area } \\
\%\end{array}$ \\
\hline 1 & 5.98 & 0.0990 & 380.96674 & 50.1697 \\
2 & 6.43 & 0.1063 & 352.31696 & 49.8303 \\
\hline
\end{tabular}

\begin{tabular}{lllll}
\hline $\begin{array}{l}\text { Peak } \\
\#\end{array}$ & $\begin{array}{l}\text { PetTime } \\
{[\mathrm{min}]}\end{array}$ & $\begin{array}{l}\text { Width } \\
{[\mathrm{min}]}\end{array}$ & $\begin{array}{l}\text { Area } \\
{[\mathrm{mAU} * \mathrm{~S}]}\end{array}$ & $\begin{array}{c}\text { Area } \\
\%\end{array}$ \\
\hline 1 & 5.97 & 0.0977 & 258.30002 & 49.9722 \\
2 & 6.42 & 0.1037 & 243.76846 & 50.0278 \\
\hline
\end{tabular}

\section{(S)-1-((R)-cyclohex-2-en-1-yl)-1-phenylethanol}

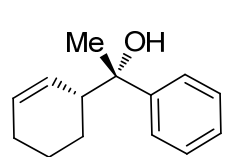

3aj

Prepared according to general procedure. In a nitrogen-filled glovebox, a flame-dried screw-cap reaction tube equipped with a Teflon-coated magnetic stir bar were charged with $\mathrm{Cu}(\mathrm{OAc})_{2}(1.8 \mathrm{mg}, 10.0 \mu \mathrm{mol}),(R, R)-\mathrm{Ph}-\mathrm{BPE}(6.1 \mathrm{mg}, 12.0 \mu \mathrm{mol})$, and anhydrous THF ( $1 \mathrm{~mL})$, dimethoxymethylsilane (DMMS, 3 equiv) were subsequently added. The resulting mixture was stirred for $2 \mathrm{~min}$ and then cyclohexa-1,3-diene (32.0 $\mathrm{mg}, 0.4 \mathrm{mmol})$ and acetophenone $(24.0 \mathrm{mg}, 0.2 \mathrm{mmol})$ were added, respectively. The vial was removed from the glove box, and the mixture was stirred at $20{ }^{\circ} \mathrm{C}$ for $18 \mathrm{~h}$. After $18 \mathrm{~h}$ the cap was removed. The solution was quenched by adding saturated ammonium fluoride in methanol using a $5 \mathrm{~mL}$ syringe $(3 \mathrm{~mL})$ and stirred for $30 \mathrm{~min}$. After removal of solvent under reduced pressure with aid of a rotary evaporator, the diastereomeric ratio (dr) was assessed by ${ }^{1} \mathrm{H} \mathrm{NMR}$ in $\mathrm{CDCl}_{3}$, The resulting crude product was purified by flash column chromatography on silica gel (diethyl ether /petroleum ether, 1:10) to obtain product in $70 \%(28.3 \mathrm{mg})$ yield as a colorless oil. ${ }^{1} \mathbf{H}$ NMR $\left(600 \mathrm{MHz}, \mathrm{CDCl}_{3}\right) \delta 7.42(\mathrm{~d}, J=7.2 \mathrm{~Hz}, 2 \mathrm{H}), 7.34-$ $7.32(\mathrm{~m}, 2 \mathrm{H}), 7.24-7.22(\mathrm{~m}, 1 \mathrm{H}), 5.93-5.90(\mathrm{~m}, 1 \mathrm{H}), 5.78(\mathrm{~d}, J=10.2 \mathrm{~Hz}, 1 \mathrm{H}), 2.56-2.53(\mathrm{~m}, 1 \mathrm{H})$, $1.95-1.94(\mathrm{~m}, 2 \mathrm{H}), 1.75(\mathrm{~s}, 1 \mathrm{H}), 1.73-1.69(\mathrm{~m}, 1 \mathrm{H}), 1.60(\mathrm{~s}, 3 \mathrm{H}), 1.43-1.41(\mathrm{~m}, 2 \mathrm{H}), 1.29-1.25(\mathrm{~m}$, 1H). ${ }^{13} \mathrm{C}$ NMR $\left(150 \mathrm{MHz}, \mathrm{CDCl}_{3}\right) \delta 147.2,131.6,127.9,126.39,126.37,125.2,76.0,46.5,27.9,25.2$, 24.3, 21.9. HRMS (ESI-TOF) $(\mathrm{m} / \mathrm{z})$ : Calcd for $\mathrm{C}_{14} \mathrm{H}_{18} \mathrm{ONa}\left([\mathrm{M}+\mathrm{Na}]^{+}\right)$: 225.1255; found: 225.1254 . Specific rotation $[\alpha]^{15}=-40.3,\left(c=1.00, \mathrm{CHCl}_{3}\right)$. HPLC analysis ( IC, Hexane $/ \mathrm{IPA}=97 / 3,0.8 \mathrm{~mL} / \mathrm{min}$, $250 \mathrm{~nm}$ ) indicated $96 \%$ ee: $\mathrm{tR}$ (major) $=8.40 \mathrm{~min}, \mathrm{tR}($ minor $)=7.87 \mathrm{~min}$.
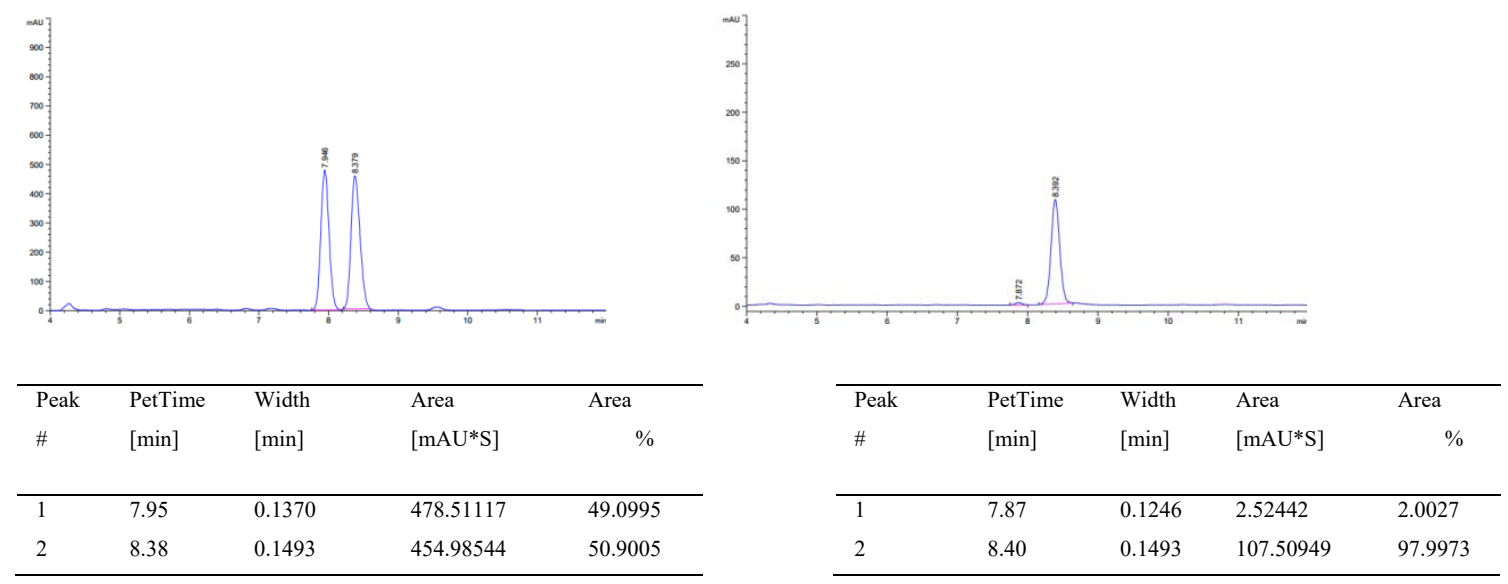

\section{(R)-2-(cyclohex-2-en-1-yl)-2,3-dihydro-1H-inden-2-ol}

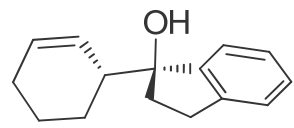

3ak

Prepared according to general procedure. In a nitrogen-filled glovebox, a flamedried screw-cap reaction tube equipped with a Teflon-coated magnetic stir bar were charged with $\mathrm{Cu}(\mathrm{OAc})_{2}(1.8 \mathrm{mg}, 10.0 \mu \mathrm{mol}),(R, R)-\mathrm{Ph}-\mathrm{BPE}(6.1 \mathrm{mg}, 12.0$ $\mu \mathrm{mol})$, and anhydrous THF ( $1 \mathrm{~mL})$, dimethoxymethylsilane (DMMS, 3 equiv) were subsequently added. The resulting mixture was stirred for $2 \mathrm{~min}$ and then cyclohexa-1,3-diene (32.0 mg, $0.4 \mathrm{mmol}$ ) and 2,3-dihydro-1H-inden-1-one (26.4 mg, $0.2 \mathrm{mmol})$ were 
added, respectively. The vial was removed from the glove box, and the mixture was stirred at $20{ }^{\circ} \mathrm{C}$ for $18 \mathrm{~h}$. After $18 \mathrm{~h}$ the cap was removed. The solution was quenched by adding saturated ammonium fluoride in methanol using a $5 \mathrm{~mL}$ syringe $(3 \mathrm{~mL})$ and stirred for $30 \mathrm{~min}$. After removal of solvent under reduced pressure with aid of a rotary evaporator, the diastereomeric ratio (dr) was assessed by ${ }^{1} \mathrm{H}$ NMR in $\mathrm{CDCl}_{3}$, The resulting crude product was purified by flash column chromatography on silica gel (diethyl ether /petroleum ether, 1:10) to obtain product in $64 \%\left(27.4 \mathrm{mg}\right.$ ) yield as a colorless oil. ${ }^{1} \mathbf{H}$ NMR (600 $\left.\mathrm{MHz}, \mathrm{CDCl}_{3}\right) \delta 7.34-7.33(\mathrm{~m}, 1 \mathrm{H}), 7.25-7.23(\mathrm{~m}, 3 \mathrm{H}), 5.77-5.74(\mathrm{~m}, 1 \mathrm{H}), 5.34(\mathrm{~d}, J=10.2 \mathrm{~Hz}, 1 \mathrm{H})$, $3.02-2.98(\mathrm{~m}, 1 \mathrm{H}), 2.82-2.77(\mathrm{~m}, 1 \mathrm{H}), 2.75-2.74(\mathrm{~m}, 1 \mathrm{H}), 2.38-2.34(\mathrm{~m}, 1 \mathrm{H}), 2.04-2.02(\mathrm{~m}, 1 \mathrm{H})$, 2.00- $1.96(\mathrm{~m}, 3 \mathrm{H}), 1.85-1.81(\mathrm{~m}, 2 \mathrm{H}), 1.57-1.50(\mathrm{~m}, 1 \mathrm{H}), 1.45-1.38(\mathrm{~m}, 1 \mathrm{H}) .{ }^{13} \mathbf{C}$ NMR $(150 \mathrm{MHz}$, $\left.\mathrm{CDCl}_{3}\right) \delta 146.2,143.9,129.9,128.3,127.7,126.6,124.9,123.5,86.2,44.2,36.7,29.7,25.2,24.3,22.0$. HRMS (ESI-TOF) $(\mathrm{m} / \mathrm{z})$ : Calcd for $\mathrm{C}_{15} \mathrm{H}_{18} \mathrm{ONa}\left([\mathrm{M}+\mathrm{Na}]^{+}\right)$: 237.1255; found: 237.1257. Specific rotation $[\alpha]^{15} \mathrm{D}=-26.2,\left(\mathrm{c}=1.00, \mathrm{CHCl}_{3}\right) . \mathrm{HPLC}$ analysis $(\mathrm{IC}, \mathrm{Hexane} / \mathrm{IPA}=97 / 3,0.8 \mathrm{~mL} / \mathrm{min}, 250 \mathrm{~nm})$ indicated $92 \%$ ee: $\mathrm{tR}$ (major) $=9.47 \mathrm{~min}, \mathrm{tR}($ minor $)=9.03 \mathrm{~min}$.

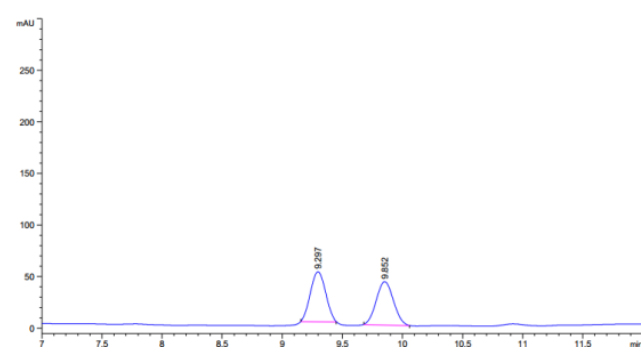

\begin{tabular}{llllc}
\hline $\begin{array}{l}\text { Peak } \\
\#\end{array}$ & $\begin{array}{l}\text { PetTime } \\
{[\mathrm{min}]}\end{array}$ & $\begin{array}{l}\text { Width } \\
{[\mathrm{min}]}\end{array}$ & $\begin{array}{l}\text { Area } \\
{[\mathrm{mAU} * \mathrm{~S}]}\end{array}$ & $\begin{array}{c}\text { Area } \\
\%\end{array}$ \\
\hline 1 & 9.30 & 0.1466 & 48.45168 & 50.2452 \\
2 & 9.85 & 0.1639 & 42.23409 & 49.7548 \\
\hline
\end{tabular}

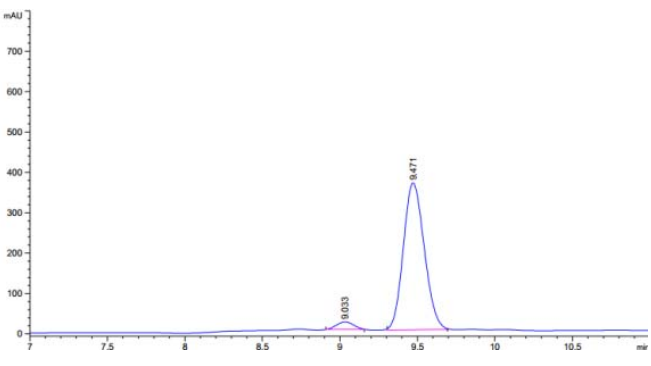

\begin{tabular}{lllll}
\hline $\begin{array}{l}\text { Peak } \\
\#\end{array}$ & $\begin{array}{l}\text { PetTime } \\
{[\mathrm{min}]}\end{array}$ & $\begin{array}{l}\text { Width } \\
{[\mathrm{min}]}\end{array}$ & $\begin{array}{l}\text { Area } \\
{\left[\mathrm{mAU}^{*} \mathrm{~S}\right]}\end{array}$ & $\begin{array}{c}\text { Area } \\
\%\end{array}$ \\
\hline 1 & 9.03 & 0.1279 & 18.42277 & 3.9725 \\
2 & 9.47 & 0.1566 & 363.78043 & 96.0275 \\
\hline
\end{tabular}




\section{V. ${ }^{1} \mathrm{H} 、{ }^{13} \mathrm{C}$ and ${ }^{19} \mathrm{~F}$ Spectra of new compounds}

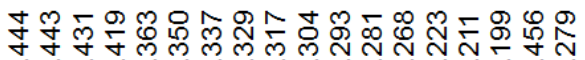

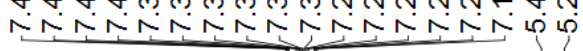

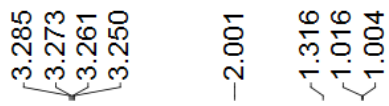

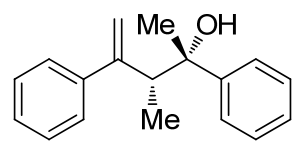

$3 a$

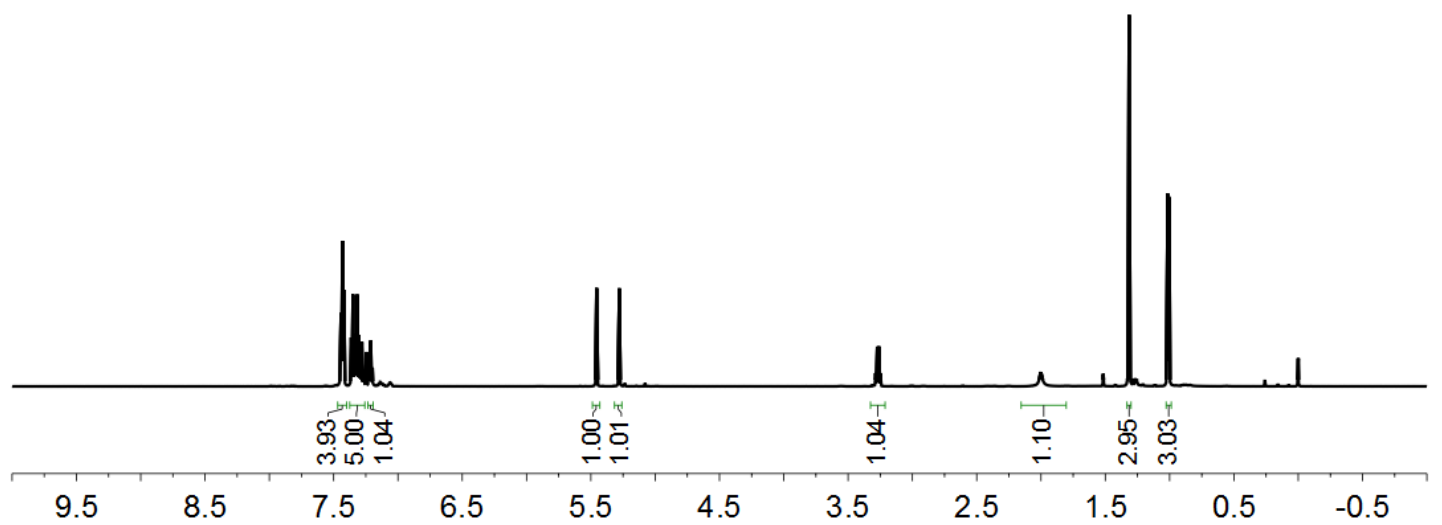

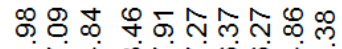

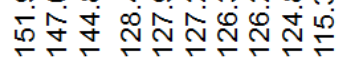

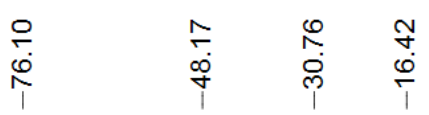

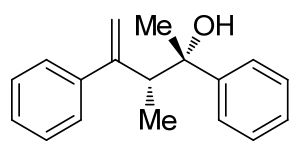

$3 a$

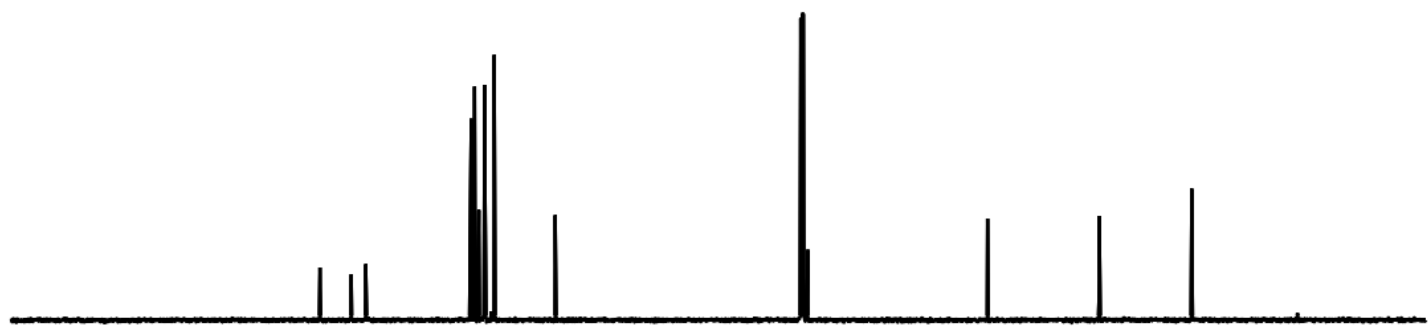

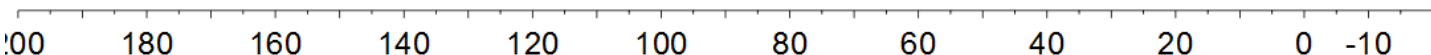




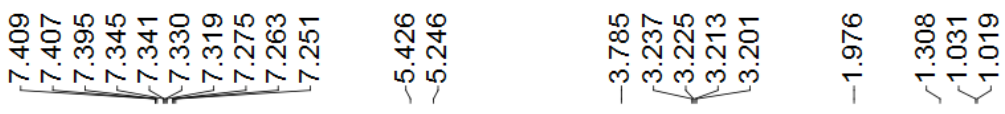

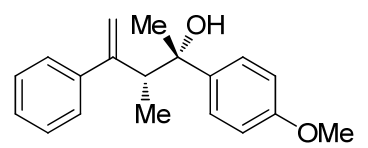

3b

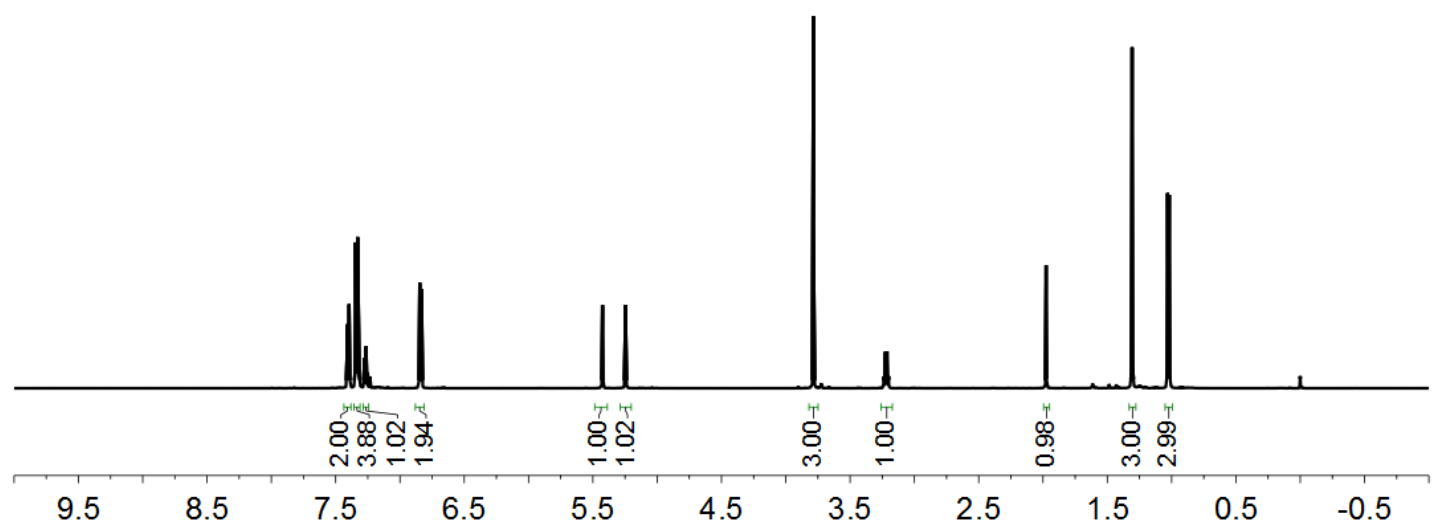

乐

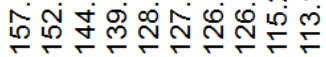

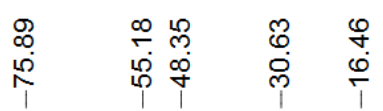

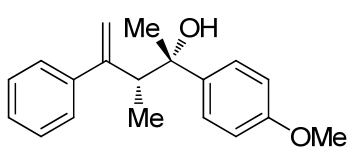

$3 \mathbf{b}$

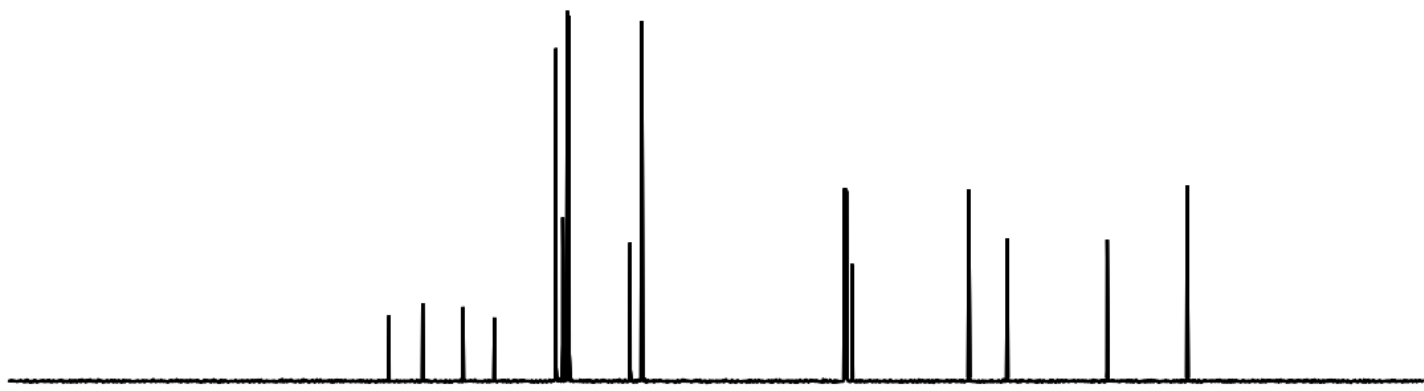




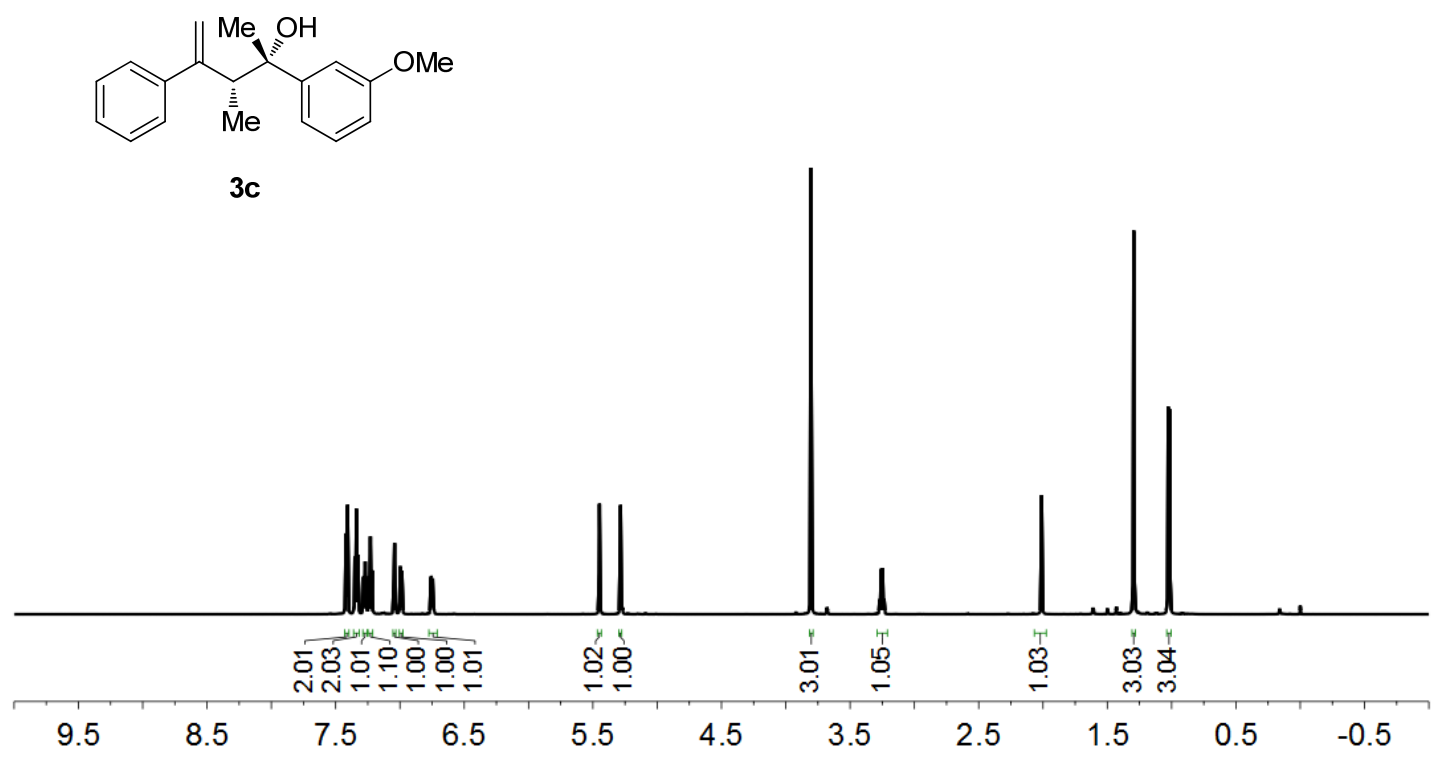

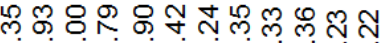

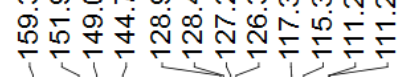

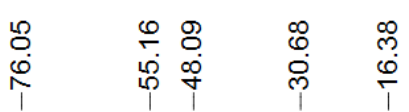

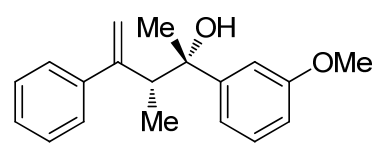

3c

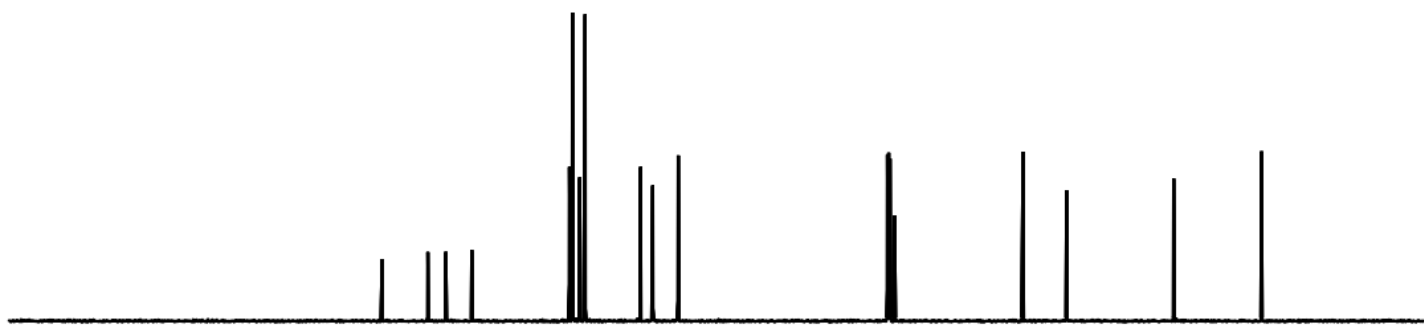

200

170

140

80

60

40

$20 \quad 0$ 


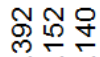

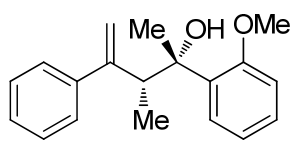

3d

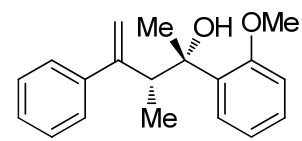

3d

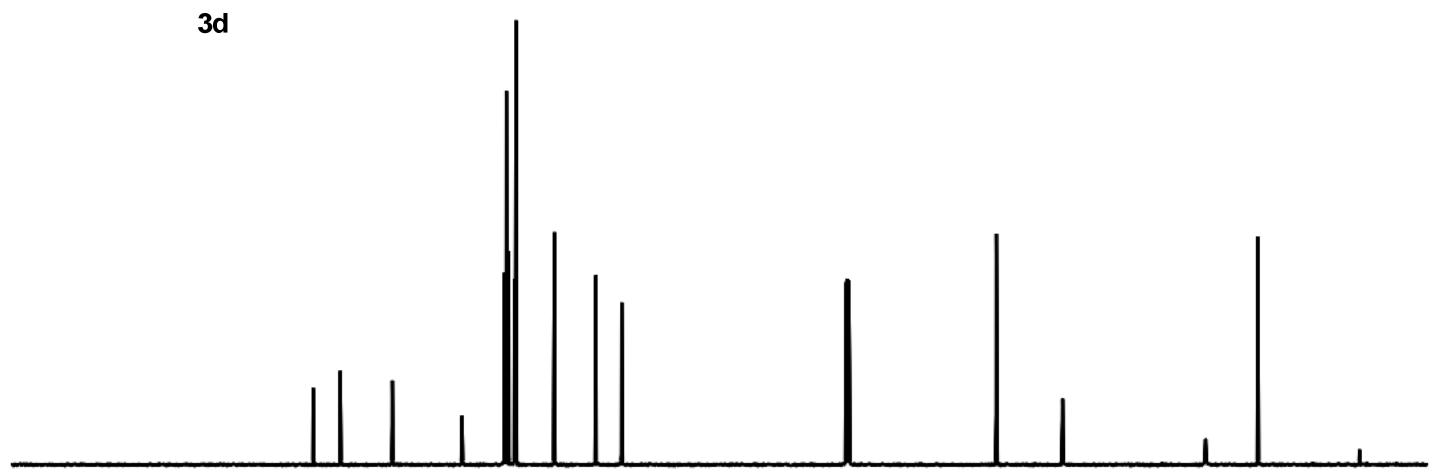

$: 00$ 


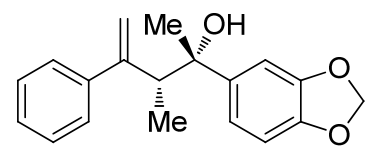

$3 e$

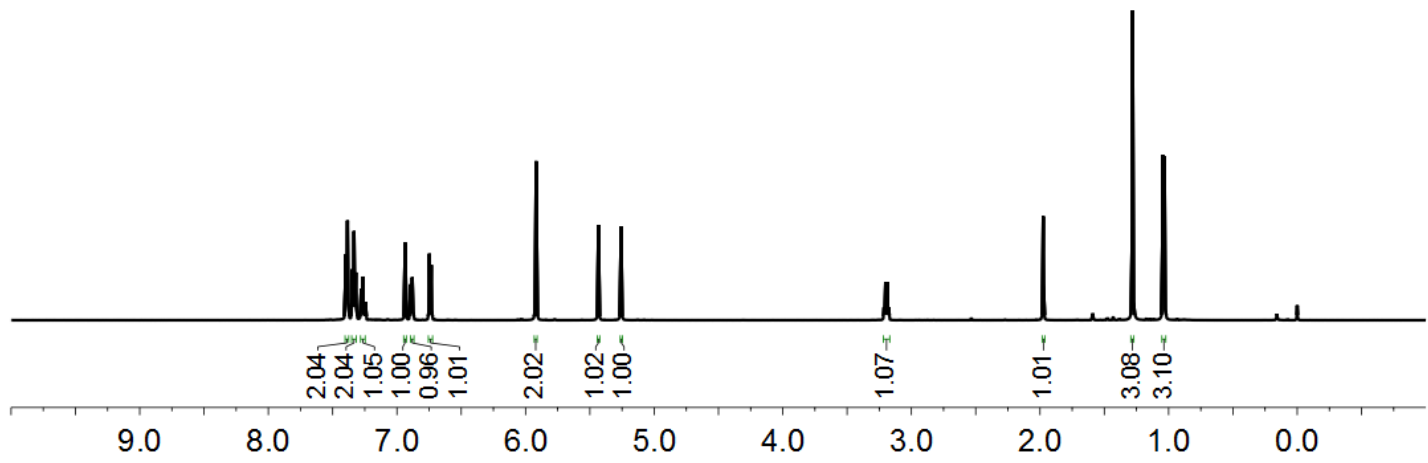

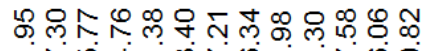

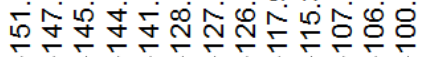
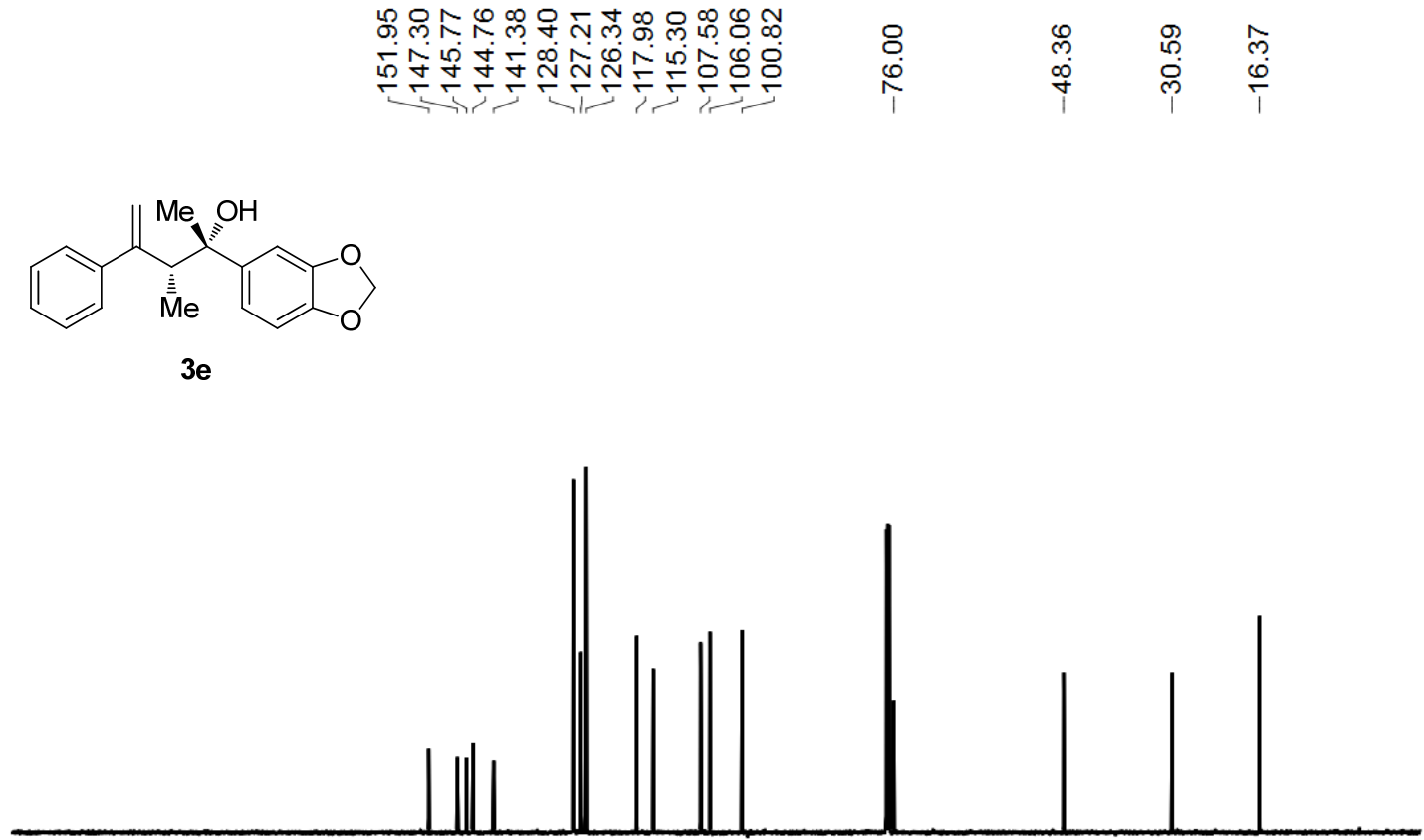

200

170

140

110

80

60

40

20

0 

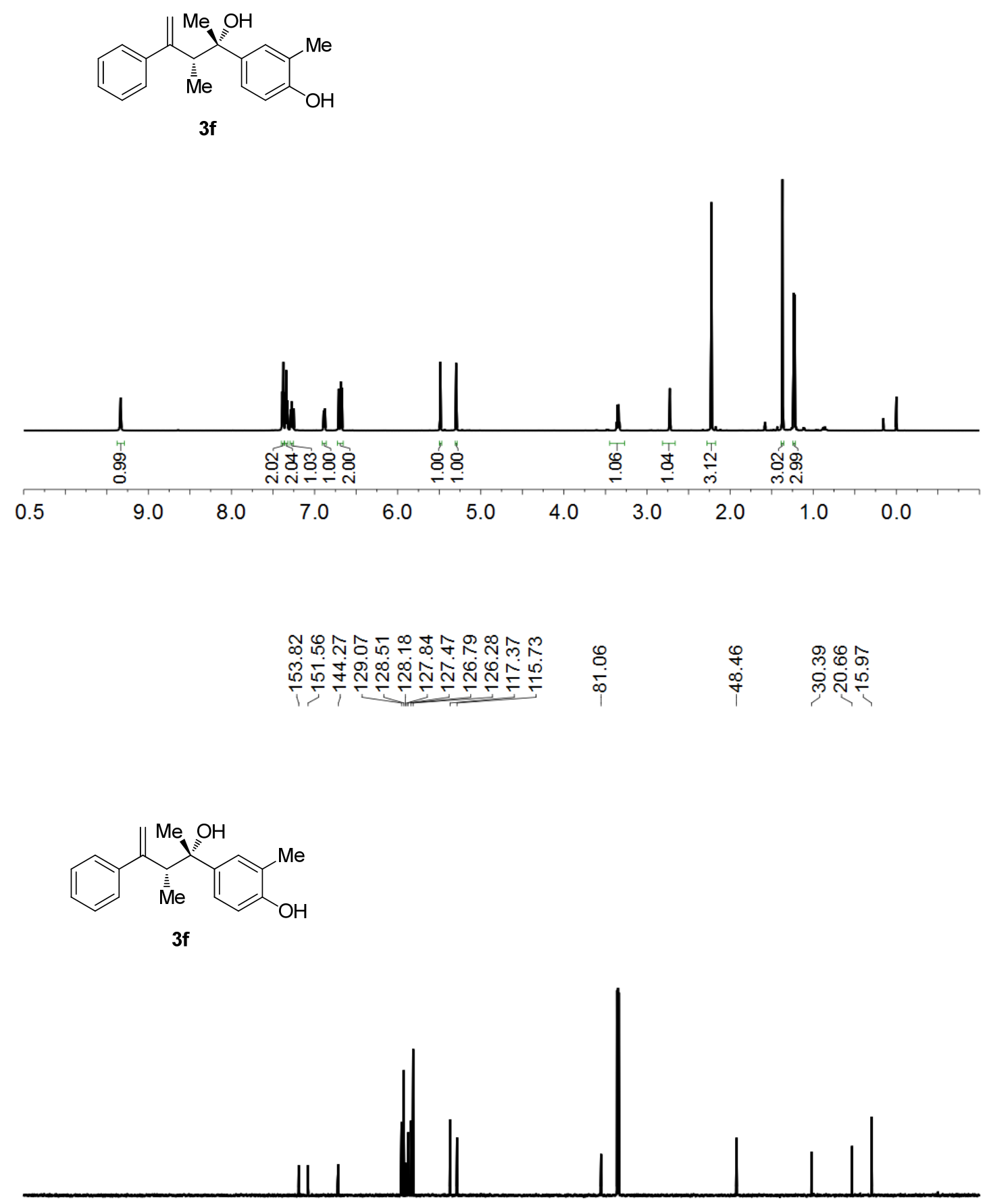


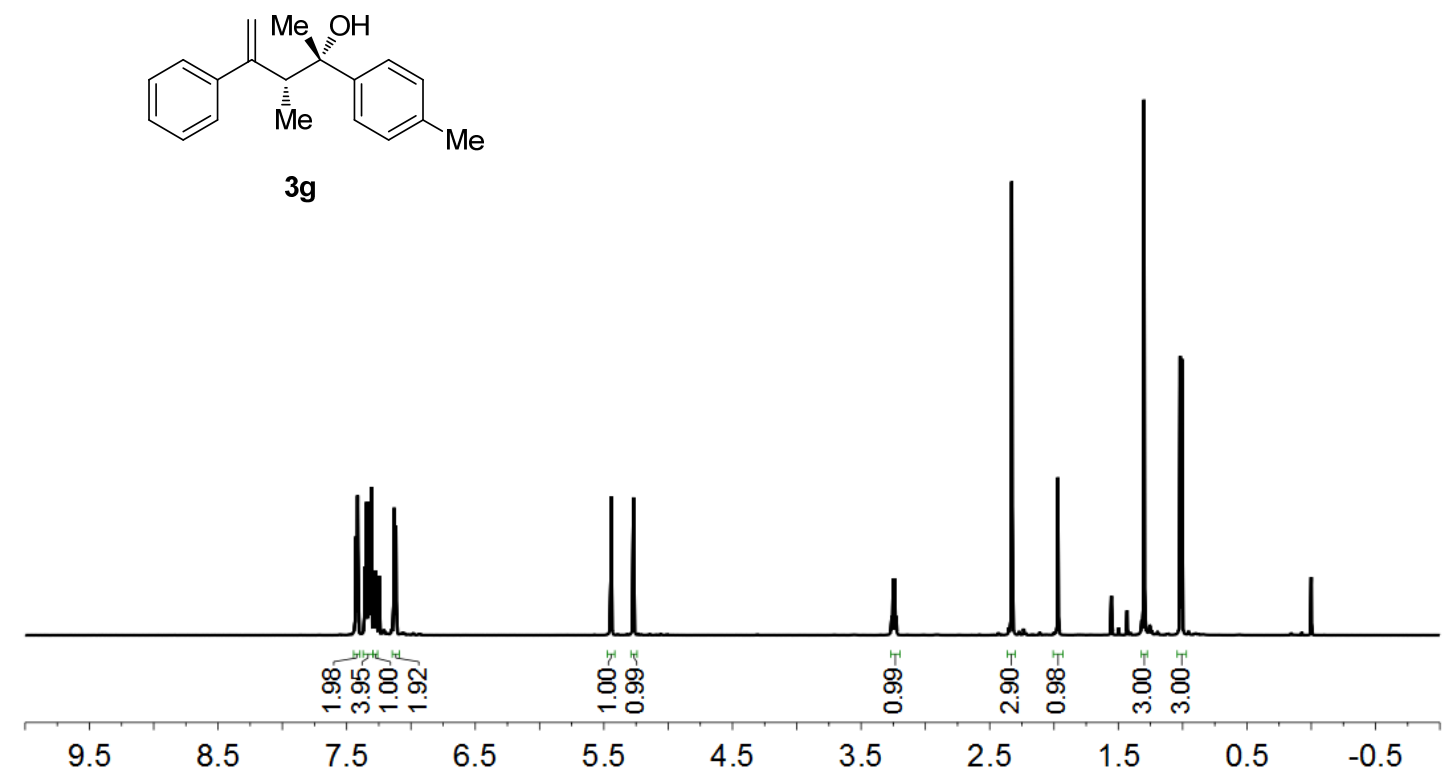

$3 g$

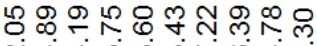

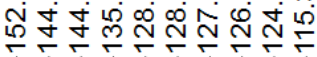

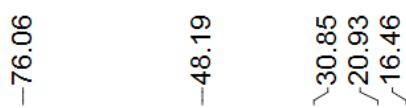

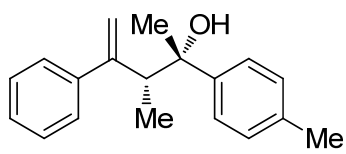

$3 g$

e
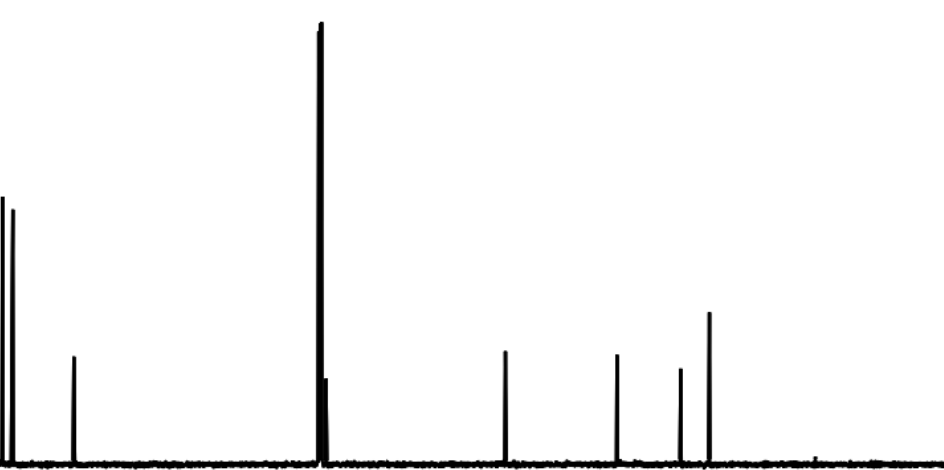

$! 00$

$180 \quad 160$

140

120

100

80

60

40

20

$\begin{array}{ll}0 & -10\end{array}$ 


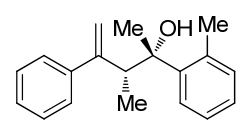

3h

diastereomer

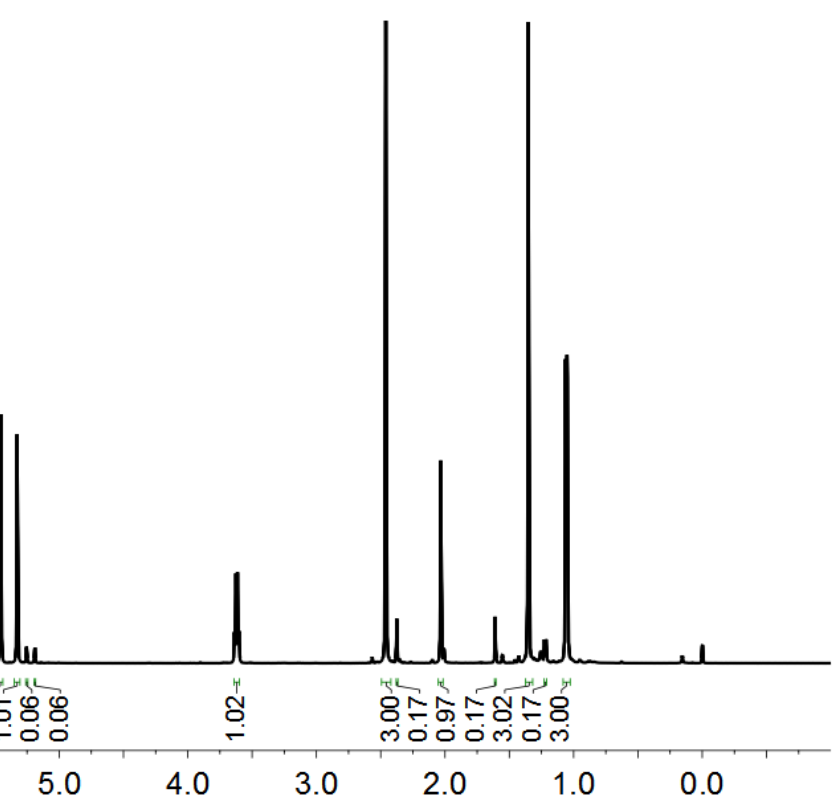

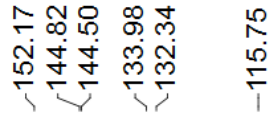

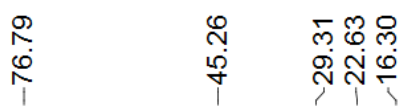

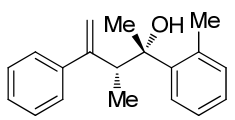

diastereomer

3h

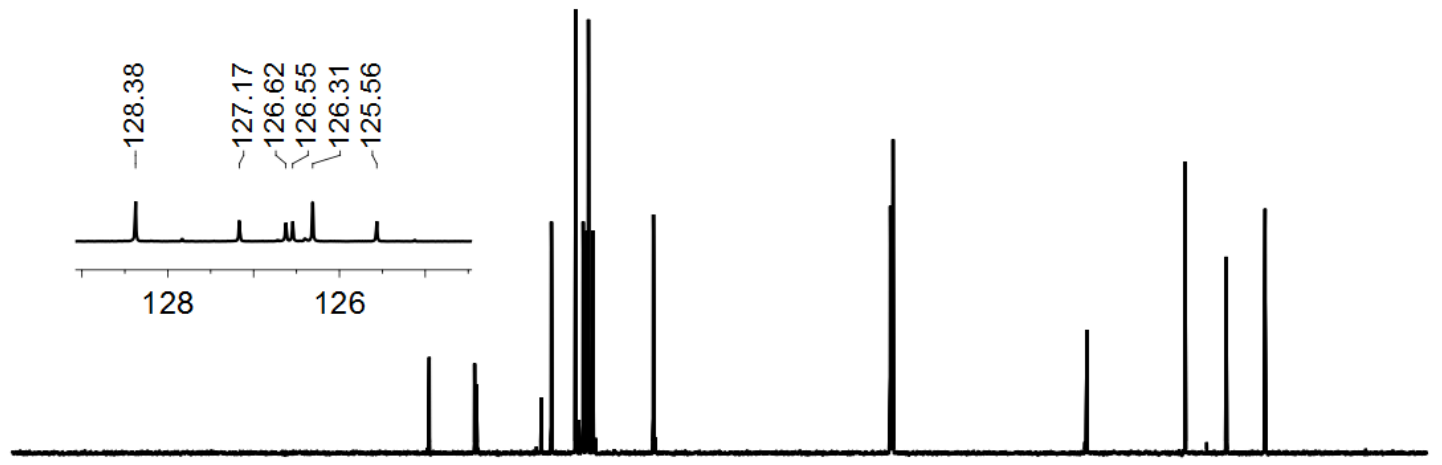

200

170

140

80

40 


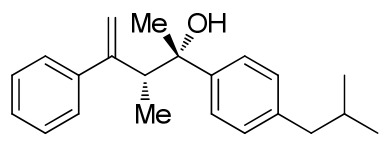

3i

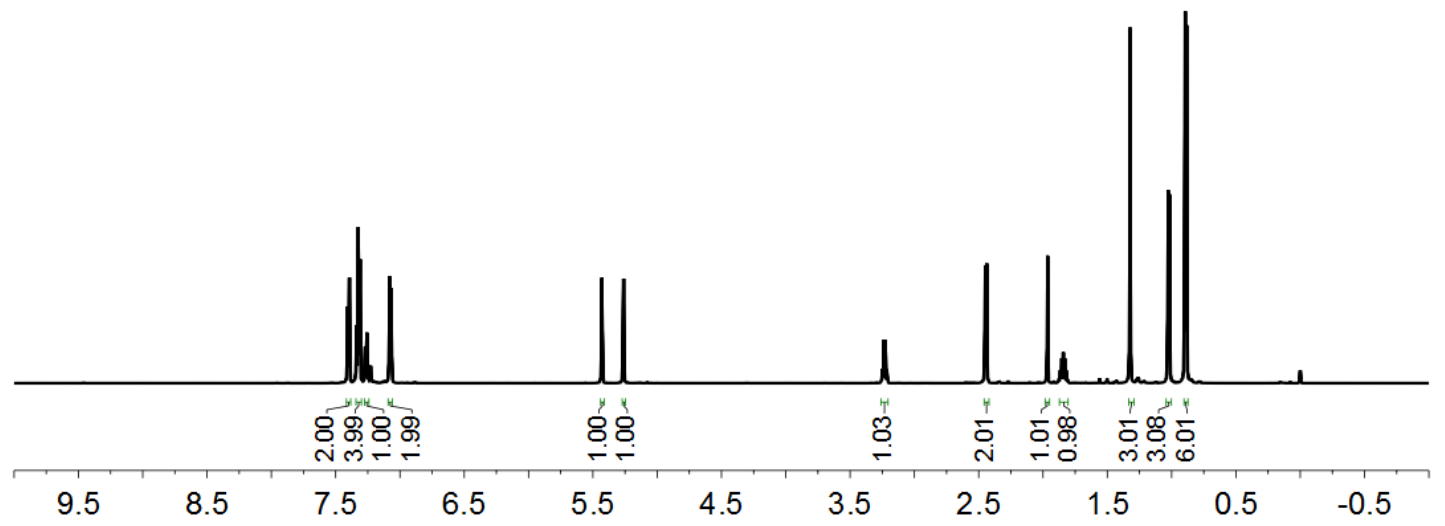

ఝ œ œ

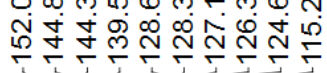

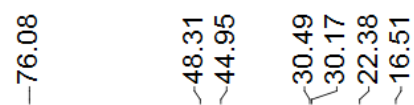

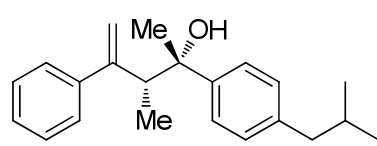

3i

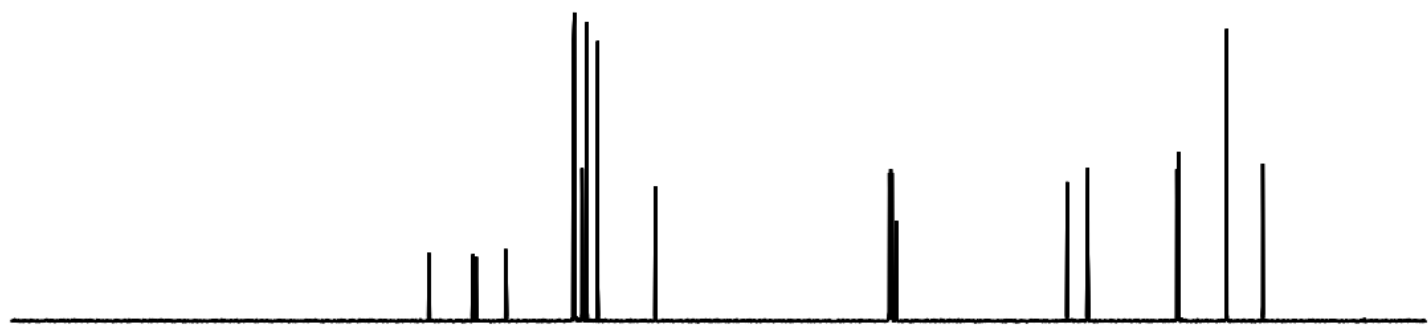


<smiles>C=C(c1ccccc1)[C@H](C)[C@](C)(O)c1ccc(-c2ccccc2)cc1</smiles>

3j
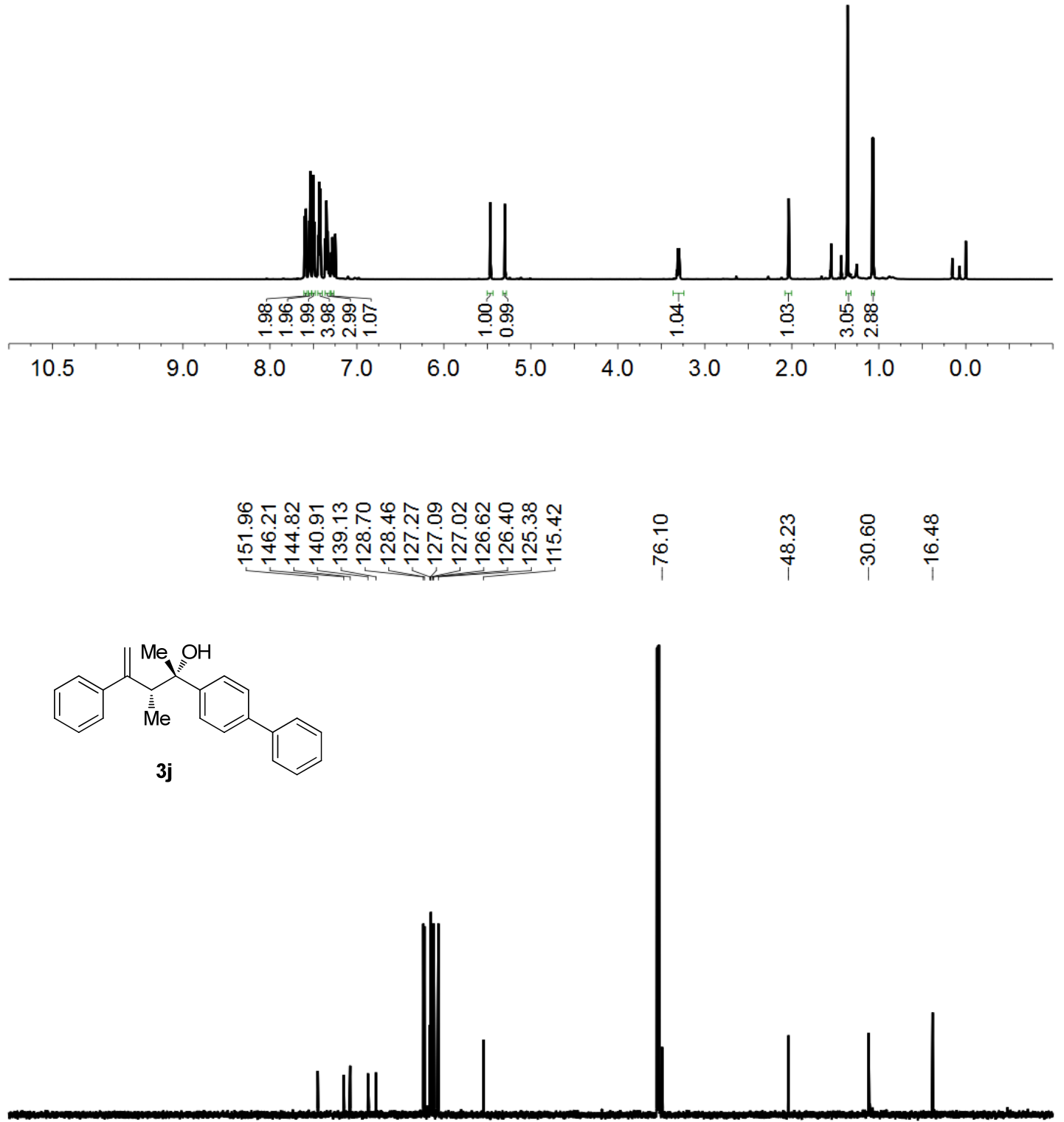


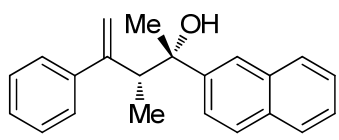

3k

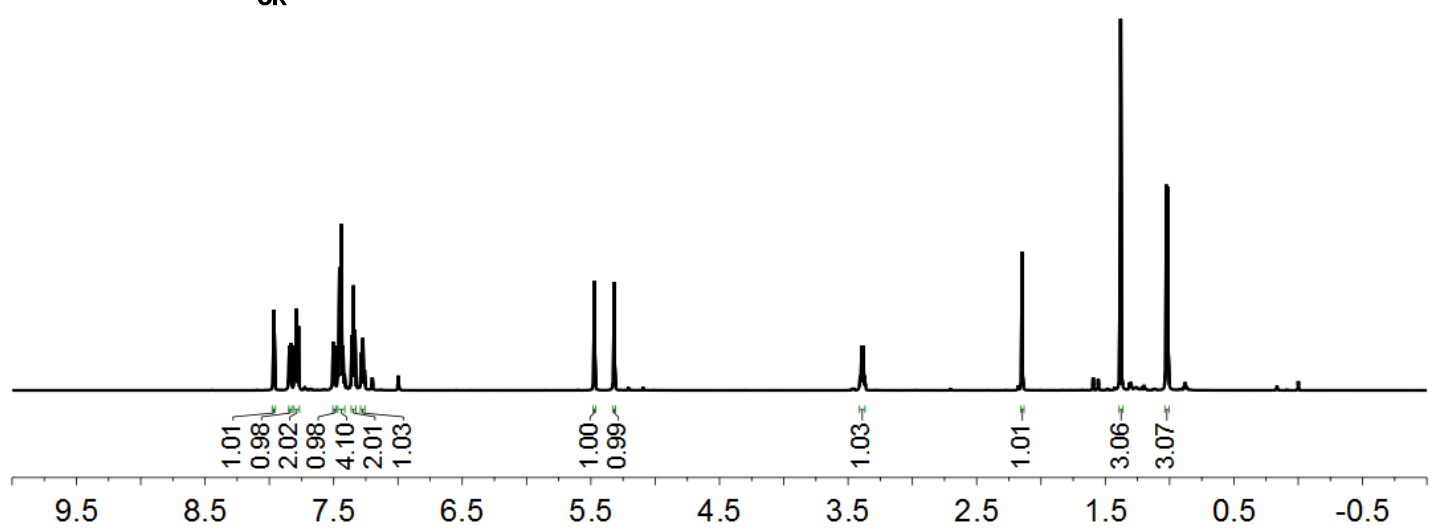

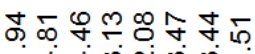

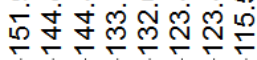

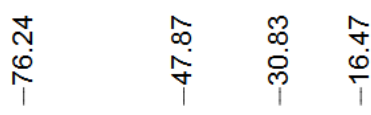

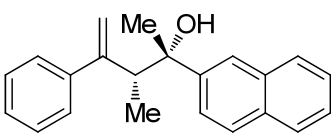

3k

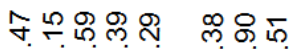

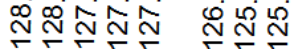

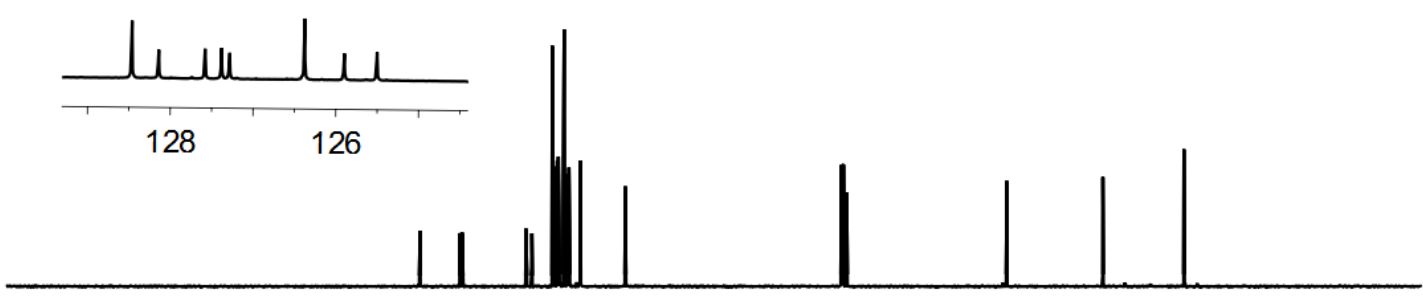

200

170

140

110

80

$60 \quad 40$

$20 \quad 0$ 


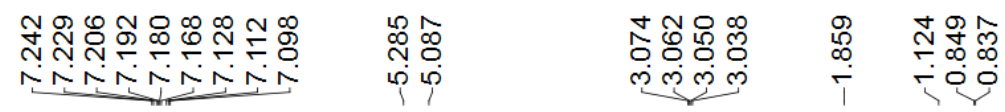
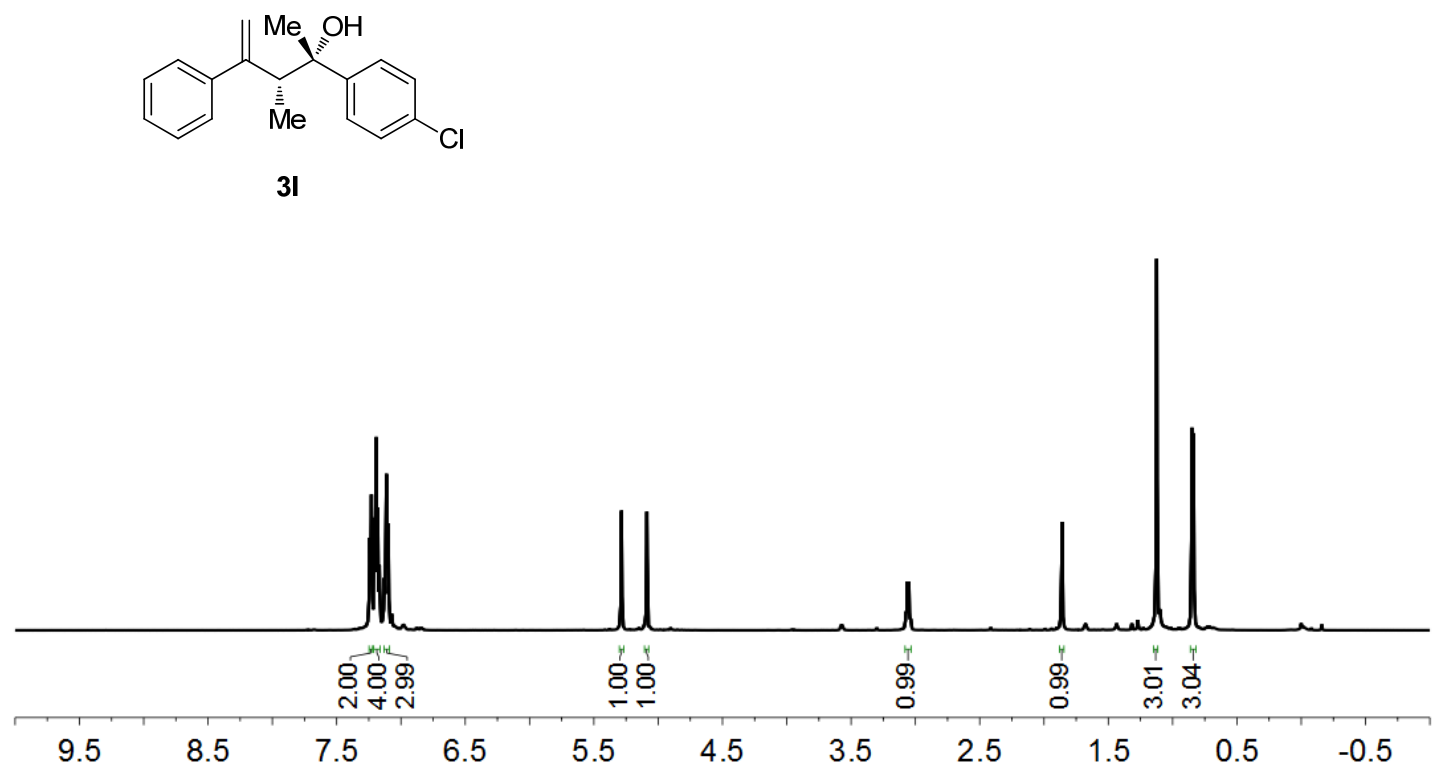

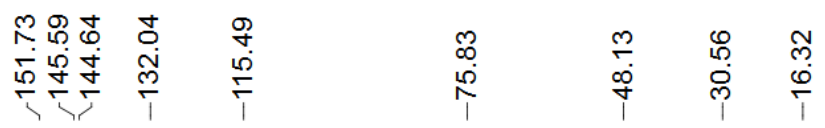

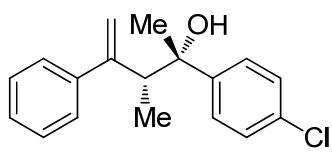

3I

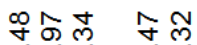

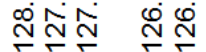

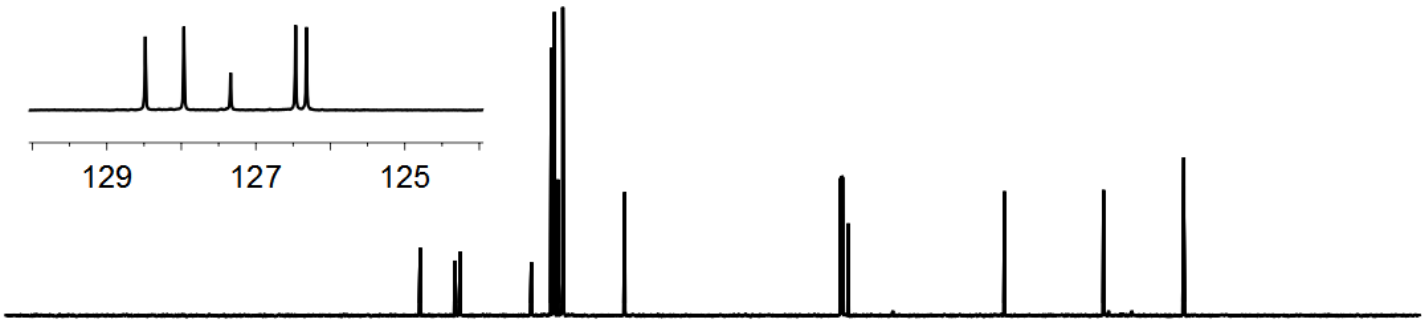

200

170

140

110

80

$\begin{array}{llll}60 & 40 \quad 20 \quad 0\end{array}$ 

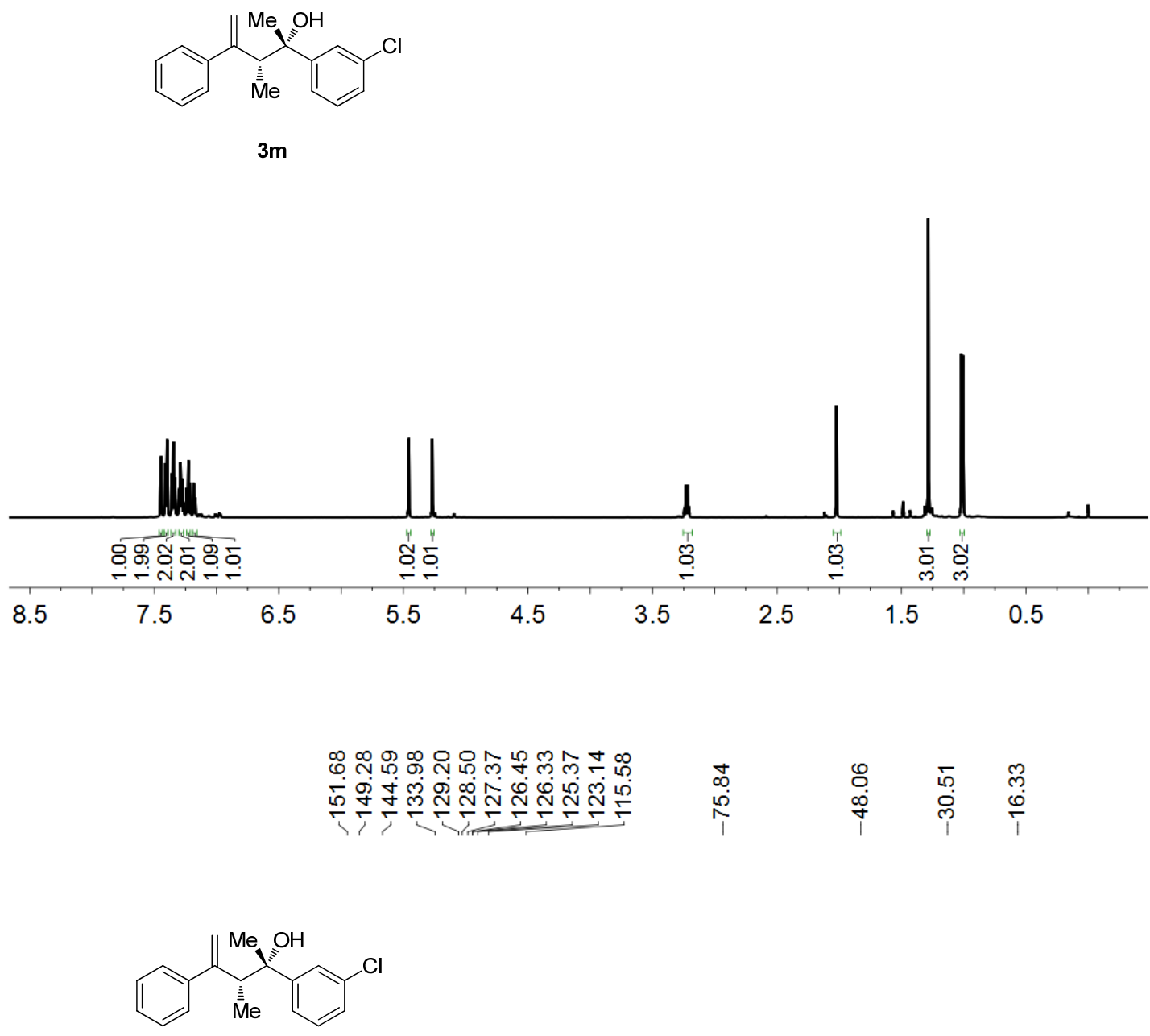

$3 m$

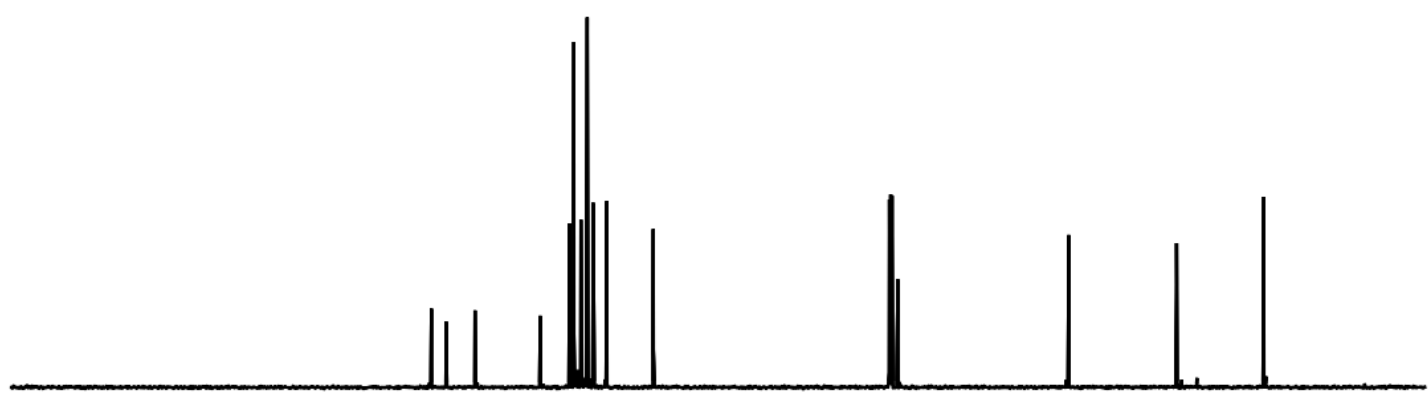




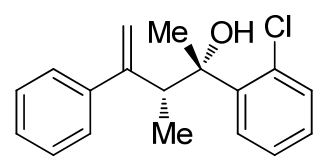

3n

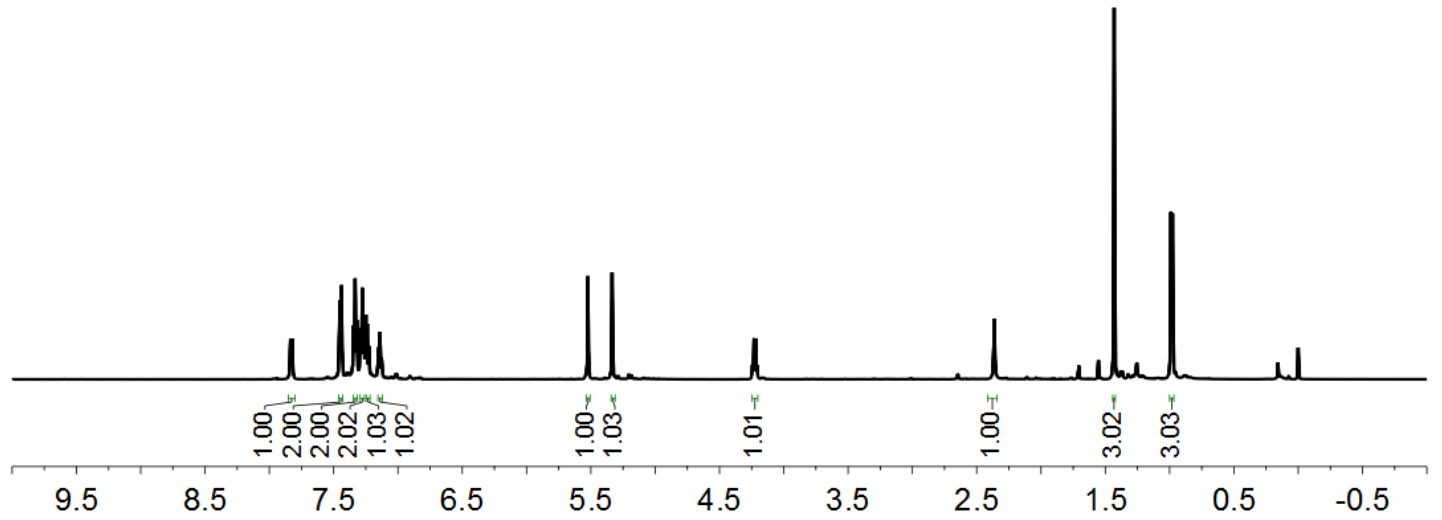<smiles>C=C(c1ccccc1)[C@H](C)[C@@](C)(O)c1ccccc1Cl</smiles>

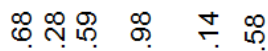

它守寺

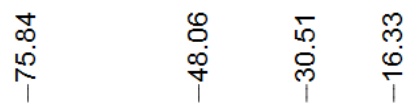

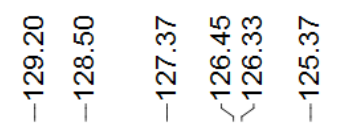

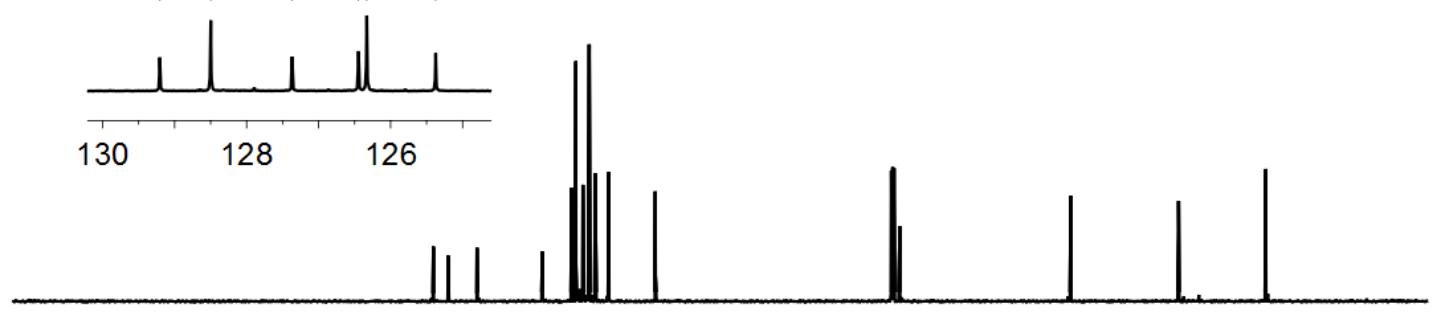

200

170

140

110

80

60

$40 \quad 20 \quad 0$ 

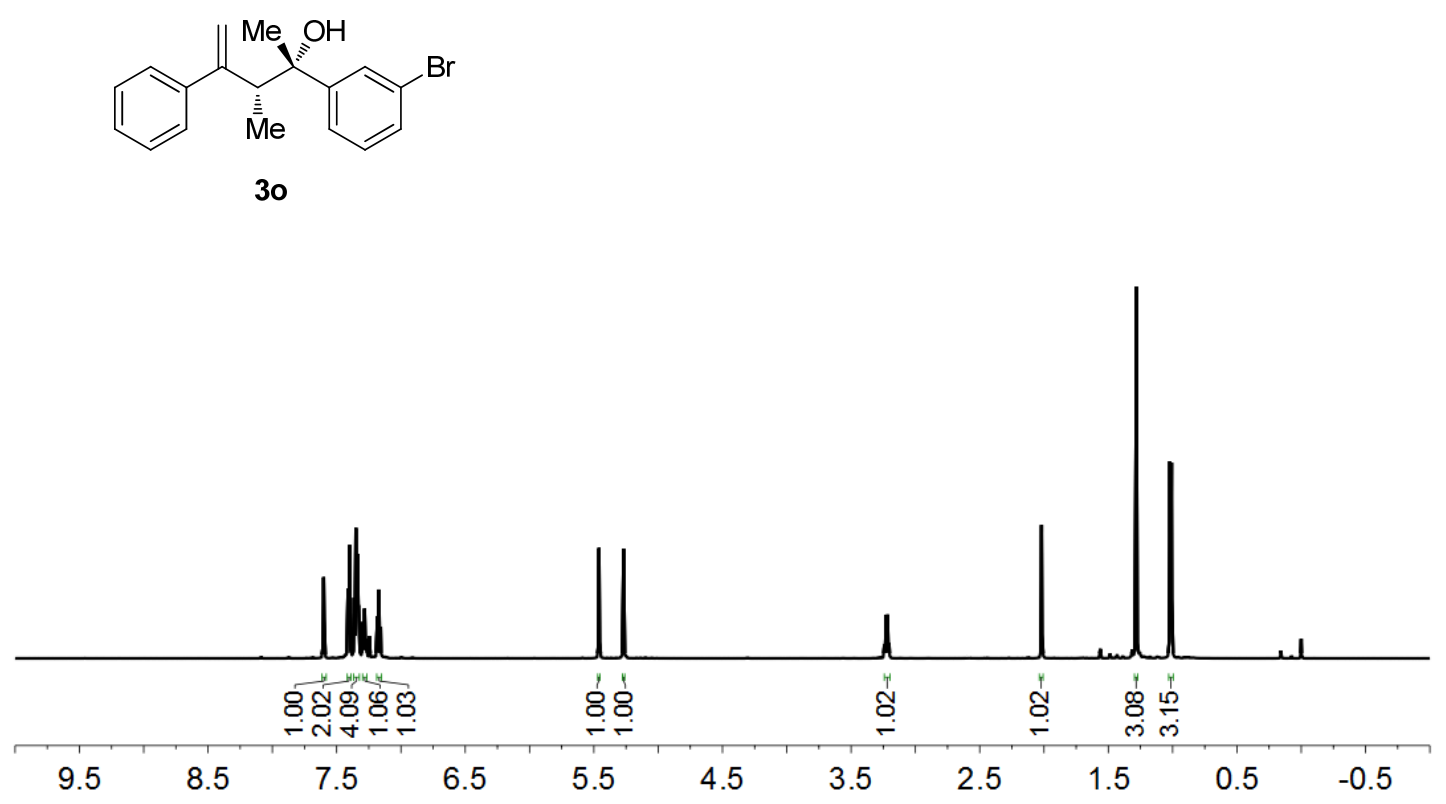

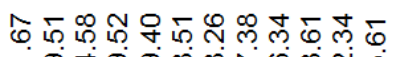

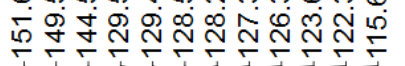
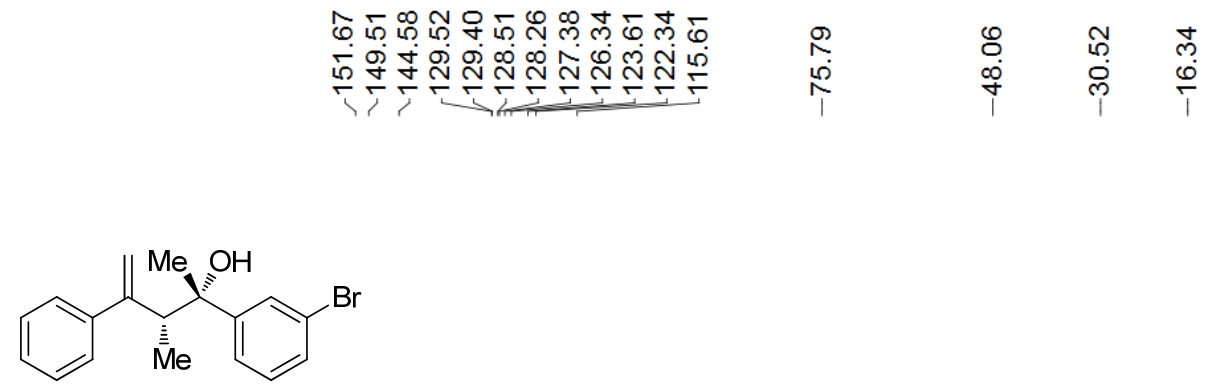

30

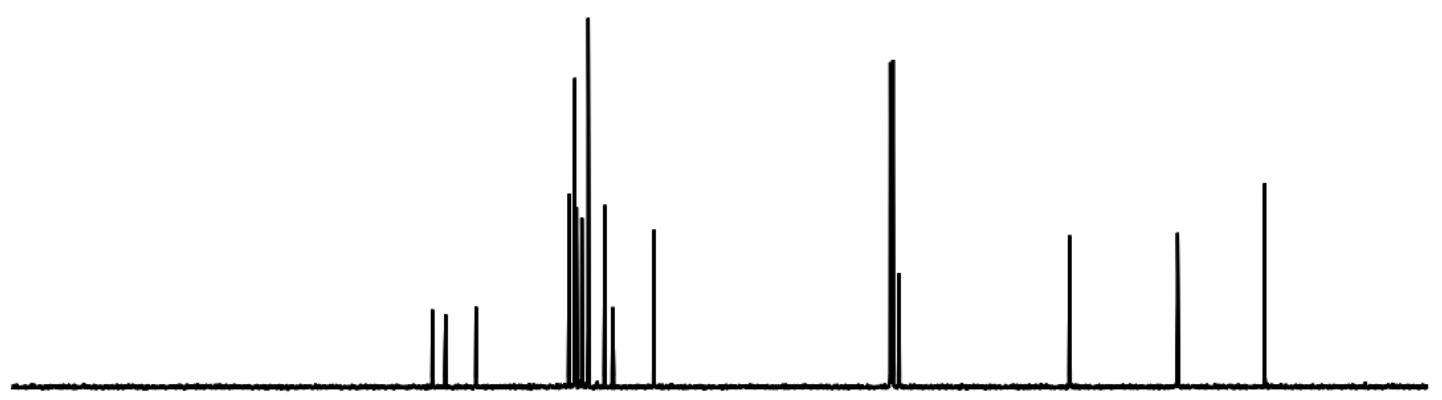

200

170

140

110

80

40

$20 \quad 0$ 

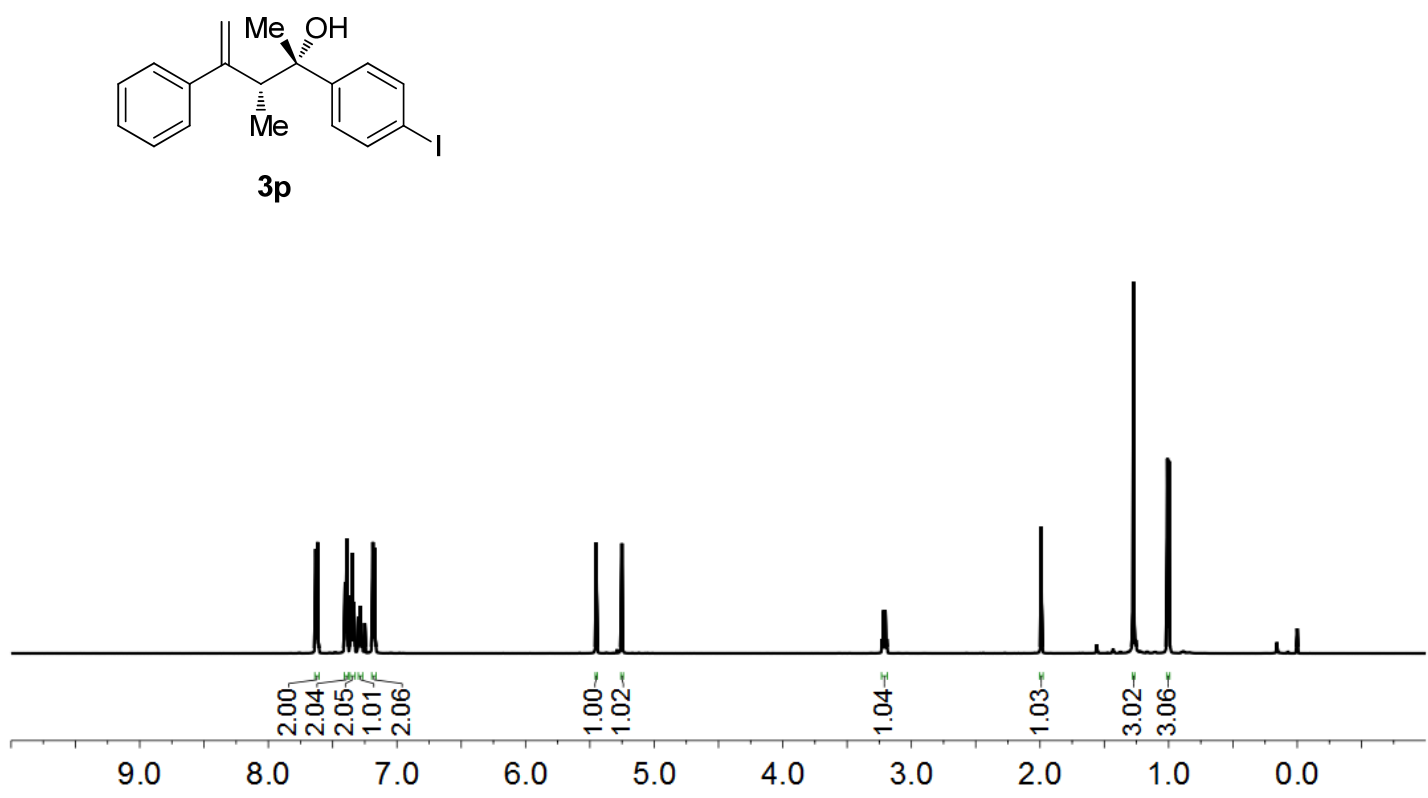

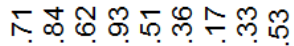

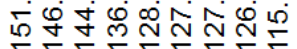

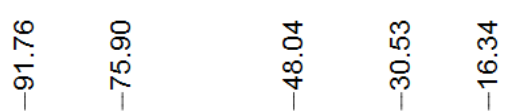<smiles>[Y9]C(C(=C)c1ccccc1)C(O)(O)c1ccc(I)cc1</smiles>

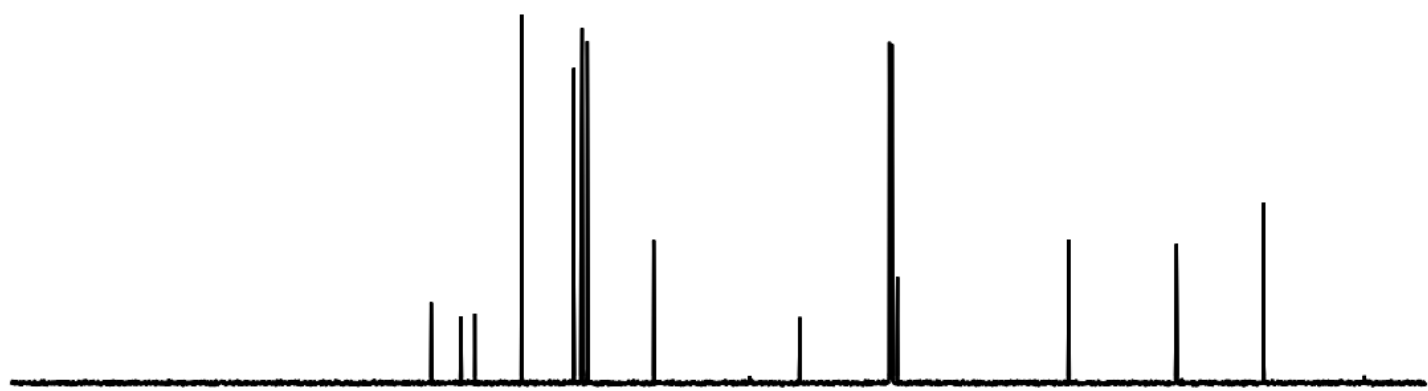




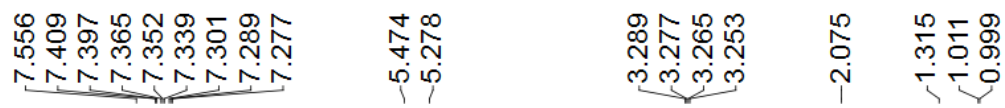<smiles>C=C(c1ccccc1)[C@H](C)[C@](C)(O)c1ccc(C(F)(F)F)cc1</smiles>

$3 q$
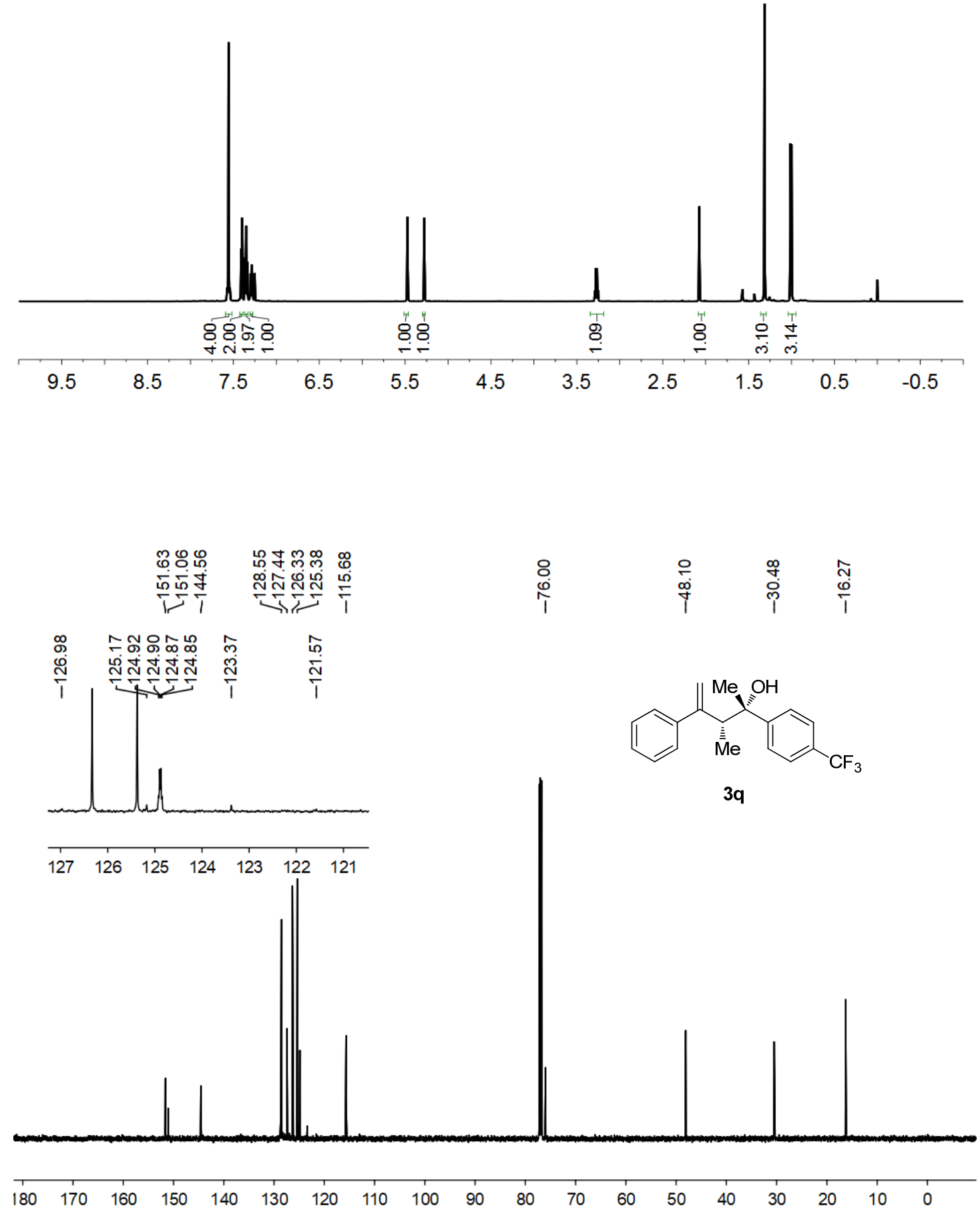


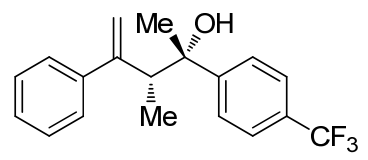

$3 q$

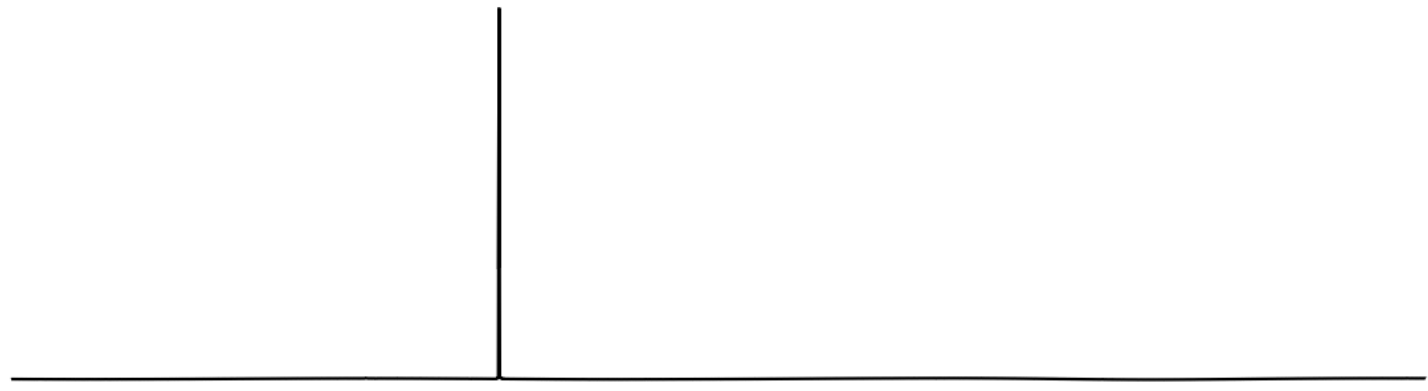



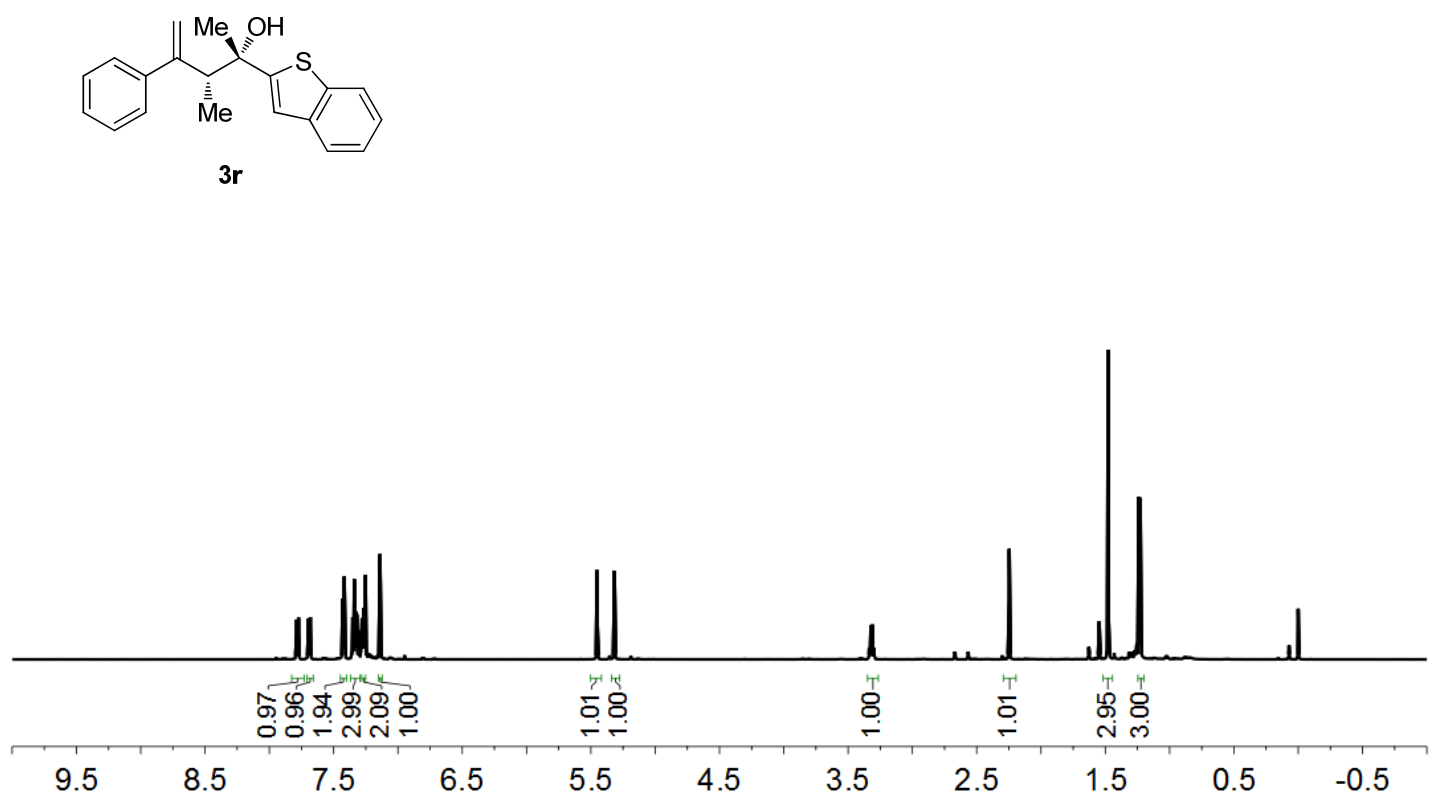

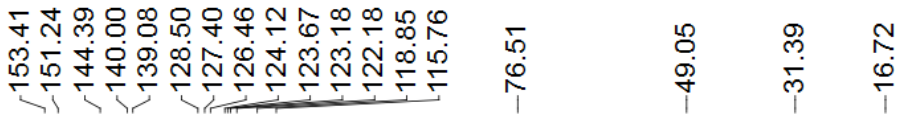

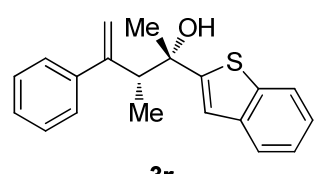

$3 r$

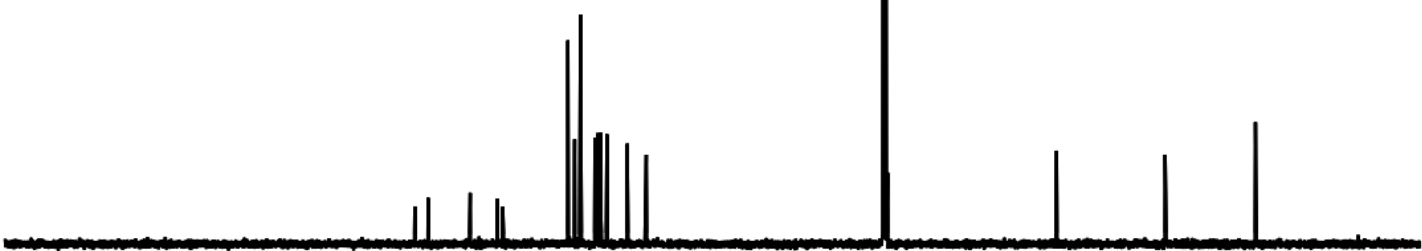

200

170

140

110

80

60

40

20 


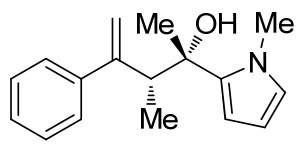

$3 s$

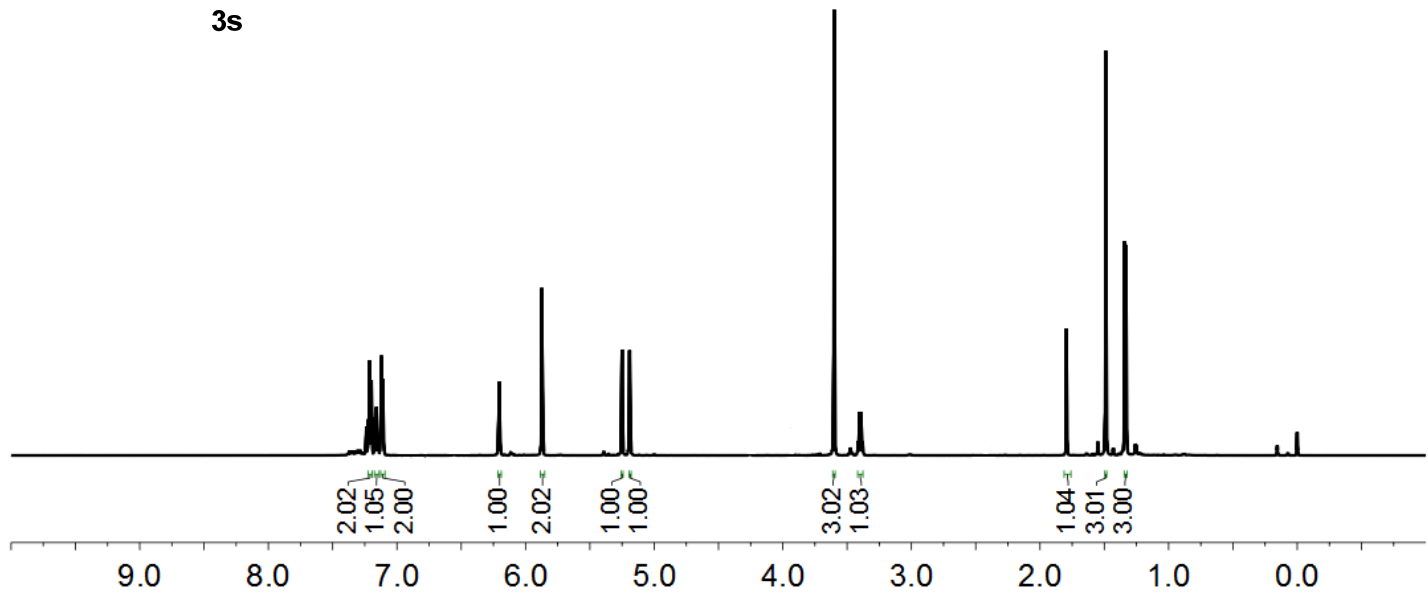

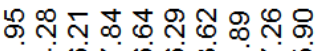

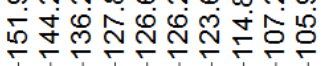
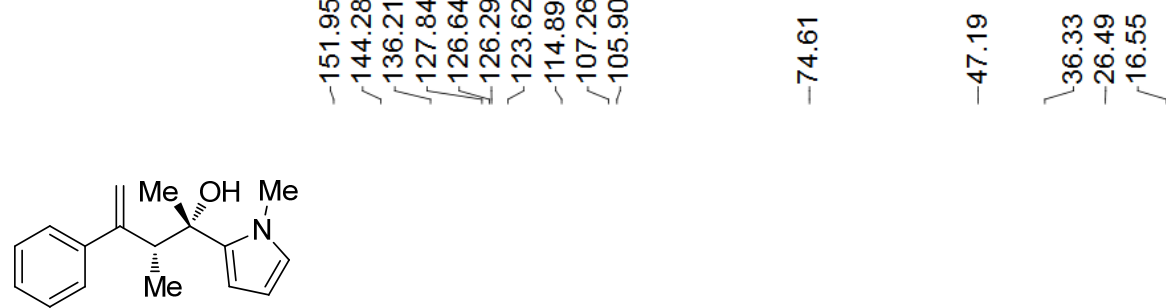

3s

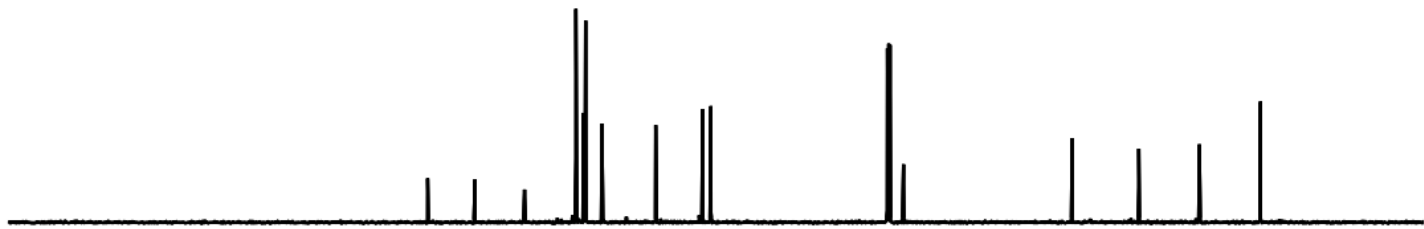




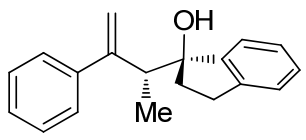

$3 t$

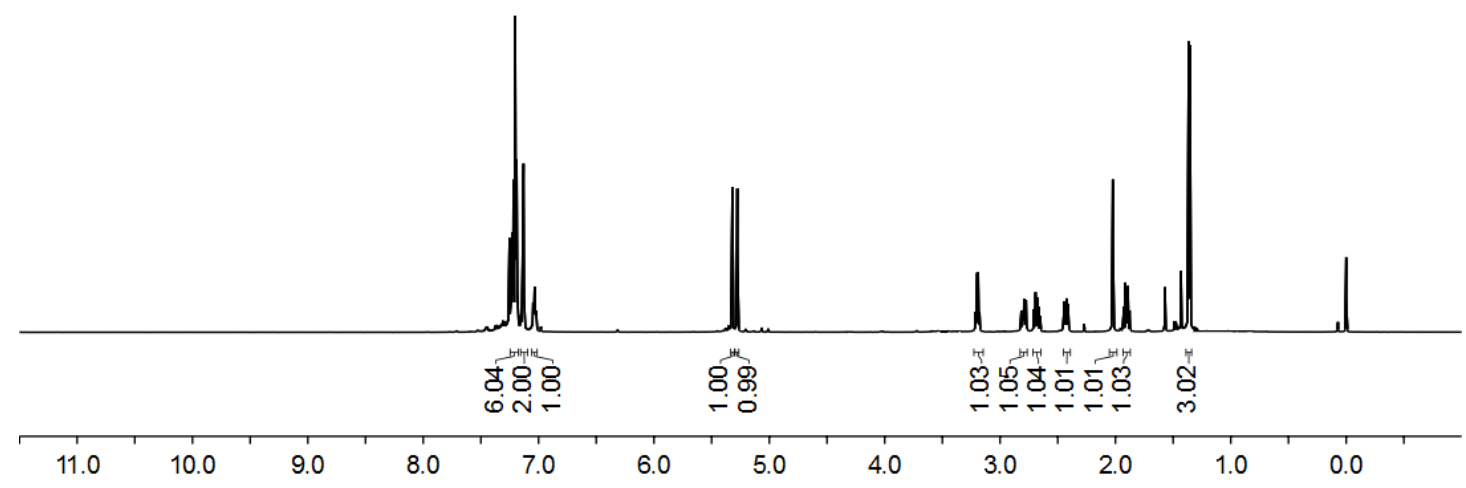

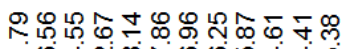

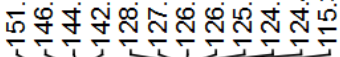

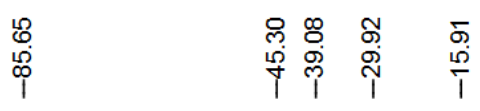

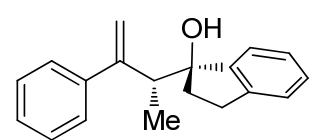

$3 t$

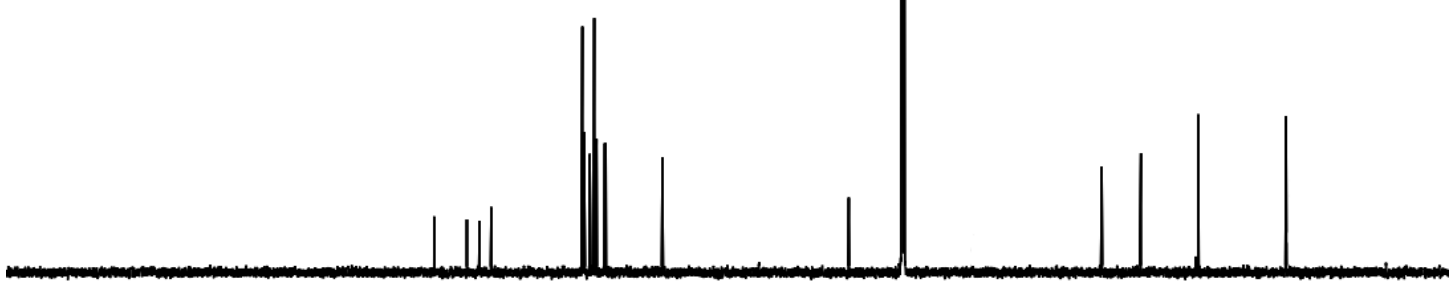

190

170

150

130

110

$\begin{array}{lllllllllll}90 & 80 & 70 & 60 & 50 & 40 & 30 & 20 & 10 & 0 & -1\end{array}$ 


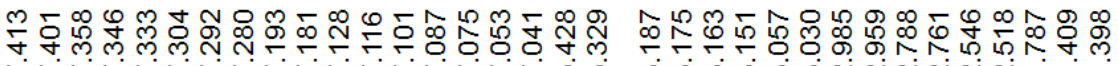

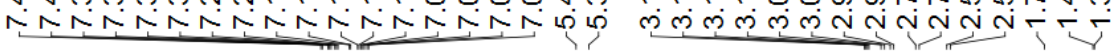
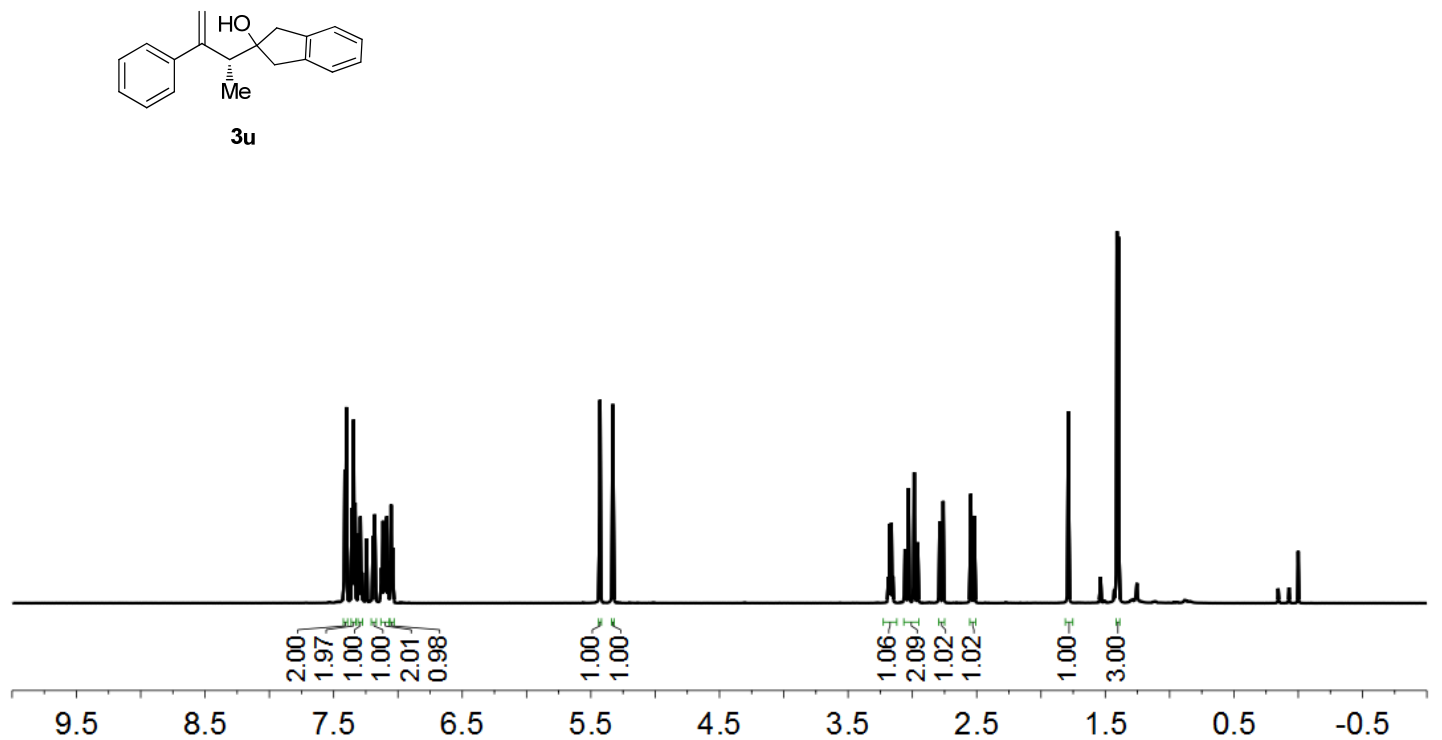

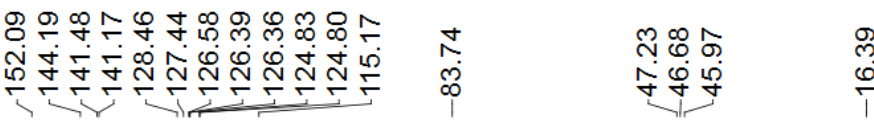
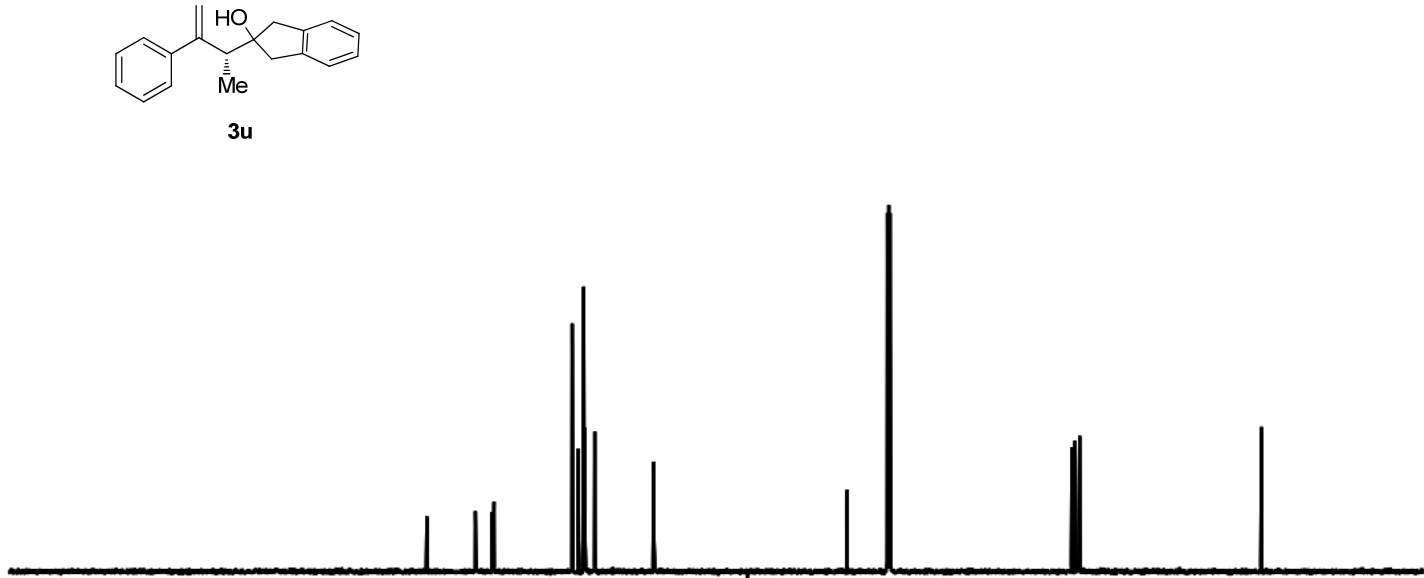

200

170

140

110

$80 \quad 60$

60

40

20

0 


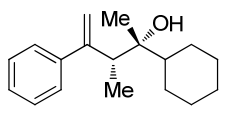

$3 \mathbf{v}$
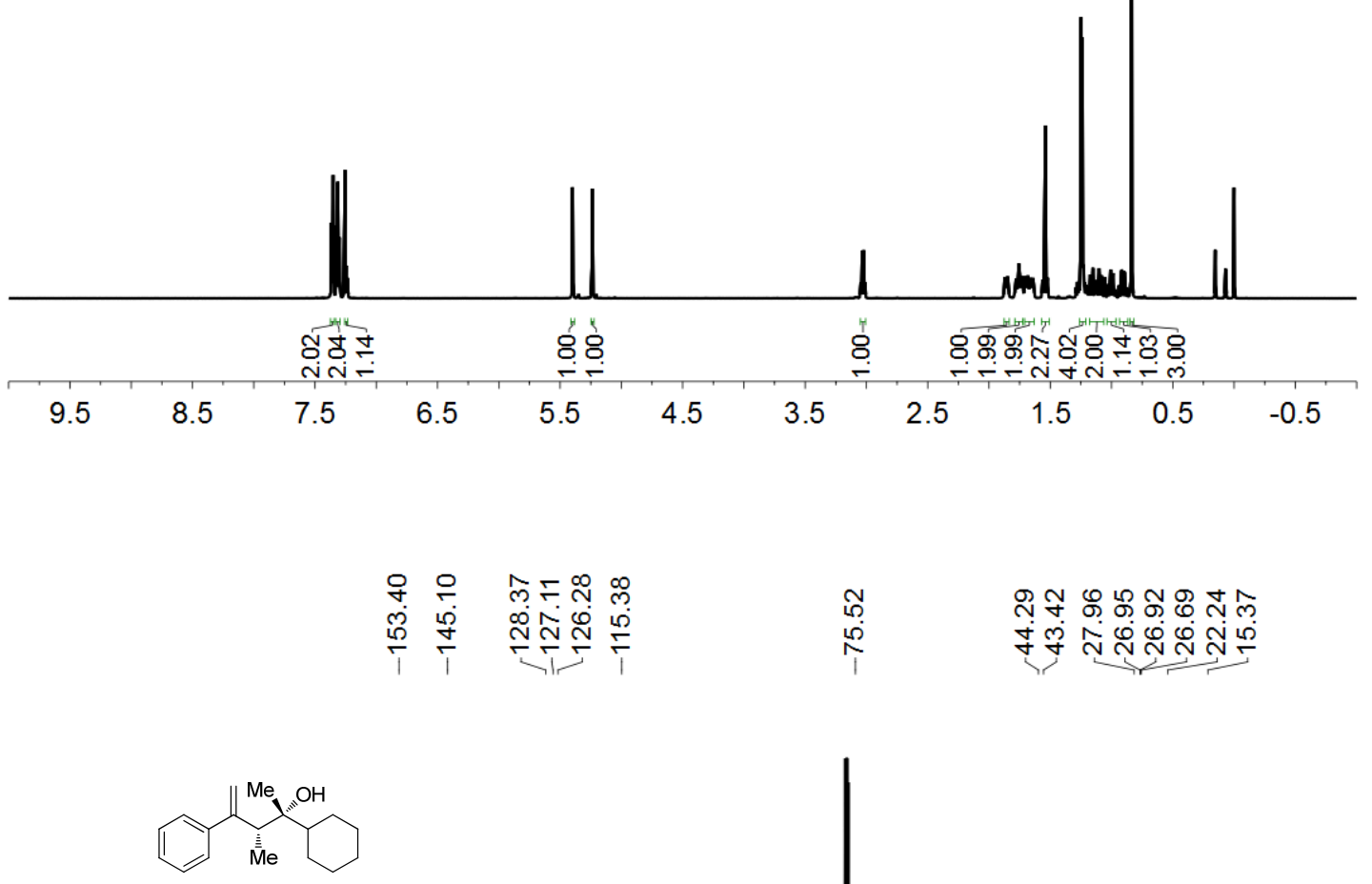

$3 \mathbf{v}$

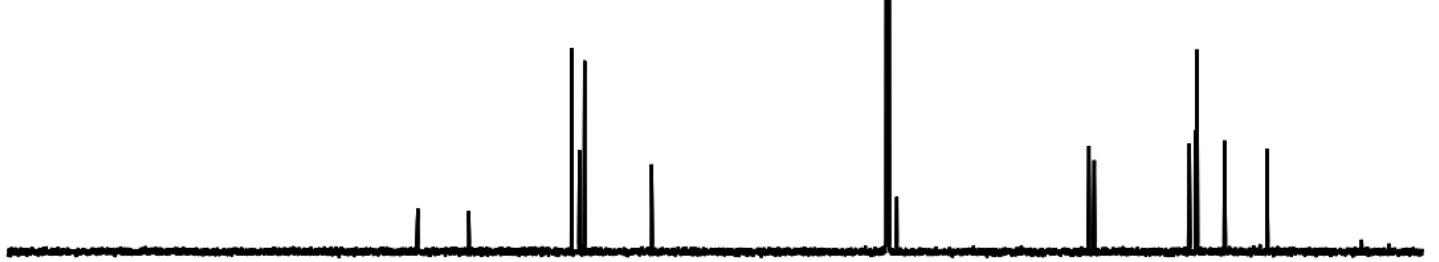

200

170

140

110

$80 \quad 60$

60

40

20 


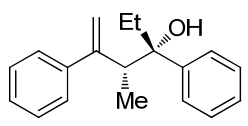

$3 w$
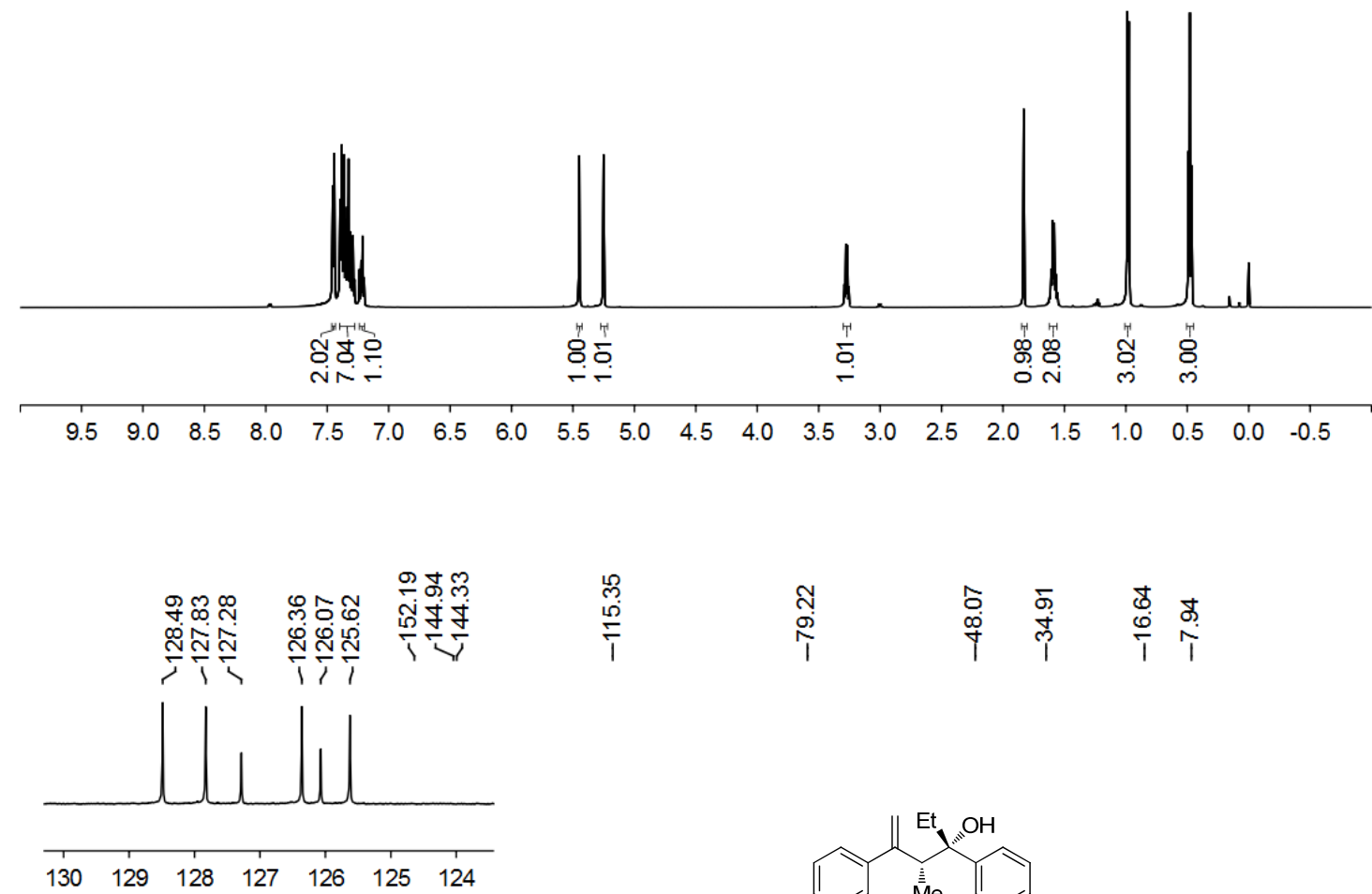

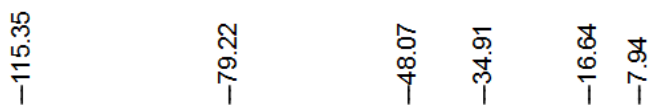

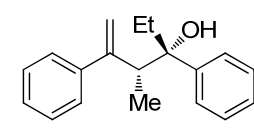

$3 w$

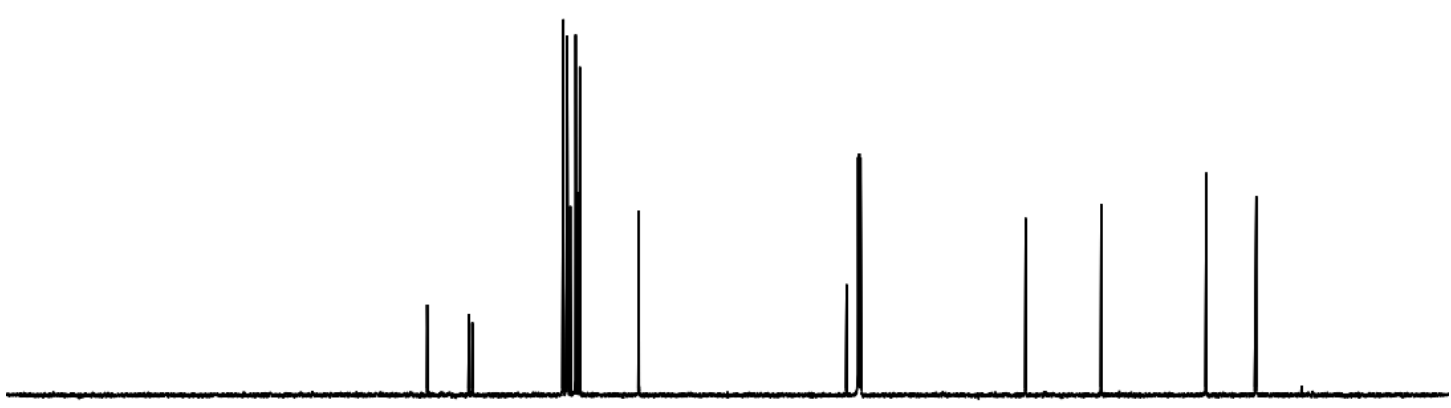




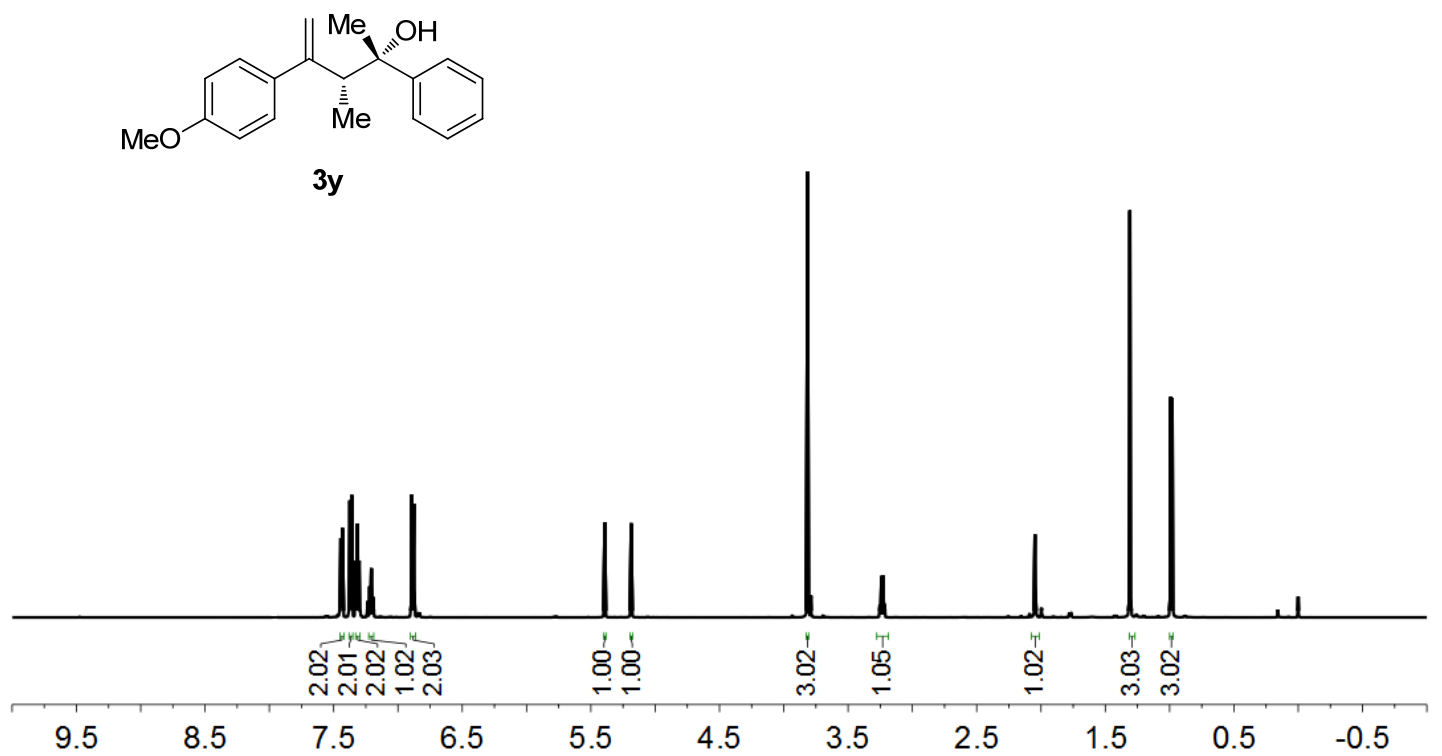

कิ

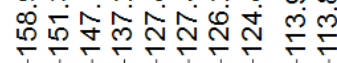

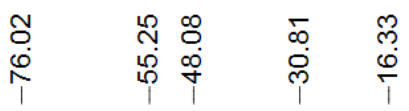
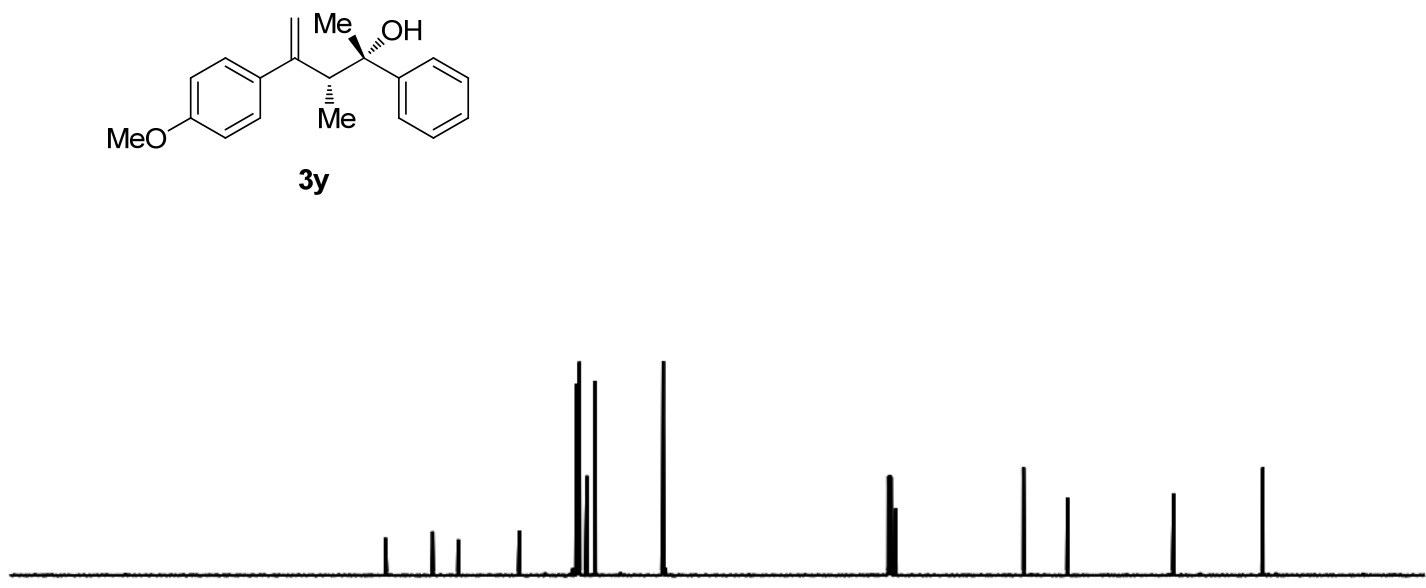

200

170

140

60

$40 \quad 20$ 


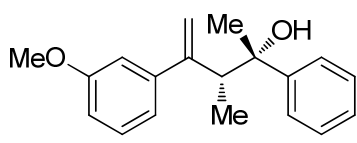

$3 z$

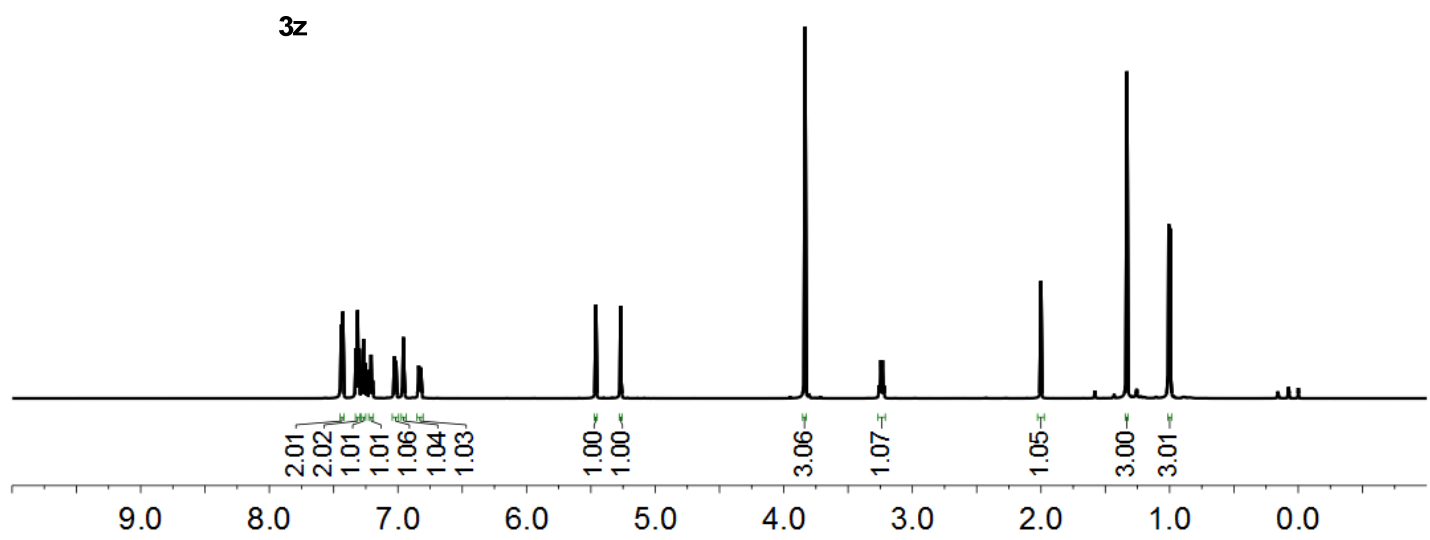

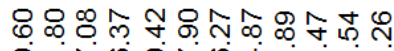

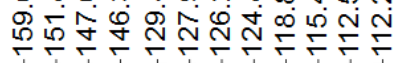

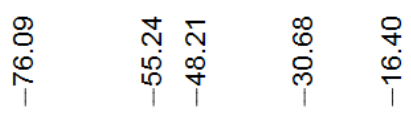

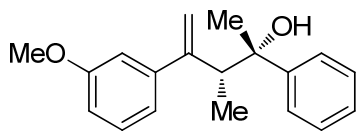

$3 z$

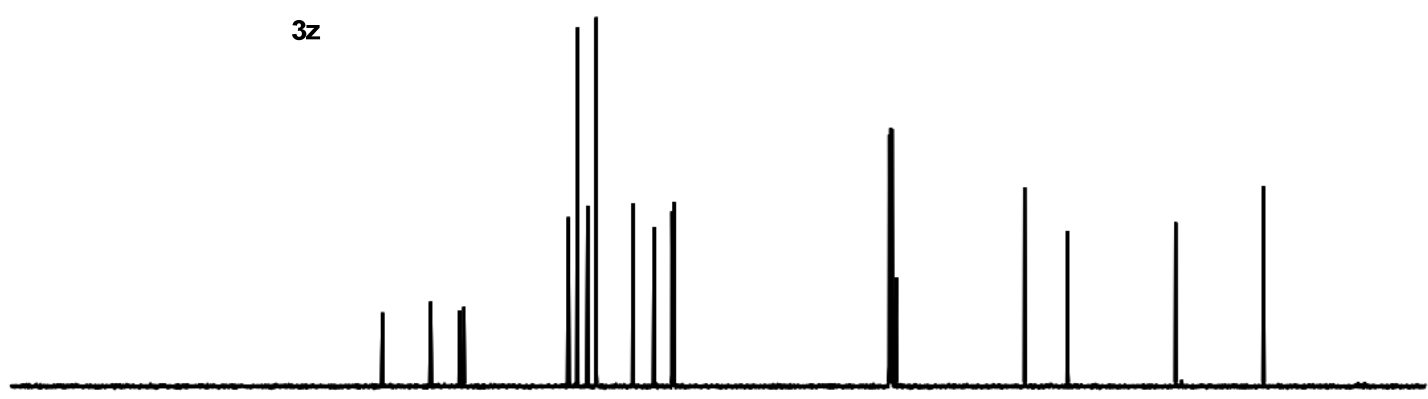

200

170

140

110

60

$20 \quad 0$ 


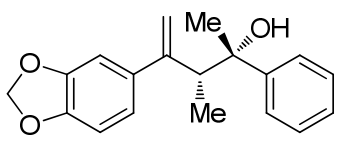

3aa

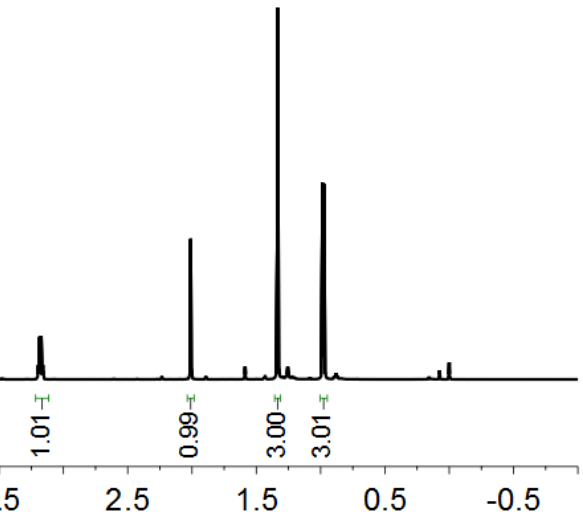

공ำ

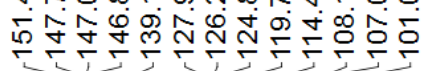

0
0
1

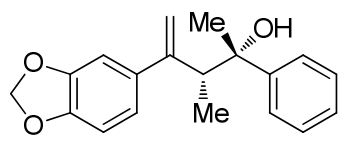

3aa

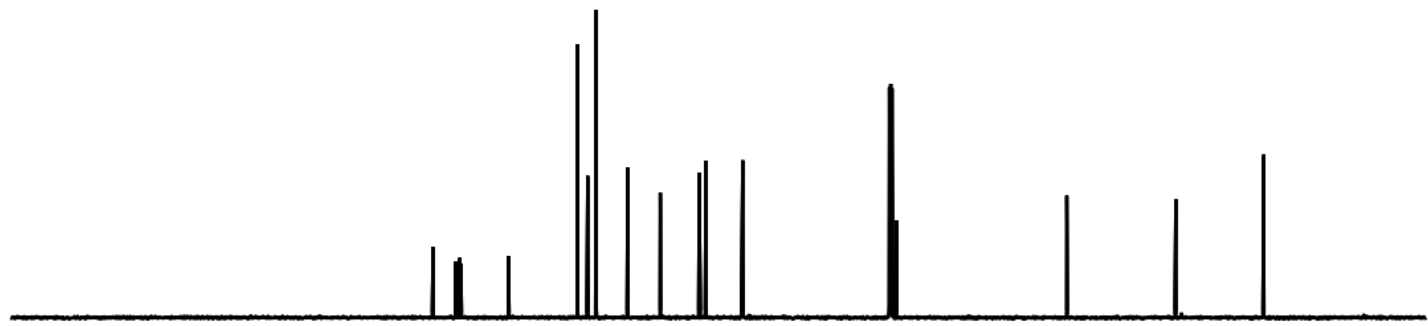




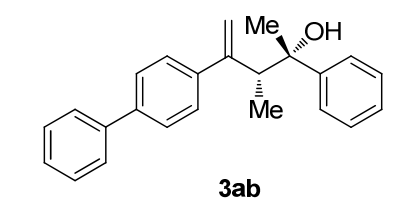

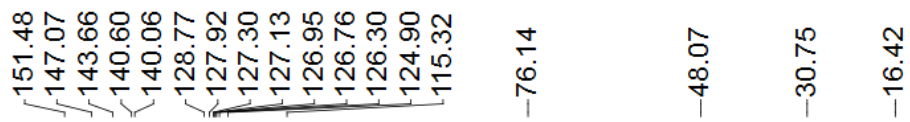
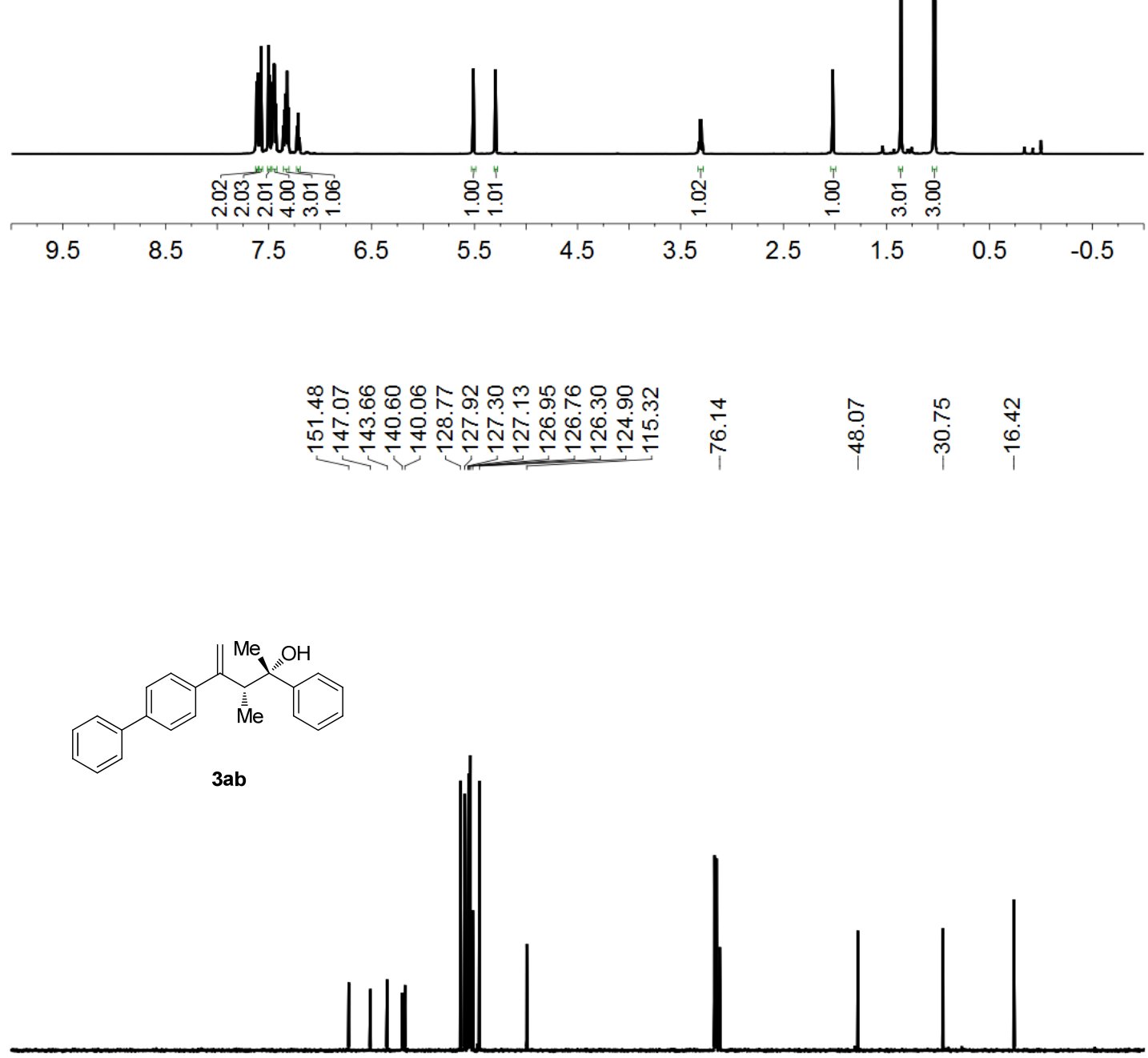

200

170

140

110

80

60

0 


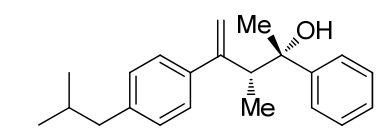

$3 a c$

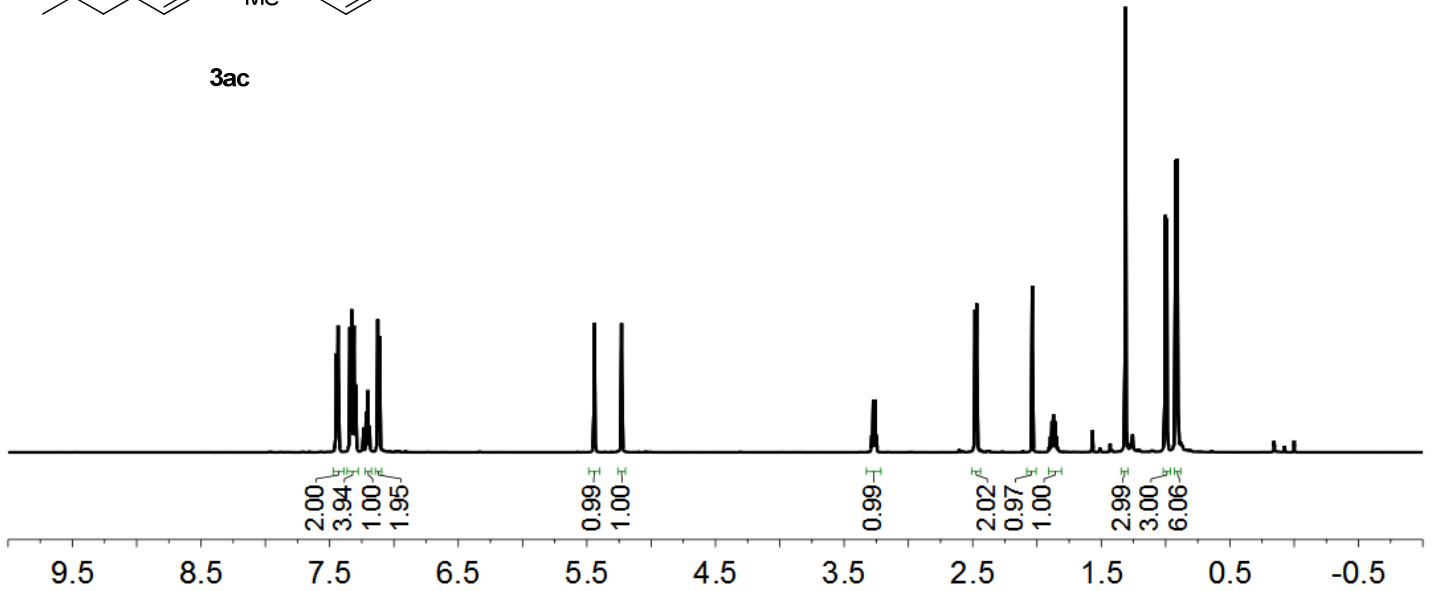

๓

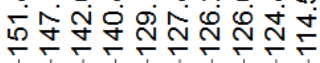

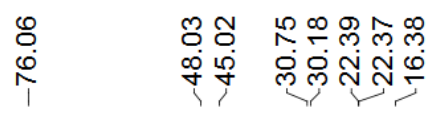

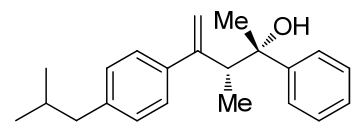

$3 a c$

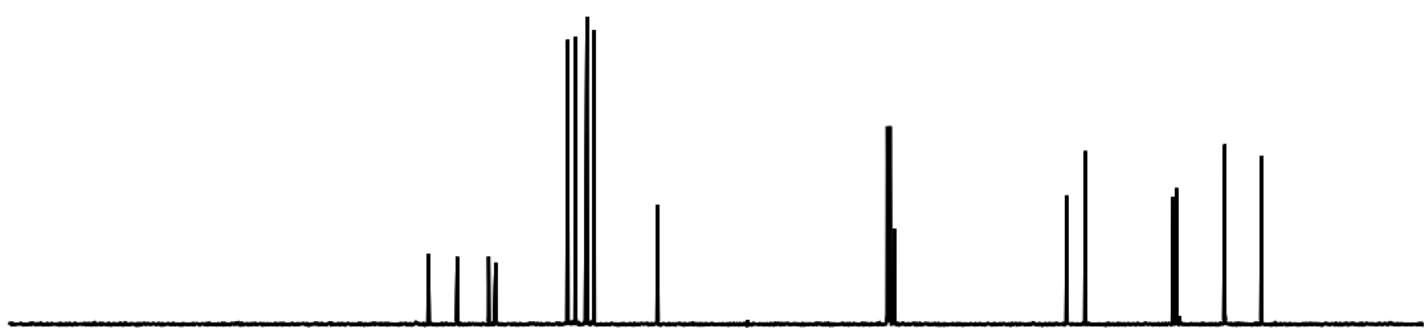

200

170

140

110

80

60

40

20

0 


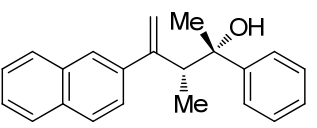

3ad

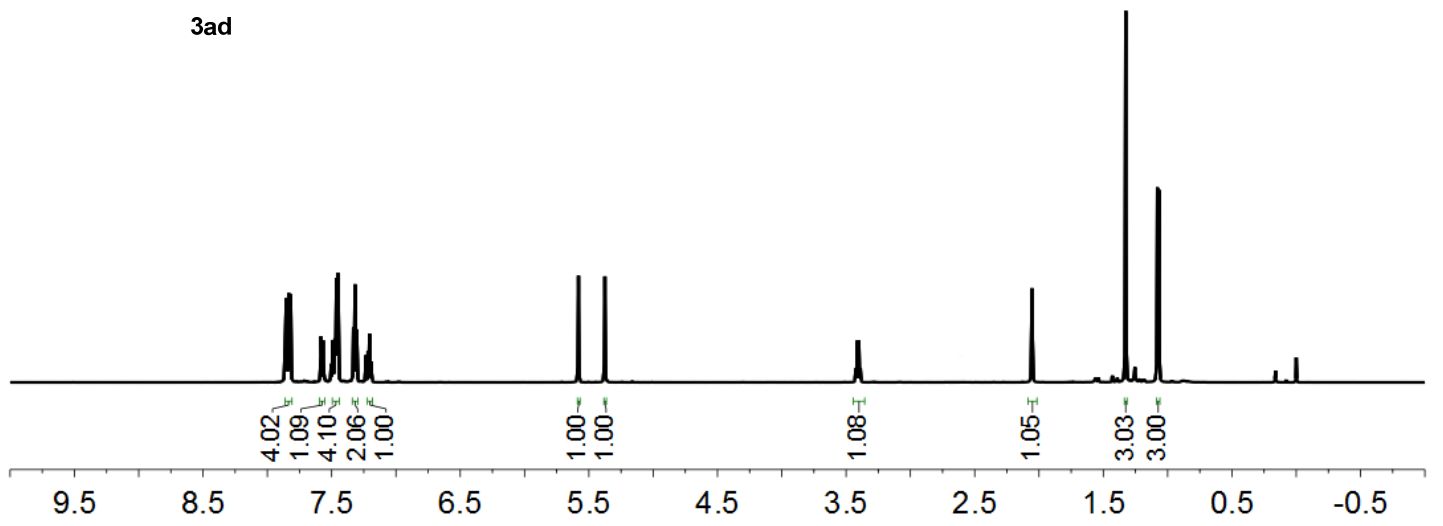

б

它寺讪

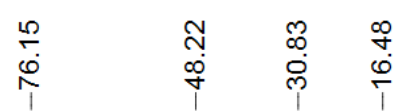
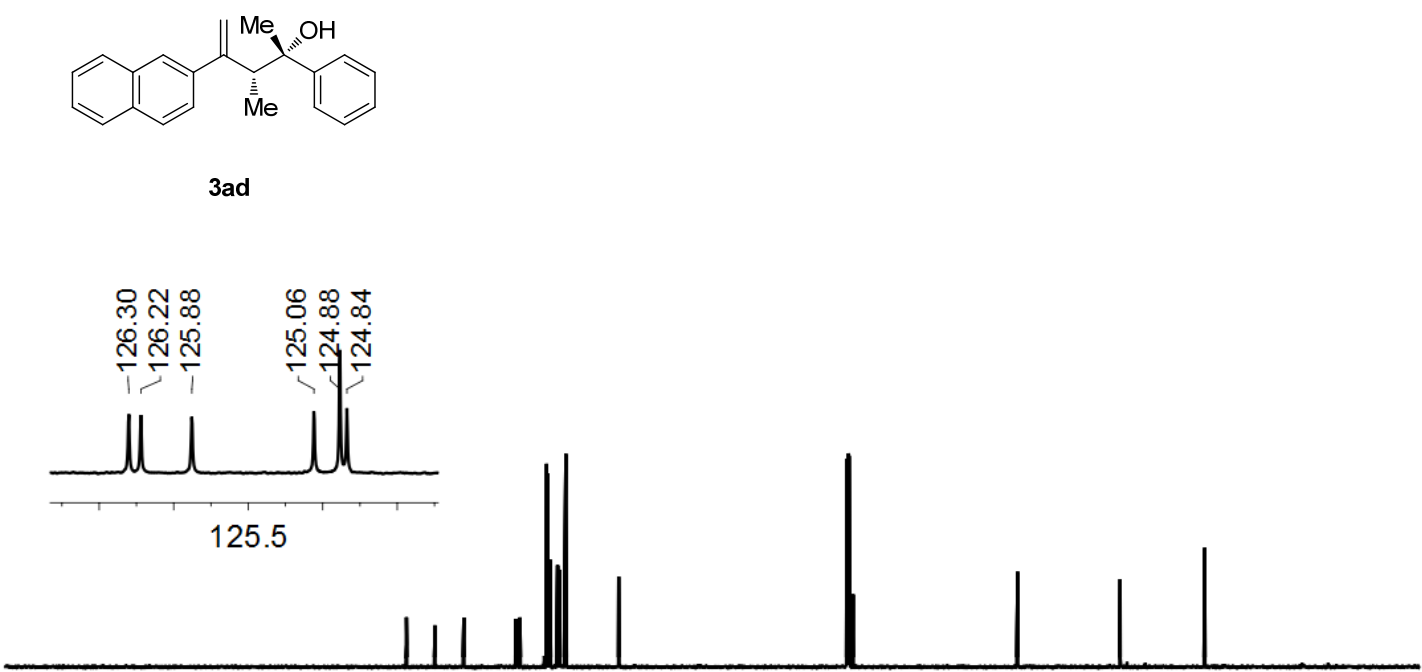

200

170

140

110

$80 \quad 60$

$40 \quad 20 \quad 0 \quad-10$ 


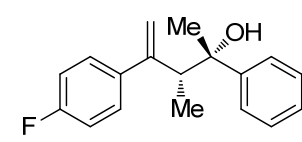

3ae

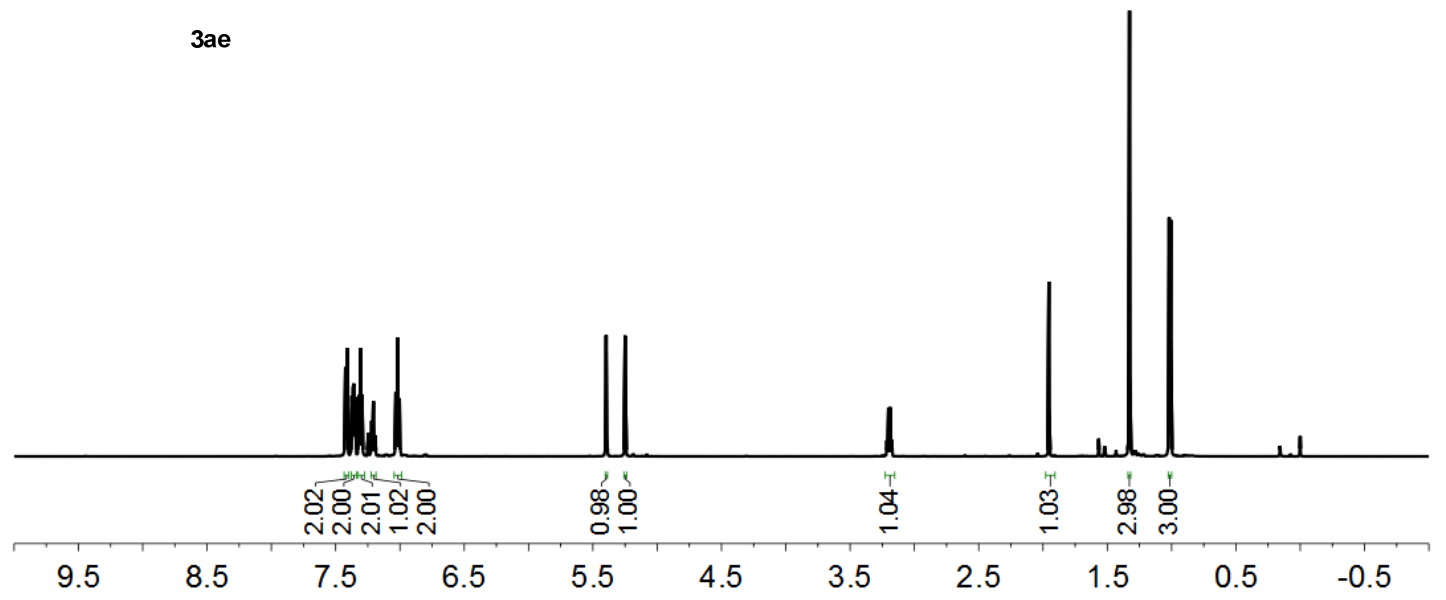

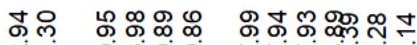

ชั่

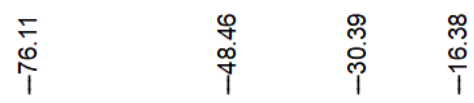

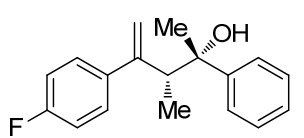

3ae

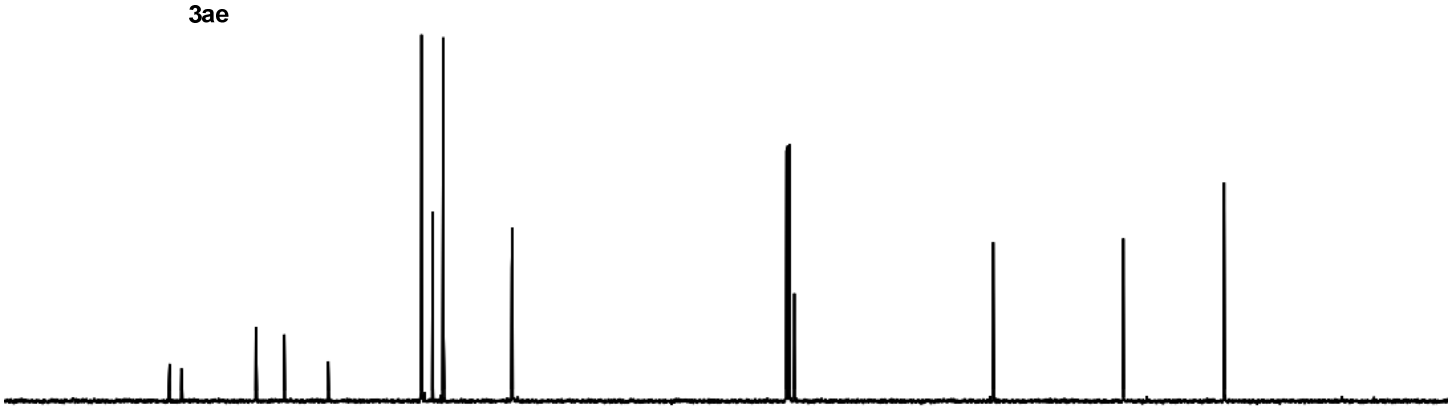

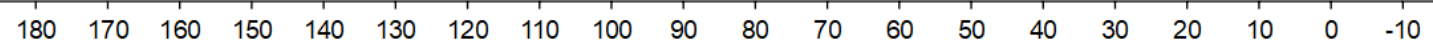




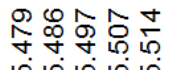

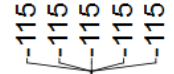

我

$3 a e$

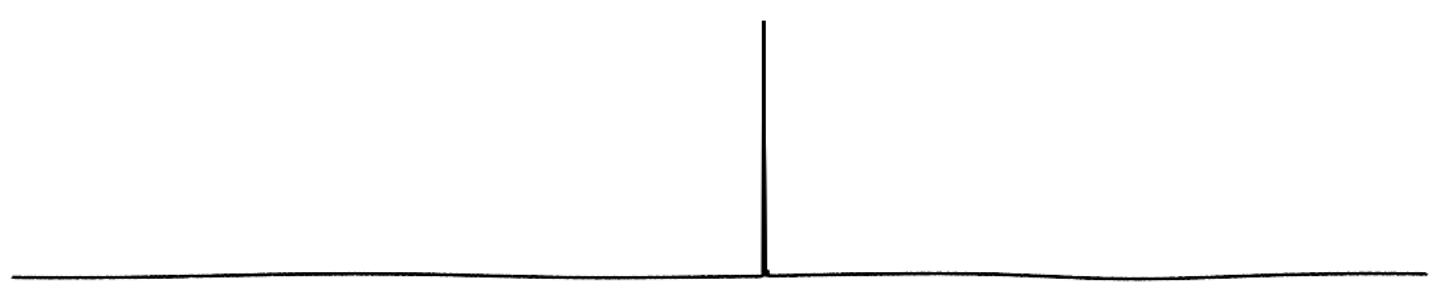




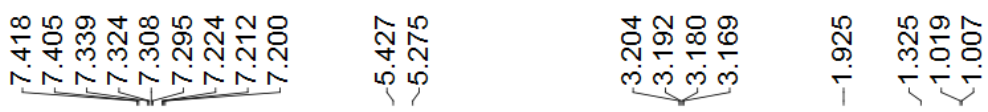
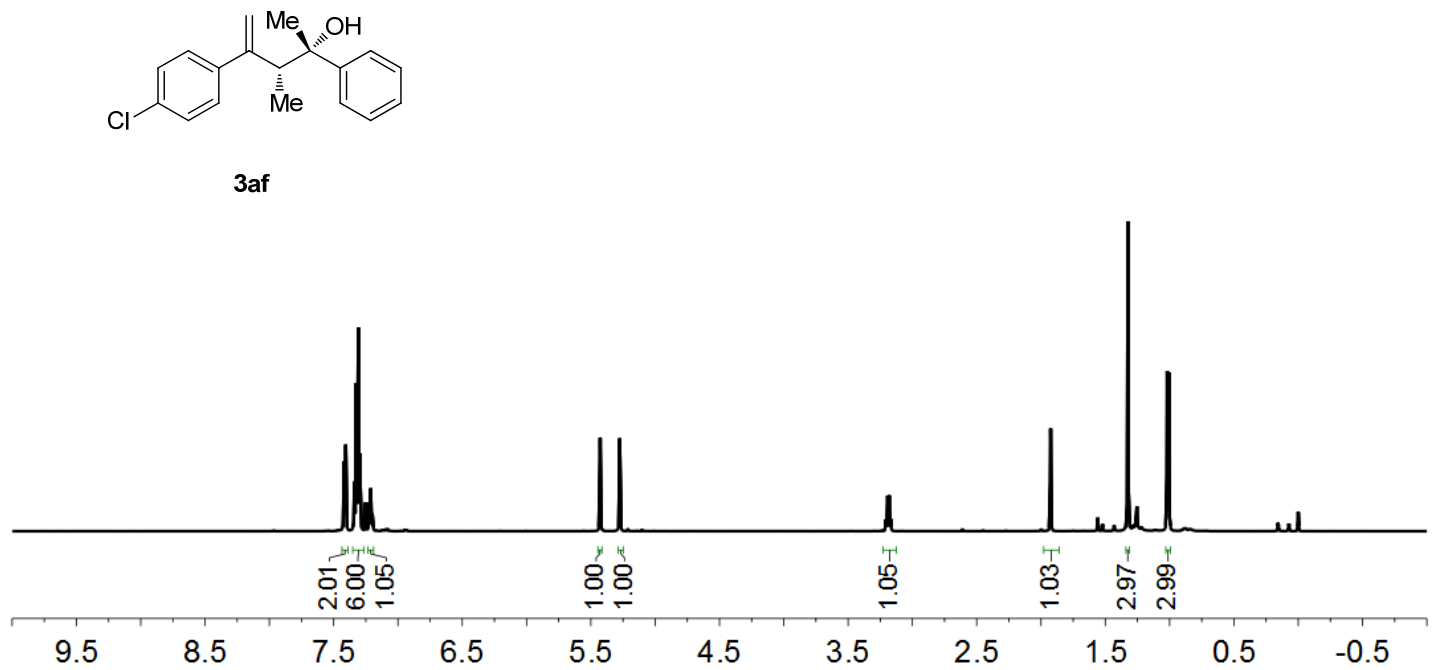

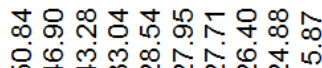

官

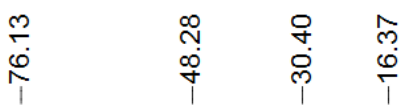

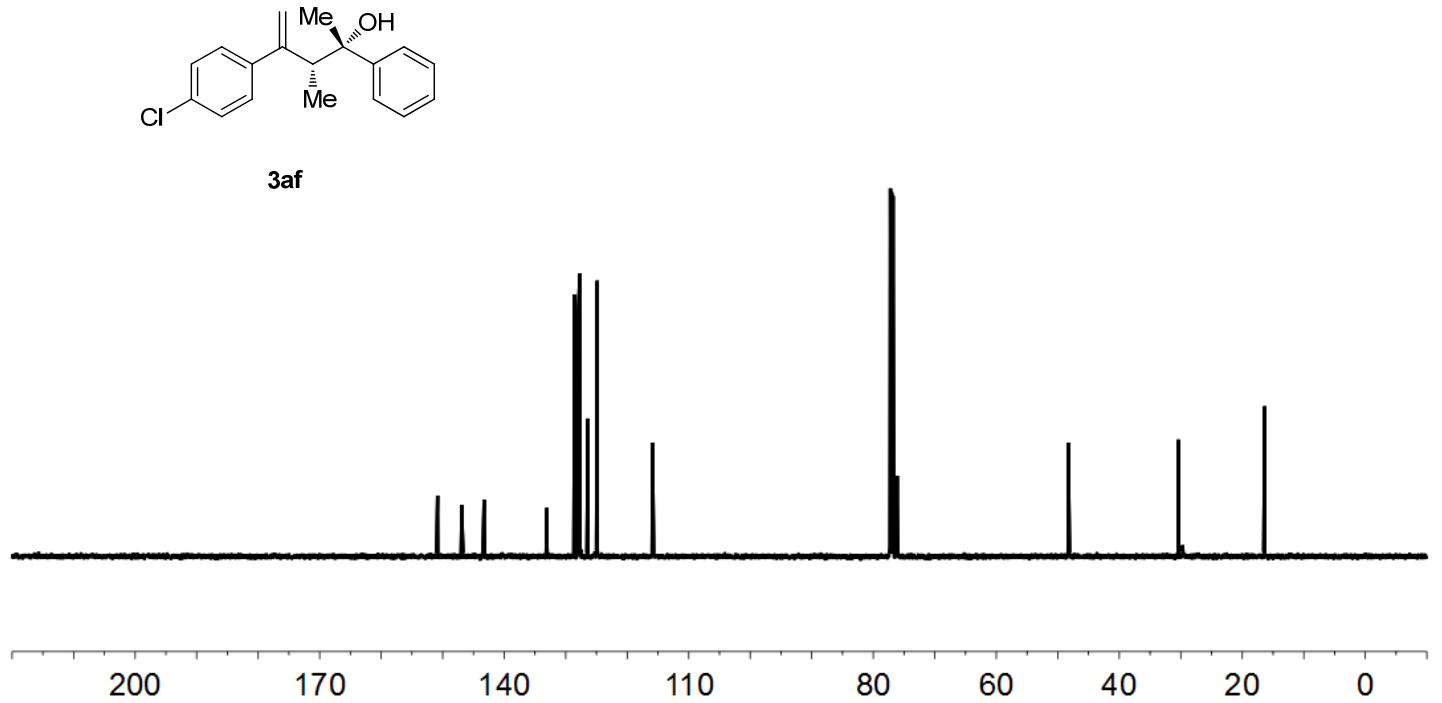



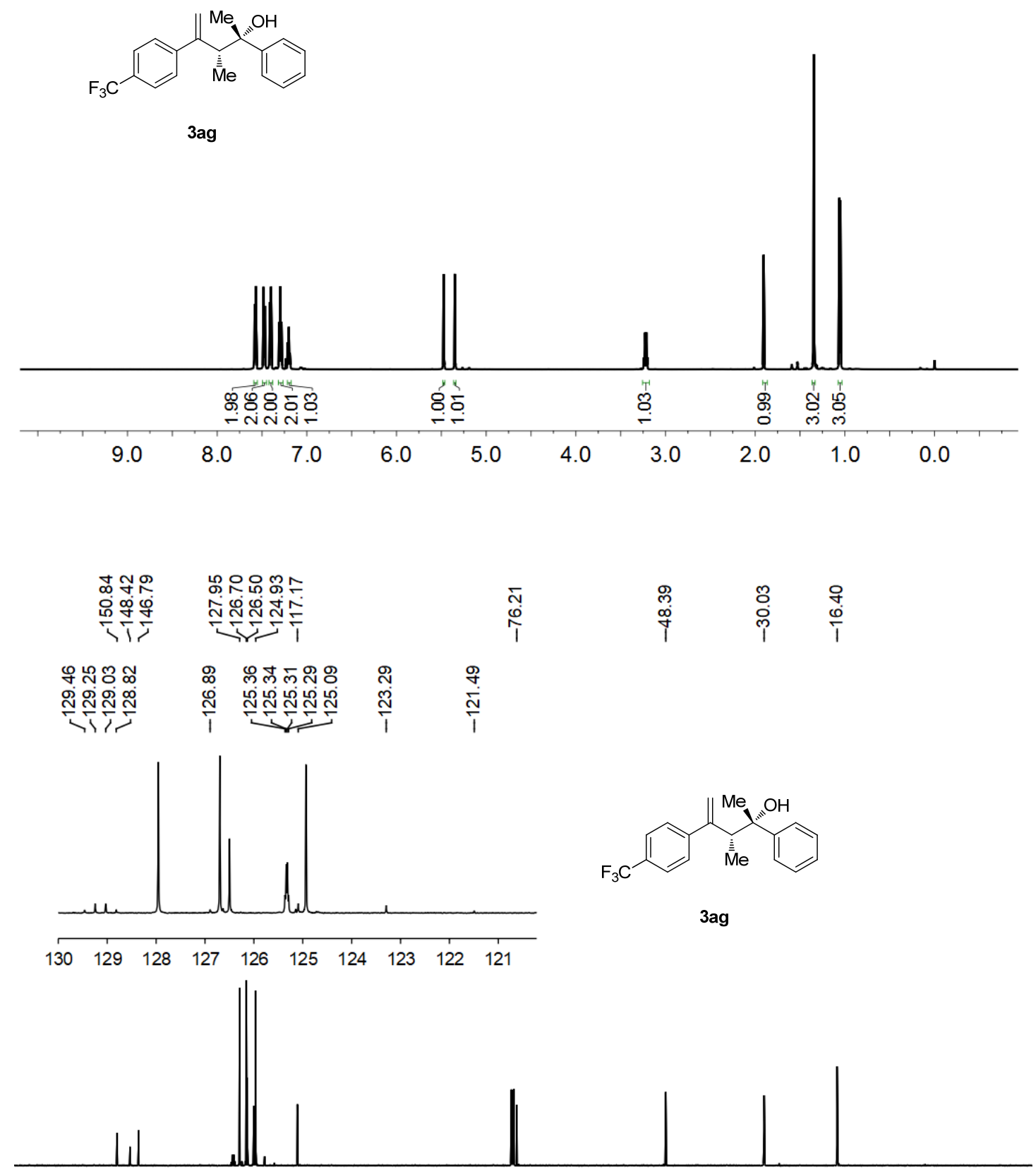

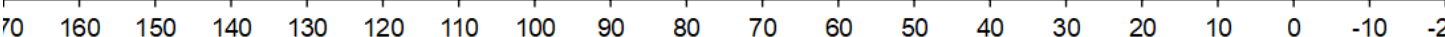




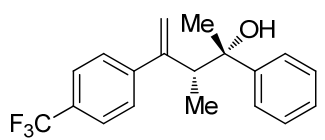

3ag

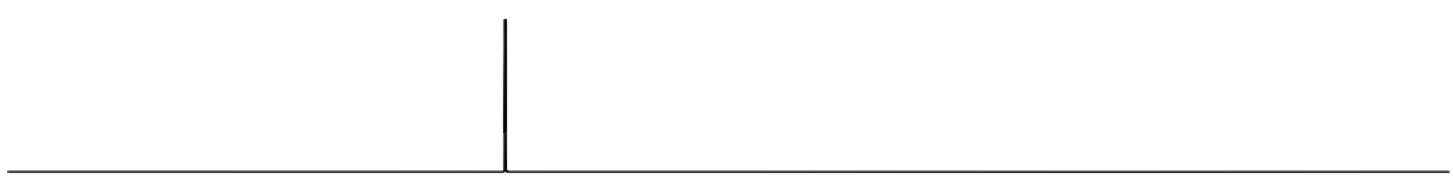

\begin{tabular}{llllllllllll}
\hline & 1 \\
\hline & 0 & -20 & -40 & -60 & -80 & -100 & -130 & -160 & -190 & -220 & 1
\end{tabular}



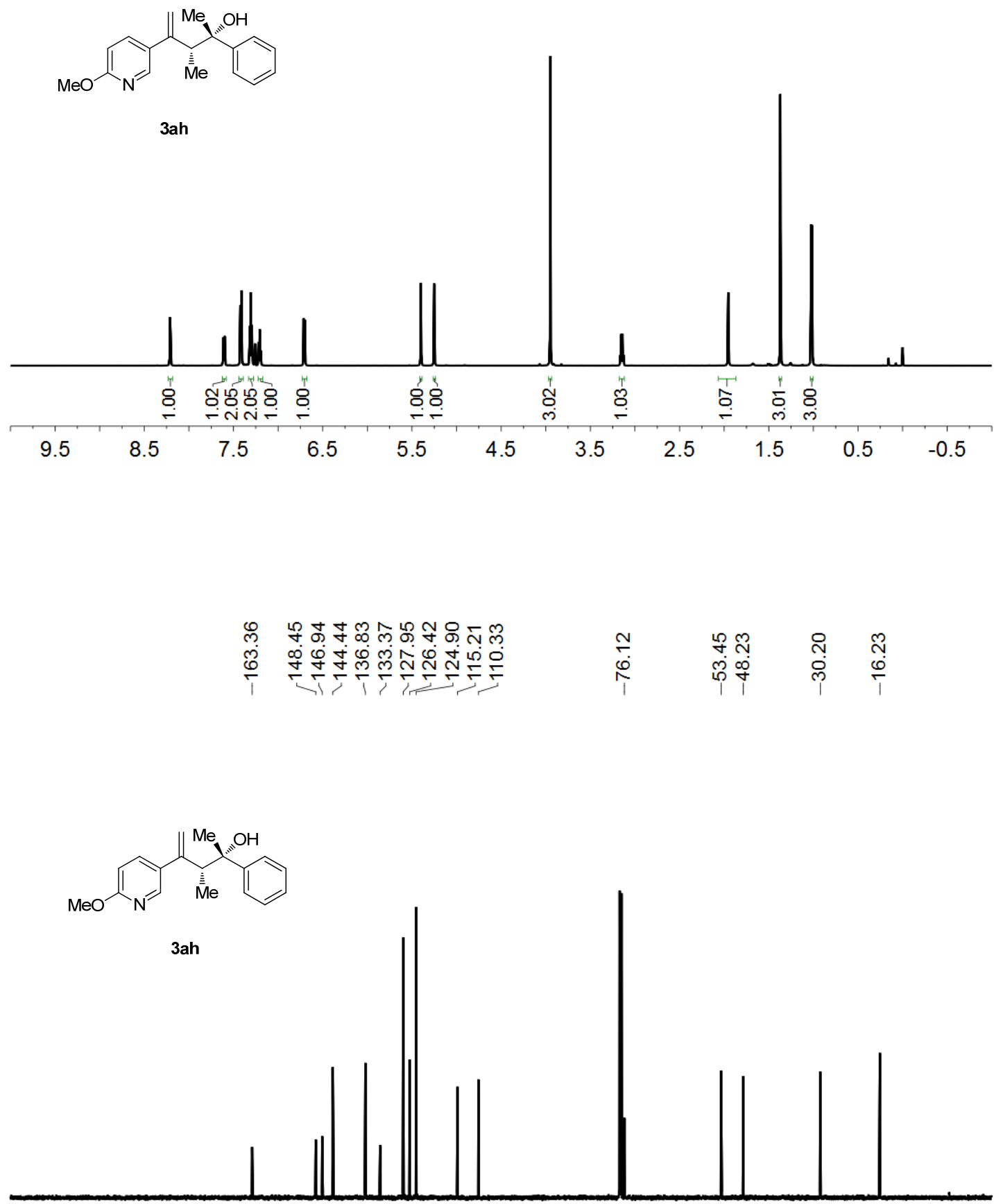


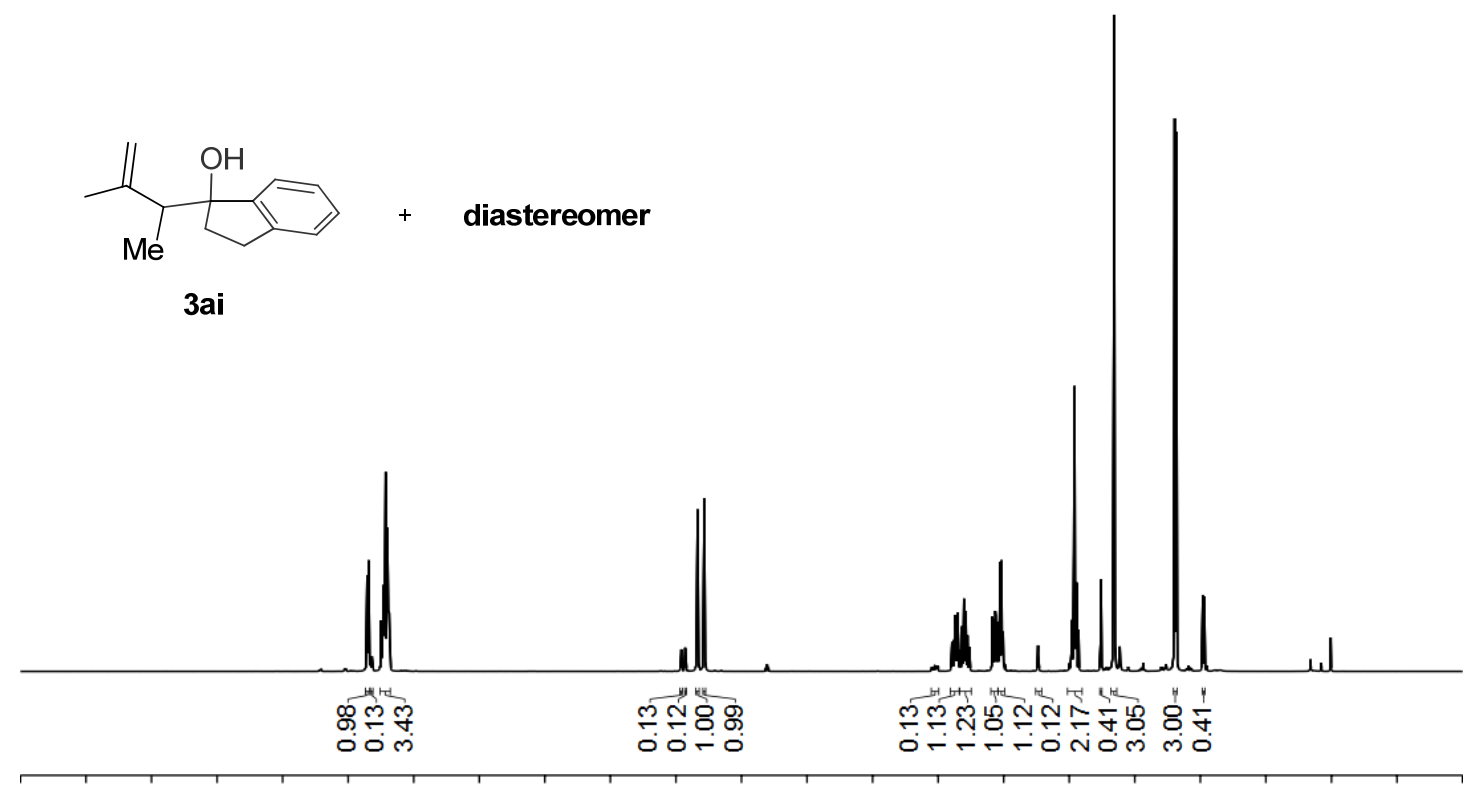

$\begin{array}{lllllllllllllllllllll}9.5 & 9.0 & 8.5 & 8.0 & 7.5 & 7.0 & 6.5 & 6.0 & 5.5 & 5.0 & 4.5 & 4.0 & 3.5 & 3.0 & 2.5 & 2.0 & 1.5 & 1.0 & 0.5 & 0.0 & -0.5\end{array}$

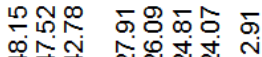

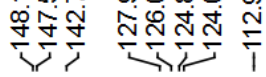

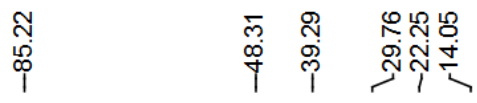

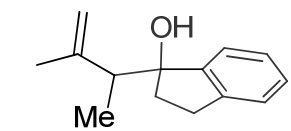

diastereomer

3ai

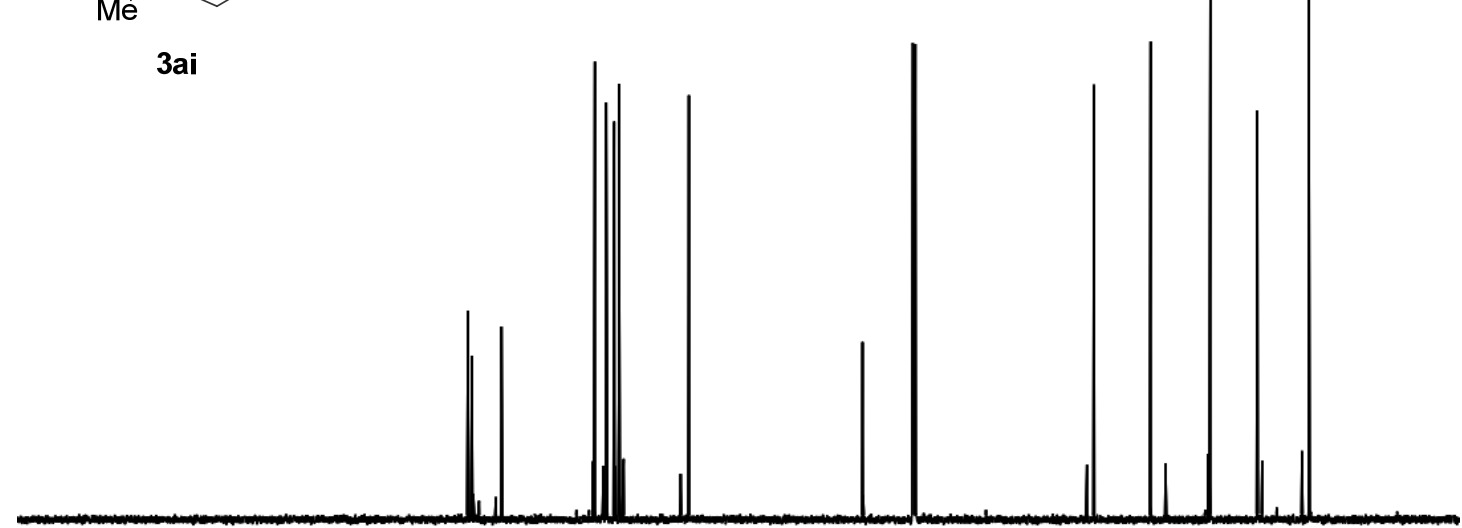

210

190

170

150

130

110

90

7060

$\begin{array}{llllll}40 & 30 & 20 & 10 & 0 & -1\end{array}$ 


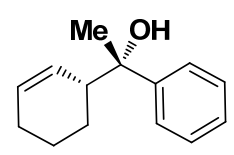

3aj

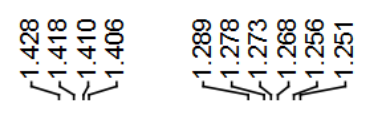

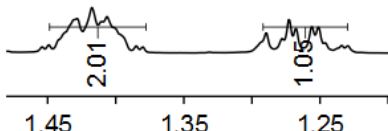

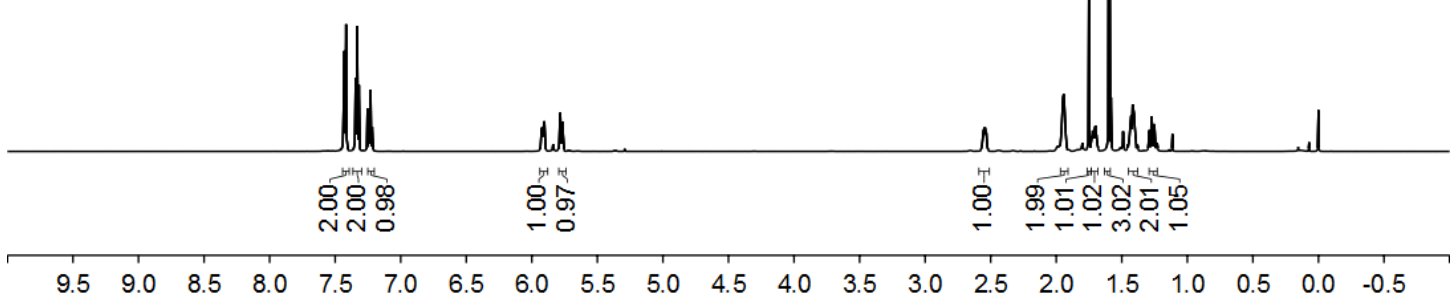

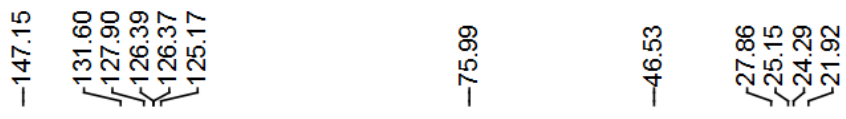

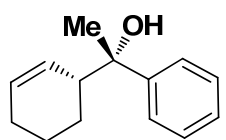

3aj

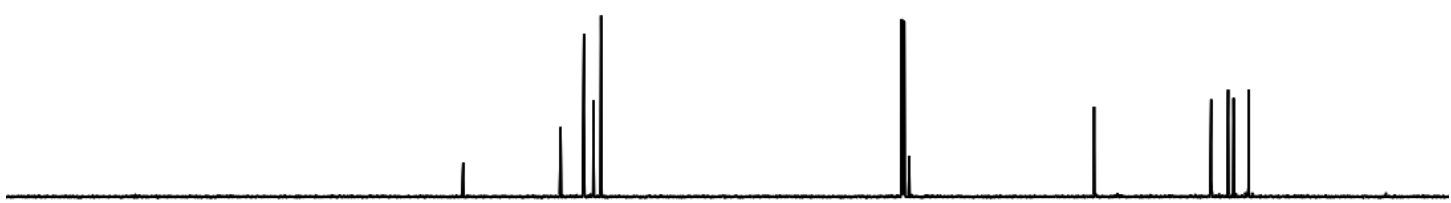

\begin{tabular}{|c|c|c|c|c|c|c|c|c|c|c|c|c|}
\hline 210 & 190 & 170 & 150 & 130 & 110 & $90 \quad 80$ & 70 & 60 & 50 & 40 & $3 c$ & \\
\hline
\end{tabular}




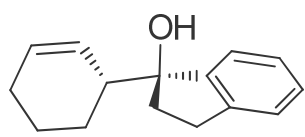

3ak

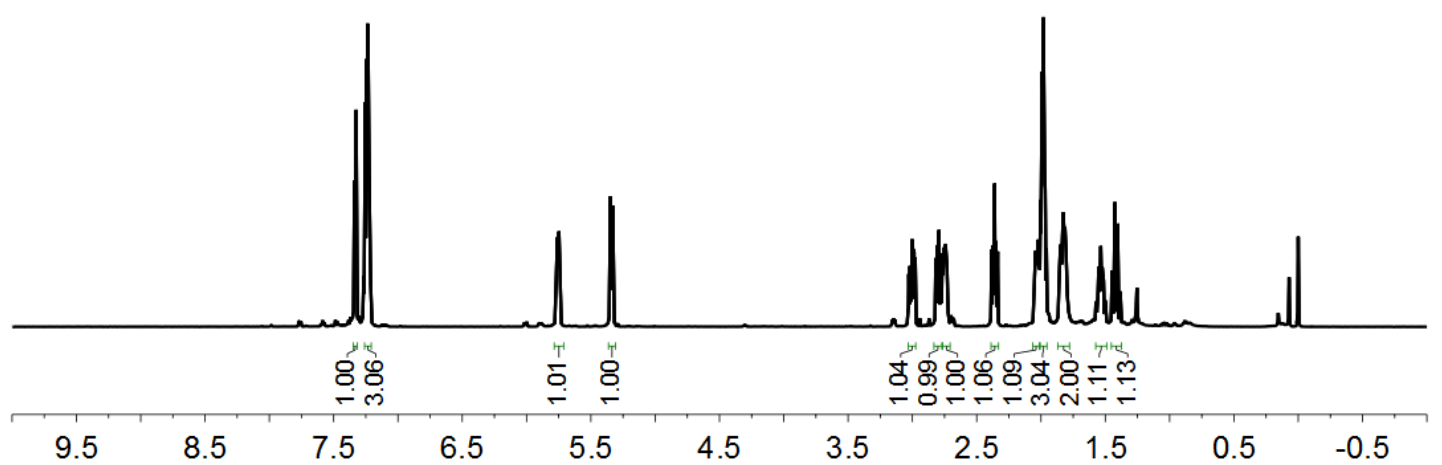

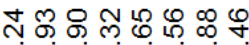

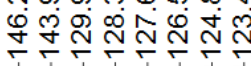
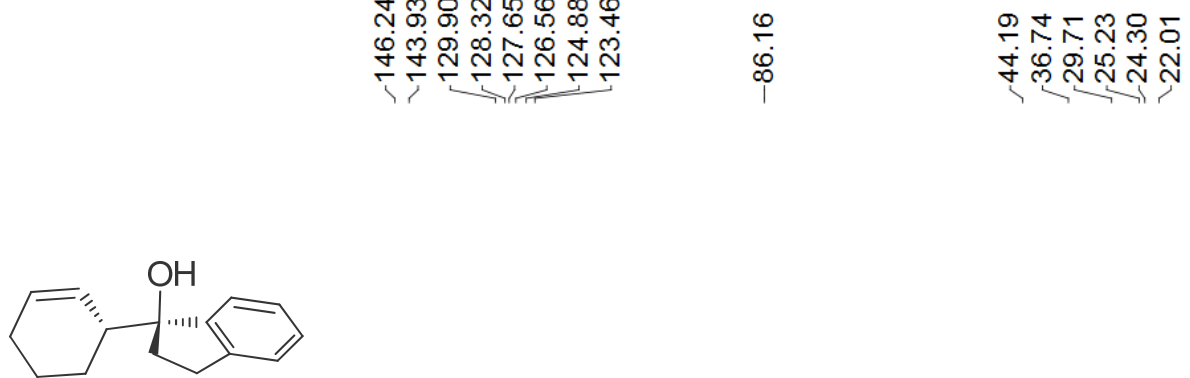

3ak

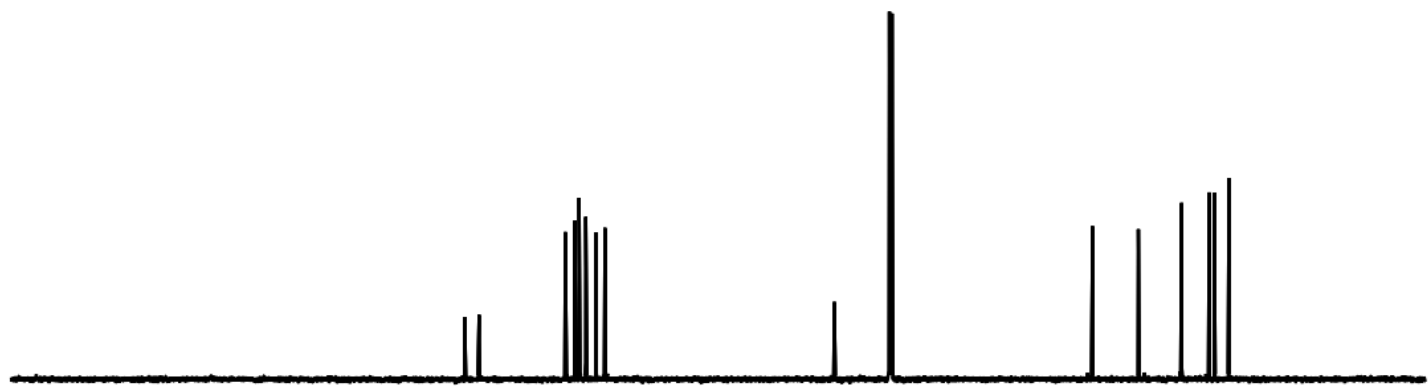

200

170

140

110

$80 \quad 60$

60

40

20

0 


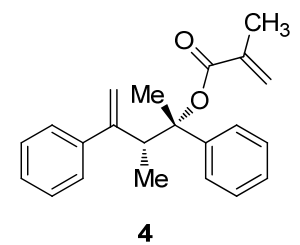

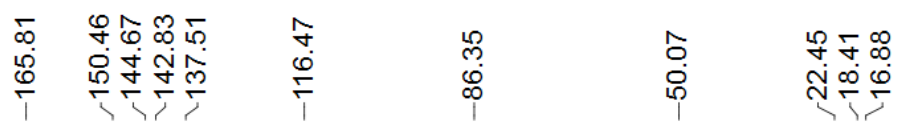
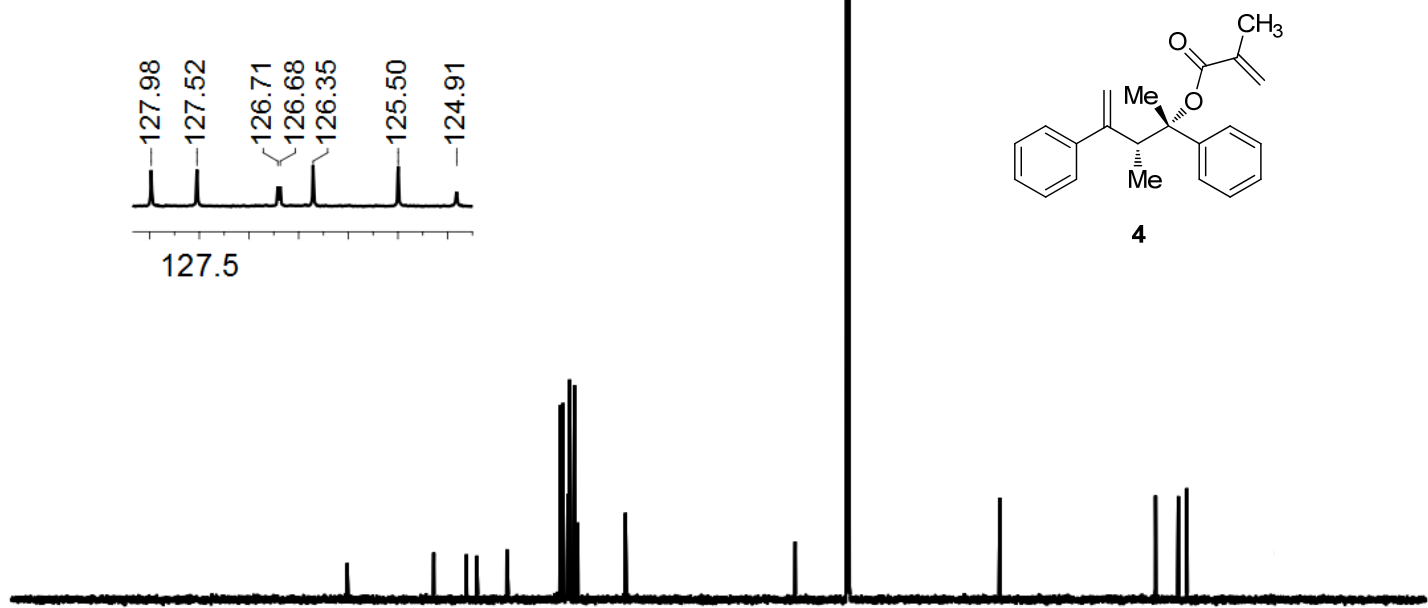

200

170

140

110

80

60

40

20

0 

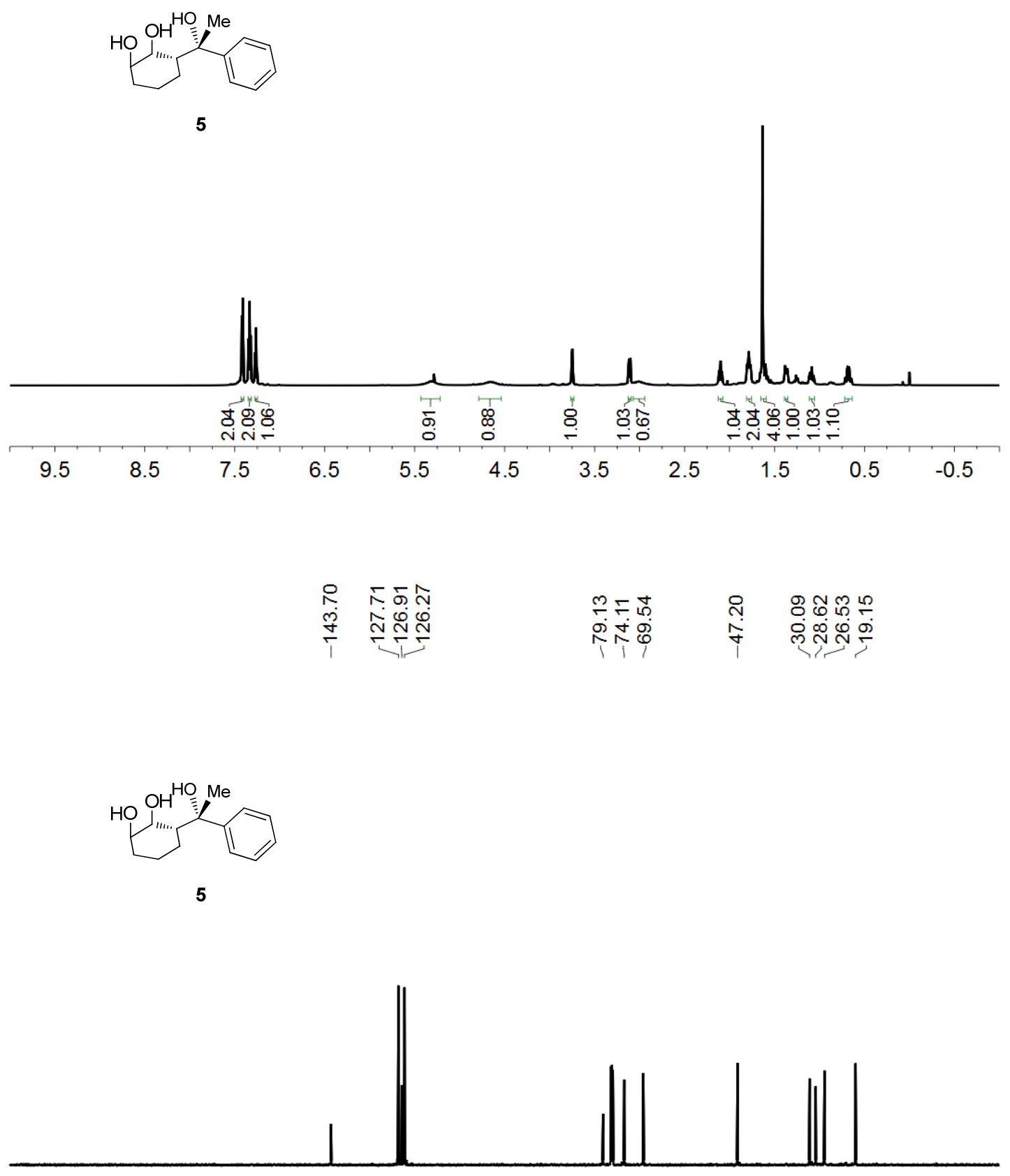

200

170

140

110

80

60

40

20

0 
